
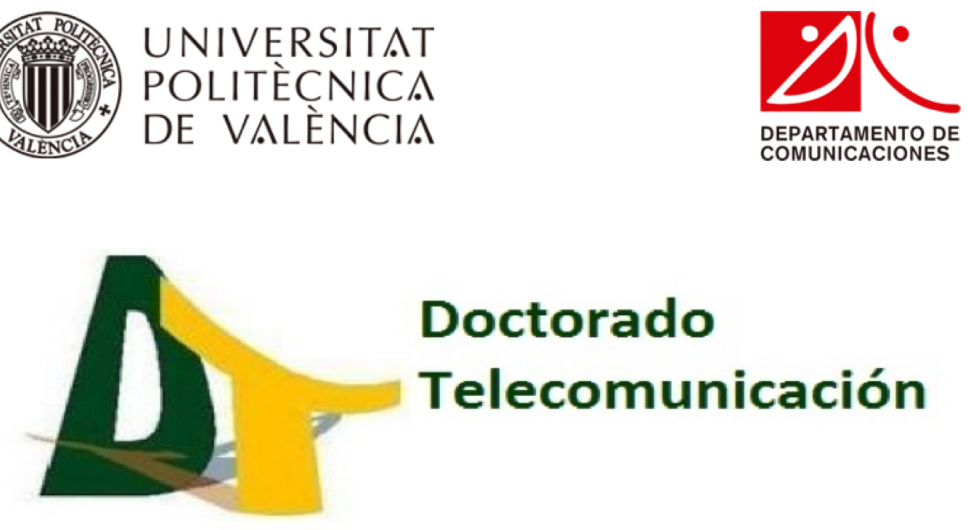

Doctorado

Telecomunicación

\title{
Development of direct measurement techniques for the in-situ internal alignment of accelerating structures
}

Author: $\quad$ Natalia Galindo Muñoz

$\begin{array}{ll}\text { Directors: } & \text { Dr. Ángeles Faus Golfe } \\ & \text { Dr. Vicente Enrique Boria Esbert }\end{array}$

Submission: February 2018

European Organization for Nuclear

Institutions: $\quad$ Research (CERN)

Instituto de Física Corpuscular (IFIC) 



\section{Acknowledgements}

In the first place, I would like to express my gratitude to the PACMAN project, Instituto de Física Corpuscular (IFIC) and Universitat Politècnica de València (UPV) for giving me the privileged opportunity to perform this work and PhD thesis. My special thanks go to the PACMAN leader, Dr. Hélène Mainaud-Durand, for her committement striving for the success of the project. My deep and eternal acknowledgements to my supervisor at CERN, Dr. Nuria Catalán Lasheras, for entrusting me with this responsibility, for her endless paciente and smart contributions in the development of this work; to my Ph.D. supervisors Dr. Ángeles Faus Golfe and Dr. Vicente Boria Esbert, for their dedication, involvement and bright professional and human guidance along these years.

I would like to seize this opportunity to to thank the valuable help I obtained from Anastasiya Solodko and Serge Lebet with the conceptual design of the test bench, mechanical designs and manufacturing. I am also very grateful to César Blanch Gutiérrez and Dr. Juan Jose Garcia Garrigós for their kind technical assistance at IFIC. I highlight the collaboration from Guy Deferne and Olaf Dunkel in the manufacturing of the crucial wirepositioning systems. I highly appreciate the many times Pablo Sobrino Monpean, Dr. Matteo Volpi and Philippe Guyard carried the heavy test bench from the radiofrequency to the metrology laboratory. Thanks to the team involved in the imperative metrology measurements and analysis: Dr. Ahmed Cherif, Didier Glaude, Mathieu Duquenne, Dominique Pugnat and Cyril Haerinck. I emphasize the valuable innovative ideas and fruitful discussions with Dr. Walter Wuensch, Manfred Wendt, Dr. Mika Dehler, Prof. Roger Jones, Dr. Erk Jensen, Dr. Gregoire Walkiers and specially Dr. Alexej Grudiev, who gently provided me all HFSS support. I assess the relevant trainings and wise advices on high-frequency VNA measurements from Dr. Andrea Mostacci, Andrej Olyudnin and Hikmet Bursali. Many thanks to Wilfrid Farabolini, Dr. Luis Navarro and Dr. Reidar Lunde Lillestol, for coaching my educational background on wakefield monitors. I would also like to name those who made possible the collaboration with National Instruments and my warm reception in Hungary: Botond Barabas, Peter Nemeti, Laszlo Abraham, Erika Kun, Joe Woodford, Agota Ivanyi, Elisabeth Barabas and Augusto Mandelli. My sincere thanks to Laurie Hemery for arranging all the administrative and travel formalities, always with kindness and efficiency. My special thanks to Jonhathan Pérez Blandino for the English revision. My gratitudes to Dr. Michele Modena, for organising all the professional and human productive dissemination and outreach activities within PACMAN. My great compliments to the competent PACMAN team and my acknowledgements for the wonderful experiences shared with: Claude Sanz, Domenico Caiazza, Solomon William Kamugasa, Vasileios Vlachakis, Giordana Severino, Peter Novotny, Silvia Zorzetti, Iordan Doytchinov and David Tshilumba. I would also like to address my gratitude to Dr. Olivier Brunner, for all his support along these years at CERN and letting me participate many interesting RF activities. I could not end before expressing my heartfelt gratitude to the special and unique friends I made at CERN: Dr. Marta Sabate Gilarte, Lorena Lobato Pardavila, Pablo Martínez Yanez, Gianfranco Ravida, Dr. Jorge Giner Navarro, Dr. Alfonso Benot Morell, Jorge Sanchez Quesada, Javier Galindo Guarch and Juan Carlos Alica.

No existen palabras suficientes que expresen mi inmenso agradecimiento hacia mis padres, Cristóbal y Antonia, y mi hermano Cristóbal, por el infinito apoyo recibido y confiar 
firmemente en el logro de mis objetivos. Je saisis cette occasion d'exprimer ma sincére et profonde gratitude à mon mari Maxime, pour son amour et ses encouragements qui ont permis la réalisation de cette travail. Je tiens à terminer en remerciant mes beaux parents, Brigitte et Cyril, pour le soutien et la confiance dont ils m'ont honoré. 


\section{Contents}

$\begin{array}{lr}\text { Summary } & 9\end{array}$

$\begin{array}{lr}\text { Resumen } & 12\end{array}$

$\begin{array}{lr}\text { Resum } & 13\end{array}$

1 Introduction to alignment in the next generation of particle accelerators 19

1.1 Introduction to the alignment of accelerator components . . . . . . . 19

1.2 Alignment in Future Linear Colliders (FLC): ILC and CLIC . . . . . . . 24

1.2.1 Beam-based alignment techniques in linear colliders . . . . . . 25

1.2.2 ILC versus CLIC alignment strategy . . . . . . . . . . . . . 28

1.3 The PACMAN project as a novel pre-alignment solution . . . . . . . 30

1.3.1 The pre-alignment strategy of PACMAN . . . . . . . . . 31

1.3.2 Scientific Program of PACMAN . . . . . . . . . . . . . 32

1.3.3 The innovative aspects coming along the success of the program . 36

2 New methodology and EM field simulations to measure the EM axis of Accelerating Structures: the case of the CLIC TD24 39

2.1 The TD24 AS developed for CLIC . . . . . . . . . . . . . . . . . 39

2.2 Current technique to estimate the geometric centre of AS . . . . . . . . 42

2.3 New methodology to measure the EM centre of AS for alignment purposes 44

2.3.1 Wire as an excitation: the coaxial wire method and the twinax

method ...................... 44

2.3.2 The new technique: the perturbative method . . . . . . . . 52

2.4 Experimental proposal to measure the EM axes of the CLIC TD24 AS . . 57

2.5 EM field simulation studies to find the EM axes of the CLIC TD24 AS

with the PACMAN wire . . . . . . . . . . . . . 58

2.5.1 EM fields and $S$ parameters in the middle cell with and without a

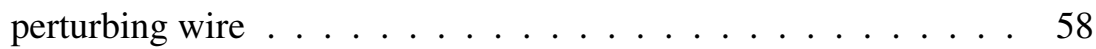

2.5.2 Accuracy and resolution study . . . . . . . . . . . . . 68

2.5.3 Characterisation of errors . . . . . . . . . . . . . . 68

2.5.4 Impact study of the material and wire radius . . . . . . . . 76

3 Design, fabrication and calibration of an experimental set-up to measure the EM axes of the CLIC TD24 using the perturbative method 79

3.1 Design of the test-bench . . . . . . . . . . . . . . . . . 79

3.2 Calibration of the experimental set-up and error estimation . . . . . . . 82

3.2.1 Calibration of the linear stages . . . . . . . . . . . . 84 
3.2.2 Calibration of the wire tilt $\ldots \ldots \ldots \ldots \ldots$. . . . . . 94

3.2 .3 Calibration of the VNA . . . . . . . . . . . . . . . . 96

3.3 Automatisation of the measurements . . . . . . . . . . . . . 97

3.3.1 Conceptual design of the algorithm . . . . . . . . . . . . 97

3.3.2 Software development for the automated algorithm performance . 100

3.4 Further developments of the measurement of the EM centre of AS . . . . 106

3.4.1 The cell-to-cell misalignment measurement . . . . . . . . . . . 106

3.4.2 The measurement of the EM axes simultaneously in various cells 107

3.4.3 Development of a test bench to measure the EM axis of the TD24 parallel to the beam direction . . . . . . . . . . . . . . . 111

4 Experimental measurements of the EM axes of the TD24 and analysis using the perturbative method 115

4.1 Experimental proof-of-principle with WFM signals . . . . . . . . . . . 115

4.2 Experimental measurements of the EM axes with tapered transitions . . . 123

4.3 Algorithm optimisation and final results . . . . . . . . . . . . . . . 125

4.3.1 Repeatability analysis of the perturbative method with metrology measurements . . . . . . . . . . . . . 132

4.4 Experimental identification of possible sources of errors . . . . . . . . . 134

4.4.1 Impact of rotating the TD24 around the longitudinal axis in the measurement of the EM centre . . . . . . . . . . . . . . 134

4.4.2 Impact of the coupling between the tapered transitions and the middle cell in the measurement of the EM centre . . . . . . . . 137

4.4.3 Influence of temperature in the measurement of the EM axes . . . 137 


\section{Summary}

In the next generation of linear particle accelerators, challenging alignment tolerances are required in the positioning of the components focusing, accelerating and detecting the beam over the accelerator length in order to achieve the maximum machine performance. In the case of the Compact Linear Collider (CLIC), accelerating structures, beam position monitors and quadrupole magnets need to be aligned in their support with respect to their reference axes with an accuracy of $10 \mu \mathrm{m}$. To reach such objective, the PACMAN (Particle Accelerator Components Metrology and Alignment to the Nanometer Scale) project strives for the improvement of the current alignment accuracy by developing new methods and tools, whose feasibility should be validated using the major CLIC components.

This Ph.D. thesis concerns the investigation, development and implementation of a new non-destructive intracavity technique, referenced here as the perturbative method, to determine the electromagnetic axes of accelerating structures by means of a stretched wire, acting as a reference of alignment. Of particular importance is the experimental validation of the method through the $5.5 \mathrm{~mm}$ iris-mean aperture CLIC prototype known as TD24, with complex mechanical features and difficult accessibility, in a dedicated test bench.

In the first chapter of this thesis, the alignment techniques in particle accelerators and the novel proposals to be implemented in the future linear colliders are introduced, and a detailed description of the PACMAN project is provided. The feasibility study of the method, carried out with extensive electromagnetic fields simulations, is described in chapter 2, giving as a result, the knowledge of the theoretical accuracy expected in the measurement of the electromagnetic axes and facilitating the development of a measurement algorithm. The conceptual design, manufacturing and calibration of the automated experimental set-up, integrating the solution developed to measure the electromagnetic axes of the TD24, are covered in chapter 3. The future lines of research and developments of the perturbative method are also explored. In chapter 4 , the most significant results obtained from an extensive experimental work are presented, analysed and compared with simulations. The proof-of-principle is completed, the measurement algorithm is optimised and the electromagnetic centre is measured in the TD24 with a precision less than $1 \mu \mathrm{m}$ and an estimated error less than $\pm 8.5 \mu \mathrm{m}$. Finally, in chapter 5, the developments undertaken along this research work are summarised, the innovative achievements accomplished within the PACMAN project are listed and its impact is analysed. 


\section{Resumen}

Las exigentes tolerancias de alineación en los componentes de los futuros colisionadores lineales de partículas requieren el desarrollo de nuevas técnicas de alineación más precisas que las existentes. Este es el caso del Colisionador Lineal Compacto (Compact Linear Collider, CLIC), cuyos objetivos altamente restrictivos de alineamiento alcanzan los $10 \mu \mathrm{m}$. Para poder lograr el máximo rendimiento del acelerador, es necesario que el posicionamiento de las estructuras que aceleran las partículas y de los campos que las guían cumplan las tolerancias de alineación para dirigir el haz a lo largo de la trayectoria diseñada. Dicho procedimiento consiste en relacionar la posición de los ejes de referencia de cada componente con respecto a objetos externos, o fiduciales, lo cual resulta muy tedioso y económicamente costoso. Los errores sistemáticos y aleatorios se van acumulando en cada paso del proceso y, en consecuencia, la precisión final de alineamiento es todo un desafío.

En este contexto, nace el proyecto PACMAN (Particle Accelerator Components Metrology and Alignment to the Nanometre scale), subvencionado por la Unión Europea en el programa FP7 de financiación para la investigacíon e innovación. El objetivo principal de PACMAN es investigar, desarrollar e implementar una solución integrada alternativa que incorpore todos los pasos de alineación en una misma ubicación, con el objetivo de mejorar la precisión de alineación de los componentes de los aceleradores, en concreto: las estructuras aceleradoras, los cuadrupolos y los monitores de posición de haz. La viabilidad de las soluciones desarrolladas y la precisión de alineamiento alcanzada deben de demostrarse en un banco de pruebas utilizando componentes de CLIC. La estrategia de PACMAN para alcanzar el objetivo técnico se divide en tres pasos. El primero consiste en la fiducialización de los componentes y sus soportes. El segundo paso es el ensamblaje de los componentes en dos tipos de soporte, uno compuesto por un monitor de posición de haz y un cuadrupolo, y otro con cuatro estructuras aceleradoras, tomando como referencia su centro electromagnético. Finalmente, ambos soportes se transportan al túnel para su alineación final utilizando técnicas de hilos tensados.

En esta tesis doctoral, se describe el desarrollo de una nueva técnica no destructiva para localizar los ejes electromagnéticos de estructuras aceleradoras y su validación experimental. Para ello, se ha utilizado una estructura aceleradora de CLIC conocida como TD24. En el primer capítulo, se realiza una introducción a las técnicas de alineamiento en los aceleradores de partículas y a las novedosas propuestas de futuros colisionadores lineales. Finalmente, se describe en detalle el proyecto PACMAN. 
En el segundo capítulo, se presentan las características técnicas de la TD24 y se realiza una revisión del estado del arte de los métodos de medidas del centro electromagnético de estructuras aceleradoras. Debido a la complejidad mecánica de la TD24, su difícil acceso y su diámetro medio de iris de $5.5 \mathrm{~mm}$, se desarrolla una nueva técnica denominada en esta tesis como el método perturbativo y se realiza una propuesta experimental de validación. El estudio de viabilidad de este método, cumpliendo con los requisitos impuestos de precisión en la medida de $10 \mu \mathrm{m}$, ha sido realizado con una campaña extensa de simulaciones de campos electromagnéticos en tres dimensiones utilizando la herramienta de software conocida como HFSS. Los resultados de simulación han permitido el desarrollo de un algoritmo muy completo de medidas y han proporcionado las especificaciones técnicas para el diseño conceptual de un banco de pruebas para la medida de los ejes electromagnéticos de la TD24.

En el capítulo tres, se describe el diseño, la fabricación y la calibración del banco experimental. Este proceso ha tenido en consideración una configuración sencilla de sus elementos para mover la estructura aceleradora en dos grados de libertad alrededor del hilo y localizar los ejes electromagnéticos en un plano ortogonal, además de facilitar la inserción del hilo a lo largo de la estructura sin dañar su superficie interna. Para poder obtener la precisión deseada en la medida de los ejes con el mínimo error, en el montaje del banco se emplea el instrumento de medida de coordenadas (Coordinate Measuring Machine, CMM) más novedoso en el CERN, con solo $0.3 \mu \mathrm{m}$ de incertidumbre. Asimismo, se ha desarrollado e integrado en LabVIEW una plataforma de usuario para la realización automática del algoritmo de medición de los ejes. Además, se proponen futuras líneas de investigación y desarrollo del método perturbativo, cuya viabilidad han sido previamente examinadas.

En el capítulo 4, se resumen los numerosos test experimentales realizados con el banco, cuyos resultados se analizan y comparan con los obtenidos mediante simulación. En una primera etapa, se realiza la prueba de principio de los supuestos del método y la primera medición del centro electromagnético con el banco, aún en fase inicial de desarrollo. A continuación, se procede con las calibraciones de los instrumentos de medida y se reemplazan elementos diseñados y elaborados para mejorar la precisión. La finalización del banco de pruebas y sus correspondientes calibraciones, la incorporación de nuevos tratamientos de las medidas en el algoritmo final y la caracterización de fuentes de error en la medida, favorecieron la localización del centro electromagnético en la TD24 con una precisión menor a $1 \mu \mathrm{m}$ con un error estimado menor que $\pm 8.5 \mu \mathrm{m}$, cumplimiendo con los objetivos de precisión establecidos.

Finalmente, en el capítulo 5, se realiza un resumen del trabajo de investigación desarrollado a lo largo de esta tesis. Asimismo, se enumeran y analizan los logros innovadores efectuados en el proyecto PACMAN y su impacto. 


\section{Resum}

En la generació próxima d'acceleradors de partícules lineals, desafianttoleràncies d'alineament és requerit en el posicionament dels components que enfoquen, accelerant i detectant la biga sobre la longitud d'accelerador per tal d'aconseguir l'actuació de màquina màxima. En el cas del Colisionador Compacte Lineal (CLIC), accelerant estructures, monitors de posició de fes i imants necessiten ser alineats en el seu suport amb respectar a les seves destrals de referència amb una precisió de $10 \mu \mathrm{m}$. Per assolir tal objectiu, elprojecte PACMAN (Metrologia de Components de l'Accelerador de partícules iAlineament alNanometer Escala)s'esforer la millora de l'actual precisió d'alineament per mètodes nous en desenvolupament $\mathrm{i}$ eines, la viabilitat dels quals hauria de ser validada utilitzant els components de CLIC importants.

Aquesta tesi concerneix la investigació, desenvolupament i implementació d'un nou nodestructiu tècnica interna, va referenciar ací mentre el mètode de pertorbació per determinar les destrals electromagnètiques d'accelerar estructures mitjant un cable estès, actuant com a referència d'alineament. De la importància particular és la validació experimental del mètode a través del $5.5 \mathrm{~mm}$ iris-roíobertura prototipus de CLIC sabut com TD24, amb característiques mecàniques complexes i accessibilitat difícil, en un banc de prova dedicat.

En el primer capítol d'aquesta tesi, les tècniques d'alineament en acceleradors de partícules i les propostes novelles per ser implementades en el futur colisionador lineal ntroduit, i una descripció detallada delprojecte PACMAN és proporcionat. L'estudi de viabilitat el mètode de pertorbació, va dur a terme amb simulacres de camps electromagnètics extensos, és descrit dins capitol 2, donant com a resultat, el coneixement de la precisió teórica esperada en la mida de les destrals electromagnètiques i facilitant el desenvolupament d'un algoritme de mida. El disseny conceptual, fabricació icalibratge del conjunt experimental automatitzat-amunt, integrant la solució desenvolupada per mesurar les destrals electromagnètiques del TD24, és cobert dins capitol 3. Les línies futures de recerca i desenvolupaments del mètode és també va explorar. Dins capítol 4, la majoria de resultats significatius van obtenir d'una faena experimental extensa és presentada, analitzat i comparat amb simulacres. La prova-de-el principi és completat, l'algoritme de mida és optimitzat i el centre electromagnètic és mesurat en el TD24 amb una precisió menys d'1 um i un error calculat menys de $\pm 8.5 \mu \mathrm{m}$. Finalment, dins capítol 5, els desenvolupaments empresos al llarg d'aquesta faena de recerca ésresumit, les consecucions innovadores van acomplir dins delprojecte PACMAN és llistat i el seu impacte és analitzat. 


\section{List of Acronyms}

AFC Alignment and Fiducialisation of each Component

AS Accelerating Structure

ATF Accelerator Test Facility

BBA Beam Based Alignment

BPM Beam Position Monitor

CATIA Computer-Aided Three Dimensional Interactive Application

CDR Conceptual Design Report

CesrTA Cornell Electron-Positron Storage Ring Test Accelerator

CLIC Compact Linear Collider

CMM Coordinate Measuring Machine

CTF2 CLIC Test Facility 2

CTF3 CLIC Test Facility 3

DFS Dispersion-Free Steering

DMS Dispersion-Matched Steering

DS2 Detuned Structure number 2

EDMS Equipment Data Management Service

EM Electro Magnetic

ESR Early Stage Researchers

FLC Future Linear Colliders 
FPAB Final PACMAN Alignment Bench

FSI Frequency Scanning Interferometry

GPS Global Positioning System

HEP High Energy Physics

IFCA International Committee for Future Accelerators

IFIC Instituto de Fisica Corpuscular

ILC International Linear Collider

ITN Innovative Training Network

HOM Higher Order Mode

KEK High Energy Accelerator Research Organisation

KM Kick Minimization

LabVIEW Laboratory Virtual Instrument Engineering Workbench

LCC Linear Collider Collaboration

LHC Large Hadron Collider

MRN Metrological Reference Network

NI National Instruments

NLC Next Linear Collider

PACMAN Particle Accelerator Components Metrology and Alignment to the Nanometre scale

PCB Printed Circuit Board

PETS Power Extraction and Transfer Structure

PXI PCI eXtensions for Instrumentation

SESHAT Shape Evaluating Sensor: High Accuracy and Touchless

SLAC Stanford Linear Accelerator Centre 
SMA SubMiniature version A

SPN Support Pre-alignment Network

TDR Technical Design Report

TT1 Transfer Tunnel 1

VI Virtual Instruments

VNA Vector Network Analyzer

WFM Wakefield Monitors

WP Workpackage

WPS Wire Positioning Sensors 


\section{Chapter 1}

\section{Introduction to alignment in the next generation of particle accelerators}

The recent discovery of the Higgs boson at the Large Hadron Collider (LHC), confirming the Standard Model at energy levels never explored before, have caused an important progress in the field of particle physics and have launched a new era of research in High Energy Physics (HEP). The LHC is currently the most powerful accelerator in the world and the largest scientific instrument ever built. Its full exploitation is one of the highest priorities in the field, but the LHC program will cease at some time around 2035-2040 [1]. In view of this, the feasibility of particle colliders with even higher energy and/or higher precision with very demanding parameters and performance are being investigated by the particle and accelerator physics community.

One of the challenges the construction of a particle accelerator is facing, lies in the translation of the theoretical layout, designed in Cartesian space, into a physical accelerator in geocentric space, which is defined as the alignment. The positioning of the accelerator components supplying guide fields and accelerating forces to the particles, like magnets and Accelerating Structures (AS), needs to score the alignment tolerances to steer the beam along the designed trajectory to achieve the maximum performance of the machine.

In this chapter, some of the general alignment methods used for accelerator components are introduced. Techniques and technology to improve alignment accuracy and precision for the next generation of particle accelerators are discussed. Finally, the innovative research programme, which gave the frame of this Ph.D. thesis, is presented.

\subsection{Introduction to the alignment of accelerator components}

A six-step alignment procedure is followed to position each of the accelerator components, specified in local coordinate system, into the foreseen location of the accelerator tunnel which uses a common coordinate system defined by the nominal beam trajec- 


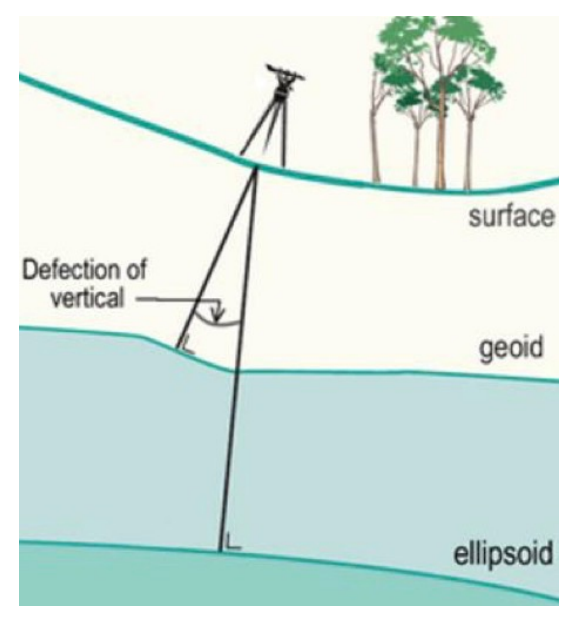

Figure 1.1: Model of the Earth and datum required to determine the survey reference frame [3].

tory [2] [3].

In the first step a survey reference frame is defined, which is basically a survey coordinate system appropriate to the project site and size. This reference frame is established in form of a mathematical model of the survey space, in which the surveyor takes measurements and analyzes the data. For large projects, like the LHC with $27 \mathrm{~km}$ circumference, the non-perfect spherical shape of the earth makes this definition a challenging task by its own. Therefore, the earth is modelled using two surfaces, called the horizontal and vertical geodetic datums, as illustrated in Figure 1.1. Evidently, the geodetic issues vary for different accelerator sizes. The geoid is defined as the gravitational equipotential surface, represented by the mean sea level, normal to the gravity vector. Consequently, it is the reference surface, and used as vertical geodetic datum. The equipotential of the gravity is determined at the surface level, by zenital camera measurements together with gravimetric measurements. For the horizontal geodetic datum an ellipsoid is defined for large accelerators, which approximates the shape of the earth for all survey measurements. The deflection of vertical is defined as the angle between the gravity vector normal to the geoid - and the plane normal to the ellipsoid. Different methods exist to measure the deflection of vertical in order to set up the geodesic reference network in an accelerator tunnel, similar to well-known astronomical methods. Due to the complexity of the ellipsoidal coordinates in analytical computations, the spheroidal coordinates are projected into a local Cartesian coordinate system, and combined with astro-geodetic measurements. Finally, all the distortions within the survey reference frame are mathematically modelled and corrected.

Beside the definition of the survey space, this first step also defines a survey network. The principle of a surface network is based on the installation of a set of pillar-type columns, in close proximity to each accelerator tunnel access pit, as illustrated in Figure 1.2. In the past, the coordinates of these pillars were measured on the surface using triangulation methods. Now, this is replaced by satellite-based measurement methods, like the Global Positioning System (GPS), achieving accuracies of approximately $1 \mathrm{~mm}$. The alignment network down in the accelerator tunnel is linked to the alignment network on the surface 


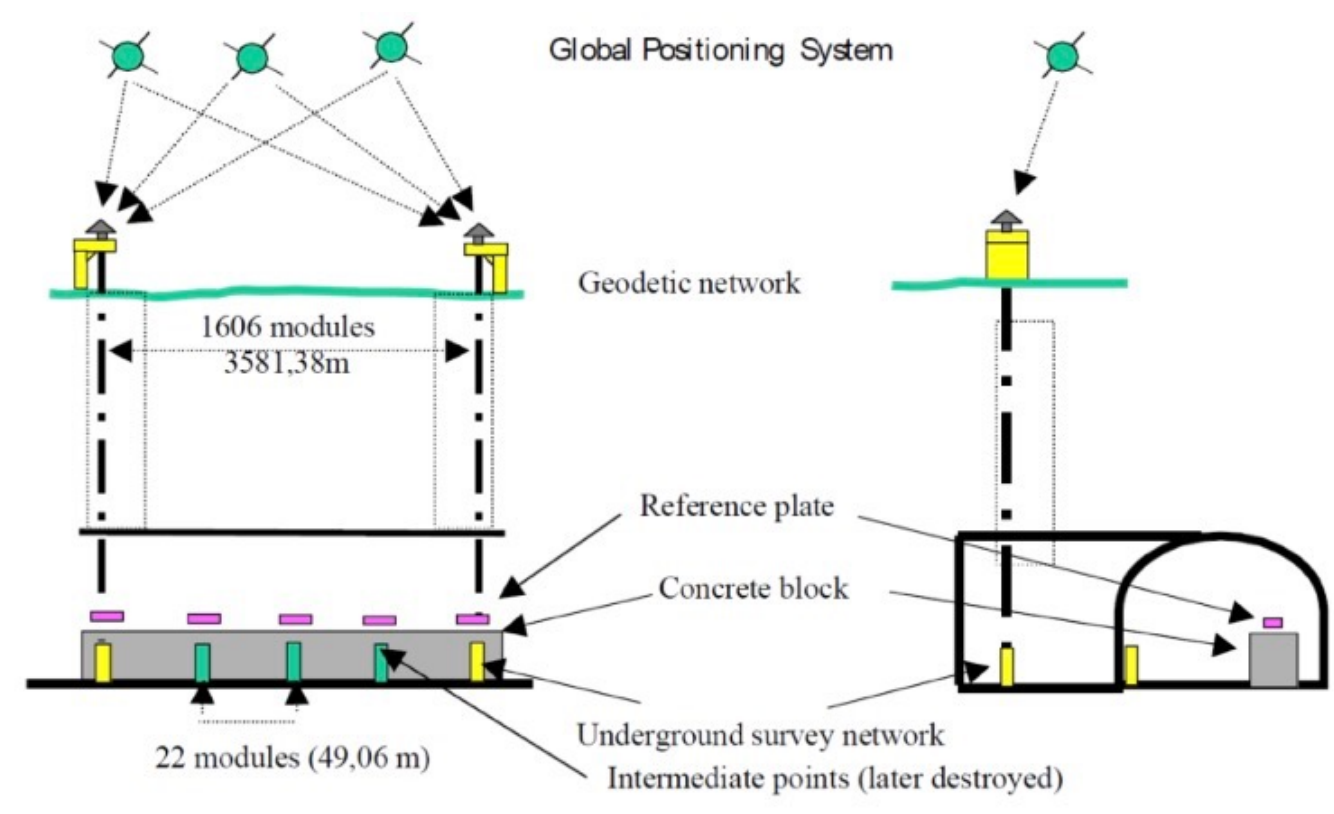

Figure 1.2: Installation and determination of the survey network. Courtesy of [3].

network about every $5 \mathrm{~km}$, scaled to $500 \mathrm{~m}$ in the tunnel, using a vertical drop procedure by optically or mechanically centring a tripod-mounted translation stage on the surface over a pillar column in the tunnel through a survey shaft. The tunnel networks are defined as longitudinal alignment networks and include pillar columns in the walls and on the ground, as illustrated in Figure 1.3. The position of these pillars can also be measured by using laser trackers or high precision tachometers, like the Leica TDA500, which can be operated in free-stationing mode. This opto-electronic instrument is equipped with an electronic distance meter and angular encoders, enabling continuous measurements of a spheric target, thanks to a target recognition software and motorised controls. Typically, these systems achieve a relative measurement accuracy of $100 \mu \mathrm{m}$ to locate the alignment sphere on the pillar column, while their absolute accuracy is directly correlated to the position accuracy of the surface network.

The second task to be carried out lies in the description of the layout of the reference frame, where the beam line is located. This layout description, and the specifications of all the components of the accelerator is defined by the lattice and the individual technical designs, including the tolerances of their installation position, i.e. their maximum allowed displacements. This parameter distinguishes between absolute and relative positioning. The absolute positioning tolerance specifies how close an individual component is from its theoretical position, while the relative positioning tolerance specifies the alignment quality of many components. For example, the absolute accuracy value is typically 3 to $4 \mathrm{~mm}$, required along a $3 \mathrm{~km}$ long sector of an accelerator in the case of the LHC with a relative accuracy between adjacent alignment monuments of $0.15 \mathrm{~mm}$ rms $(1 \sigma)$. Redundancy on the observation is necessary to assure that the surveying measurement show a typical Gaussian distribution with $1 \sigma$ confidence level. All the coordinate transformations need to be specified in this step in order to be related with the first task, so a transformation matrix defines the relationship between the surveying and lattice coordinate systems. 


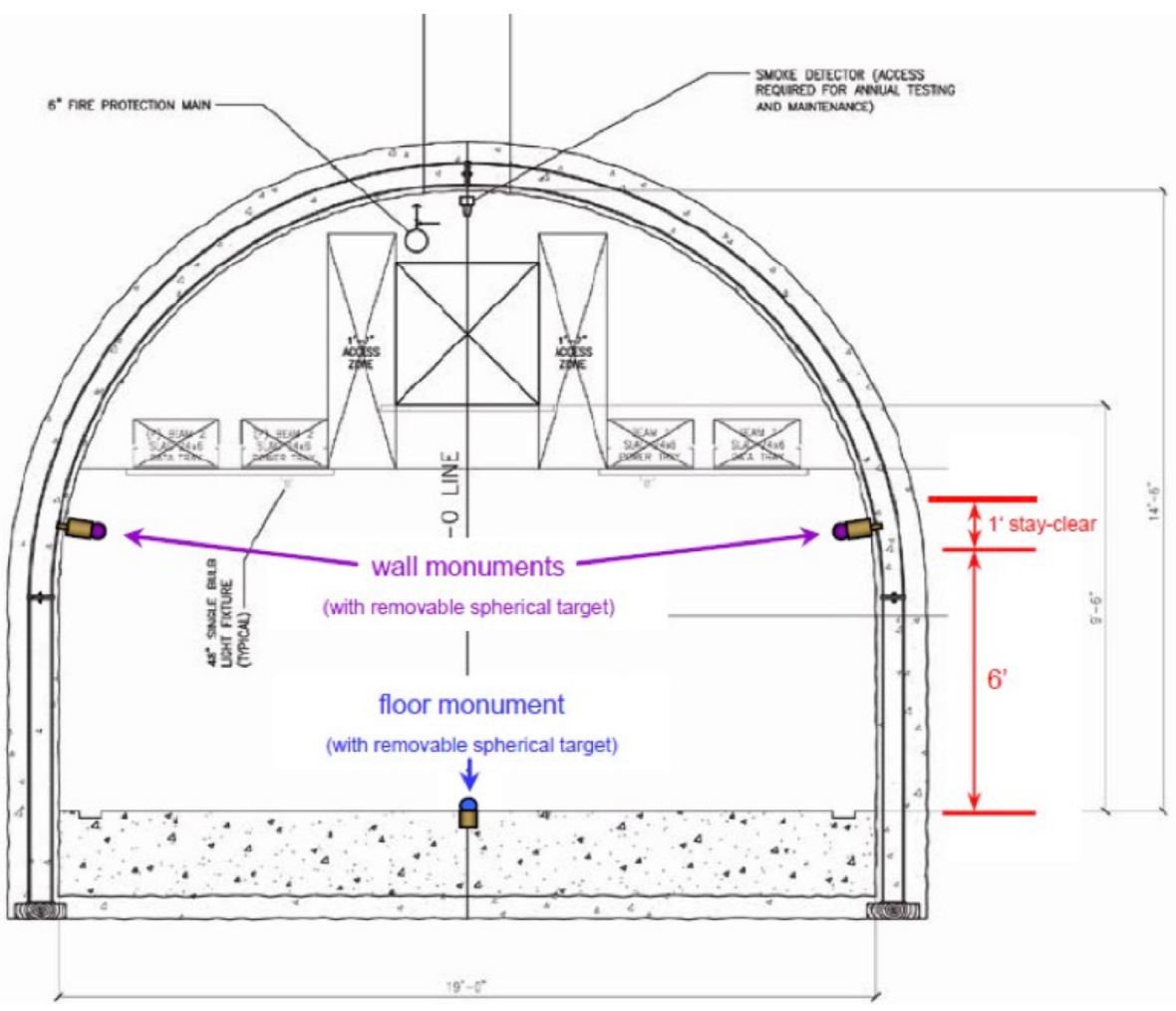

Figure 1.3: Tunnel section with wall and floor monuments forming the tunnel network helenejapon.

The fiducialisation and alignment of the accelerator elements on a common support is the third task. The fiducialisation process relates the internal effective Electro Magnetic (EM) axis of an accelerator component to external marks that are accessible - either visible or touchable - by the surveying instruments. Such targets are then referenced to their nominal coordinates. For example, the ferromagnetic poles from which most of the magnets are made, were often used as reference for external alignment fiducials. The magnetic measurements performed in recent years improved the way to establish fiducials on the magnets. According to the required accuracy, three types of fiducialisation measurements are considered:

- mechanical measurements using a gauge, typically used for warm magnets;

- laser tracker for requirements in the order of $0.1 \mathrm{~mm}$;

- and measurements using a Coordinate Measuring Machine (CMM) for requirements in the order of microns.

As a fourth step, the initial alignment of the girders or other support structures, where the accelerator components are assembled, takes place. The girders are pre-assembled and pre-aligned in a factory before they are transported into the tunnel. This way, the position of the components that are mounted on a common girder can be referenced to the girder coordinate system. 


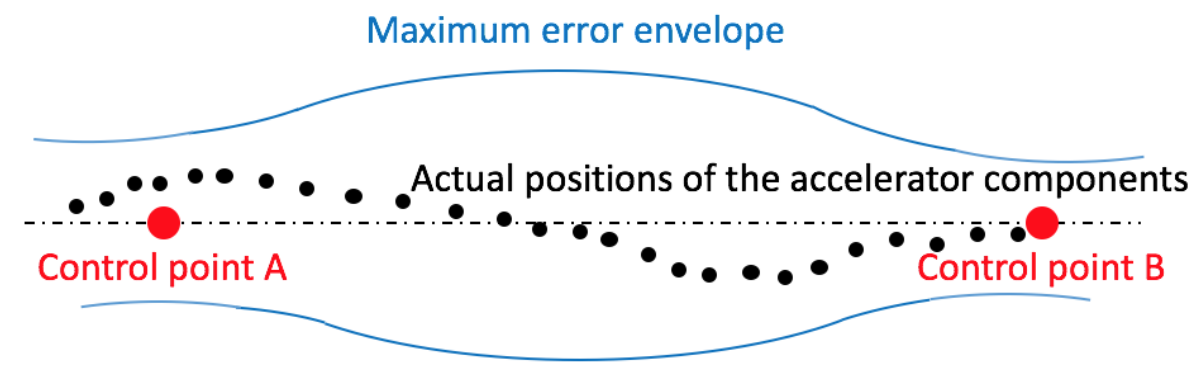

Figure 1.4: Position of the accelerator elements with respect to the theoretical orbit with error envelop.

Fifth, all components are aligned with respect to the underground geodetic network, the so-called absolute positioning step. In this step, the first task lies in the definition of a blue survey line to lay the positions of the anchor bolts in view for the installation of the support systems. A total measurement station, like the Leica TDA500, performs polar measurements with a precision of a tenth of a millimetre with respect to the local transverse points. After the blue line survey, the anchors are set and the pre-aligned girders and components are installed. Moreover, each component is equipped with an adjustment systems to allow a fine tuning of its position. The components are pre-aligned by tapping them into the position exploiting the slack between the anchor bolts and the support structure. The position adjustment values are determined by comparing the actually measured coordinates to the theoretical values. Then, a fine absolute positioning of each component is performed. The components are first set to their ideal elevation with zero pitch and roll using a differential levelling. The horizontal positions are set relative to the tunnel monument system. The components are moved accurately using mechanical adjustment systems. The achievable position accuracy depends on the absolute position accuracy of the reference tunnel network points. For instance, for small scale projects, an absolute alignment of $0.3 \mathrm{~mm}$ is achievable, while for large scale projects, the uncertainty could be in the $\mathrm{cm}$ range. Though, while the absolute position uncertainty might be in the $\mathrm{cm}$ range, the relative position uncertainty over a short distance can still be around 0.1-0.5 mm. After the absolute positioning of girders and components is completed, a quality control survey is performed to verify the results.

The sixth and final step, the smoothing process, involves the relative alignment of adjacent components in order to reduce the local tolerances. Accuracies of around $0.1 \mathrm{~mm}$ are resolved. Taking again the LHC as an example, a relative radial and vertical accuracy of $0.15 \mathrm{~mm}$ over a distance of $150 \mathrm{~m}$ is obtained. This exercise corrects both, the residual errors from the initial positioning of the accelerator components, and from ground motion. In Figure 1.4, we see that the error is at minimum at the control points A and B, and reaches a maximum in the midpoint between two successive control points.

The outlined alignment procedure allows each accelerator component to be located at the foreseen location, which in most cases is chosen such that the beam trajectory passes through the centre of the components. Misalignment of the accelerator elements, e.g. transverse ( $\mathrm{x}, \mathrm{y})$ displacement or pitch, roll, jaw errors causes the beam to travel offcentre, thus receiving guiding or accelerating fields different from those foreseen, which 
creates undesirable effects, like emittance deterioration and luminosity disruption, among other effects [4]. For example, the effect on a misaligned quadrupole translates to a dipolar kick of the beam, and a rotation could cause a coupling between horizontal and vertical beam planes. Also, a beam entering a cavity Beam Position Monitor (BPM) off-centre excites a dipole mode which can be used to determine the beam position. Thus, a misalignment, e.g. xy-offset, of those BPMs lead to wrong beam trajectory determination. A bunched beam traveling off-centre through an AS induces an unwanted EM field -known as wakefield- from the interaction of the beam field with the conductive walls of the AS. As a consequence, the electric and magnetic fields seen by the following charged particles are altered, and may lead to a degradation of the beam quality. Therefore, an excellent alignment of the components meeting the specified tolerances of the accelerator design and during manufacturing is crucial.

\subsection{Alignment in Future Linear Colliders (FLC): ILC and CLIC}

The HEP and the accelerator physics community support the idea that the next future collider to be constructed should be an electron-positron $\left(\mathrm{e}^{-}-\mathrm{e}^{+}\right)$collider in order to explore the laws of nature, focusing on the properties of the Higgs boson. Colliding elementary particles allows the measurement of their internal structure with more precision, as the original states are well defined. In a linear collider, two linear accelerators - or linacs boost the $\mathrm{e}^{-}$and $\mathrm{e}^{+}$beams traveling in opposite directions to their final energy in a single pass, and focus each beam to a head-on collision in a central interaction point. Consequently, an efficient RF system is required to transfer the energy to the beams in a single passage throughout the accelerating chain. The final energy determines the cost and the size of the linac ranging from a few meters to some kilometres.

Two projects for a future electron-positron collider have been proposed, whose feasibility has been studied over the last two decades: the Compact Linear Collider (CLIC) and the International Linear Collider (ILC). The International Committee for Future Accelerators (ICFA) decided in June 2012 that both CLIC and ILC projects should work together solving common technical issues under a strong Linear Collider Collaboration (LCC). The main parameters of ILC and CLIC are summarised in Table 1.1.

\begin{tabular}{|l|c|c|c|c|}
\hline Parameter & ILC & CLIC & CLIC & CLIC \\
\hline Center-of-mass-energy, $E_{C M}[\mathbf{G e V}]$ & 500 & 380 & 1500 & 3000 \\
\hline Luminosity, $L\left[10^{34} \mathrm{~cm}^{-2} s^{-1}\right]$ & 1.8 & 0.9 & 1.4 & 2 \\
\hline Number of bunches per train, $N_{b}$ & 1312 & 352 & 312 & 312 \\
\hline Beam size at IP, $\sigma_{x} / \sigma_{y}[\mathbf{n m}]$ & $474 / 5.9$ & $149 / 2.9$ & $60 / 1.5$ & $40 / 1$ \\
\hline Bunch length, $\sigma_{z}[\mu \mathbf{m}]$ & 300 & 70 & 44 & 44 \\
\hline Acceleration gradient, $G_{a}[\mathbf{M V} / \mathbf{m}]$ & 31.5 & 72 & $72 / 100$ & $72 / 100$ \\
\hline
\end{tabular}

Table 1.1: Main parameters of ILC and CLIC. 
CLIC is a study of a linear collider with a nominal centre-of-mass energy of $3 \mathrm{TeV}$, and a luminosity potential of $2 \times 10^{34} \mathrm{~cm}^{-2} \mathrm{~s}^{-1}$ using $12 \mathrm{GHz}$ normal-conducting AS with an acceleration gradient of $100 \mathrm{MV} / \mathrm{m}$. The Conceptual Design Report (CDR) [4] was published in October 2012 and records the feasibility of a fully optimised $3 \mathrm{TeV}$ electronpositron collider. This project is facing several challenges: high accelerating fields required in the main linac to limit its length and cost, an outstanding beam quality to achieve a high rate of physics events in the detector, and a moderate power consumption of the accelerator complex, thus, a high efficiency to generate RF power to be transferred to the beams. A successful test program has recently been completed at CLIC Test Facility 3 (CTF3) at CERN, see Figure 1.5, to demonstrate the main CLIC concepts, such as operation of high-gradient AS using a drive beam for power generation and experience with advanced beam-dynamics techniques. The current goal is to produce a detailed project development plan of both, the accelerator and detector within the next two years. The baseline AS design will be improved and more statistics on the performance will be obtained. A re-baselining, based on already demonstrated performances of CLIC's novel accelerating technology, was published [5] in August 2016. It proposes a staged construction and operation of the machine, delivering centre-of-mass energies of $380 \mathrm{GeV}$, $1.5 \mathrm{TeV}$ and $3 \mathrm{TeV}$.

The ILC is a project for an electron-positron collider delivering a nominal centre-of-mass energy of $500 \mathrm{GeV}$ and a luminosity of $1.8 \times 10^{34} \mathrm{~cm}^{-2} \mathrm{~s}^{-1}$ using $1.3 \mathrm{GHz}$ superconducting RF technology at $31.5 \mathrm{MV} / \mathrm{m}$. The construction plan and matured technology design of the ILC, was published in the Technical Design Report (TDR) [6] in June 2013. The critical technical demonstrations have been performed in the Beam Test Facilities. For instance, the accelerating gradient demonstration took place at the European XFEL. Precision beam handling and beam dynamics are being studied in the Accelerator Test Facility (ATF) at the High Energy Accelerator Research Organisation (KEK) in Japan. The research held at the Cornell Electron-Positron Storage Ring Test Accelerator (CesrTA), aims to achieve ultra-low emittance beams, the suppression of beam instabilities and the demonstration of the ultra-low vertical emittance operation.

In summary, the common technical challenges facing the ILC and the CLIC projects are: the production of low emittance beams, the preservation of the emittance along the linac, the final focusing of the beams to the nanometer scale, and finally the collision of these extremely small beams with good reproducibility.

\subsubsection{Beam-based alignment techniques in linear colliders}

The quality of the beam in linear colliders may degrade, among others reasons, by: dispersive effects due to misaligned quadrupoles, by $x-y$ coupling created by rotated quadrupoles, and by wakefield generated kicks caused by misaligned accelerating structures. The six-step alignment procedure seen in the first section is not sufficient and active alignment with beam is also required. Table 1.2 shows the alignment tolerances required before beam-based alignment to achieve the anticipated beam quality for the ILC and CLIC, keeping the emittance growth below a tolerable value. These tolerance values apply to all types of components, some are still beyond the state of the art for both colliders. Particular attention needs to be paid to the pre-alignment of the components during 


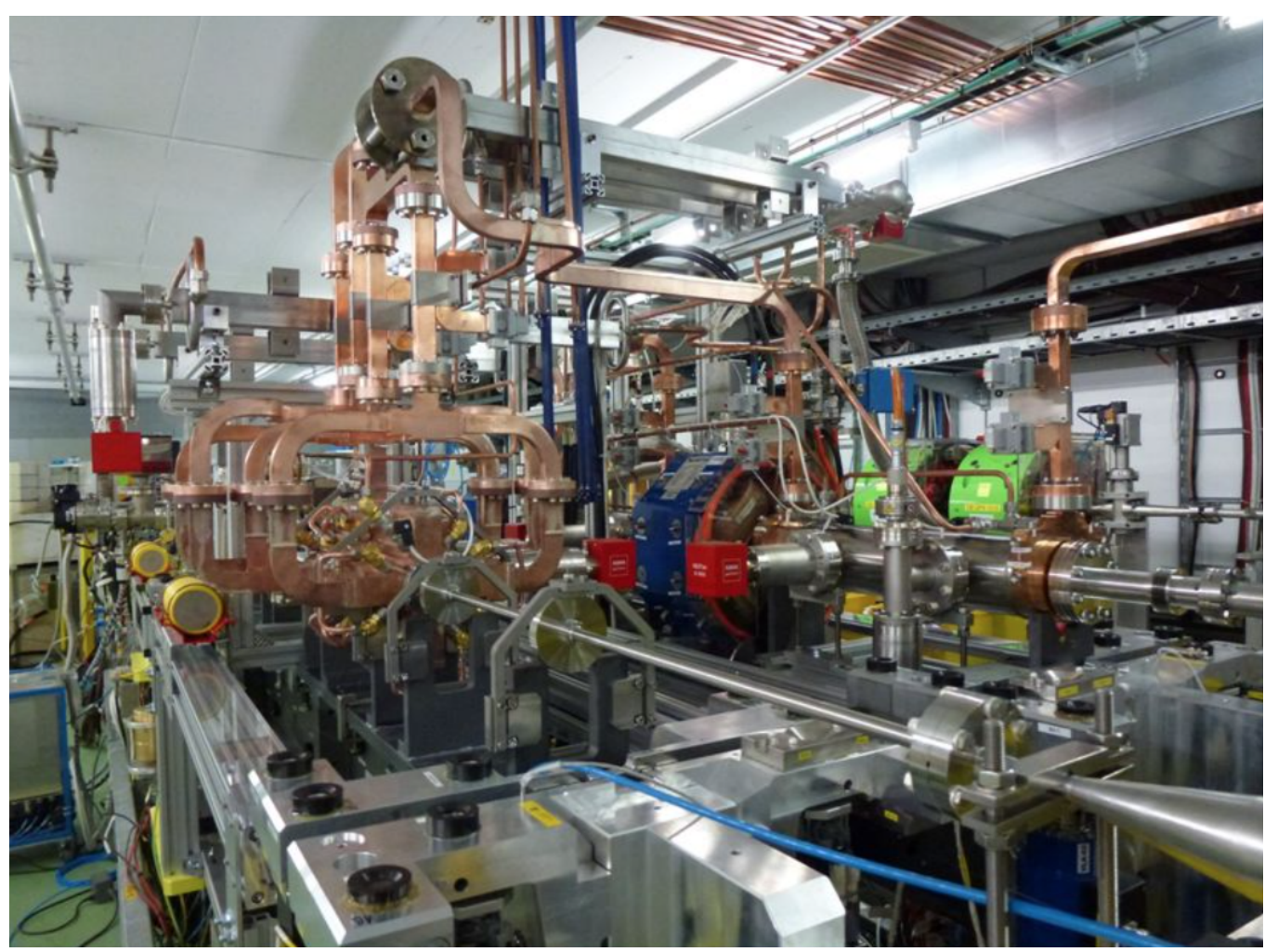

Figure 1.5: The CTF3 facility to verify power production and energy gain while operating at the nominal gradient of around $100 \mathrm{MV} / \mathrm{m}$.

manufacturing and to their alignment during installation.

\begin{tabular}{|l|c|c|}
\hline Parameter & ILC & CLIC \\
\hline Structure offset $[\mu \mathbf{m}]$ & 300 & 10 \\
\hline Structure tilt $[\mu \mathrm{rad}]$ & 300 & 140 \\
\hline BPM offset $[\mu \mathbf{m}]$ & 300 & 14 \\
\hline BPM resolution $[\mu \mathbf{m}]$ & 1 & 0.1 \\
\hline Quadrupole offset $[\mu \mathbf{m}]$ & 300 & 17 \\
\hline Quadrupole roll $[\mu \mathrm{rad}]$ & 300 & 100 \\
\hline Girder offset $[\mu \mathbf{m}]$ & 200 & 9.4 \\
\hline Girder tilt $[\mu \mathrm{rad}]$ & 20 & 9.4 \\
\hline
\end{tabular}

Table 1.2: Alignment tolerances of ILC and CLIC accelerator components and girders.

During the initial installation stage of the beamline elements, the positioning and orientation is performed roughly, accepting rather big alignment errors. The tolerances for cavity offsets and quadrupole rolls can be achieved mechanically but the effects of the quadrupole and BPM offsets need to be reduced.

A BPM for horizontal and vertical beam position monitoring and micron-level singlebunch resolution is located adjacent to each quadrupole magnet. Once a beam passage is settled, a one-to-one steering procedure is applied. It consists on zeroing the reading 
of the BPMs using corrector dipoles upstream. This procedure eliminates the dispersive effects from quadrupole magnet offsets and cavity tilts, assuming the BPMs are perfectly aligned. However, BPM alignment errors are present and, in order to preserve the beam emittance, Beam Based Alignment (BBA) algorithms are required. BBA consists of steering the beam in a dispersion-free path through the centres of the quadrupoles, either by physically moving the magnets or by using beam bumps with corrector dipoles close to the quadrupoles. Two methods are considered whose success lies on the resolution of the BPMs.

The first one is called Dispersion-Free Steering (DFS), here the beam trajectories are measured for different beam energies [7]. The final trajectory minimises the difference and therefore, minimises the dispersion. A variant of DFS has been considered for implementation in the ILC nicknamed Dispersion-Matched Steering (DMS).

The second method is the Kick Minimisation (KM). The BPM offset associated to a quadrupole is measured when varying the quadrupole strength. In the following, the result is applied in a second pass to find a solution for the beam trajectory where the total kick originated from quadrupoles and correctors on the beam is minimised. In other words, the beam trajectory is measured by varying the strength of each quadrupole until the beam passes reasonable close through the centre of all quadrupole magnets, and no orbit change is observed. In the final step a beam-based tuning procedure is applied to minimise the beam emittance with closed-orbit bumps, or by powered tuning magnets that generate specific aberrations, such as dispersion.

The generation of wakefields in warm AS can be minimised afterwards by measuring the beam offset in the structures using novel Wakefield Monitors (WFM) and by readjusting the position of the AS. This process is called RF alignment and is shown in Figure 1.6. Finally, emittance tuning knobs are used to reduce the emittance growth by cancelling the wakefield effects globally by moving AS at various locations. Simulations demonstrate, applying these procedures the emittance growth along the linac will stay within the specifications below $5 \%$.

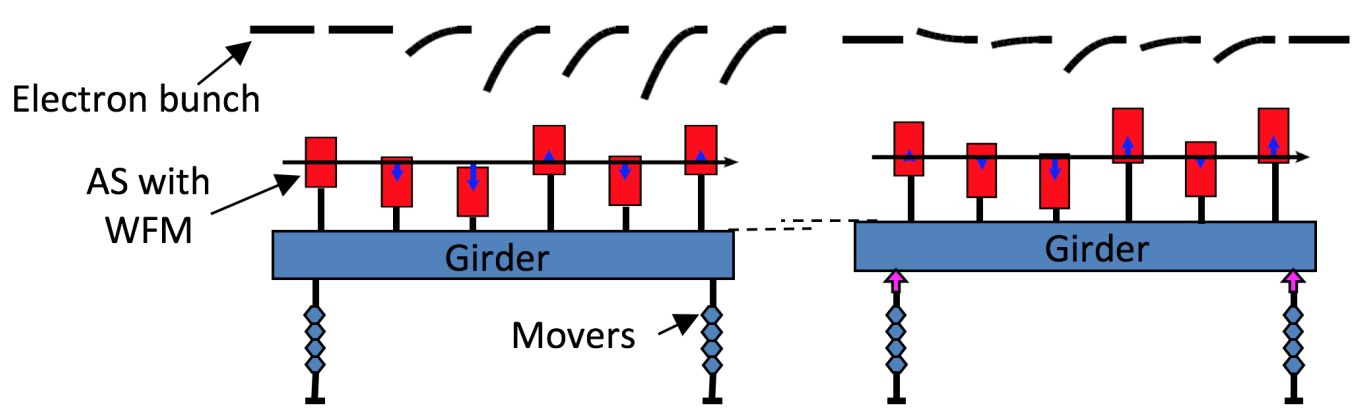

Figure 1.6: RF alignment in CLIC to minimize wakefields in a module with movers and 6 AS. On the left, the offsets AS kick the tail of the bunch. The offset is measured with WFM installed in each AS. On the right, the position of the AS are corrected in order to compensate the kick effects at each AS [8]. 
Keeping the achieved emittance stable in the presence of ground motion is crucial for a proper operation of the machine. Ground motion due to natural and technical sources may cause the beam-guiding quadrupole magnets to move, knocking the beams out of focus and cancelling the benefits of beam based alignment. Active stabilisation of the quadrupoles along the linac in the nanometre range with an active feedback system has already been demonstrated experimentally [9]. Stabilisation of the final focusing doublet in the sub-nanometre regime, to enable beam collisions with high luminosity, is even more challenging in the rather "noisy" environment of the detectors. Applying active alignment, the position of the components is continuously monitored by alignment sensors and is readjusted to the theoretical value by actuators.

\subsubsection{ILC versus CLIC alignment strategy}

As shown in Table 1.2, the alignment tolerances of the ILC are somehow more relaxed compared to those of CLIC, except for the beam delivery system. There are however fundamental differences between them.

Because of the higher power consumption using normal conducting accelerator technology, the beam pulse length of CLIC is short, $156 \mathrm{~ns}$ compared to $1.6 \mathrm{~ms}$ for the ILC. This means, there are fewer number of bunches in each train (see Table 1.1), i.e. lower beam power, but to reach similar luminosity, the beam size at the IP, and therefore the emittance needs to be smaller in both planes $\left(\sigma_{x} / \sigma_{y}=40 / 1 \mathrm{~nm}\right.$ with respect to $\left.474 / 5.9 \mathrm{~nm}\right)$. This puts larger constraints on the alignment for CLIC, compared to the ILC. Moreover, the aperture of the RF cavities of the ILC is substantially larger compared to CLIC, given the different frequency. As a consequence, wakefield effects are more important in the case of CLIC and need to be minimised to a larger extent by aligning the AS more accurately. The largest wakefield amplitude allowed for CLIC has been calculated in such a way that wake affects only the first following bunch, and the wakefield impact from bunch to bunch is controlled.

Another fundamental difference is the fact that in ILC cavities and quadrupole magnets are no longer accessible once installed into the cryomodules. A careful alignment during assembly has to be applied for 850 cryomodules of the main linac, while taking thermal contractions of the support during the cryostat cool down into account. Several stretchedwire studies have proven that the achievements of the alignment tolerances over several thermal cycles is achievable. Stretched-wire sensors monitor the cold-mass displacement and positional reproducibility between cool downs to qualify the alignment.

Even if wakefields are less critical for the ILC, the cavity design includes a Higher-OrderMode (HOM) damping system to suppress any resonate build-up of the wakefields and their effect on the beam. The HOM frequencies are effectively detuned cavity-to-cavity due to geometric differences within the fabrication tolerances. The resulting multi-bunch emittance growth due to cavity misalignment is expected to be below $\mathbf{0 . 5} \mathbf{~ n m}$.

While ILC deals with the alignment of cryostats, the configuration along the CLIC linacs consists of repeated modules and each of both main linacs contains more than 10,000 of them. Five different types of modules are required. All modules contain both, drive 
beam and main beam components, plus a variety of auxiliary systems for vacuum, water, etc. Module type 0 contains only RF structures, and module types 1 to 4 contain beam quadrupole of variable length replacing thus from 2 to $8 \mathrm{AS}$ respectively. The module components are mounted on alignment girders. The length of the module is $2010 \mathrm{~mm}$ and is determined by the length of the AS - $230 \mathrm{~mm}$ - and the fact that each PETS feeds two accelerating structures.

The demanding alignment tolerances of CLIC have lead to the development of an advanced pre-alignment solution to determine the position of the accelerator components that also could be applied to ILC. The pre-alignment technique proposed in the CLIC CDR consists out of three steps [10]. The Metrological Reference Network (MRN) [11] is the primary network, consisting of overlapping stretched wires, each approximately $200 \mathrm{~m}$ long, where the precision alignment is required, and is propagated over the entire $20 \mathrm{~km}$ length of the accelerator. The Support Pre-alignment Network (SPN) is a secondary network framed by the MRN network, that associates sensors to each support to be aligned. The precision provided is a few microns with an accuracy over $10 \mathrm{~m}$. As third step, the Alignment and Fiducialisation of each Component (AFC) on the supports is performed [12]. The final alignment of the components must be perfectly determined with respect to the mechanical interface of the sensors of the SPN network. The solution proposed in the CDR consists of stretched wires with Wire Positioning Sensors (WPS), and is shown in Figure 1.7.

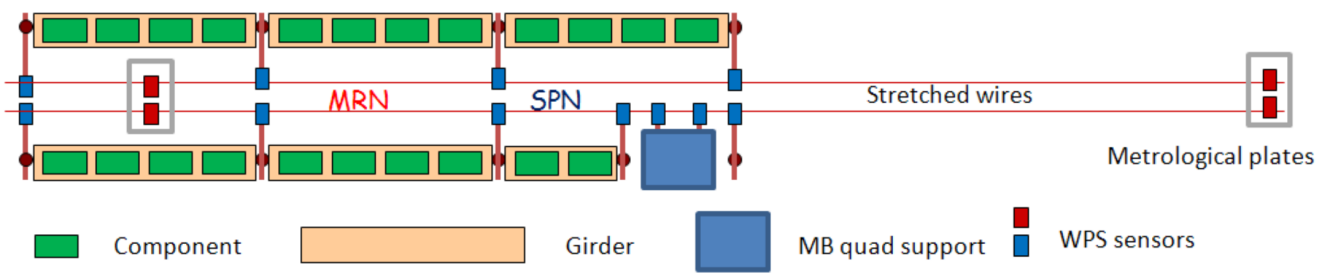

Figure 1.7: Alignment solution for CLIC CDR [10].

Once the position of the components is known, the re-adjustment step can take place. Two types of supports are considered: drive beam and main beam girders. They are interlinked at their extremities by an articulation point, allowing a movement in the transverse girder interlink plane with 3 degrees of freedom, while the longitudinal direction is adjusted with a micro-metric mechanical guiding system. The main beam quadrupole is mounted on an interface plate granting an adjustment along 5 degrees of freedom. An initial mechanical alignment is performed in order to block the longitudinal axis. Cam movers adjust the interface plates and linear actuators adjust the girders.

Several successive mock-ups have demonstrated that sub-micrometric active pre-alignment is possible. Sensors and actuators have been proposed and validated to operate in the micro-meter regime at the CLIC Test Facility 2 (CTF2) at CERN during past years. In the Transfer Tunnel 1 (TT1) facility at CERN, an MRN consisting of overlapping wires and the SPN strategy of a module mock-up have been validated. Alternative optical solutions for the determination of positions are under development. At present, the only solution to validate a stretched wire solution over long range is the cross-check with other stretched 
wire solutions. Another solution under study is the LAMBDA for Laser Alignment Multipoint Based Design Approach. Here, a laser beam under vacuum is used as reference for the alignment. Several sensors are distributed along the beam, coupled to the support to be aligned and determining their offset with respect to the beam. Also, more robust WPS are under development, as well as wires with high rupture force, low linear mass, no creep effect and radiation tolerant. The optimisation of sensors is also under improvement and is based on low cost capacitive sensors in collaboration with the company FOGALE Nanotech [13].

\subsection{The PACMAN project as a novel pre-alignment solution}

The demanding alignment tolerances of the accelerator components for the next generation of linear colliders requires the development of more precise and accurate alignment techniques than currently existing. New techniques should minimise costly and lengthy alignment procedures of independent fiducialisations of single components, such that the accumulation of all systematic and random alignment errors still stay within the projected target.

The alignment requirements provided in the CLIC Conceptual Design Report [4] are based on the mechanical axis of the main accelerator components, namely quadrupole magnets, BPM and AS. The alignment of 3992 quadrupole magnets, 3992 BPM and 142812 AS necessary to build the accelerator, is simplified by grouping them in girders. A total of $8 \mathrm{AS}$ will be mounted and aligned in the same 2-meter-long support, and each quadrupole will be combined with a BPM on another type of support [7]. First tests concerning the current pre-alignment strategy have shown that the accuracy required for future linear colliders like CLIC of $10 \mu \mathrm{m}$, can be reached with this serial process, but its implementation is time consuming, which makes this procedure unpractical to be applied to a large number of units.

In this context, the Particle Accelerator Components Metrology and Alignment to the Nanometre scale (PACMAN) project [14] was funded by the European Commission 7th Framework Programme Marie Curie actions under grant agreement PITN-GA-2013-606839. The technical aim of the project is to improve the alignment precision and accuracy of accelerator components by proposing and developing an alternative solution integrating all the alignment steps and a variety of technologies at the same time and location, for a time efficient alignment procedure with the required precision and accuracy.

CERN hosts the PACMAN Innovative Training Network (ITN) to provide high quality training to ten Early Stage Researchers (ESR) working towards a Ph.D. The human goal of the program is to create a new generation of scientists equipped with a wide-ranging expertise in advanced engineering and instrumentation. It is a multi-disciplinary project to develop new techniques to measure magnetic and microwave fields, optical and noncontact sensors and survey methods as well as high accuracy mechanics, nano-positioning and seismic sensors. The co-operation between the most innovative universities, laboratories and industries of Europe in these fields is required in order to achieve the technical and scientific goals of PACMAN, through secondments, tailored trainings and academic 
supervision. The list of the network partners is the following: CERN, Switzerland; Cranfield University, United Kingdom; Delft University of Technology, Netherlands; ETH Zurich, Switzerland; IFIC, Spain; LAPP, France; University of Sannio, Italy; University of Pisa, Italy; Technical University of Liberec, Czech Republic; SYMME, France; DMP, Spain; ELTOS, Italy; ETALON, Germany; Hexagon Metrology, Germany; METROLAB, Switzerland; National Instruments, Hungary; SIGMAPHI, France; and TNO, Netherlands. Their distribution around Europe is shown in Figure 1.8.

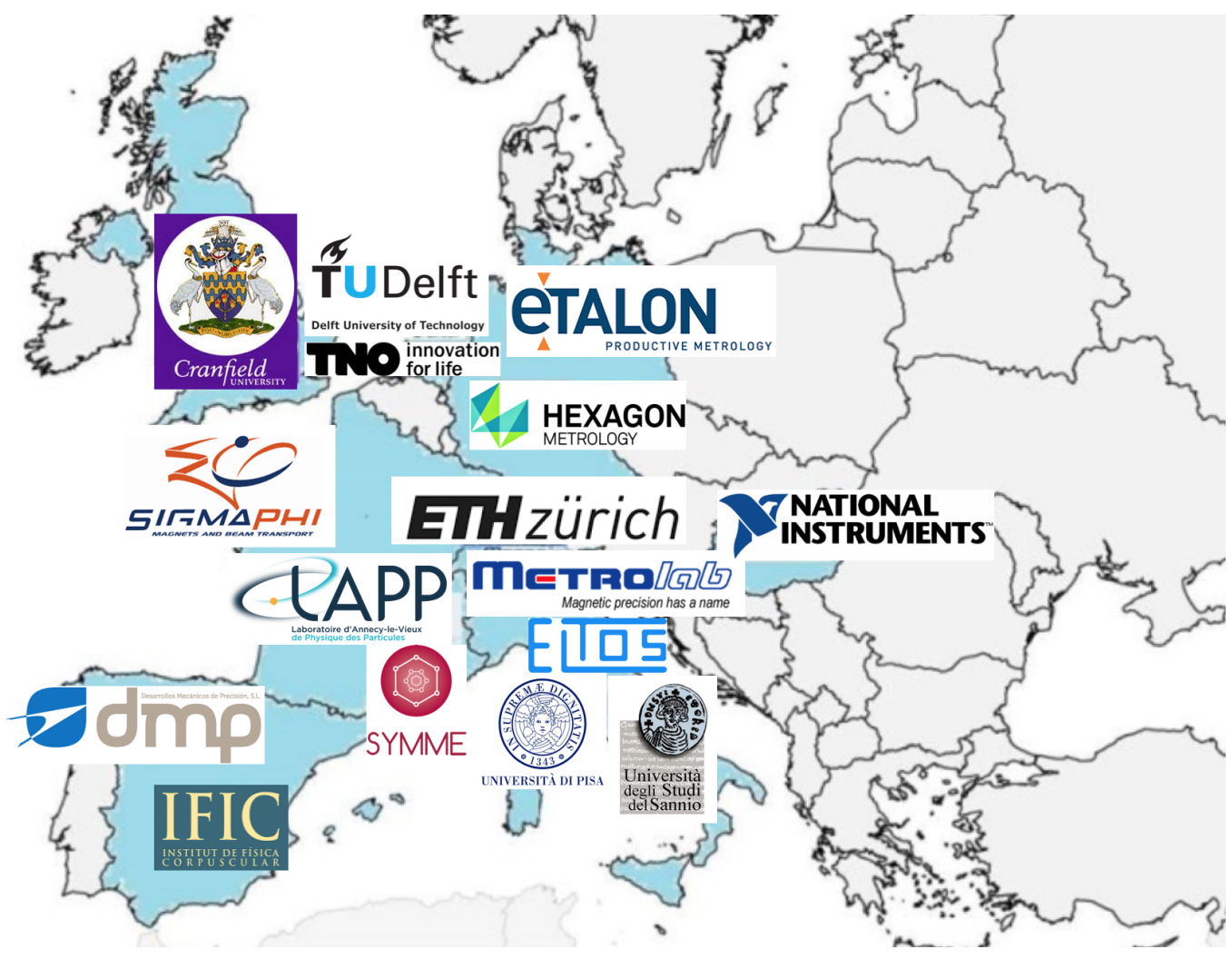

Figure 1.8: PACMAN network composed by the list of associated partners.

\subsubsection{The pre-alignment strategy of PACMAN}

The strategy of PACMAN is to regroup the pre-alignment steps at the same time and location [15]. The first step is the fiducialisation of the components and their supports. The fiducialisation process addresses the position of the reference axis of the components with respect to external alignment targets, named fiducials. The second step is the pre-alignment, or initial alignment, of components on a common support assembly. In particular, the pre-alignment of sensors interfaces which will be used later to align the components at their theoretical position in the tunnel general coordinate system when their reference axes are not accessible anymore. PACMAN will allow the fiducialisation of several components of different types simultaneously, in the environment of a threedimension CMM [16] in order to gain time and accuracy in view of the large number of components needed for the accelerator. To ease the process, the cavity BPM and the quadrupole are assembled on the same support, and up to four AS are placed on another common support with respect to their EM centre. At this point, the scope of PACMAN 
is reached and finally, as third step, both supports are transported to the tunnel for their alignment reaching an accuracy of $10 \mu \mathrm{m}$ with respect to a straight line over 200-m-long segments, along the $20 \mathrm{~km}$ of linear accelerators, using stretched-wire techniques.

The PACMAN project started in September 2013 and the new methods developed uses a stretched wire to materialise the reference axis of the components with micrometric accuracy, serving as a reference for the fiducialisation. Notably, the reference axis refers to the magnetic axis of magnets with $5 \mathrm{~mm}$ aperture, the electrical centre of a $15 \mathrm{GHz}$ BPM [18] and the EM axis of the TD24 AS [19] with $5.5 \mathrm{~mm}$ mean aperture. The results obtained concerning the magnetic axis of the quadrupole magnet using a metallic stretched wire are cross-checked by an alternative method based on printed circuit board rotating coils. PACMAN integrates also solutions to characterise and measure the position of the reference wire in the coordinate frame of the common support assembly with respect to the external alignment targets. Accordingly, a three-dimension CMM and portable alternatives based on Frequency Scanning Interferometry (FSI) and micro-triangulation are undertaken. Other systems developed in the frame of the project are also taken into account: a nano-positioning system to validate the nanometric resolution of the BPM and a dedicated seismic sensor to characterise the environment during the measurements. The solution developed within the PACMAN network, or Final PACMAN Alignment Bench (FPAB), should look as sketched in Figure 1.9, needs to be robust and needs to work reliably in an industrial environment.

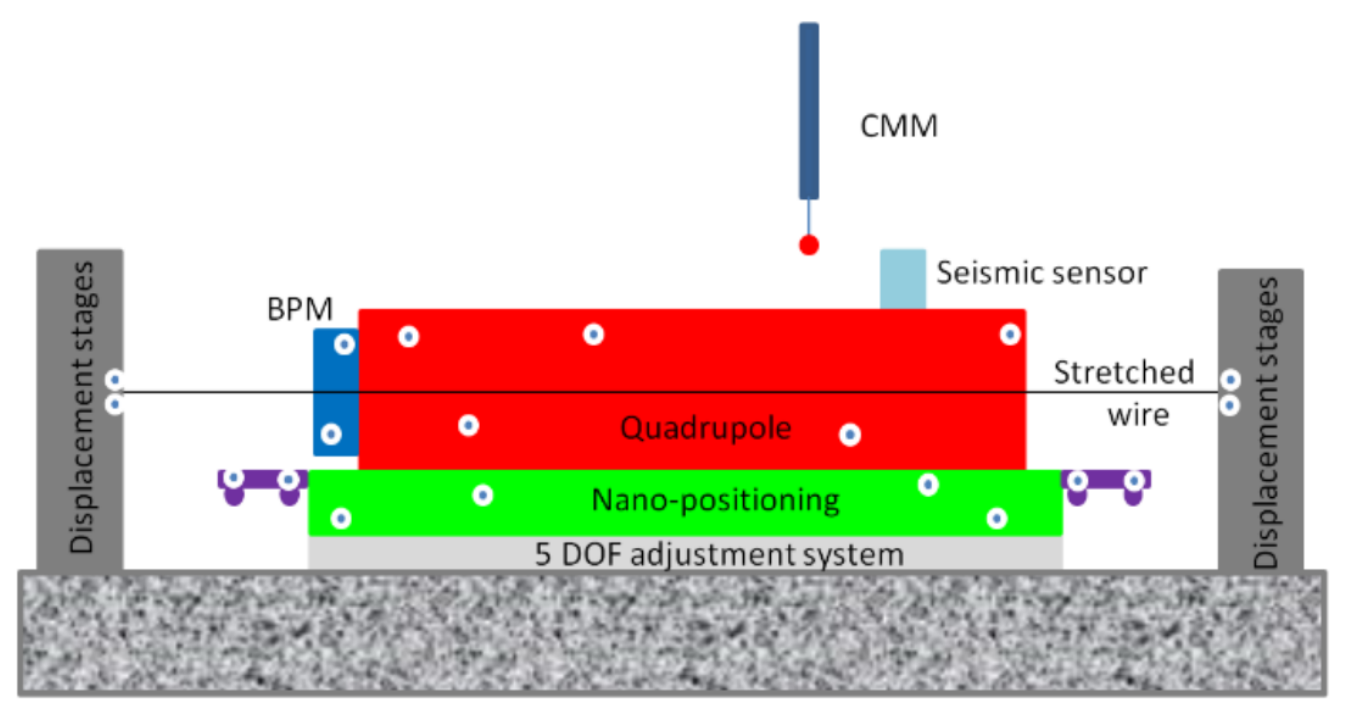

Figure 1.9: Schematic view of the final PACMAN pre-alignment bench.

\subsubsection{Scientific Program of PACMAN}

PACMAN is organized in four Workpackages (WP), each working on different aspects of the alignment [20]: WP1, Metrology and Alignment; WP2: Magnetic Measurements; WP3: Precision Mechanics and Nano-Positioning; and WP4: Microwave Technology. Two or three ESR integrating a WP work together to upgrade or develop a new existing measuring technique to their ultimate precision and resolution, and their integration into 
the prototype alignment bench. The individual subjects of the ESR and the results obtained are summarized below [17].

ESR1.1 sees the development of an optical sensor to be plugged in the Leitz CMM available in the metrology laboratory at CERN. The aim is to provide absolute measurements of the wire in the local coordinate system of the CMM. Repeatability and accuracy on the measurement is required in an environment with magnetic fields. The wire used for metrological, magnetic, electric and electromagnetic measurements is a $\mathbf{1 0 0} \mu \mathbf{m}$ diameter wire made of $98 \%$ copper and $2 \%$ beryllium [21]. Its magnetic and dimensional properties were characterised by ESR1.1. The form error of the wire is a source of uncertainty in the positioning of the wire axis, and it can only be done by measuring a circle on the wire surface. A Shape Evaluating Sensor: High Accuracy and Touchless (SESHAT) was developed to tackle this issue and achieve a measurement uncertainty on the wire shape of $\mathbf{1 0 0} \mathbf{~ n m ~ [ 2 2 ] . ~ T h i s ~ s e n s o r ~ w o u l d ~ d e t e r m i n e ~ t h e ~ p o s i t i o n ~ o f ~ t h e ~ s t r e t c h e d ~ w i r e ~ w i t h ~}$ respect to fiducials by plugging its stator on the head of the CMM. The rotor is moved on high-precision air bearings turning around the wire allowing the form measurement. The components are currently under qualification and assembly. ESR1.1 also performed studies in the Leitz Infinity CMM at CERN in order to probe that the measuring machine was not affected by magnetic fields when a non-contact probe is used [23].

ESR1.2 uses FSI techniques to perform multilateration measurements for the development of a portable metrology method. A network should be built around the FPAB to determine the distance between the wire and the fiducials. The fiducials also need to be developed to be plugged to FSI fibre end to perform absolute distance measurements, being the centre of the fiducial the zero of the fiber. The solution carried out uses $\mathbf{3 8 . 1} \mathbf{~ m m}$ diameter ceramic reference spheres and customized supports. Distance measurements can be performed in different directions from the same point with micrometric repeatability [24] by turning the fiber or the ceramic sphere in which the fiber has been inserted. The optimised multilateration network produced is formed by 8 measurement stations or pilars located on each side of the measurement volume [25]. They make observations to the targets glued on the quadrupole magnet and to the wire support. A high-index glass sphere with a sphericity of $\mathbf{6 3} \mathbf{~ n m}$ has also been developed in order to be placed at positions of open viewing angles [26]. Initial results based on 8 stations and 17 targets in the environment of the CMM presented a measurement uncertainty of $\mathbf{0 . 5} \mu \mathbf{m}$ per metre over a period of $16 \mathrm{~h}$ without including calibrations [27]. Distance measurements from outside the CMM towards targets mounted on the CMM varied below $\mathbf{4} \mu \mathbf{m}$ over a period of $2 \mathrm{~h} \mathrm{[28].}$

ESR1.3 adapts micro-triangulation from the QDaedalus measuring system to determine the position of the wire with respect to fiducials. Horizontal and vertical angle measurements are performed from 4 Leica TDA 5005 theodolites -equipped with QDaedalus systems- to targets accurately, automatic and contactless [29] [30]. ESR1.3 developed two computer vision algorithm with an innovative mathematical triangulation model to facilitate the contactless measurement of the wire -detection [31] and reconstruction [32]and the fiducials. The software created also allows the simulation of various configuration networks before installation [33]. Validation tests on the fiducials and on the position and orientation of the wire in space have been carried out and cross-checked with CMM measurements. An accuracy better than $\mathbf{2 0} \mu \mathrm{m}$ on the wire positioning was found and it 
was around $\mathbf{4 0} \mu \mathbf{m} / \mathbf{m}$ on the wire orientation [34].

ESR2.1 needs to create a magnetic measurement system based on the oscillating wire field-measuring technique for small aperture magnets for alignment purposes. The wire, stretched through the bore of the magnet, oscillates in the magnetic field when it is excited by an alternating current [35]. The magnetic axes is located when the amplitude of vibrations is minimum. The wire is hold by two precision alignment stages mounted on granite supports and it is tensioned by a stepper motor. The accuracy of the wire positioning system has been measured and the repeatability found on the wire repositioning was $\pm 1.5 \mu \mathrm{m}$ [36]. Fiducials were also implemented in order to reconstruct the position of the wire. Two orthogonal stages allow the positioning of the wire with a repeatability below $0.1 \mu \mathrm{m}$ and an absolute accuracy below $1 \mu \mathrm{m}$. The amplitude of the oscillation was measured using optical micrometres orthogonally mounted on one of the wire stages with a repeatability of $\pm 0.3 \mu \mathrm{m}$ and an accuracy of $\pm 0.5 \mu \mathrm{m}$. Finally, an acquisition system was developed in order to control the generated voltage by the optical micrometres that is proportional to the wire displacement. The compatibility of the stretched-wire system with the CMM was also validated [37]. The test was done with the wire static and vibrating under different conditions and no deviation from the normal operation of the system was detected. A second test was done in the CMM with the magnet powered below its nominal working conditions [38]. The magnetic axes found in both conditions had a systematic difference of $\mathbf{3} \mu \mathbf{m}$, so a correction technique was developed [39]. Techniques to correct the systematic error coming from wire saggita were also performed resulting on a correction amount of below $\mathbf{1 0} \mu \mathbf{m}$ on the wire vertical position. ESR2.1 performed an extensive investigation on the system parameters and their influence in the measurement, allowing the localisation of the magnetic axes with submicron repeatability under the same measurement conditions [40]. The repeatability of the measurement of an oscillating wire for a fixed current at the FPAB was $\pm \mathbf{0 . 1} \mu \mathbf{m}$ for the horizontal and vertical coordinates, and it was $\pm \mathbf{1} \mu \mathbf{r a d}$ for the pith and yaw angles.

ESR2.2 works on the development of a magnetic measurement system based on rotating coils using Printed Circuit Board (PCB) technology, for direct comparison with the oscillating wire technique. The customised production and assembly of the PCB was done at CERN ensuring the high precision of the required tasks: the layer alignment, reduced thickness and rigid shaft. Validation tests were successfully undertaken at Fermilab [41], and the calibration on the sensor position [42] resulted on a shift with respect to the design position of $22 \mu \mathrm{m}$ in radial and $\mathbf{6 2} \mu \mathrm{m}$ in the vertical direction.

ESR3.1 is required to devise a new procedure for the assembly of magnets and the positioning of BPMs on their support within the micron level. The full mechanical integration of the prototype alignment bench within the total error budget was also an assigned task for this ESR. Two gaps of knowledge were identified within the studies carried out by ESR3.1 [43]. Several tests were done with the standard ISO 10360 to calibrate the stochastic model of the CMM [44]. The uncertainty on the location of a fiducial in a dimensional reference frame of 3 spheres was from 4 to $8 \mu \mathrm{m}(\mathbf{1} \sigma)$. Thermal error studies have been done using the quadrupole magnet. The variation on the magnetic axis drifted with more than $2 \mu \mathbf{m} /{ }^{\circ} \mathbf{C}$. Empirical and numerical models were developed to predict and compensate the drift with an uncertainty of around 4 to $7 \mu \mathrm{m}(\mathbf{1} \sigma)$. 
ESR3.2 is required to develop a seismic sensor with a bandwidth from 0.1 to $200 \mathrm{~Hz}$, a resolution of $0.1 \mathrm{~nm} \mathbf{~ r m s}$ at $1 \mathrm{~Hz}$, with no influence by external magnetic fields and low noise to measure quiet ground motion. The solution needs to be compact to fit in the FPAB and light-weight to avoid disrupting the measured structures. The solution developed integrates 3 types of sub-nanometre displacement transducers using the same data acquisition and processing: a Fabry-Perot interferometer, an optical encoder and a capacitive transducer. Different resolutions were obtained from all of them when operating under the same conditions, but none of them could achieve the required resolution. The most convenient solution found to be used as a seismic sensor for the requirements of PACMAN was the integration of a multi-pass Michelson interferometer in a configuration with 8 reflections [45].

The task of ESR3.3 is to build the first prototype of a $\mathbf{4}$ degrees of freedom nanopositioning system for alignment and stabilisation in order to be used in the FPAB. The manufactured solution consisted on an optimisation of the CLIC stabilisation system [46], with a reinforced base plate combined with the bolting and gluing of the components in the base region. The side plates were lowered improving the line of sights to the fiducials, and new supports for optical encoders were installed in order to control the lateral motion of the magnet with steps of $\mathbf{5 0} \mathbf{~ n m ~ [ 4 7 ] ~ [ 4 8 ] . ~ T h e ~ s t a b i l i t y ~ r e q u i r e m e n t ~ o f ~} \mathbf{1 . 5} \mathbf{~ n m ~ r m s ~ a t ~}$ $1 \mathbf{H z}$ was achieved with the implementation of an active vibration isolation [49].

The demonstration of the nanometer resolution of the $15 \mathrm{GHz}$ cavity BPM by using an RF excitation on the wire, was assigned to ESR4.1. The absolute alignment of the BPM, a long-term stability of a few micrometer and high temporal resolution were also required. The RF signal excitation method and the wire perturbation method were studied to locate the electrical zero of the cavity. The second method was chosen for integration purposes, and a dedicated test bench was developed in collaboration with ESR4.2 [50] [51] to characterise the cavity BPM. An hexapod allowed the BPM displacement in 6 degrees of freedom with respect to a stretched wire installed inside the BPM. The minimum perturbation of the EM fields created by the wire was related to the centre of a high order dipole mode [52]. The repeatability of the measurements showed to be in the sub-micron range [53]. A piezo actuator developed within the PACMAN network was installed between the BPM and the hexapod, providing a controlled vertical displacement with nanometric accuracy. The resolution found on the BPM was 12 nm [54].

And finally, this Ph.D. thesis has been completed by ESR4.2. The objective was the investigation of a non-destructive technique to measure the axes of AS and an experimental validation on the TD24 AS CLIC prototype CLIC. The details on the solution proposed and the results are presented in the next chapters.

The concepts and tools developed by the PACMAN team to determine the magnetic axes of a quadrupole magnet and the electrical axes of an RF cavity BPM were firstly done on dedicated test benches and then on the FPAB developed within this project [55]. The FPAB consists on a nano-positioning system hosting the assembly of a CLIC Main Beam quadrupole, a CLIC RF-BPM and a seismic sensor. The technical systems of the FPAB are shown in Figure 1.10. Once a stretched wire has been used to localise the reference axis of the involved accelerator components, the three methods developed in PACMAN are used to determine the position of the reference axis with respect to the fiducials [56]. 


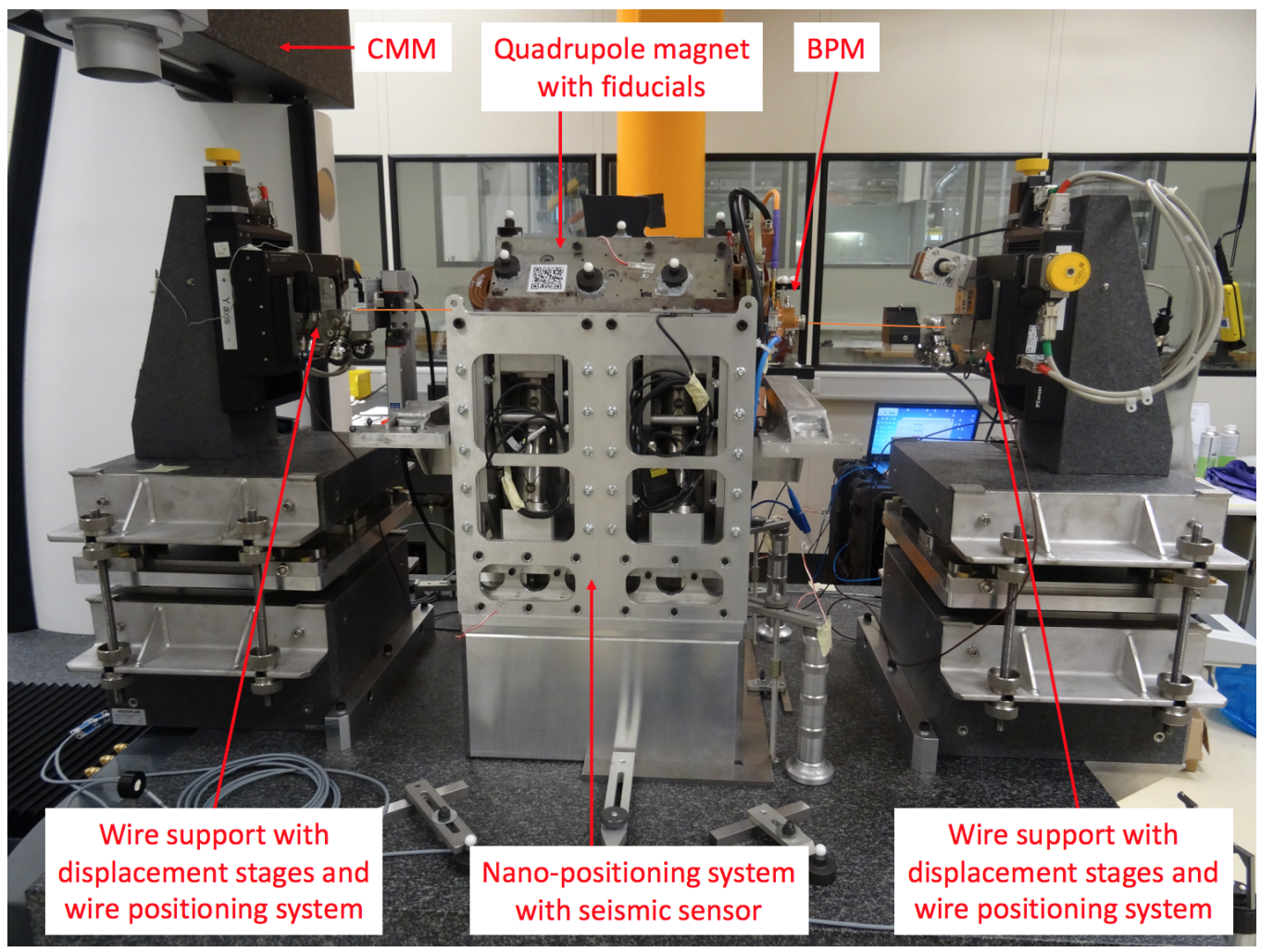

Figure 1.10: Prototype bench developed within the PACMAN project.

The test performed confirm a repeatable fiducialisation of $\mathbf{1} \mu \mathbf{m}$ between the quadrupole and the BPM located on a common assembly using the same wire.

\subsubsection{The innovative aspects coming along the success of the program}

The project is meant to finish by end of 2017 with a first prototype bench, see Figure 1.10, and a full understanding on the limitations of alignment for accelerator components. A lot of the scientific innovations have been accomplished along the three years of the European programme. The tools and methods developed have been successfully validated on dedicated test benches using the CLIC accelerator components.

The work held by the 10 ESRs of the PACMAN project have produced a lot of premieres, and the success of the new solutions proposed may infer in many different cutting-edge forms listed below. The most accurate absolute fiducialisation process of AS, quadrupole magnet and BPM was accomplished; it is the first attempt to transverse alignment on a micrometer scale for objects in the metre range; concerning metrology, for such a volume of measurements, no methods were available for portable absolute measurements in the micrometre range, and the methods developed at PACMAN exploited this aspect in order to be used not only in particle accelerators, but also in other domains such as aerospace and automotive industry; PACMAN is the first network created to combine simultaneously measurement and active alignment of mechanical and EM structures; a BPM resolution below $12 \mathrm{~nm}$ using a stretched-wire was achieved; for the first time, the combination of 
high loads, magnetic field and vibration stabilisation, reaching the level of accuracy required is done using commercially available nano-positioning systems; the manufacturing of the smallest coil in the world; and the production of the first seismic sensor integrating several transducers; the solution is compatible with an EM environment.

The methods and tools developed in PACMAN improve the accuracy coming from the standard alignment strategy of CLIC and can be extrapolated to other accelerator projects. They offer the possibility to carry out fiducialisation and alignment of different types of accelerator components, simultaneously on a common support with micrometric accuracy. In addition, the development of sensors adapted to the severe accelerator environment may suppose a progress on the technology, that are required to deal with magnetic fields, ionising radiation and high electrical and mechanical noise. 


\section{Chapter 2}

\section{New methodology and EM field simulations to measure the EM axis of Accelerating Structures: the case of the CLIC TD24}

In this Chapter, we propose a new non-destructive technique to measure the EM axis of the CLIC TD24 AS by means of a stretched wire in a laboratory environment. First, the features of the TD24 test AS developed for CLIC are described. Second, the different existing methodologies to measure the EM axis of AS and a new technique are explained in detail. Then, an implementation plan is proposed and an extensive EM field simulation study of the structure is performed with the help of an EM field solver. Finally, we exploit the simulation results to develop the conceptual design measurement algorithm within the target accuracy.

\subsection{The TD24 AS developed for CLIC}

The TD24 AS we will use to measure its EM centre is a normal-conducting travelingwave test structure developed at CERN. The T24 AS family is the original candidate conceived in the CLIC project to provide efficiently the constant acceleration gradient of $100 \mathrm{MV} / \mathrm{m}$, working at $12 \mathrm{GHz}$ in the $2 \pi / 3$ mode [57] [58] [59]. The name T24 refers to the number of cells forming the AS. The 3D design of the structure is shown in Figure 2.1, and consists of two coupling cells and twenty-four regular cells whose iris dimensions decrease gradually. This process is known as tapering [60] and is introduced to give the required constant acceleration gradient. The linear tapering of the iris radius and thicknesses provides an optimum distribution of high-power parameters avoiding hot spots, and also implements the detuning of the HOM. The dimensions of the pure copper structure and the iris apertures on the first and last cell can be seen in Figure 2.2. The $12 \mathrm{GHz}$ RF input signal is coupled from cell to cell through the irises with a mean aperture of around $5.5 \mathrm{~mm}$. The D letter of the TD24 name indicates damping of HOM. Four radial waveguides with a cut-off frequency of $15 \mathrm{GHz}$ connect with every cell, see 
Figure 2.3 (a), for HOM damping extracting the power of transverse modes from the structure without distorting the accelerating mode [61]. The basic cell geometry is shown in Figure 2.3 (a) and is adapted to the manufacturing process, being flat in the back side and carrying all the cell features in the front side. The section of the iris is elliptical, with semi-major axes ranging from $0.8690 \mathrm{~mm}$ in the first cell to $0.5103 \mathrm{~mm}$ in cell 24 , and semi-minor axes from $0.7449 \mathrm{~mm}$ to $0.45 \mathrm{~mm}$, respectively. The cell length for optimum cost and RF performance is around $8.3 \mathrm{~mm}$, as shown in Figure 2.2. Four bent waveguides are coupled as an extension of the damping waveguides of the middle cell. They are terminated in an RF load and are equipped with two RF pick-ups just before the load, forming the WFM shown in Figure 2.1 [62].

In CLIC, precisions below the micron are achieved in the fabrication of the individual cells, concerning profile surface, surface roughness $R a$ and flatness by ultra precision machining. The cell tolerances established for each cell during the RF design process are shown in Figure 2.4 and are expressed in mm except for surface roughness, which is typically defined in $\mu \mathrm{m}$. In the figure, the front side is indicated as label A and the back side is indicated as label B. Semi circles indicate profile surface and diamonds indicate flatness. The actual geometry of the cell is verified by measuring the shape of the single cells using topographic contact-scanners, like CMMs, with sub-micron accuracy. The stack of cells is assembled by copper diffusion bonding [63] at a temperature of around 1000 degrees and inside a hydrogen protective atmosphere. Cell stacking is done manually using special tools developed for this purpose [64], and this will determine the internal geometry and eventual misalignment of the final structure. The accepted tolerances for the minimisation of transverse wakefields in the AS after assembly are displayed in Figure 2.5. Iris deformation is very unlikely given the good machining of the independent cells, so no tolerance has been defined. The end tolerance results as a sum of: cavity shape, transversal offset and tilt. This tolerance needs to lie within a few to $10 \mu \mathrm{m}$. Once bonding has taken place, the CMM is no longer suitable for the evaluation of the internal shape of cells stack given the complicated accessibility to the AS through its small irises. Therefore, the internal assembly tolerances can not be validated. The current technique to determine the geometric centre in each cell after bonding relies on the measurement of their outer cylindric surface. If we assume that the geometry of cells is not deformed after bonding, the knowledge of the positioning of the internal geometric centre in each cell resolves the knowledge of the positioning of the EM centre, for instance, by using EM field solvers. If assembly deforms the cell geometry, this calculation does not necessarily coincide with the geometric centre and an intra-cavity technique needs to be developed to verify this assumption. In ideal conditions, they should coincide. In view of this, the main objective of this Ph.D. thesis is to develop a technique to measure the position of the EM centre of the TD24 with a target accuracy below $10 \mu \mathrm{m}$.

In parallel to this research, the Helsinki Institute of Physics is currently developing an alternative to measure the internal geometry of cells using a non-destructive optical device based on interferometer with sub-micron accuracy. The development of a scanning system is also on going to measure simultaneously the internal and external topography of the AS by means of two optical fibers. Satisfactory results are being obtained [65] [66]. 


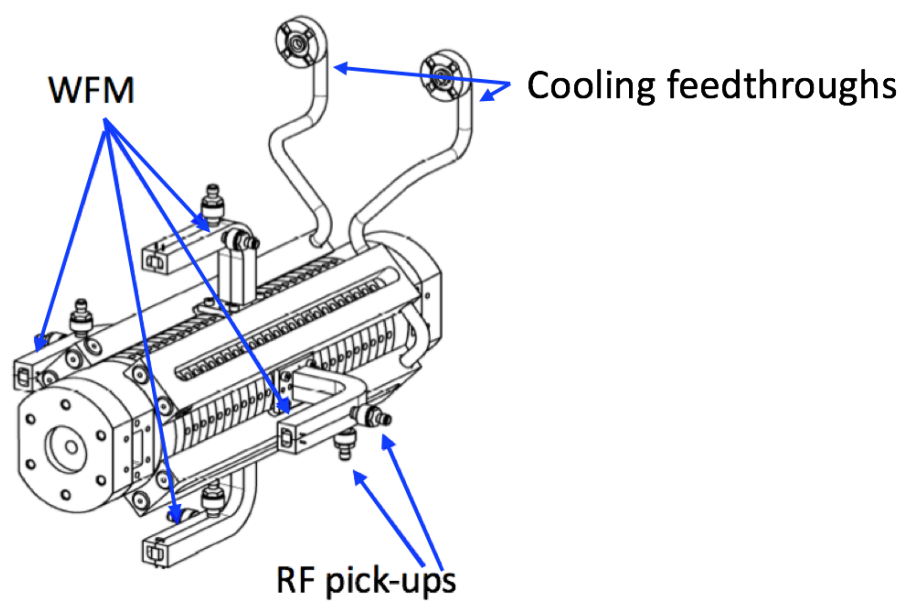

Figure 2.1: 3D design of the CLIC TD24 AS developed for CLIC equipped with four WFM, two RF pick-ups in each WFM and two cooling feedthroughs.

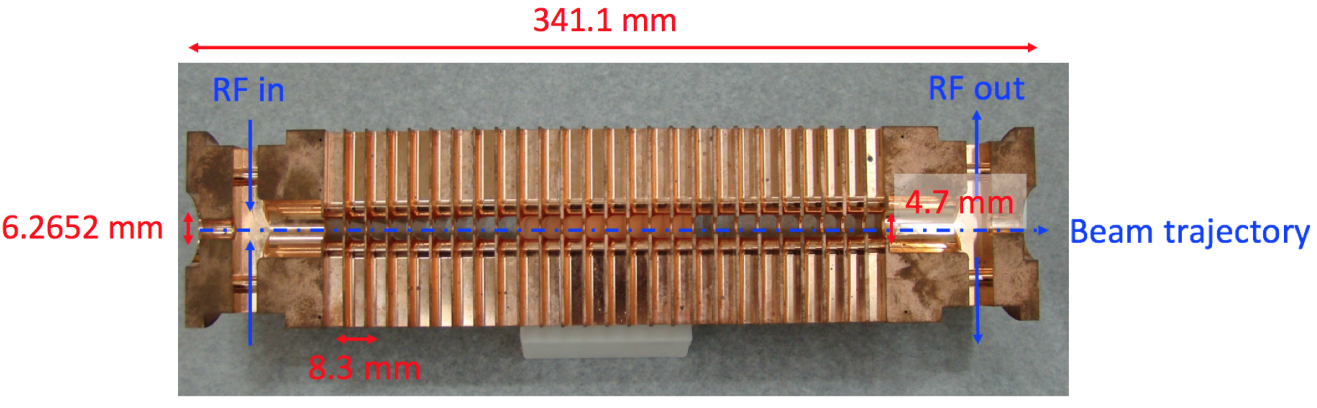

Figure 2.2: Longitudinal cut and dimensions of the TD24.

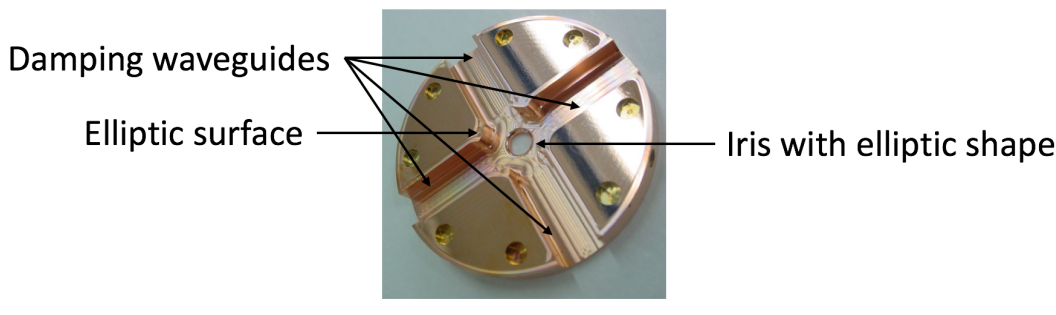

(a)

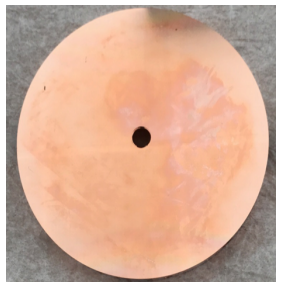

(b)

Figure 2.3: (a) Front side of the middle cell of the CLIC TD24 AS carrying all the cell features and (b) back side. 


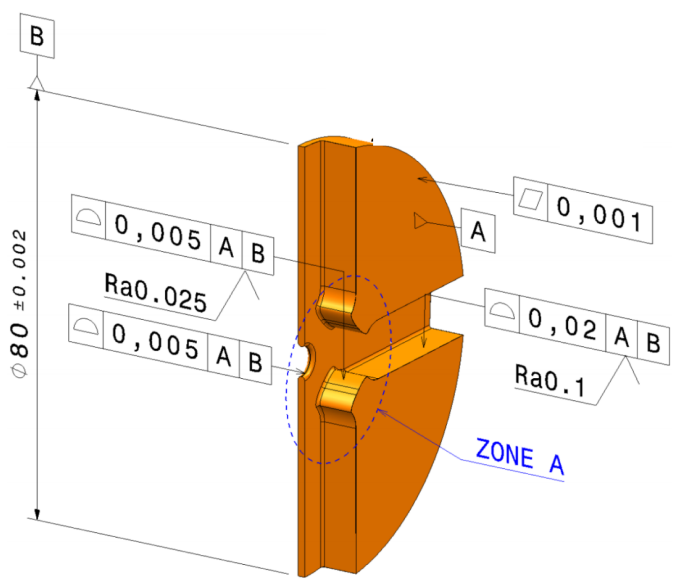

Figure 2.4: Fabrication tolerances of the individual cells expressed in $\mathrm{mm}$.

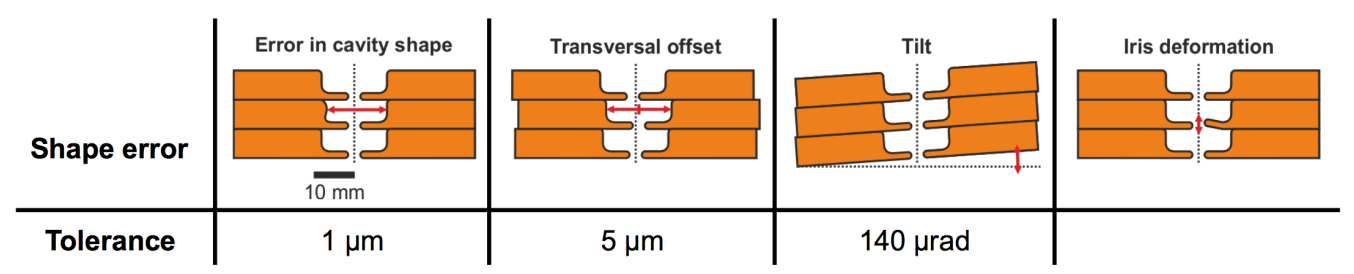

Figure 2.5: Maximum alignment tolerances accepted in the assembly of the CLIC AS.

\subsection{Current technique to estimate the geometric centre of AS}

In this section, we describe the current technique used to estimate the geometric centre of ASs. This technique uses topographic measurements of their outer geometry in order to infer the geometric center, typically using a CMM. To explain the method, we use the metrology measurements of the TD24 performed within this thesis to evaluate the geometric fabrication imperfections of the structure that we will use to measure its EM center.

The geometric center of the TD24 was estimated using the Leitz Infinity CMM [16] in the metrology laboratory at CERN. The uncertainty of this measuring instrument, $E_{0}$, is proportional to the length $L$ of the device under test following:

$$
E_{0}[\mu \mathrm{m}]= \pm 0.3[\mu \mathrm{m}]+L[\mathrm{~mm}] / 1000
$$

The AS was placed in a support in the marble of the CMM and was fixed on top of it with an aluminium tool, as shown in Figure 2.6. The geometric centre of the first matching cell was defined as the origin of the coordinate system, whose axes were defined as shown in the picture. The two alignment blocks at each side of the reference cell were used as alignment references for the coordinate system. Once the reference system was fully settled, topographic measurements were performed all along the surface of the TD24. In particular, the position of the four points indicated in the picture as P1, P2, P3 and P4 around the cell, were measured by the CMM. The position of the geometric centre of the first matching cell was calculated from these four position measurements by the software 
of the CMM. This process was repeated all along the TD24, $L$, and a total of 52 geometric centres were obtained. So, a total of two centres were measured for each cell to allow the observation of a possible book-shelving between the cells. The metrology results are shown in Figure 2.7, where the position of the calculated EM center in $X$ (green dots) and $Y$ (blue dots) are represented. The purple and pink solid lines represent the linear fit of the geometric centre in each axis $X$ and $Y$, respectively, used to position the AS in its girder. The error bars given by the uncertainty of the CMM in Eq (2.1 in Chapter 2$)$ are not included in the plot due to their small value with respect to the range of length that we use in the plot.

We observe from this plot that the AS is bent and the cells present a book-shelving disposition. In addition, maximum cell-to-cell misalignment in the $X$ axis is bigger than the requirements shown in Figure 2.5. This is indicated by the red arrows for both axes. These values are an indication of the maximum tilt present in the structure with respect of the longitudinal axis by fabrication, which is $169.3 \mu \mathrm{rad}$ in the $X$ axis and $40.15 \mu \mathrm{rad}$ in the $Y$ axis. These tilts could be due the deformation produced by the handling, transportation and fixing tool of the AS. So, these positions of the geometric centre are only an estimation of the geometric imperfections of the structure that we use after to measure its EM center.

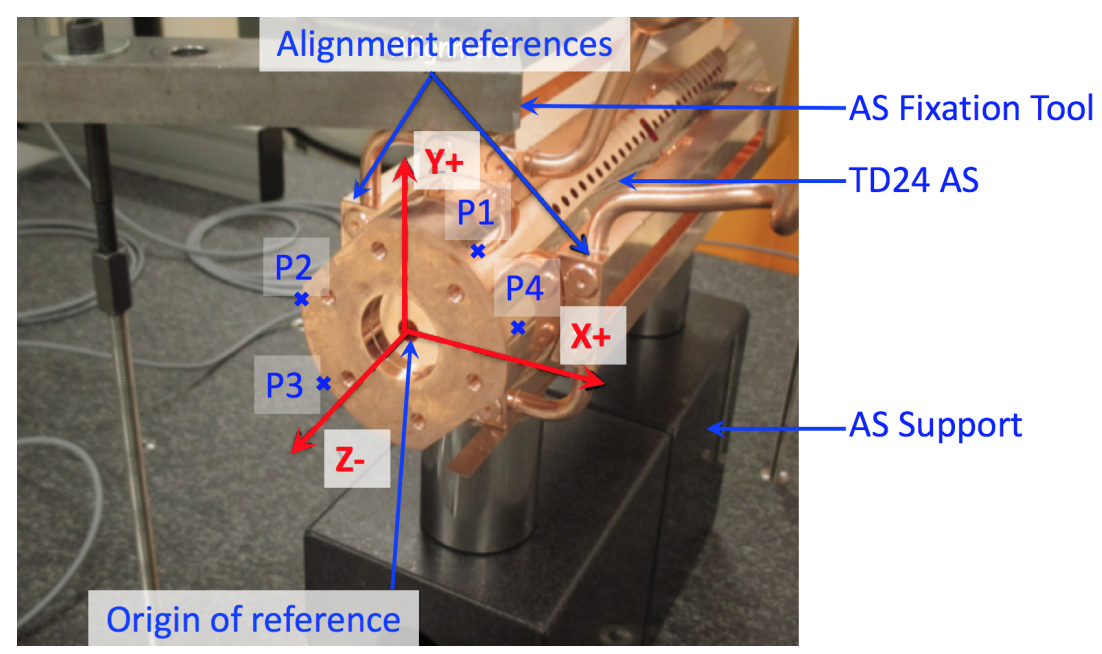

Figure 2.6: Set-up installed in the CMM to measure the outer geometry of the TD24. The origin of reference is the first matching cell with the axes defined as shown by the red arrows. 


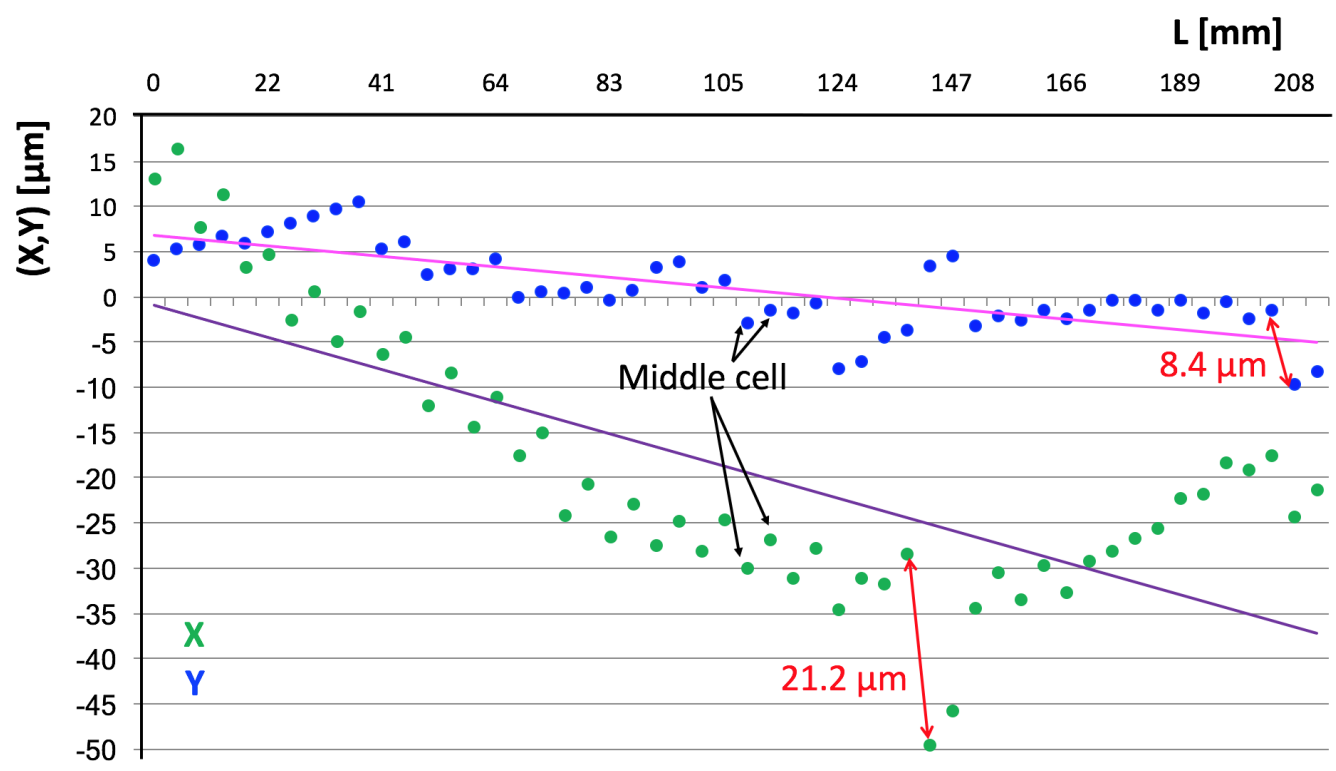

Figure 2.7: Estimated position of the geometric centre in $X$ (green dots) and $Y$ (blue dots) with respect to the length, $L$, of the TD24 using external topographic measurements with the CMM. The purple and pink solid lines represent the linear fit of the geometric centres in each axis $X$ and $Y$, respectively. The maximum cell-to-cell misalignment is indicated by the red arrows.

\subsection{New methodology to measure the EM centre of AS for align- ment purposes}

\subsubsection{Wire as an excitation: the coaxial wire method and the twinax method}

The first stretched wire measurement bench was developed at the Stanford Linear Accelerator Centre (SLAC) in the USA in 1974 to measure the energy loss of a stored beam to a cavity [67]. This development has inspired longitudinal impedance measurements of ASs and other components [68] as well as transverse wakefield measurements in ASs. The closest attempt to our purposes concerns the prediction of transverse wakefields in the Detuned Structure number 2 (DS2) with 206 cells developed for the Next Linear Collider (NLC) [69].

This technique exploits the hypothesis that it is possible to simulate the effect of an electron beam passing through an AS by studying the progress of the TEM field excited in a perfect conducting cylinder in which an electrically excited conductor wire has been inserted [70].

The electric field of an ultra-relativistic charge $q$ moving at the speed of light $c$ along the centre of a beam pipe is written in cylindrical coordinates as:

$$
E_{r}(r, \omega)=Z_{0} H_{\phi}(r, \omega)=\frac{Z_{0} q}{2 \pi r} e^{-j \frac{\omega}{c} z}
$$


where $r$ is the radial distance from the $z$ axis of the charge, $Z_{0}$ is the characteristic impedance of free space and $\omega$ is the angular frequency related to the frequency $f$ as $\omega=2 \pi f$. This equation is the same for an excited TEM like field in a cylindrical waveguide by an impulse of current with total charge $q=I_{0}$ traveling along a centre conductor in the beam pipe, see Figure 2.8.

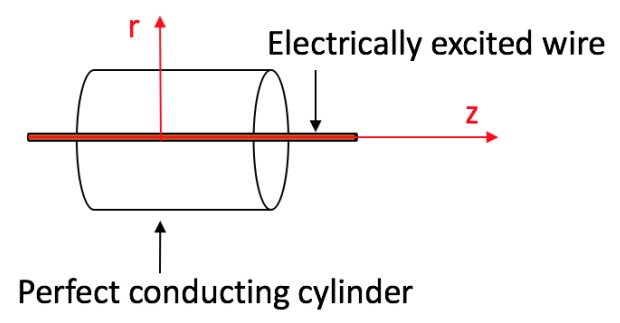

Figure 2.8: Perfect conducting cylinder with centred conductor wire in a cylindrical coordinate system.

When a cylindrical waveguide or beam pipe is connected to a cavity, the physical effect created when exciting the wire with a current pulse will be the same as the wakefield created by a traveling electron bunch, or leading bunch, encountering this geometry variation. As the current pulse reaches the cavity, due to the geometric discontinuity, charges are deposited in the walls and induce a secondary field that will act on the pulse.

The forces exerted by the leading bunch on a trailing electron bunch in the direction of motion of the beam $(z)$ and in the transverse plane $(r)$ can be obtained from Eq (2.2). The detailed expression could be found in [71]. We could obtain the induced longitudinal and transverse wake potentials of a particular mode in cylindrically symmetric structures, $W_{z}(s)$ and $W_{t}(s)$ respectively, as:

$$
\begin{gathered}
W_{z}(s)=2 k_{\text {loss,mode }} \cos \left(\omega_{\text {mode }} s / c\right) \\
W_{t}(s)=\frac{2 k_{\text {loss,mode }} r / a_{0}}{\omega a_{0} / c} \sin \left(\omega_{\text {mode }} s / c\right)
\end{gathered}
$$

where $r$ is the offset of the driving bunch, $a_{0}$ is the position at which the loss factor is calculated, $k_{\text {loss,mode }}$ is the loss factor of the mode and $\omega_{\text {mode }} / 2 \pi$ is the mode frequency [60]. The total longitudinal wake potential is obtained by summing over all the modes, and the same applies for the transverse wake potential.

The objective is the measurement of the loss factor in order to characterise the wakefields induced within a cavity following the formulas (2.3) and (2.4). For this purpose, first, the impedance of the cavity with an inner conductor wire [67] will be characterised. Second, the cavity will be considered well matched at both sides and the power reaching the end of the cavity when the wire is electrically excited at the input of the cavity will be calculated. The formulae is developed in reference [72] and show how this ratio is related to the loss factor. Finally, the wakefields could be obtained.

A cavity near resonance, sketched in Figure 2.9 (a), can be represented by the equivalent RLC circuit shown in Figure 2.9 (b). Each mode of the cavity is characterised by 3 
parameters: $\omega_{0}$ is the natural frequency of the cavity, $r_{0}$ is the intrinsic impedance and $Q_{0}$ is the loss coefficient. The values of the RLC equivalent are determined as a function of such parameters as:

$$
\begin{gathered}
R_{\|}=r_{0} Q_{0} \\
L=\frac{r_{0}}{\omega_{0}} \\
C=\frac{1}{r_{0} \omega_{0}} \\
Q_{0}=R_{\|} \sqrt{\frac{C}{L}}
\end{gathered}
$$

When a wire is inserted in the cavity, a few lumped impedances are added to the initial RLC circuit, as shown in Figure 2.10 (a), and changing the resonant frequency. The new equivalent circuit is shown in Figure 2.10 (b), where $L^{\prime}$ represents the wire self inductance, $C^{\prime}$ is the capacitance formed between the wire and the edges at both sides of the cavity and $R_{0}$ is the characteristic resistance of the line formed by the wire with the vacuum pipe on either side of the cavity. These elements are geometry dependent as:

$$
\begin{gathered}
L^{\prime}=\frac{\mu_{0}}{2 \pi} g \ln \left(\frac{D}{d}\right) \\
C^{\prime}=\frac{2 \pi \varepsilon_{0} D}{\ln \left(\frac{D}{d}\right)} \\
R_{0}=\frac{1}{2 \pi} \sqrt{\frac{\mu_{0}}{\varepsilon_{0}}} \ln \left(\frac{D}{d}\right)=60 \ln \left(\frac{D}{d}\right)
\end{gathered}
$$

where $d$ is the wire radius, $D$ is the pipe radius and $g$ is the cavity length. The change in the resonant frequency $\omega_{0}$ due to the wire, could be approximately expressed as the change that would occur by replacing $L$ of the cavity by the parallel combination of $L$ and $L^{\prime}$. Considering $L^{\prime} \gg L$, we obtain:

$$
\frac{\Delta \omega}{\omega_{0}} \approx \sqrt{\frac{L}{L^{\prime}}}=\frac{\pi r_{0}}{\omega_{0} \mu_{0} g} \frac{1}{\ln \left(\frac{D}{d}\right)}
$$

In a similar way, the change in the quality factor $Q$ is expressed as:

$$
Q \approx \frac{2 R_{0}}{r_{0}}\left(1+\left(\frac{\omega_{0} g}{2 c}\right)^{2}\right)=\frac{120}{r_{0}}\left(1+\left(\frac{\omega_{0} g}{2 c}\right)^{2}\right) \ln \left(\frac{D}{d}\right)
$$

with $c$ the speed of light [67].

A simplification of the previous circuit model could be applied given that an AS with an inner metallic wire as sketched in Figure 2.11 (a), looks physically as a coaxial wire. This gives the name to the coaxial wire method. Coaxial cables are composed of two concentric conductors, maintained at a fixed spacing by a dielectric separator. Equivalently, the outer conductor of the coaxial wire is the copper walls of the AS, with a radius equal to the iris; the dielectric medium is the air inside the AS; and the inner conductor is the wire. This is indicated as blue and black labels in Figure 2.11 (a). The equivalent circuit of a coaxial wire is shown in Figure 2.11 (b). The elements of the previous equiv- 
alent circuit of a resonant cavity with an inner conductor wire could be reorganised and the equivalent circuit of a coaxial wire could be obtained. A cylindrical coaxial wire is characterised by the capacitance $C^{\prime \prime}$ between the two conductors, its inductance $L^{\prime \prime}$, the resistance $R$ of the conductors and the conductance $G$ of the dielectric material between the two conductors. They can be calculated as:

$$
\begin{gathered}
C^{\prime \prime}=\frac{2 \pi \varepsilon_{0} \varepsilon_{r}}{\ln \left(\frac{D}{d}\right)} \\
L^{\prime \prime}=\frac{\mu_{0} \mu_{r}}{2 \pi} \ln \left(\frac{D}{d}\right) \\
R^{\prime \prime}=\rho \frac{l}{A} \\
G^{\prime \prime}=\frac{A}{\rho l}
\end{gathered}
$$

where $D$ is the diameter of the outer conductor, $d$ is the diameter of the inner conductor, $\varepsilon_{r}$ is the relative permeability of the medium, $\varepsilon_{0}$ is the electric constant of free space, $\mu_{r}$ is the relative permeability, $\mu_{0}$ is the permeability of free space, $\rho$ is the electrical resistivity of the material, $A$ is the cross-section area of the conductor and $l$ is the length of the conductor. At high frequencies, $R^{\prime \prime}$ and $G^{\prime \prime}$ become less important relative to $L^{\prime \prime}$ and $C^{\prime \prime}$ and the characteristic impedance $Z^{\prime \prime}$ becomes:

$$
Z^{\prime \prime}=\sqrt{\frac{R^{\prime \prime}+j \omega L^{\prime \prime}}{G^{\prime \prime}+j \omega C^{\prime \prime}}} \approx \sqrt{\frac{L^{\prime \prime}}{C^{\prime \prime}}}=\frac{1}{2 \pi} \sqrt{\frac{\mu_{0}}{\varepsilon_{0} \varepsilon_{r}}} \ln \left(\frac{D}{d}\right) \approx \frac{60}{\sqrt{\varepsilon_{r}}} \ln \left(\frac{D}{d}\right)[\Omega]
$$

The impedance of the cavity $Z^{\prime \prime}$ is considered to be perfectly matched to a source $V$ with impedance $Z_{g}=Z_{0}$ and to an output load $Z_{L}=Z_{0}$, as shown in Figure 2.11 (c). A bench setup consisting on a VNA acting as the source $V$ with input and output impedances $Z_{0}$, is connected at both sides of the cavity [69] [72] and is used to measure the transmitted power $P_{0}$ to the output impedance $Z_{0}$ with respect to the power dissipated in the cavity $P_{\text {diss. }}$. This is given by:

$$
\frac{P_{\text {diss }}}{P_{0}}=1-T
$$

where $T$ is the transmission coefficient at the output $Z_{0}$, and can also be expressed as:

$$
T=\left|S_{21}\right|^{2}=\frac{Z_{0}}{2 P_{0}}|I|^{2}
$$

where the current $I$ is:

$$
I=\frac{V}{2 Z_{0}+Z^{\prime \prime}}
$$

As $P_{0}$ is:

$$
P_{0}=\frac{V^{2}}{8 Z_{0}}
$$


Then, substituting $P_{0}$ and $I$ in Eq (2.20) and rewriting $Z^{\prime \prime}$ as $Z^{\prime \prime}=R_{e}+j X_{i m}$ being $R_{e}$ and $X_{i m}$ the real and imaginary terms of $Z^{\prime \prime}$ respectively, the transmission coefficient is obtained as:

$$
T=\frac{4 Z_{0}^{2}}{\left(2 Z_{0}+R_{e}\right)^{2}+X_{i m}^{2}}
$$

At the resonant frequency, $T$ becomes:

$$
T_{0}=\frac{4 Z_{0}^{2}}{\left(2 Z_{0}+R^{\prime \prime}\right)^{2}}
$$

The power dissipated in the cavity is, therefore:

$$
1-T=\frac{R_{e}^{2}+4 Z_{0} R_{e}+X_{i m}^{2}}{\left(R_{e}+2 Z_{0}\right)^{2}+X_{i m}^{2}}
$$

$1-T$ could be measured with respect to the frequency, using a VNA and the result shown in Figure 2.12 could be obtained. The minimum value of $T$ observed at the resonant frequency $f_{0}$ corresponds to $T_{0}$. The area $A$ of width $\Delta f_{1 / 2}$ under the curve 1-T gives the power dissipated, like:

$$
A=\frac{1}{\pi} \int_{-\infty}^{\infty}(1-T) d \omega=\frac{\omega_{0} R^{\prime \prime}}{8 Z_{0} Q}\left(\frac{R^{\prime \prime}+4 Z_{0}}{R^{\prime \prime}+2 Z_{0}}\right)
$$

On the other hand, the loss factor is given in the RLC parallel by:

$$
k_{\text {loss }}=\frac{\omega_{0} R^{\prime \prime}}{2 Q}
$$

The term in brakets in Eq (2.26) can be replaced by $1+\sqrt{T_{0}}$ in Eq (2.24). Substituting $\mathrm{Eq}(2.26)$ in the $k_{\text {loss }}$ formula in Eq (2.27) we obtain:

$$
k_{\text {loss }}=\frac{4 Z_{0}}{1+\sqrt{T_{0}}} A
$$

Since the $1-T$ function is Lorentzian, we can rewrite it as:

$$
1-T=\frac{1-T_{0}}{1+\left(2 \frac{f-f_{0}}{\Delta f_{1 / 2}}\right)^{2}}
$$

where the area is obtained from the width of the function as:

$$
A=\frac{\pi}{2} \Delta f_{1 / 2}\left(1-T_{0}\right)
$$

And we end up with a loss factor formula:

$$
k_{\text {loss }}=2 \pi Z_{0} \Delta f_{1 / 2}\left(1-T_{0}\right)
$$




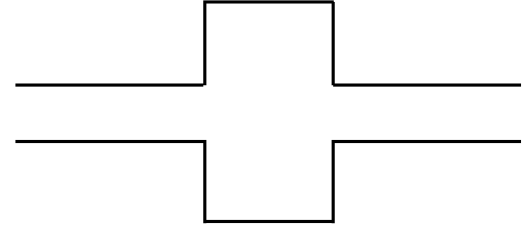

(a)

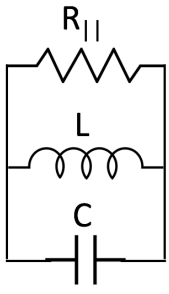

(b)

Figure 2.9: (a) Sketch of a resonant cavity and (b) equivalent RLC circuit.

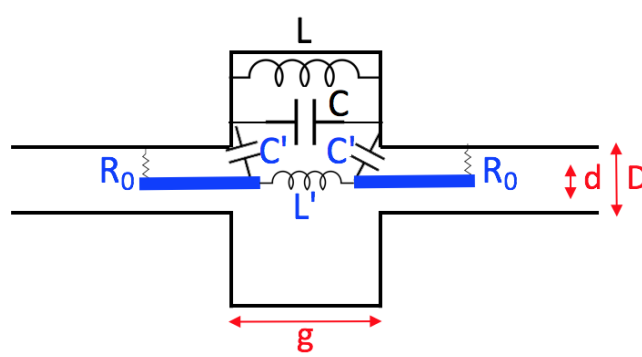

(a)

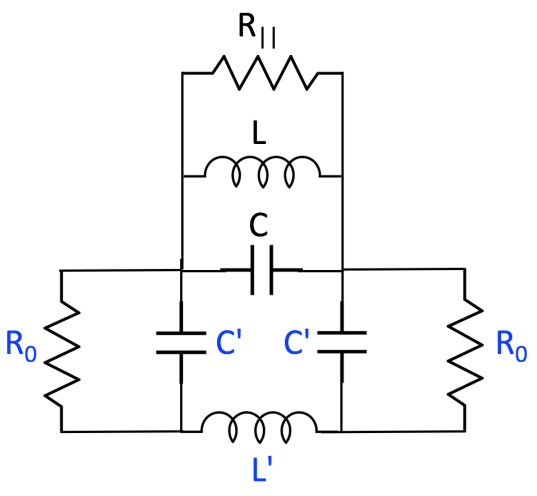

(b)

Figure 2.10: (a) Sketch of a resonant cavity with a conductor wire and (b) equivalent circuit at high frequency.

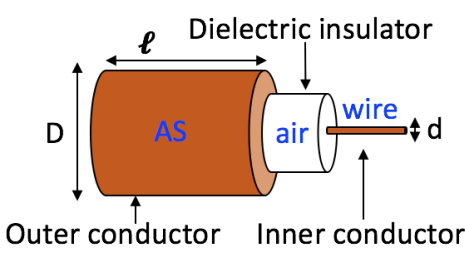

(a)

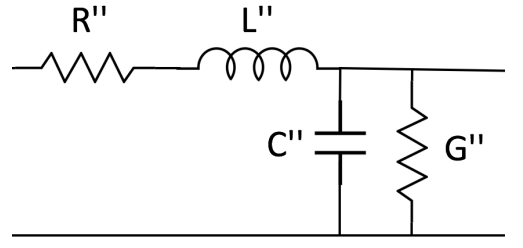

(b)

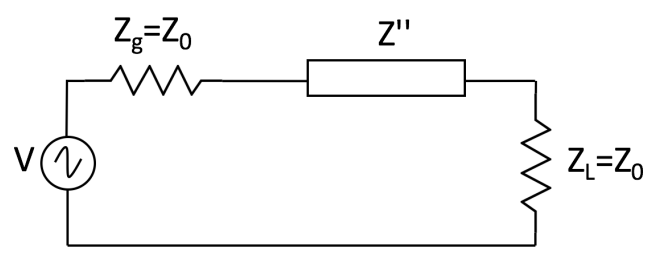

(c)

Figure 2.11: (a) Coaxial wire configuration with critical dimensions, (b) equivalent circuit and (c) characteristic impedance $Z^{\prime \prime}$ in a circuit with matched input $Z_{g}$ and matched output $Z_{L}$. 


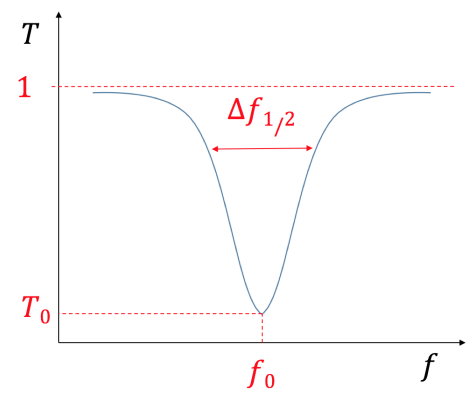

Figure 2.12: Power transmitted from the input of the AS to the output. The resonant mode is identified as a minimum.

Therefore, the only necessary measurement to be performed in order to obtain the loss factor of each mode coupling to the wire is a transmission measurement $T$ using a VNA. For each mode and each transverse wire position, $T_{0}$ is obtained at its corresponding frequency $f_{0}$ and also the half power points $\Delta f_{1 / 2}$. The amplitude of the peaks change with the wire position and also a frequency shift is expected. Simulation studies using the NLC structures in SLAC have determined that the frequency $f_{0}$ in each cell, that is modelled as a resonant cavity, coincides to the frequency of the unperturbed dipole when the wire approaches the limit of the EM centre. As a consequence, the expected value of the loss factor is equal to the one without the wire [73].

We studied the feasibility of the coaxial wire technique in the particular case of the TD24, whose configuration can be seen in Figure 2.13. The measurement was foreseen to be performed through the available RF coaxial pick-ups of the WFM that are coupled to the damping waveguides in the middle cell, see Figure 2.1. Each WFM of the TD24 has two RF pick-ups, one that couples to a beam-excited dipole mode at $18 \mathrm{GHz}$ and another one that couples a beam-excited monopole mode at $24 \mathrm{GHz}$. We aim to connect the four RF pick-ups that couple to the dipole mode to four power meters. We should terminate the other RF-pick ups with a load of $50 \Omega$ in order to have a proper match in the system.

Since the TD24 is not a resonant cavity and all frequencies are supported, we cannot measure the loss factor of each resonant mode like in a resonant cavity. A solution based in the combination of the power signals reaching the WFMs is investigated. The power signals reaching the RF pick-ups in each plane will be combined independently in the transversal axes, $X$ and $Y$, at each wire position in such a way that they follow a V-shape relationship as shown in Figure 2.14. The dip of the plot represents the position of the EM centre, so the goal would be to obtain the wire position where the combined signals have a minimum.

Several elements were identified for the manufacturing of the measurement set-up: a signal launcher to be connected at the first coupling cell of the AS, two beam pipes with bellows to be plugged at both extremes of the AS, a matching circuit to be connected at the last cell of the structure, and an electronic acquisition and processing in order to combine the signals reaching the WFM. Due to the heavily damped AS, we expect that the power level of the signals reaching the WFM will be very low. Most of the power down the wire would be absorbed in the first damping waveguides, so the measurements are expected to 


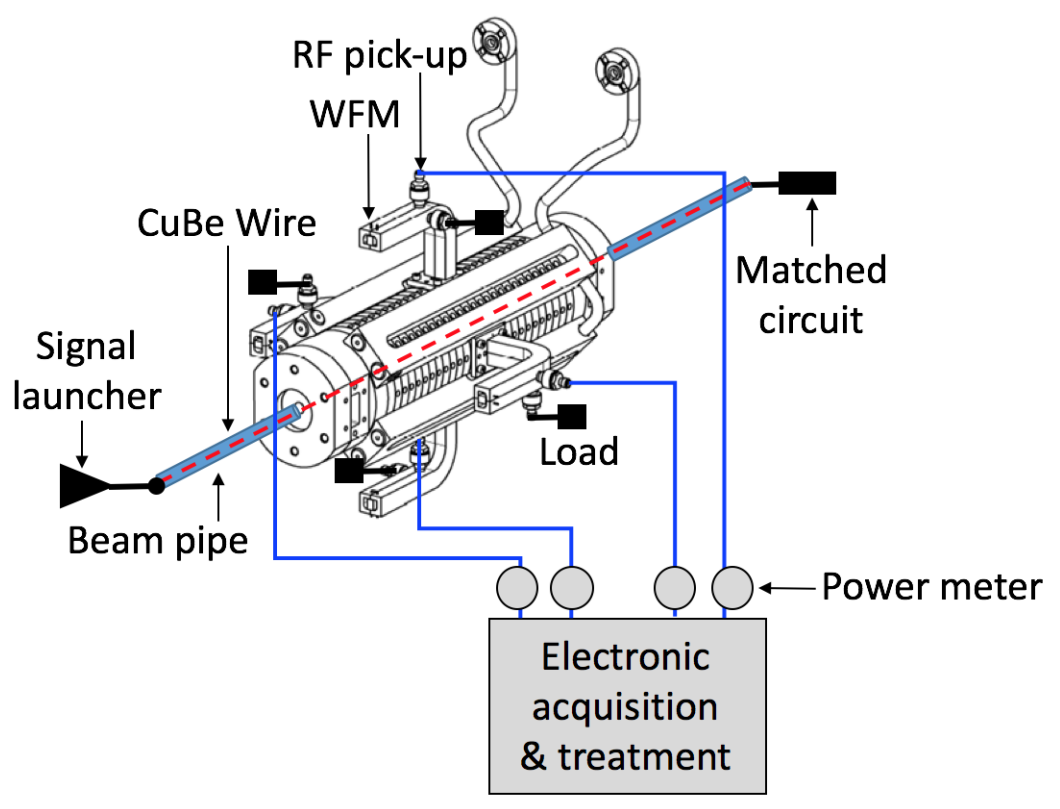

Figure 2.13: The coaxial wire method using the TD24.

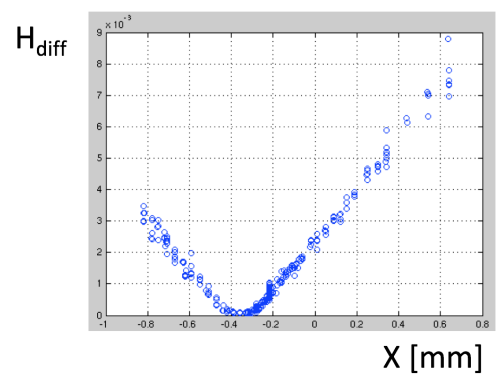

Figure 2.14: Combined power signals $H_{\text {diff }}$ reaching the WFM in the $X$ axis for different wire positions in $X$.

be performed with a very poor resolution. Therefore, the coaxial wire method is not a feasible solution and a different method is studied to avoid this.

An alternative is to concentrate the power between two parallel cylindrical conductors of radius $d$ separated by a distance $s$ from each other, similar to a balanced transmission line, see Figure 2.15. This could be achieved establishing one wire at a potential $V / 2$ with an excitation current $I$, and setting the other wire at a potential $-V / 2$ with a current $-I$. This configuration is routinely used for estimating transverse impedance of ASs [74]. Following a similar argument rather than the one-wire, the characteristic impedance of the balanced two-wire line inside a circular conducting tube $Z^{\prime \prime \prime}$ is:

$$
Z^{\prime \prime \prime}=\frac{276 \log _{10}\left(\frac{2 s}{D}\right)}{\sqrt{\varepsilon_{r}}}\left[\frac{1-(L / D)^{2}}{1+(L / D)^{2}}\right]
$$

being $L$ centre to centre inner conductor spacing. 


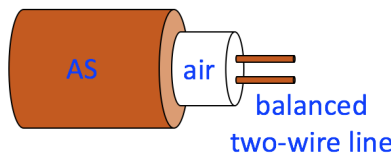

(a)

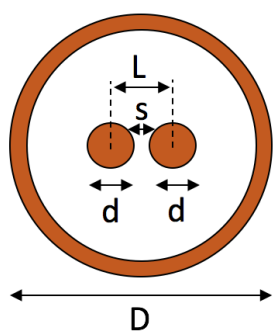

(b)

Figure 2.15: (a) Balanced transmission line and (b) cross section with critical dimensions.

The so-called twinax mode created with the two excited wires is such that the EM field around the conductors is equal in magnitude and opposite in phase, and couples to the dipole modes of the structure. The AS is moved at different transverse offsets with respect to the EM centre, and the same conceptual design can be applied to measure the power signals reaching the WFM. However, a mechanical challenge involves the manufacturing of the two perpendicular wires with a distance $L$ small enough with respect to the small irises of the AS. In addition to that, the design and manufacturing of critical components, like the beam pipes and the matching circuit suited for this application, is rather difficult. Furthermore, the beam pipes requires low discontinuities in their coupling within the small irises of the TD24. Likewise, the matching circuit requires the manufacturing of a wire with variable radius in order to avoid reflections given the variable impedance along the tapered TD24. In addition to that, a technique using two parallel wires is not a suitable solution within the PACMAN project: the solutions developed by the network concerns the determination of the absolute position of an accessible single wire made of copper beryllium with a diameter of $100 \mu \mathrm{m}$. Moreover, the visibility of the wire would be lost with the installation of the beam pipes. Due to all these difficulties, the coaxial wire method with one or two wires was discarded for implementation and the feasibility of a new technique was studied.

\subsubsection{The new technique: the perturbative method}

As the method proposed before is not easily implemented in this kind of AS, we propose a new technique similar to the bead-pull measurements that is typically used for AS tuning [75] [76] and HOM measurements [77]. The bead-pull technique exploits the Slater's perturbation theorem in order to characterise the field distribution in a resonant EM cavity [60].

The theorem states that in a cavity on resonance where the electric and magnetic stored energies are equal, when a perturbation is introduced, it unbalances both energies, leading to a shift on the resonance frequency $\omega_{0}$ to balance again the stored energies. For small perturbations, the frequency shift $\Delta \omega_{0}$ is proportional to the original amount of energy stored in the perturbing object, and the stored energy is proportional to the square of the EM fields. Therefore, the frequency shift depends on the local EM fields at the position of the perturbing object. This way, the EM fields in a resonant cavity could be obtained by measuring the change in the resonance frequency caused by a small perturbation 
in the cavity following the next equation:

$$
\frac{\Delta \omega_{0}}{\omega_{0}}=\frac{\int_{\Delta V}\left(\mu_{0} H^{2}-\varepsilon_{0} E^{2}\right) d V}{\int_{V}\left(\mu_{0} H^{2}+\varepsilon_{0} E^{2}\right) d V}=\frac{\Delta U_{m}-\Delta U_{e}}{U}
$$

being $\Delta V$ the volume of the perturbation that is removed from the cavity of volume $V, E$ and $H$ are the unperturbed electric and magnetic field amplitudes respectively, $U$ is the total stored energy, $\Delta U_{m}$ is the time average of the stored magnetic energy removed and $\Delta U_{e}$ is the time average of the stored electric energy removed as a result of the reduced volume. $U, U_{m}$ and $U_{e}$ can be expressed as a function of the unperturbed fields as:

$$
\begin{gathered}
U=\frac{1}{4} \int_{V}\left(\mu_{0} H^{2}+\varepsilon_{0} E^{2}\right) d V \\
\Delta U_{m}=\frac{1}{4} \int_{\Delta V}\left(\mu_{0} H^{2}\right) d V \\
\Delta U_{e}=\frac{1}{4} \int_{\Delta V}\left(\varepsilon_{0} E^{2}\right) d V
\end{gathered}
$$

In the bead-pull technique, the perturbation is introduced in the cavity by pulling a small dielectric or metallic object, known as bead, suspended by a thread that is moved through the cavity. As the bead is pulled along the AS, the resonance frequency is monitored as a function of the position of the bead. The measurement determines the ratio of squared field to the stored energy. The stored energy can be determined from Eq (2.37) and from separate measurements of $Q$ and the cavity power dissipation $P$, as:

$$
U=\frac{Q P}{\omega_{0}}
$$

Two types of perturbations could be applied: a dielectric and a metallic bead with a volume small compared to the one of the cavity. Besides, three bead geometries can be used: spheres, disks and needles. They all have different effects in the EM fields, that also depends on their orientation and size. For instance, in the particular case of using a spherical bead of volume $\Delta V$, the shift is given by:

$$
\frac{\Delta \omega_{0}}{\omega_{0}}=-\frac{3}{4} \frac{\Delta V}{U}\left[\frac{\mu_{r}-1}{\mu_{r}+2} \mu_{0} H^{2}+\frac{\varepsilon_{r}-1}{\varepsilon_{r}+2} \varepsilon_{0} E^{2}\right]
$$

For a spherical dielectric bead: $\mu_{r}=1$, and:

$$
\frac{\Delta \omega_{0}}{\omega_{0}}=-\frac{3}{4} \frac{\Delta V}{U} \frac{\varepsilon_{r}-1}{\varepsilon_{r}+2} \varepsilon_{0} E^{2}
$$

as Eq (2.39) shows, the electric field in a resonant cavity can be characterised by measuring the frequency shift created by a perturbing dielectric bead.

For a spherical metallic bead: $\varepsilon_{r} \rightarrow-j \infty$ and $\mu_{r} \rightarrow 0$, Eq (2.38) becomes:

$$
\frac{\Delta \omega_{0}}{\omega_{0}}=-\frac{3}{4} \frac{\Delta V}{U}\left[-\frac{\mu_{0} H^{2}}{2}+\varepsilon_{0} E^{2}\right]
$$


We can see from Eq (2.40) that both electric and magnetic fields in a cavity can be obtained by measuring the frequency shift created by a perturbing metallic bead. So, in order to characterise the whole EM field in a cavity, pulling both dielectric and metallic beads are necessary.

In the case of using a needle shape of volume $\Delta V$, the needle perturbs strongly the electric fields aligned with it and perturbs weakly the perpendicular electric and magnetic fields [78], expressed as:

$$
\frac{\Delta \omega_{0}}{\omega_{0}}=\alpha_{E \|} \varepsilon_{0} \frac{E_{\|}^{2}}{U}+\alpha_{E \perp} \varepsilon_{0} \frac{E_{\perp}^{2}}{U}+\alpha_{H \perp} \mu_{0} \frac{H_{\perp}^{2}}{U}
$$

where $E_{\perp}$ and $H_{\perp}$ are the transverse electric and magnetic fields, respectively, $E_{\|}$is the longitudinal electric field and $\alpha_{E \|}$ is the approximated longitudinal factor and $\alpha_{E \perp}$ and $\alpha_{H \perp}$ are the approximated transversal factors. $\alpha_{E \|}$ is calculated as:

$$
\alpha_{E \|}=\pi \frac{l^{3}}{24(\log (l / r)-1)}
$$

being $l$ the length of the needle and $r$ the radius of the needle.

The new method that we propose in this thesis is inspired in the bead-pull technique applied in the tuning of the $\mathbf{X}$-band traveling-wave AS at CERN [76]. In travelingwave structures, like the TD24, frequency de-tuning in each cell cause reflection from the output matching cell that may lead to the limitation of the overall accelerating gradient. The technique considers an input and output port in each cell as well as the previous cell and the next cell, respectively. The reflection $\Gamma$ from the input port that couples to a traveling-wave structure at the working frequency is approximated considering perfect coupling as:

$$
\Gamma \approx j \frac{c \varphi}{v_{g}} \frac{\Delta \omega_{0}}{\omega_{0}}
$$

where $\Delta \omega_{0}$ is the detuning of the cell, $\varphi$ is the phase advance per cell and $v_{g}$ is the group velocity of the structure. Eq (2.43) indicates that the reflection of each cell can be corrected through a local frequency change in order to make $\Gamma=0$. In order to apply this, the tuning method is performed in several steps using a VNA connected to the input matching cell of an AS and two matching loads connected to the output cell, as shown in Figure 2.16 (a). First, the electric field distribution is obtained along the structure by pulling a small dielectric spheric bead. Second, the reflection in each cell is obtained by calculating the forward wave $A_{n}$ and backward wave $B_{n}$ along the structure in each cell, see Figure 2.16 (b), that are given by:

$$
A_{n}=\frac{I_{n-1}-I_{n} e^{-j \varphi}}{2 j \sin \varphi}, B_{n}=\frac{I_{n-1}-I_{n} e^{j \varphi}}{-2 j \sin \varphi}
$$

where $I_{n}$ is the field of the cell $n$. The reflection local parameter $\Gamma_{\text {local } n}$ for a given cell $n$ except for the first and the last cells, is expressed as:

$$
\Gamma_{\text {local } n}=\frac{B_{n}-B_{n+1} e^{-j \varphi}}{A_{n}},(2 \leq n \leq N-1)=\left.S_{11}^{\text {local }}\right|_{n}
$$


The output matching cell $n=N$ is connected to a matched load, so no reflection is expected. The reflection $\Gamma_{\text {local } N}$ for this particular cell is given by:

$$
\Gamma_{\text {local } \mid N}=\frac{B_{N}}{A_{N}}=\left.S_{11}^{\text {local }}\right|_{N}
$$

In a similar way, the reflection $\Gamma_{\text {local } 1}$ in the input matching cell, with $n=1$, is related to the reflection measured by the VNA at the input cell $S_{11}$ following:

$$
\Gamma_{\text {local } 1}=\frac{\left[-j\left|\Delta S_{11}\right| / \Delta S_{11}\right] S_{11} A_{1}-B_{2} e^{-j \varphi}}{A_{1}}=\left.S_{11}^{\text {local }}\right|_{1}
$$

where the measured reflection coefficient of the whole structure is $S_{11}$ and the change of the reflection $\Delta S_{11}$ caused by the introduction of the bead into the first cell, $n=1$, are:

$$
\begin{gathered}
S_{11}=e^{-2 \varphi_{0}} \frac{B_{1}}{A_{1}} \\
\Delta S_{11}=e^{-2 j \varphi_{0}}\left(-j\left|\Delta S_{11}\right|\right)
\end{gathered}
$$

with $\varphi_{0}$ the phase offset of the input waveguide. When a bead is located in cell $n$ and a local reflection is applied to that particular cell, this local change in the reflection, $\left.\Delta S_{11}^{\text {local }}\right|_{n}$ or $\Delta \Gamma_{\text {local } n}$, could be obtained by measuring the change of the global reflection, $\left|\Delta \Gamma_{\text {global }}\right|$ or $\left|\Delta S_{11}^{\text {global }}\right|$, in the whole structure at the input coupler following:

$$
\left|\Delta \Gamma_{\text {global }}\right|=e^{\alpha}\left|\Delta \Gamma_{\text {local } n}\right|=\left|\Delta S_{11}^{\text {global }}\right|=e^{\alpha}\left|\Delta S_{11}^{\text {local }}\right|_{n}
$$

where $\alpha$ is the attenuation caused in the reflected wave due to the power loss on the cell wall, that is expressed as:

$$
\alpha=-\sum_{m=1}^{n-1} \frac{c \varphi}{Q_{0}(m) v_{g}(m)}
$$

Finally, tuning studs are used to modify the frequency cell-by-cell. The local reflection correction that is applied in this step, is obtained by monitoring the global reflection $\left|\Delta S_{11}\right|$ with the VNA until the target calculated with Eq (2.50) and (2.51) is reached.

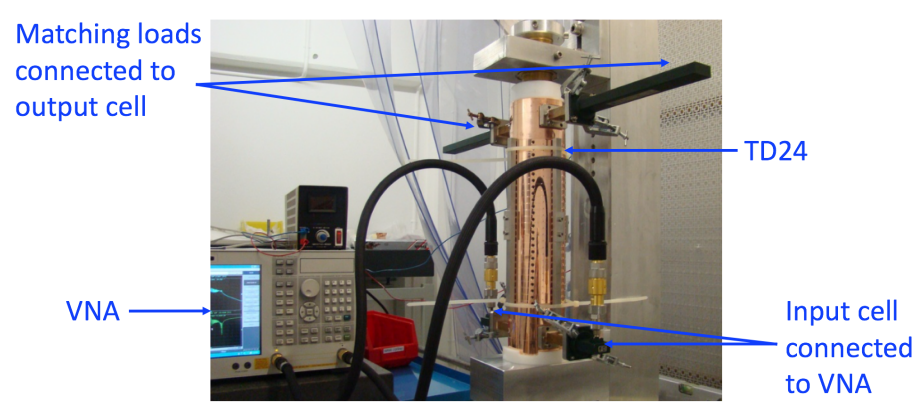

(a)

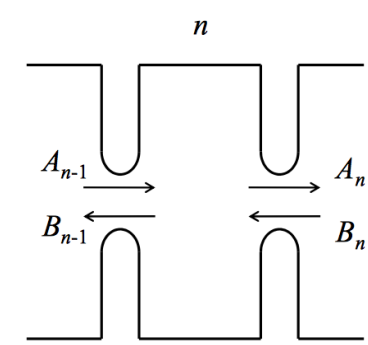

(b)

Figure 2.16: (a) Test bench for AS tuning with VNA ports connected to the input cell and matching loads connected to the output cell. (b) Forward and backward waves $A$ and $B$, respectively at cell $n$ of a traveling-wave structure [76]. 
Similarly to the bead-pull method, we could use a wire inserted along the TD24 as needle perturbing the EM fields. In this case, instead of pulling a bead along the whole length of the TD24, we will fix the wire and move the middle cell in its transverse horizontal and vertical axes, and measure the perturbation of the EM fields created by the wire in the middle cell. As shown in Figure 2.17, we will feed the middle cell through the damping waveguides with power signals. The frequency shift caused by the perturbing metallic PACMAN wire can be obtained from Eq (2.41), taking into account that the small wire radius with respect to its length will cancel the transversal components and, therefore, the wire couples only to the longitudinal field component.

As the cells of the TD24 are not resonant cavities, we can not use the shift in the resonant frequency to sample the EM fields. We also have to take into account that the cross section of the middle cell of the TD24 has a complicated symmetric geometry as shown in Figure 2.3 (a). However, we expect that the wire will perturb the EM fields inside the TD24. If so, it will be possible to monitor the EM fields in the middle cell in all frequencies starting from the cut-off frequency of the damping waveguides, at different transversal wire positions. This way, it could be possible to find the relationship between wire position and frequency shift by exploiting the symmetry of the cell, and we could establish how far the wire is from the EM centre position. The EM fields could be obtained with the combination of a full $4 \times 4$ Scattering $(S)$ parameter matrix measured at the end of the damping waveguides when moving the wire in the horizontal $X$ and vertical $Y$ axes independently. This way, an S-matrix $S_{\text {out,in }}$ could be obtained at each wire position, and we could be able to measure the change on the $S$ parameters as a function of frequency and wire position. Focusing on one single frequency, we could be able to observe a dependance between the combined $S$ parameters in one axis with respect to the wire position as seen in Figure 2.14. Thus, we hypothesise that the wire will preserve the symmetry of the cell and that the perturbation will be minimised at the position of the EM centre. In order to verify this method within the geometrical complexity of the TD24 AS, we need to perform EM field simulations in order to obtain a reliable relationship of the $S$ parameters with respect to the wire position. For this reason, a complete simulation study has been made in Section 2.4.

This new technique is far more preferable for implementation in this case than using an excited wire for different reasons. The first one lies in the simplicity of the instrument equipment needed to perform the measurement consisting only in one four-port VNA. Furthermore, the manufacture of the previous critical elements is no longer necessary. Since the wire is not excited, a signal launcher, a matching circuit and beam pipes are not required. However, the WFM that are clamped to the TD24 adds an asymmetry on the middle cell. This could be solved with the development of waveguide tapered transitions, keeping the symmetry of the cell and avoiding reflections. They could be assembled to the middle cell on one side, while the other one could be connected to the VNA. Another advantage that this technique offers is that the measurement of the EM axis of any AS can be performed in a relatively inexpensive way. As far as we know, this is the first attempt a perturbative method has been used for such application. 


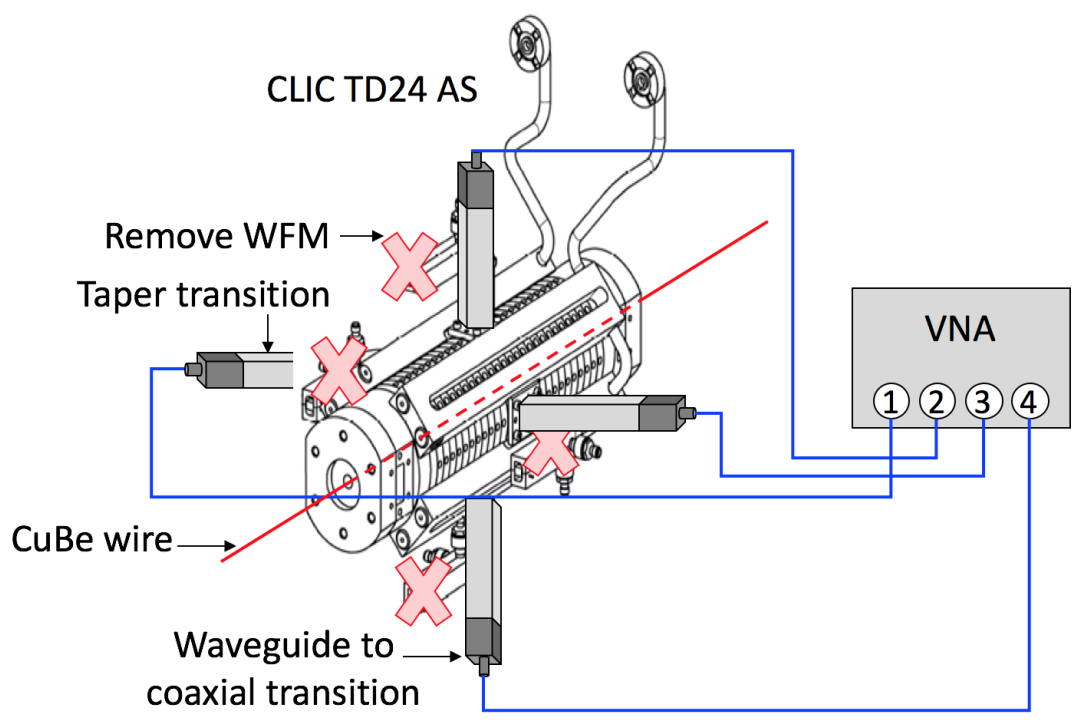

Figure 2.17: The perturbation wire method using the TD24.

\subsection{Experimental proposal to measure the EM axes of the CLIC TD24 AS}

The implementation of the new measurement technique was performed in several steps:

1. The first objective was to determine the feasibility of the new method within the target accuracy of $10 \mu \mathrm{m}$ using the PACMAN wire. This study was performed using an EM field solver. The EM fields in the TD24 AS with and without a perturbing wire were simulated and compared. Later, the wire was moved separately in $X$ and $Y$ and the $S$ parameters were obtained in the middle cell. The relationships between the $S$ parameters delivering the position of the EM centre with respect to the wire position were found at the most sensitive frequency. The simulation results were used for the conceptual design of the measurement algorithm, and also to determine the specifications of an experimental test bench measuring reliably the EM axis in the TD24 AS. Several imperfections on the structure were also simulated in order to study their effect on the detection of the EM centre in a realistic laboratory environment. Finally, as complementary studies, the correlation between the simulated $S$ parameters and the wire parameters, material and dimensions, was also studied looking for maximum sensitivity. This step is developed in the next section.

2. Once the simulation studies demonstrated the feasibility of the method, an existing test bench [79] was adapted for its validation. Several necessary elements of the bench were designed, procured and characterised using microwave measurements. The assembly and calibrations of the test bench were done in the metrology laboratory at CERN in order to minimise the sources of errors previously characterised with simulations. The conceptual design of the measurement algorithm was implemented in a user platform for the automatisation of the measurements. These developments are subject of Chapter 3 . 
3. After that, the measurement process of the EM centre of the TD24 at a controlled temperature could take place in the test bench, and the results obtained were compared with simulations. The repeatability of the new technique was obtained in the metrology laboratory at CERN using position measurement instruments. The results are presented in Chapter 4.

4. Finally, further developments were also investigated using this new technique. For instance, the method could be extrapolated to a test bench developed within this thesis where the AS is positioned horizontally, like it will be in a real accelerator. This way, the absolute wire position could be determined with the solutions developed at PACMAN, and the position of the AS could be reproduced in its girder with fiducialisation methods. The new technique could also be useful to determine the average EM centre of the whole structure. A solution to measure the EM centre of each cell was also investigated in order to verify the internal alignment tolerances during the manufacturing process of AS, before applying bonding.

\subsection{EM field simulation studies to find the EM axes of the CLIC TD24 AS with the PACMAN wire}

EM field simulation studies have been performed using the commercially available code High Frequency Structure Simulator (HFSS) [80]. HFSS is a three-dimension full-wave EM field solver based on finite elements, integral equation and advanced hybrid methods and automatically generates a suitable, efficient and accurate mesh. We serve from this tool to localise the EM centre of the TD24 using the wire as a perturbation technique within the required accuracy of $10 \mu \mathrm{m}$.

The simulations are focused on the behaviour of one single cell, in this case the middle cell, of the TD24 without and with the presence of a conductor wire. The wire to be used within the PACMAN project is made of $98 \%$ of copper and $2 \%$ of beryllium and has a radius of $50 \mu \mathrm{m}$. Therefore, for simplicity, a pure copper wire will be used in the following simulation studies. On the other hand, the transmitted and reflected power signals between the ports defined in the middle cell are obtained in the $\mathbf{S}$ parameter form. The four-port distribution is shown in Figure 2.18 and are represented as black lines, where ports 1 and 3 are facing each other in the $X$ axis and ports 2 and 4 are facing each other in $Y$. In the next subsections, the combination of the simulated $S$ parameters providing the position of the EM centre are presented.

\subsubsection{EM fields and $S$ parameters in the middle cell with and without a perturbing wire}

In this subsection, we are concerned about simulating the EM field distribution only in the middle cell of the AS with and without the copper wire. For this purpose, we simulated the $S$ parameters doing a frequency sweep from $16.5 \mathrm{GHz}$ to $18.5 \mathrm{GHz}$ with steps of $0.01 \mathrm{GHz}$, and obtained the EM field distribution when exciting from one port. 
The resulting EM fields when exciting the cell from port 1 (p1) are presented in Figure 2.18. As observed, the fields are higher next to the input port, most probably due to the multiple reflections in the closest walls. So, the direct transmission from p1 and p3 is asymmetric. Another observation is that the transmission between p1 and the adjacent ports 2 and 4 is symmetric.
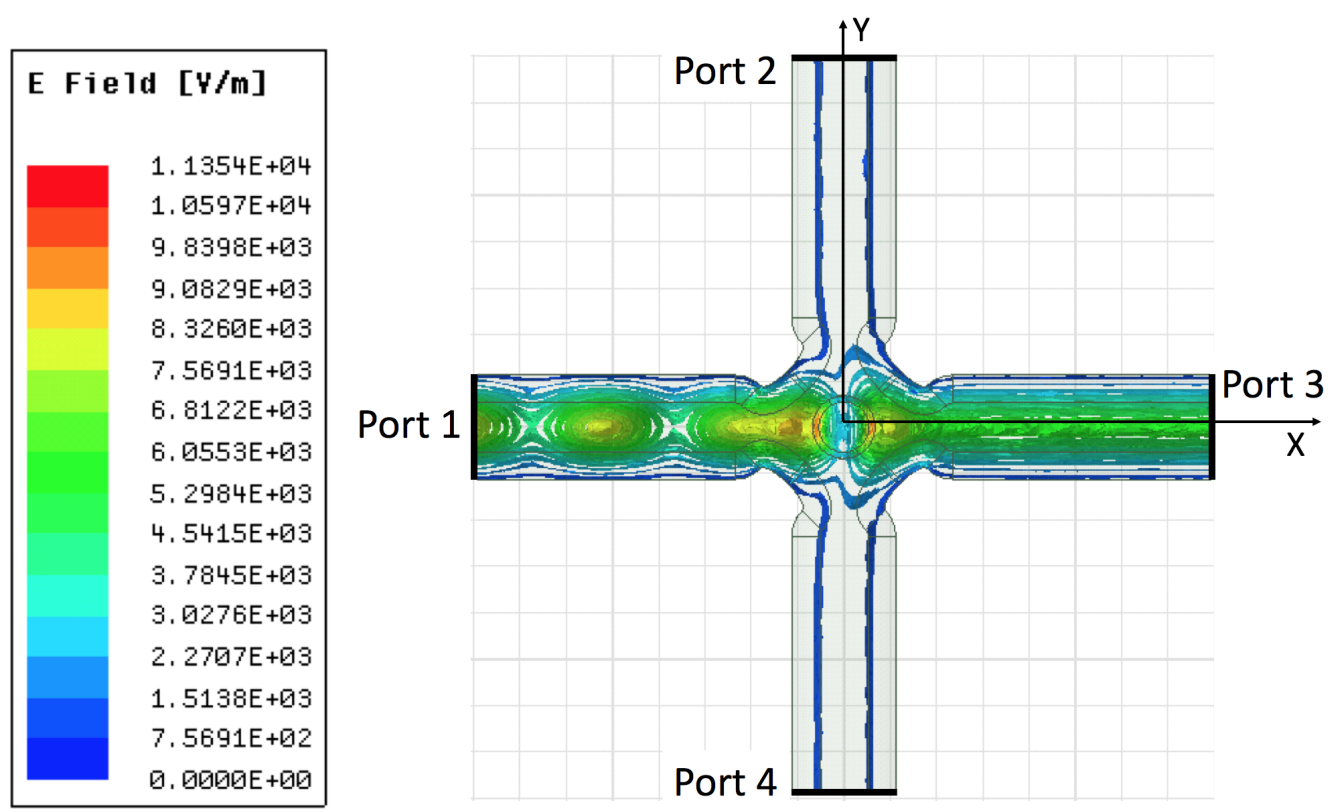

Figure 2.18: Representation of the EM field lines of the unperturbed middle cell (cell 13) of the TD24 AS. In the picture, the input port has been defined at port 1.

The $S$ parameters describe the input-output relationship between ports in an electrical system and are typically expressed as $S_{i j}$, where $i$ refers to the output port and $j$ to the input port. Reflection is represented as $i=j$ and transmission is described as $i \neq j$. In our particular case, when the cell is excited from the input $\mathrm{p} 1$, the power reflected power at p1 is denoted as $\left|S_{11}\right|^{2}$ and the transmitted power to ports 2,3 and 4 are $\left|S_{21}\right|^{2},\left|S_{31}\right|^{2}$ and $\left|S_{41}\right|^{2}$, respectively. They can be represented in linear form or logarithmic form, where the relationship is given by:

$$
S_{i j}[\mathrm{~dB}]=10 \log \left(\left|S_{i j}\right|^{2}\right)[\mathrm{dB}]=20 \log \left(\left|S_{i j}\right|\right)[\mathrm{dB}]
$$

or equivalently in the linear form:

$$
\left|S_{i j}\right|=10^{\frac{S_{32}[\mathrm{~dB}]}{20}}
$$

The logarithmic magnitude is typically used to respond to skewness towards large values, and to show percent change or multiplicative factors. Therefore, given that the linear magnitude representation is more adequate than the logarithmic form to represent accurate values, this will be the representation form exploited in this thesis. 
Given the transversal symmetry of the cell, we observe the following symmetries in the $S$ parameters:

$$
\begin{aligned}
\left|S_{11}\right| & =\left|S_{22}\right|=\left|S_{33}\right|=\left|S_{44}\right| \\
\left|S_{12}\right|=\left|S_{14}\right|=\left|S_{21}\right| & =\left|S_{41}\right|=\left|S_{23}\right|=\left|S_{43}\right|=\left|S_{32}\right|=\left|S_{34}\right| \\
\left|S_{13}\right| & =\left|S_{31}\right|=\left|S_{24}\right|=\left|S_{42}\right|
\end{aligned}
$$

In Figure 2.19, the $S$ parameters at p1 of the perturbed -solid lines- and unperturbed -dash lines- middle cell due to the presence of the conductor wire at the geometric centre are plotted. As expected from the Slater's perturbation theorem, a frequency shift of around $0.4 \mathrm{GHz}$ is observed due to the presence of the wire. In Figure 2.20, the same measurements are plotted for the whole TD24 AS, where the dash lines represent the unperturbed case and the solid lines represent the perturbed AS. The reflections from the other cells are marked with black circles -this result will be justified in the next section- while the modes coupling to the wire are marked as purple circles.

In order to save computational time, the smallest significant simulation model should be used. For this purpose, the $\mathrm{S}$ parameter simulation strategy adopted to estimate the effect of the other cells surrounding the middle cell (cell number 13) is sketched in Figure 2.21. The $S$ parameters are simulated for cells number 12, 13 and 14. Later, five cells are considered including cells 11,12,13,14 and 15. And finally, the $S$ parameters are obtained for the whole structure consisting on 26 cells where the two coupling input and output cells are also considered. The objective is to look for a frequency range where the $S$ parameters are not ringing (black and green circles in Figure 2.20 and black circles in Figure 2.22). This frequency range will be used to perform the measurement of the EM centre. The simulation results are shown in Figure 2.22. The obtained plots differ from each other on the number of reflections that is equal to the number of simulated cells. Such reflections are grouped around frequencies $17.2 \mathrm{GHz}$ and $18.2 \mathrm{GHz}$, marked in the plots as black circles, so we can conclude that these are two frequency zones to avoid in the measurement of the $S$ parameters.

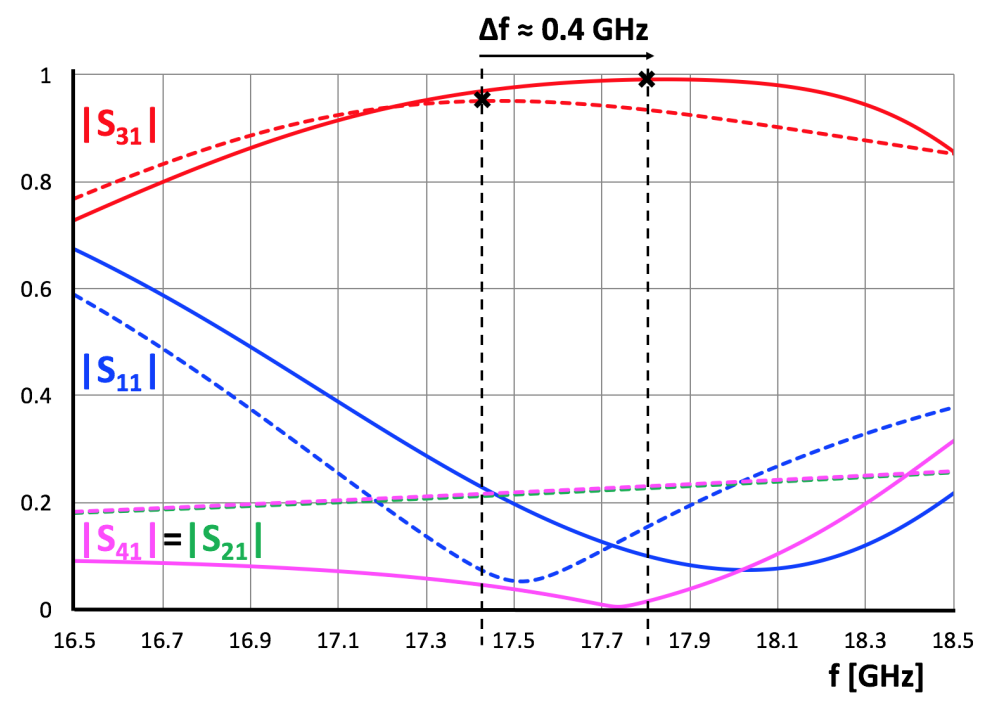

Figure 2.19: $S$ parameters showing the frequency shift of the unperturbed (dash lines) and perturbed (solid lines) middle cell. 


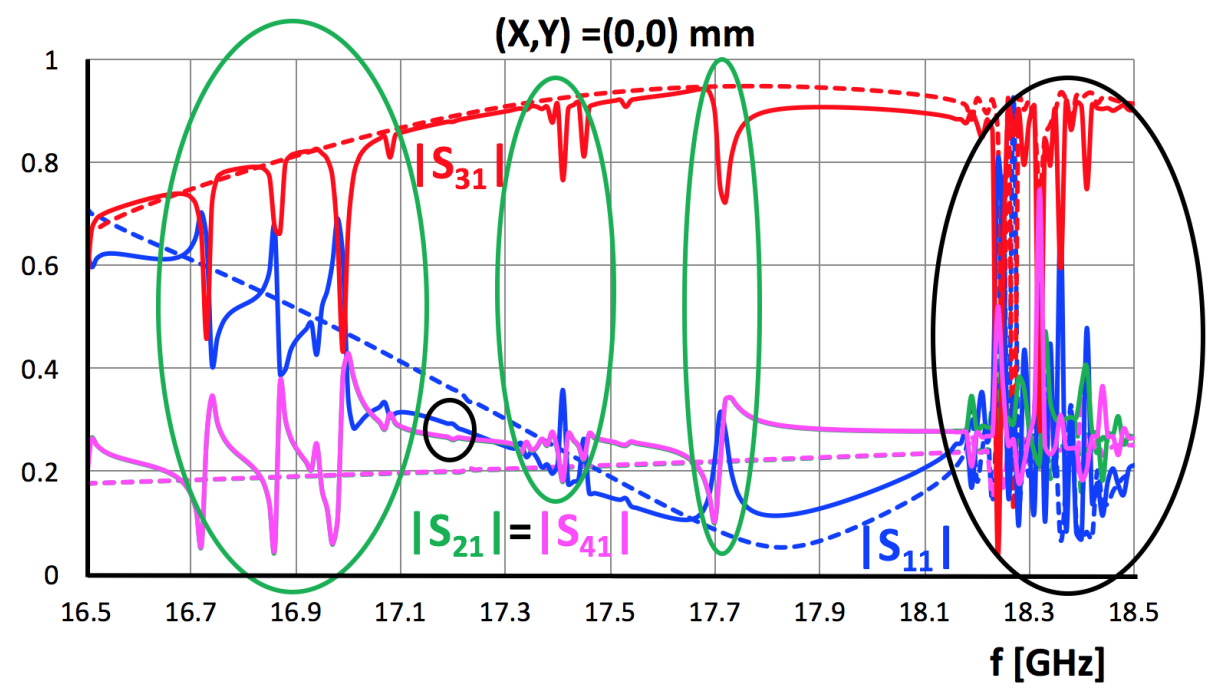

Figure 2.20: $S$ parameters in the middle cell when simulating the whole TD24. The dash lines correspond to the unperturbed AS while the solid lines correspond to the perturbed AS with a wire. Green circles represent the modes coupling to the wire and black circles, the reflection from the cells. The legend $(\mathrm{X}, \mathrm{Y})$ indicates the wire position coordinates.

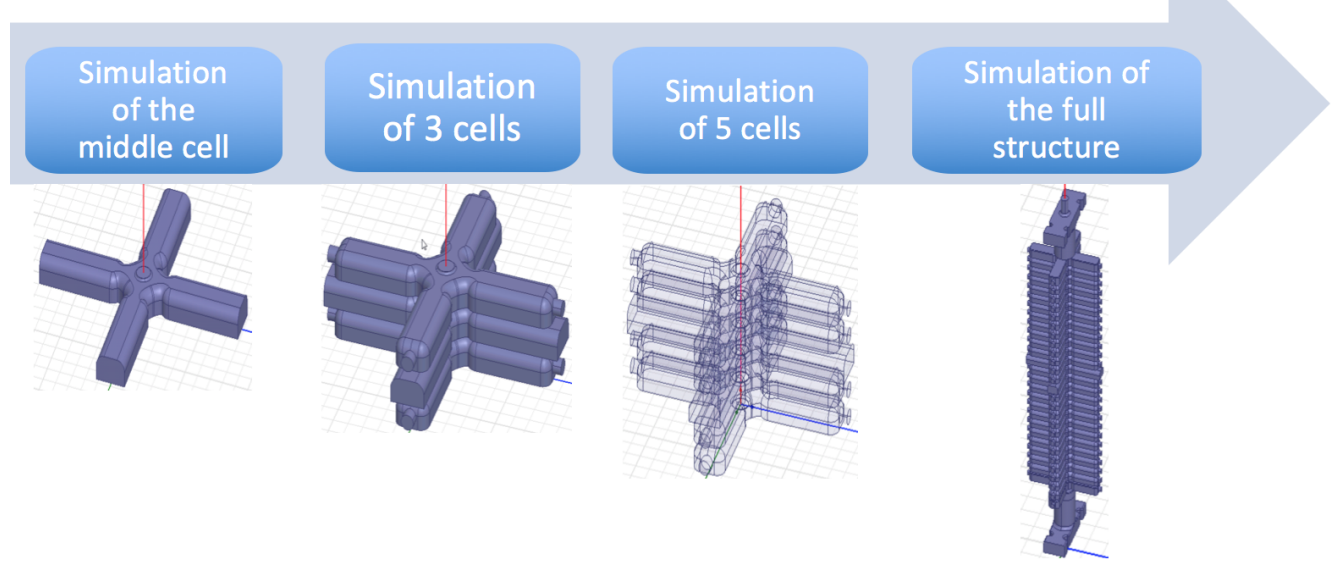

Figure 2.21: Simulation studies performed using different models of the CLIC TD24 AS, going from one single cell, to three, to five and ending up with the whole structure. 


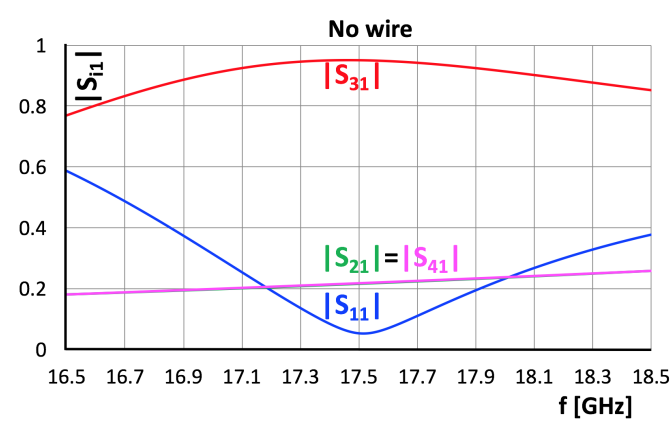

(a)

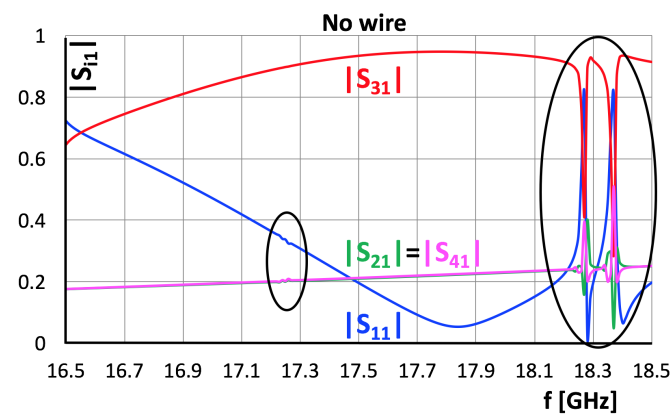

(c)

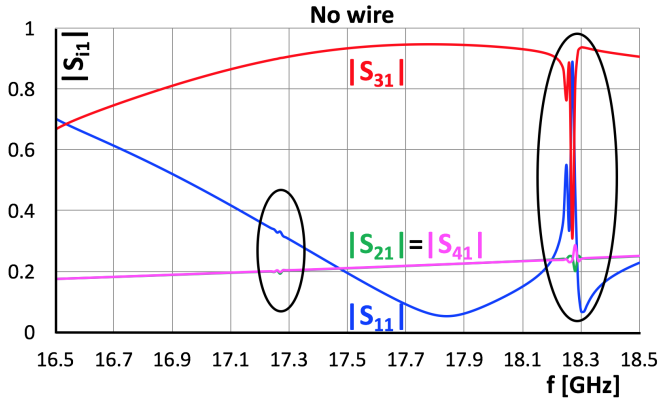

(b)

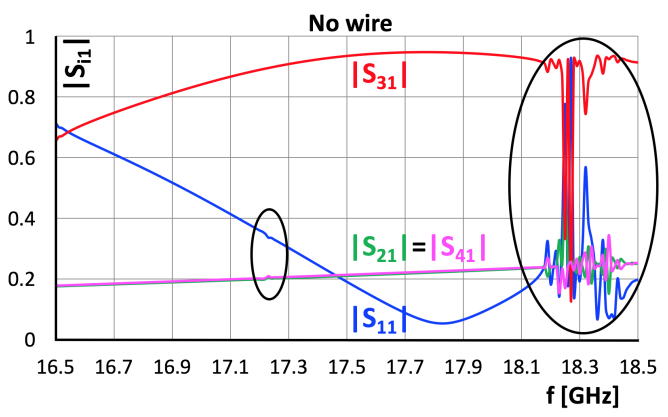

(d)

Figure 2.22: Simulated $S$ parameters without wire at cell 13 of a model consisting on: (a) cell 13; (b) cells 12, 13, 14; (c) cells 11, 12, 13, 14, 15; (d) all cells of the TD24. Black circles represent the reflection from the cells surrounding cell 13 . The legend indicates the absence of wire.

From this study, the different frequency ranges of interest to perform the measurement of the $S$ parameters are the following: $(17-17.2] \mathrm{GHz},[17.2-17.3) \mathrm{GHz},(17.5-$ 17.7] GHz, [17.8-18.2] GHz, where an open interval is indicated as parentheses and a closed interval is denoted as square brackets. In order to verify this, and for the completeness of this study, we simulate the whole TD24 and see how the wire behaves at these frequency ranges. The copper wire is inserted in the simulation model of the whole structure and is moved in $X$ in a range of $4 \mathrm{~mm}$ with steps of $500 \mu \mathrm{m}$. This way, the wire is used as a passive object, draining power from the cell according to its position. The value of the $S$ parameters change at each position when exciting the AS from port 1 change at each wire position as plotted in Figure 2.23. As we could expect from the symmetry of the middle cell, $\left|S_{41}\right|$ and $\left|S_{21}\right|$ have the same value when the wire is placed at the geometric centre. In other words, when the wire is in the geometric centre of the AS, the same amount of power is driven to the adjacent ports -the perturbation created by the wire is minimised and is very similar to the case without a wire. Also, at any given frequency $f$, the dependence of $\left|S_{41}\right|$ with respect to the wire position $Y$ is opposite to $\left|S_{21}\right|$. This result can be written as:

$$
\left|S_{41}\right|(f, Y)=\left|S_{21}\right|(f,-Y)
$$

This means that it is worth investigating deeper the relationship $S_{41}-S_{21}$ in order to develop a conceptual design algorithm to localise the EM centre. Concerning the reflexion $\left|S_{11}\right|$ and the transmission of the opposite port $\left|S_{13}\right|$, the variations when moving the wire 

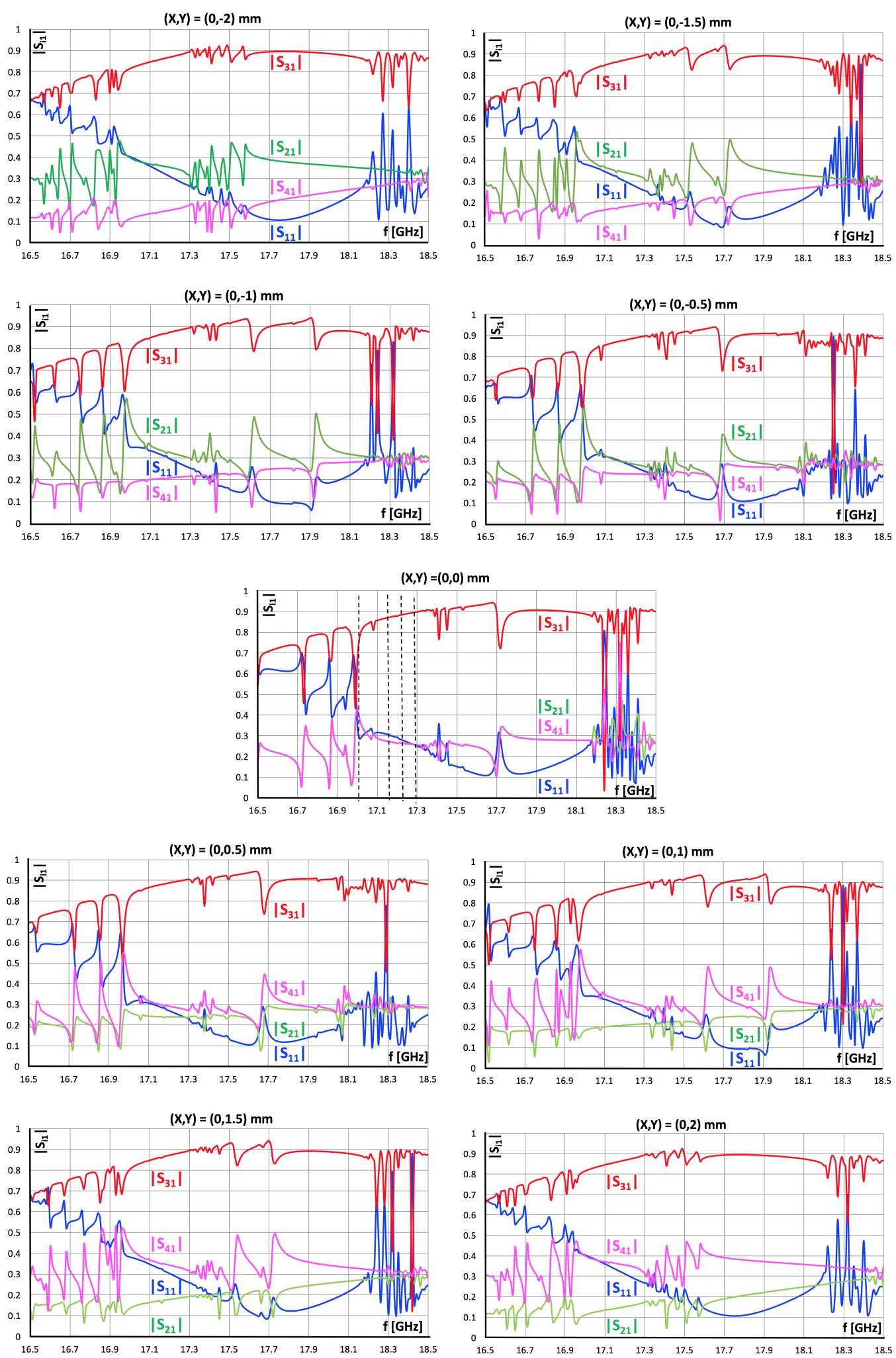

Figure 2.23: Simulation of $\left|S_{11}\right|,\left|S_{21}\right|,\left|S_{31}\right|$ and $\left|S_{41}\right|$ with respect to the frequency when a wire moves along $Y$ inside the TD24 AS. The legend represents the wire position coordinates. 


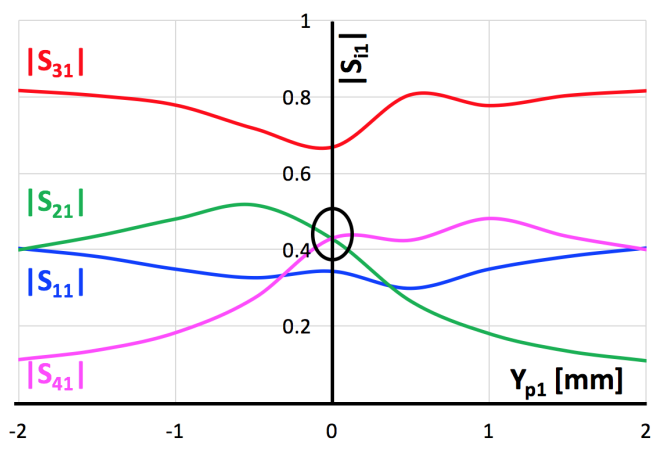

(a)

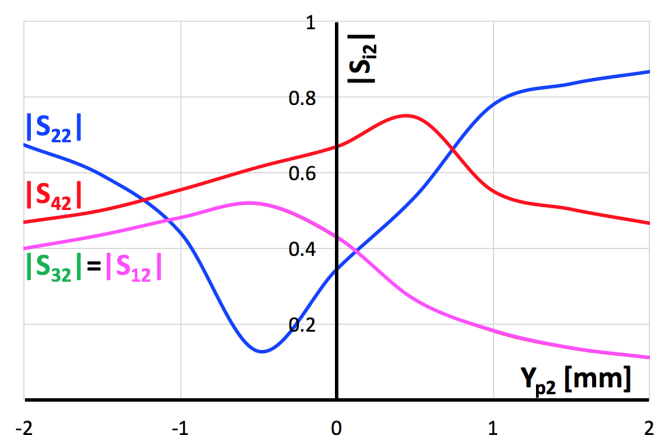

(b)

Figure 2.24: (a) Simulated $S$ parameters for the whole structure with respect to the wire position in $Y$ at $17 \mathrm{GHz}$ when exciting from port 1 and (b) when exciting from port 2.

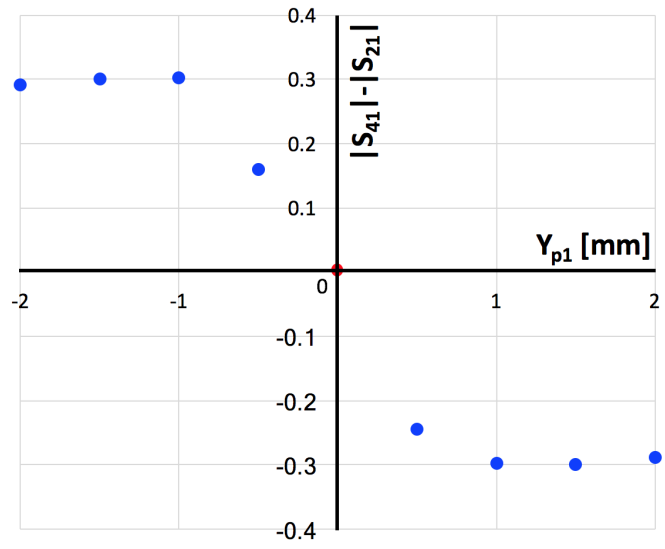

Figure 2.25: Simulated $\left|S_{41}\right|-\left|S_{21}\right|$ of the whole structure with respect to the wire position in $Y$ at $17 \mathrm{GHz}$.

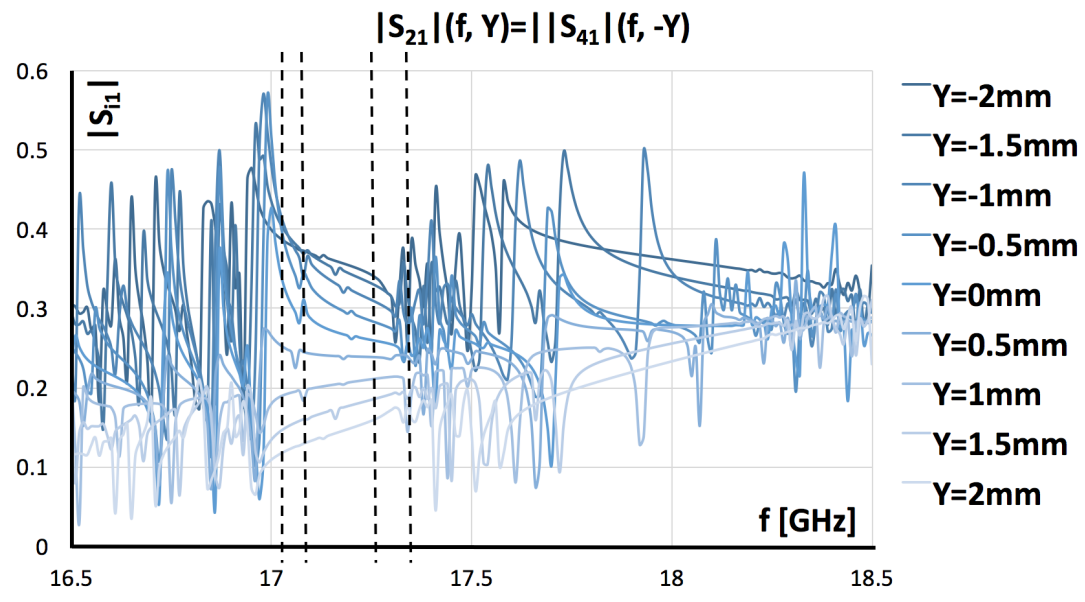

Figure 2.26: Simulated $\left|S_{21}\right|$ and $\left|S_{41}\right|$ of the whole structure with respect to the frequency at different wire positions in $Y$. The legend represents the wire position in the vertical coordinate. 
are smaller and behave different than in the previous case. This result was already observed as an asymmetry of the EM field simulations in Figure 2.18. For this reason, we decided to exploit the symmetry between adjacent ports. The dependence of $Y$ with input p1 - $\left|S_{11}\right|,\left|S_{21}\right|,\left|S_{31}\right|$ and $\left|S_{41}\right|-$ at $17 \mathrm{GHz}$ can be seen in Figure 2.24 (a). If we take $\left|S_{41}\right|-\left|S_{21}\right|$, that we define as Asymmetry Measurement from p1, $\left(A M_{1}\right)$, as plotted in Figure 2.25 we observe that this quantity is zero at the position of the geometric centre, marked as a black circle. The same result is obtained for input port 3 (p3) when performing an Asymmetry Measurement from p3, $\left(A M_{3}\right),\left|S_{43}\right|-\left|S_{23}\right|$. Resolving the same symmetric problem, the logic calculations to be performed to obtain the EM centre in the $X$ axis are: $\left|S_{32}\right|-\left|S_{12}\right|$, or Asymmetry Measurement from port 2 (p2), $\left(A M_{2}\right)$, and $\left|S_{34}\right|-\left|S_{14}\right|$, Asymmetry Measurement from port 4 (p4), (AM $)$.

As observed in Figure 2.23 the variation of the $\left|S_{21}\right|$ and $\left|S_{41}\right|$ parameters is not the same at all frequencies. This can be seen more clearly in Figure 2.26, where all simulated $\left|S_{21}\right|(f, Y)$ and $\left|S_{41}\right|(f,-Y)$ are plotted with respect to the frequency. From this plot, we observe that $\left|S_{21}\right|$ and $\left|S_{41}\right|$ are linear and monotonic in the frequency ranges: (17-17.2] GHz and [17.2-17.3) GHz, marked as dash lines in Figure 2.26. Since sensitivity decreases with frequency, we chose the smallest frequency from the indicated ranges: $17 \mathrm{GHz}$.

In summary, we will obtain the EM centre of the middle cell in both axes $(X$ and $Y)$ at the positions derived from $\mathrm{Eq}(2.57)$ as:

$$
\begin{aligned}
& \left|S_{21}\right|(Y)=\left|S_{41}\right|(-Y) \text { at } f=17 \mathrm{GHz} \\
& \left|S_{23}\right|(Y)=\left|S_{43}\right|(-Y) \text { at } f=17 \mathrm{GHz} \\
& \left|S_{32}\right|(X)=\left|S_{12}\right|(-X) \text { at } f=17 \mathrm{GHz} \\
& \left|S_{34}\right|(X)=\left|S_{14}\right|(-X) \text { at } f=17 \mathrm{GHz}
\end{aligned}
$$

As we have seen in Figure 2.22, the $S$ parameters obtained for one single cell without wire were very similar as for 24 cells, except for the two ringing zones. The golden frequency is also satisfied in the case of one cell as seen in Figure 2.27, where $\left|S_{41}\right|-\left|S_{21}\right|$ is plotted with respect to the position of a moving wire in $Y$ at different frequencies. For doing this simulation in the middle cell, the wire position changed from $(X, Y)=(0,-500) \mu \mathrm{m}$ to $(X, Y)=(0,500) \mu \mathrm{m}$ in steps of $50 \mu \mathrm{m}$. It is observed, that at $17.1 \mathrm{GHz}$ the sensitivity is smaller than at $16.9 \mathrm{GHz}$. However, $16.9 \mathrm{GHz}$ does not belong the frequency range without ringing for the whole structure, as seen in Figure 2.26. So, we could conclude that we chose $17 \mathrm{GHz}$ as a good compromise. The next simulations in HFSS were performed only in the middle cell of the TD24 in order to save simulation resources and time.

We have identified the $S$ parameters carrying out a linear relationship with the wire displacement around the EM centre of the TD24. In the next simulation, we zoomed in around the EM axis and simulated a wire moving in the vertical axis with steps of $1 \mu \mathrm{m}$ in order to study the linearity of the $S$ parameters giving the position of the EM axis and in order to develop a measurement algorithm. With this simulation, we could also find the maximum resolution of the simulation measurements, and determine the specifications of an experimental test bench, able to localise the EM axis within the accepted accuracy. 


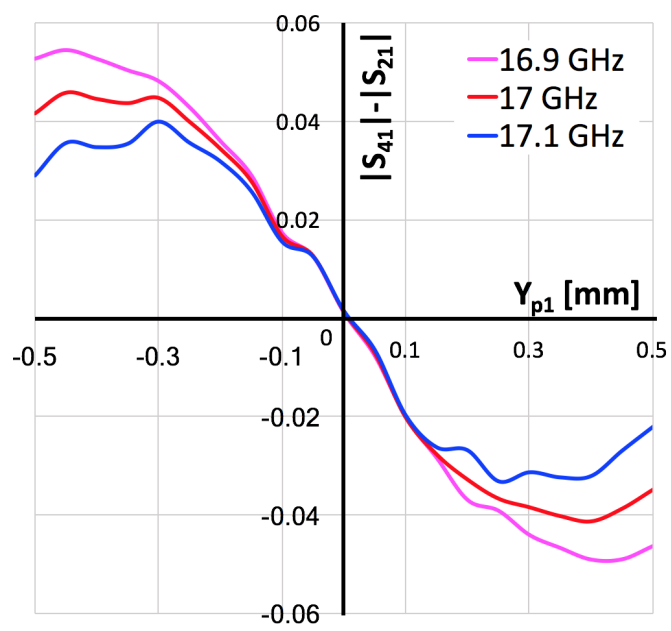

Figure 2.27: Simulated $\left|S_{41}\right|-\left|S_{21}\right|$ at different frequencies in the middle cell.

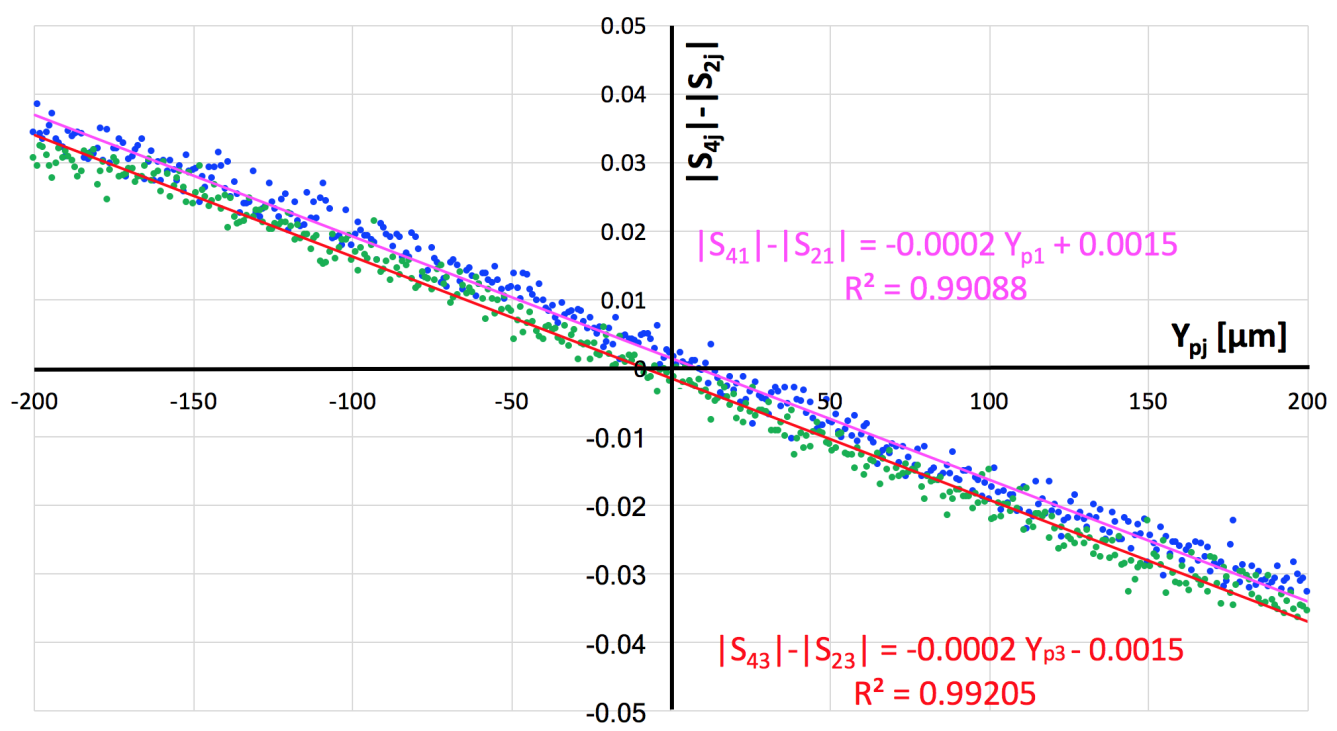

Figure 2.28: $\left|S_{4 j}\right|-\left|S_{2 j}\right|$ of a wire moving in $Y$ in the middle cell and linear fit.

The $S$ parameters of interest at $\mathrm{p} 1$ and $\mathrm{p} 3$ in Figure 2.28 are represented as blue dots and green dots, respectively. Around the centre, the dependence is linear, so we perform a linear regression of the simulated measurements around this zone, and the two equations in pink and red are obtained. As we could see from the goodness of the fit $\left(R^{2}\right)$, the linear regression is well adapted, so the errors in the simulated measurements are caused by numerical noise. Being $m$ the slope of the line and $a$ the intercept point in the vertical axis, we can rewrite the linear fit of $\left|S_{41}\right|-\left|S_{21}\right|$ (pink equation in Figure 2.28) as:

$$
\left|S_{41}\right|-\left|S_{21}\right|=m_{y, p 1} \cdot Y_{p 1}+a_{y, p 1}
$$

When Eq 2.58 is fulfilled, the position of the EM centre in the $Y$ axis will be obtained at 
$Y_{p 1}$. In a similar way for $\mathrm{p} 3$, we rewrite the equation, red line in Figure 2.28, as:

$$
\left|S_{43}\right|-\left|S_{23}\right|=m_{y, p 3} \cdot Y_{p 3}+a_{y, p 3}
$$

The position of the EM centre is obtained at $Y_{p 3}$ following Eq (2.59).

The slopes of both linear regressions are the same, $m_{y, p 1}=m_{y, p 3}$, and the intercept point in the vertical axis, $a_{y, p 1}$ and $a_{y, p 3}$, have the same values but differ in the sign. This means that the EM centre is found at different positions, $Y_{p 1}$ and $Y_{p 3}$, symmetric from zero. This result is probably coming either from the wire radius or from the excitation asymmetry as seen in Figure 2.18, so we calculate the position of the EM centre in $Y$ as: $Y_{p 13}=\left(Y_{p 1}+Y_{p 3}\right) / 2$ to make it coincide with the position of the geometric centre in simulations. The difference on the positioning as measured by each port could be accounted as a theoretical accuracy limit, with a value of $\pm 7.5 \mu \mathrm{m}$.

Moving the wire along the horizontal axis, we can rewrite in a similar way the linear regression of the simulated $S$ parameters from ports 2 and 4 as:

$$
\begin{aligned}
& \left|S_{32}\right|-\left|S_{12}\right|=m_{x, p 2} \cdot X_{p 2}+a_{x, p 2} \\
& \left|S_{34}\right|-\left|S_{14}\right|=m_{x, p 4} \cdot X_{p 4}+a_{x, p 4}
\end{aligned}
$$

Their corresponding solutions of the EM centre in $X$ are given by $X_{p 2}$ and $X_{p 4}$, when Eq 2.60 and 2.61 are fulfilled, respectively. The same results as before are reproduced, so we calculate the position of the EM centre in $X$ as: $X_{p 24}=\left(X_{p 2}+X_{p 4}\right) / 2$. The position of the EM axis is, therefore, given by: $(\mathbf{X}, \mathbf{Y})=\left(X_{p 24}, Y_{p 13}\right)$.

From this study, the conceptual design algorithm to obtain the position of the EM axis will be performed following the steps:

1. Move the AS along $X$.

2. Measure $S_{32}, S_{12}, S_{34}, S_{14}$.

3. Calculate $\left|S_{32}\right|-\left|S_{12}\right|(\mathrm{Eq}(2.64))$ and $\left|S_{34}\right|-\left|S_{14}\right|(\mathrm{Eq}(2.65))$.

4. Calculate $X_{p 2}$ and $X_{p 4}$.

5. Obtain $X_{p 24}=\left(X_{p 2}+X_{p 4}\right) / 2$.

6. Bring the AS to $\mathrm{X}=X_{p 24}$ and move along $Y$.

7. Measure $S_{41}, S_{21}, S_{43}, S_{23}$.

8. Calculate $\left|S_{41}\right|-\left|S_{21}\right|(\mathrm{Eq}(2.62))$ and $\left|S_{43}\right|-\left|S_{23}\right|(\mathrm{Eq}(2.63))$.

9. Calculate $y_{p 1}$ and $y_{p 3}$.

10. Obtain $Y_{p 13}=\left(Y_{p 1}+Y_{p 3}\right) / 2$.

11. Iterate until both $X_{p 24}$ and $Y_{p 13}$ converge.

12. The position of the EM axis is $(\mathrm{X}, \mathrm{Y})=\left(X_{p 24}, Y_{p 13}\right)$. 


\subsubsection{Accuracy and resolution study}

In general, a minimum of 6 simulated points are enough to obtain a good regression line [81]. However, we strive for a very accurate measurement of the EM, so we studied the resolution limit with HFSS. For this purpose, we performed a linear fit of the simulation results from Figure 2.28 using different step sizes, or resolutions, and a wire travel range in $Y$ of $400 \mu \mathrm{m}$. For each resolution, ranging from 1 to $100 \mu \mathrm{m}$, the EM centre $Y_{p 13}$ in the vertical axis was calculated. In all of the cases considered for this study, it was observed that the slopes of the regression lines did not change. However, we found $Y_{p 13}=4 \mu \mathrm{m}$ for a step size of $100 \mu \mathrm{m}$, so the EM centre was deviated around $4 \mu \mathrm{m}$ with respect to the geometric centre. The results obtained for $50 \mu \mathrm{m}$ steps were still within the accuracy limit, so we could obtain a reliable measurement of the EM centre in a vertical range of $400 \mu \mathrm{m}$ with 9 points. In a similar way, with a smaller travel range of $100 \mu \mathrm{m}$, we obtained a smaller deviation $Y_{p 13}=0.75 \mu \mathrm{m}$ with a resolution of $20 \mu \mathrm{m}$. We conclude, that we can obtain an accurate measurement with an error below $1 \mu \mathrm{m}$ when moving the wire along $100 \mu \mathrm{m}$ around the geometric centre with a minimum step size of $20 \mu \mathrm{m}$.

These results are an indication of the experimental set-up specifications necessary to perform the measurement of the EM centre. In particular, the elements allowing the movement of the AS, or linear stages, around the fixed wire needs a minimum incremental step of $20 \mu \mathrm{m}$ since we strive for a very accurate measurement of the centre around the micron. Such elements are easily available in the market. Indeed, we will see in Chapter 4, that our experimental set-up will be composed by two linear stages with a minimum incremental step of $0.1 \mu \mathrm{m}$. This is more than enough to validate the method and achieve the required measurement accuracy.

\subsubsection{Characterisation of errors}

HFSS is also used as a tool to characterise the sources of errors in the measurement of the EM axis in the TD24. Several hardware imperfections were simulated giving more realistic $S$ parameters results in view of the laboratory measurements.

As a first study, we will simulate the effect of realistic set-up in which the wire moves in a line that is not orthogonal to the geometric axes of the middle cell. After that, we will simulate a wire that is tilted with respect to the longitudinal axis $Z$. And finally, we will present the design of the required tapered transitions, and we will study how their coupling with the middle cell through the damping waveguides affects the $S$ parameters measurements.

\subsubsection{Wire offset with respect to the transverse plane}

We considered first a wire with different offsets moving orthogonal to the geometric axes in the middle cell, as shown in Figure 2.29 (a). Several simulations were performed when moving the wire along $Y$ in a travel range of $400 \mu \mathrm{m}$ while $X$ was fixed to a few positions different from $X=0$. The resulting $\left|S_{4 j}\right|-\left|S_{2 j}\right|, j=1,3$, are plotted in Figure 2.30 and a couple of results could be observed. The slopes increase with the wire offset. Indeed, 
when the wire moves along $Y$ and remains fix at $\mathrm{X}=0 \mu \mathrm{m}$, both $\left|S_{4 j}\right|-\left|S_{2 j}\right|, j=1,3$, have the same slopes. However, when the wire is off-centred in $X$, different slopes are obtained. For instance, at $X=0 \mu \mathrm{m}$ the sensitivity is $\frac{0.1}{600 \mu \mathrm{m}}$, while it is $\frac{0.3}{600 \mu \mathrm{m}}$ at $\mathrm{X}=500 \mu \mathrm{m}$. Note also that the combination of the $S$ parameters are around zero at the position of the geometric centre, even for off-centred wires. Therefore, we can estimate how close the wire is from the centre by comparing the slope of both measurements from opposite ports.

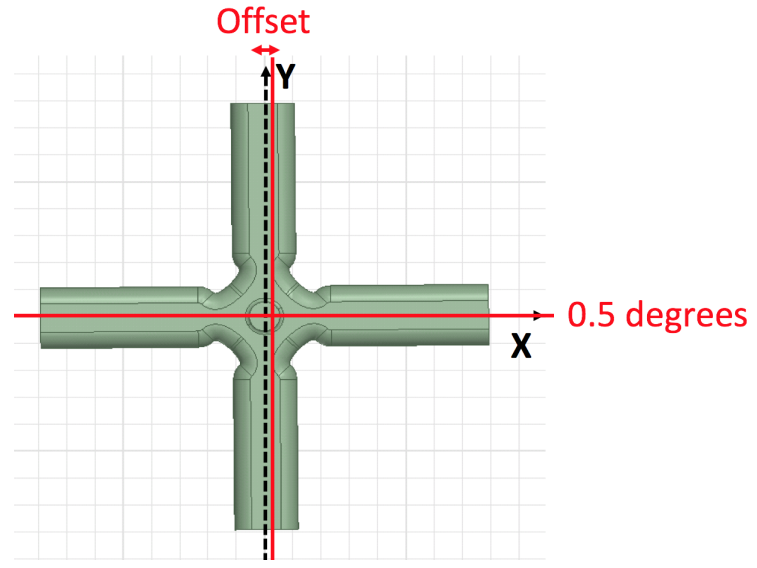

(a)

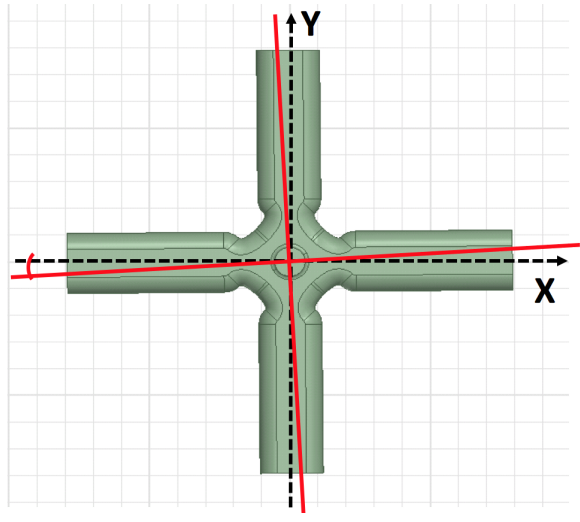

(b)

Figure 2.29: (a) Top view of the middle cell in HFSS with an offset in the $X$ axis and (b) rotated 0.5 degrees around the $Z$ axis. The red lines represent the wire travel range, that is deviated from the geometric axes (dashed lines).

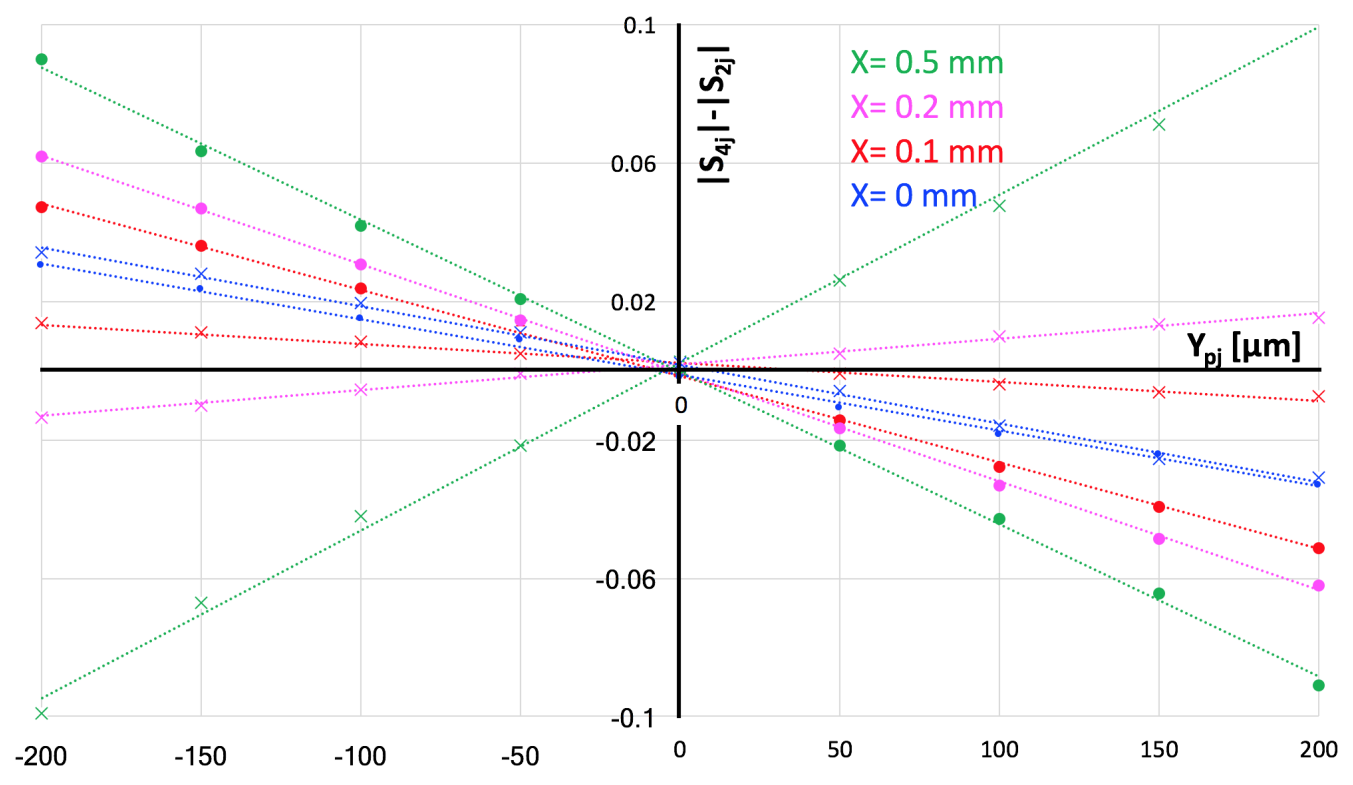

Figure 2.30: $\left|S_{41}\right|-\left|S_{21}\right|$ (crosses) and $\left|S_{43}\right|-\left|S_{23}\right|$ (circles) in the middle cell for different wire offsets in $X$ while moves along $Y$. The legend indicates the wire position in the horizontal coordinate. 
A more realistic configuration, shown in Figure 2.29 (b), was simulated in which the wire moves in two orthogonal lines (red lines) that are rotated 0.5 degrees in $Z$ with respect to the geometric axes of the middle cell (black dash lines). For this simulation study, the algorithm was iterated several times and the EM centre was calculated. In the first iteration, the wire moved along the new travel range or red lines, from $(\mathrm{X}, \mathrm{Y})=(0$,200) $\mu \mathrm{m}$ to $(X, Y)=(0,200) \mu \mathrm{m}$ in steps of $20 \mu \mathrm{m}$. The position of the centre was found at $Y_{p 13}=1.25 \mu \mathrm{m}$. After that, the wire was moved from $(X, Y)=(-200,1) \mu \mathrm{m}$ to $(X, Y)=(200,1) \mu \mathrm{m}$ in steps of $20 \mu \mathrm{m}$. We obtained $X_{p 24}=2 \mu \mathrm{m}$. A second iteration was performed in which the wire was moved again along $400 \mu \mathrm{m}$ with the same step size. The result of $Y_{p 13}$ was zero. Finally, the wire was moved along $X$ with the new position of $Y_{p 13}$ and we obtained $X_{p 24}=0.5 \mu \mathrm{m}$. This position was given for the next iteration, and we obtained again $Y_{p 13}=0$. This means, that in a realistic set-up, we could approach to the position of the EM centre by iterating the algorithm probably more than once.

\subsubsection{Tilted wire in the longitudinal axis}

The configuration of a tilted wire in the longitudinal axis of the middle cell is shown in Figure 2.31. The tilt is settled in the $X$ axis and the tilt angle is represented by $\theta$. The wire was moved from $(X, Y)=(0,-200) \mu \mathrm{m}$ to $(X, Y)=(0,200) \mu \mathrm{m}$ in steps of $50 \mu \mathrm{m}$. The resulting $\left|S_{4 j}\right|-\left|S_{2 j}\right|, j=1,3$, with respect to the tilt are represented in Figure 2.32. We observe that $A M_{1}$ and $A M_{3}$ have different slopes, very similarly as the effect of an offset. These results should be reproduced at $\mathrm{p} 2$ and $\mathrm{p} 4$ when the tilt is established in the vertical axis. So we conclude that the localisation of the EM axes is not inherent to the tilt and only sensitivity at ports localised in the direction of the tilted axis is affected. For all the simulated $\theta, A M$ are around zero at the position of the geometric centre. In reality, a tilt is expected at both planes at the same time in the test set-up and need to be controlled. The procedure and tools for its minimisation are explained in Chapter 4.

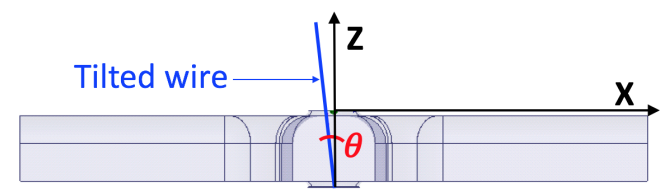

Figure 2.31: Side view of the middle cell in HFSS with tilted wire in $X$.

\subsubsection{Coupling between the middle cell and the tapered transitions}

To preserve the symmetry in the middle cell, transition waveguides -also called tapered transitions- to couple the damping waveguides were developed as part of this work. A tapered transition has been designed using HFSS, and has been registered at CERN EDMS under CDD number CLIASTND0136 [92]. Its dimensions are shown in Figure 2.33.

The design process using $\mathbf{S}$ parameter simulations in HFSS was done as follows. Both end sections were drawn in HFSS and were connected through a variable waveguide length with a linear transformation. This design was chosen from the three transformations explained in [82] for manufacturing simplicity. Different waveguide lengths lead to 


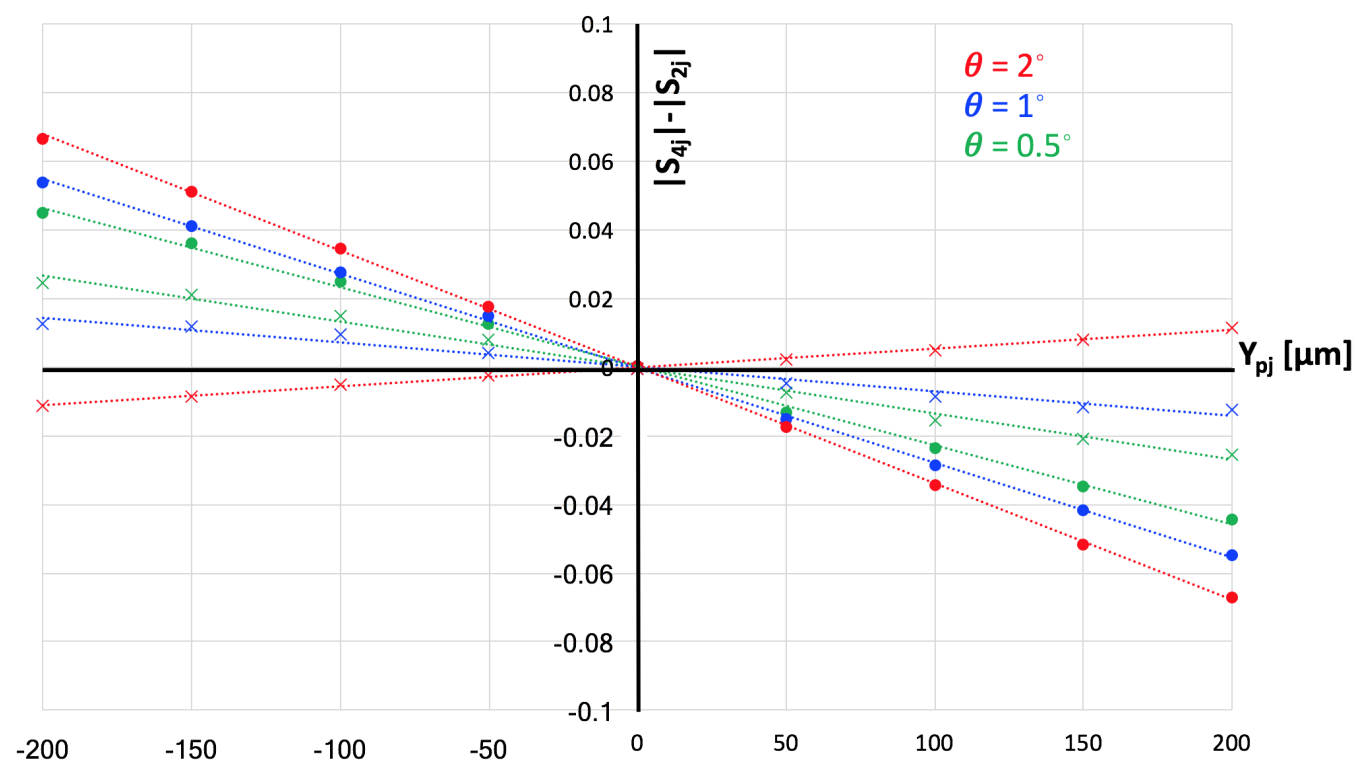

Figure 2.32: $\left|S_{41}\right|-\left|S_{21}\right|$ (crosses) and $\left|S_{43}\right|-\left|S_{23}\right|$ (circles) in the middle cell for different tilt angles $\theta$ in the $X$ axis.

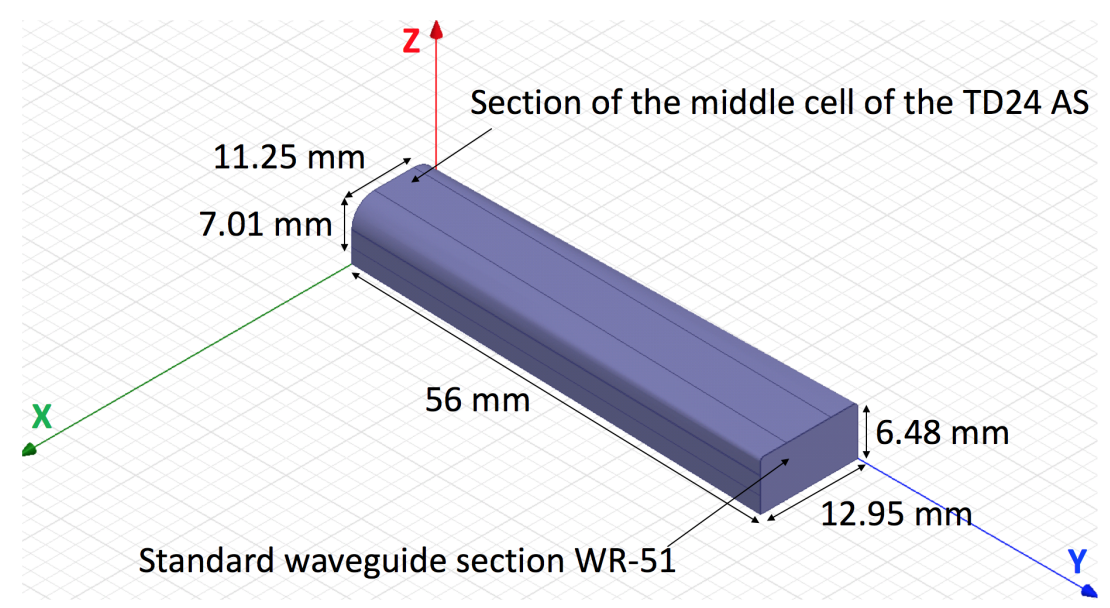

Figure 2.33: Model of the tapered transition designed with HFSS and dimensions of the cross sections of the middle cell and the standard waveguide WR-51.

different transmitted and reflected signals when exciting from the port with cross section WR-51. In general, when designing low-reflection microwave components, an accepted value for minimum reflection coefficient, $S_{11}$, is about $-40 \mathrm{~dB}$. So, the goal was to optimise the tapered length with respect to the reflection limit in the frequency range of interest. Figure 2.34 illustrates the simulated S parameter obtained for the optimised length of $56 \mathrm{~mm}$ when port 1 is defined at section WR-51 and port 2 refers to the middle cell section. Note that $S_{11}$ is below $-40 \mathrm{~dB}$ in the range of frequencies of interest and that the taper is reciprocal, as we find that $S_{12}=S_{21}$ and $\left|S_{11}\right|=\left|S_{22}\right|$. 


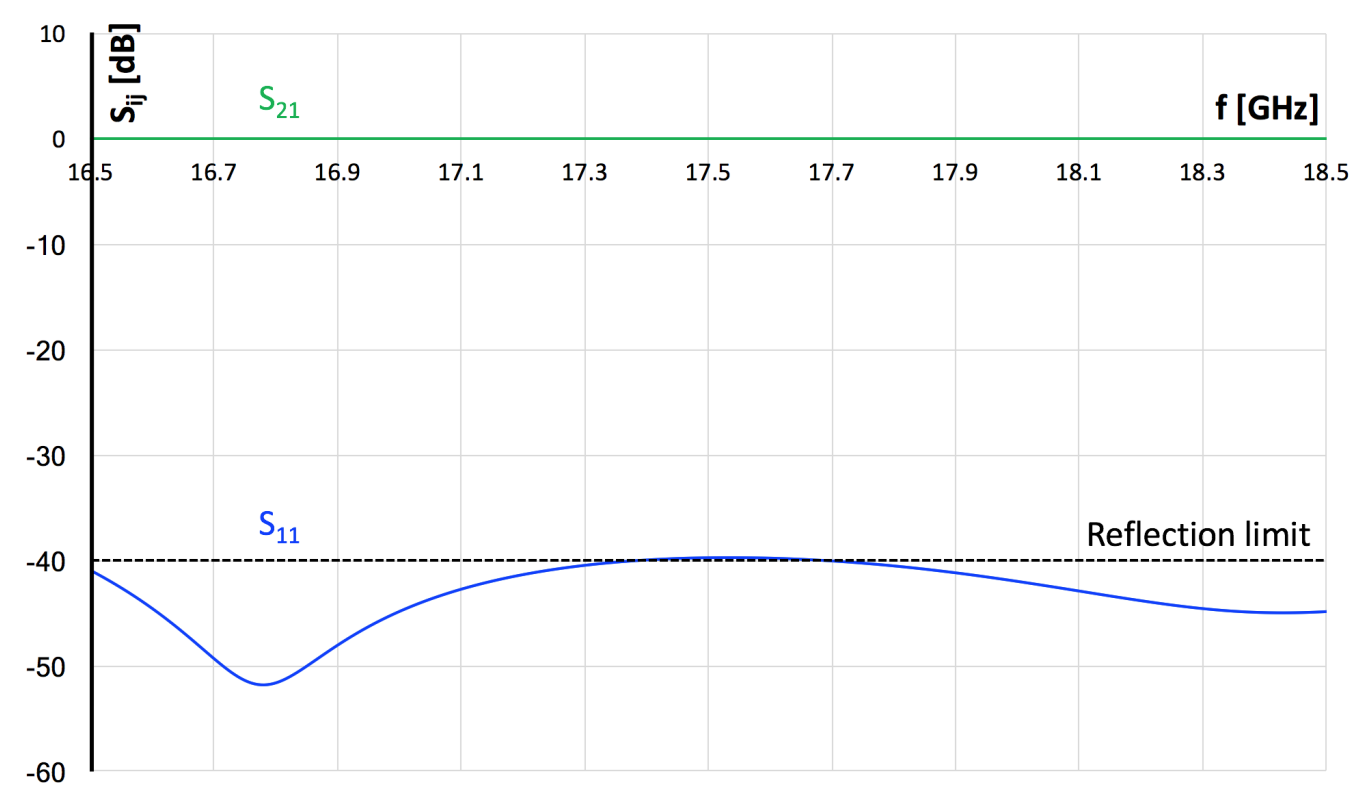

Figure 2.34: Simulated $S$ parameters of the tapered transition with HFSS.

To study in a simplistic way how the coupling could affect the measurement of the EM axis, we connected a tapered transition in HFSS to one piece of damping waveguide as shown in Figure 2.35. P1 was defined at the end of the tapered transition while p2 was defined at the end of the damping waveguide. The simulated transmission $\left|S_{21}\right|$ and reflection $\left|S_{11}\right|$ are plotted with respect to the shift in $X, \Delta \mathrm{X}$, and $Z, \Delta \mathrm{Z}$, in Figures 2.36 and 2.37, respectively. We observe that, as expected, the better the coupling, the lower reflections and the better transmission between ports. The results in Figure 2.36 are symmetric for negative positions of the tapered in $X$, being the most affecting axis to the $S$ parameters.

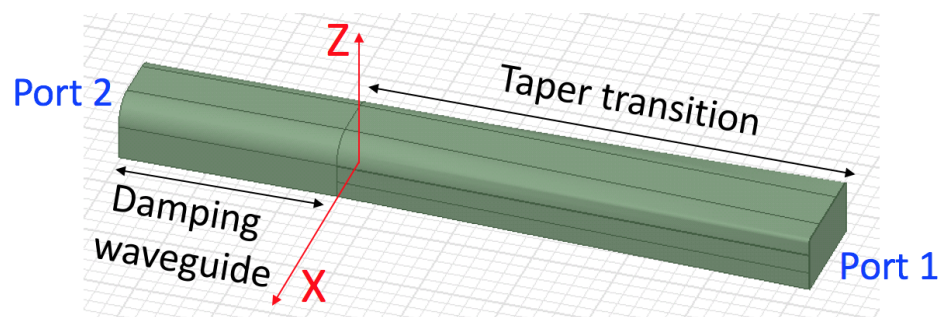

Figure 2.35: Tapered transition coupled to a damping waveguide section. 


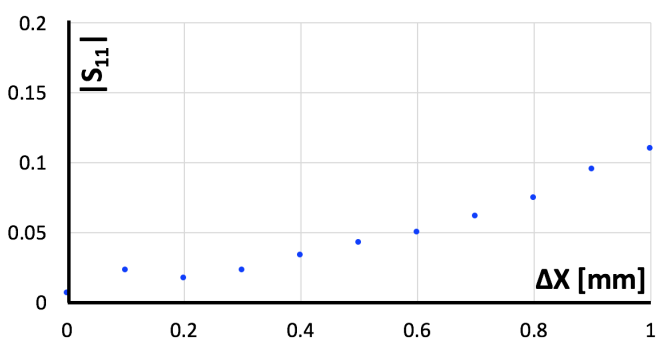

(a)

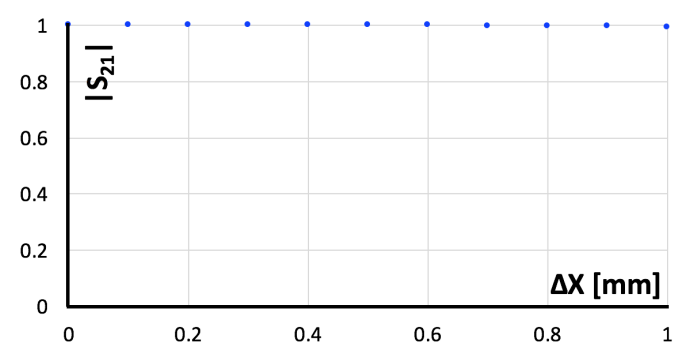

(b)

Figure 2.36: (a) $\left|S_{11}\right|$ at $17 \mathrm{GHz}$ with respect to the displacement in $X$ of the tapered transition coupled to a piece of damping waveguide and (b) similar for $\left|S_{21}\right|$.

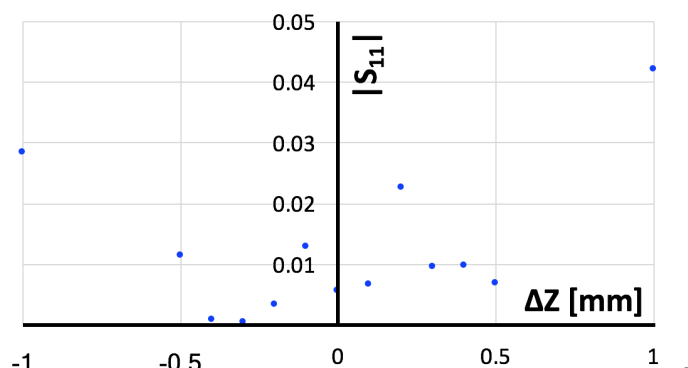

(a)

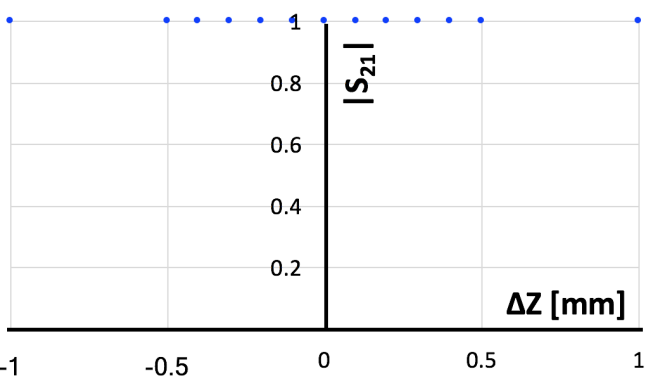

(b)

Figure 2.37: (a) $\left|S_{11}\right|$ at $17 \mathrm{GHz}$ with respect to the displacement in $Z$ of the tapered transition coupled to a fragment of damping waveguide and (b) similar for $\left|S_{21}\right|$.

The global effect of the coupling is simulated using four tapered transitions (tapers 1 to 4 in Figure 2.38) connected at the end of the damping waveguides in the middle cell. The new port configuration is such that $\mathrm{p} 1$ is defined at the end of taper $1, \mathrm{p} 2$ is defined at the end of taper 2, and so on. For this study, a perfect coupling between the middle cell and all tapered transitions are considered. A wire is moved from $(X, Y)=(0,-200) \mu \mathrm{m}$ to $(\mathrm{X}, \mathrm{Y})=(0,200) \mu \mathrm{m}$ in steps of $50 \mu \mathrm{m}$. The $S$ parameters obtained are plotted in Figure 2.39 where the blue crosses and the blue circles represent the measured $\left|S_{41}\right|-\left|S_{21}\right|$ and $\left|S_{43}\right|-\left|S_{23}\right|$ at ports 1 and 3, respectively. We find that the linear regression of $\left|S_{4 j}\right|-$ $\left|S_{2 j}\right|, j=1,3$, cross the horizontal axis at symmetric positions of the geometric centre, so $Y_{p 13}=0$.

After that, the simulation was repeated with the position of taper 3 shifted $+1 \mathrm{~mm}$ in the $Z$ axis. The resulting $S$ parameters at ports 1 and 3 are shown in Figure 2.39 as green crosses and circles, respectively. We observe that the linear fit of the simulated measurements $\left|S_{43}\right|-\left|S_{23}\right|$ and $\left|S_{41}\right|-\left|S_{21}\right|$ are also symmetric in this case. Therefore, $Y_{p 13}=0$, even with 1 transition bad coupled to the middle cell.

Finally, the simulations were repeated when breaking all symmetries in the cell. In this way, the position of taper 3 was kept at $+1 \mathrm{~mm}$ in $Z$, taper 2 change to $+1 \mathrm{~mm}$ in the $X$ axis and the other tapers remain well coupled. The resulting $S$ parameters are plotted in Figure 2.39 in red, where crosses represent $\left|S_{41}\right|-\left|S_{21}\right|$ and circles correspond to $\left|S_{43}\right|-$ 
$\left|S_{23}\right|$. In this case, both linear fit cross the horizontal axis very close to zero. However, a deviation of the calculated EM centre $Y_{p 13}$ with respect to the geometric center has a value of $1.5 \mu \mathrm{m}$.

From these results, we can conclude that the coupling between the four damping waveguides of the middle cell and the four tapered transitions affect the position measured for the EM axes. We strive for the reliability of the experimental measurements, so we worked to achieve a mechanical coupling in all tapered transitions lower than $1 \mathrm{~mm}$ to ensure a theoretical accuracy of less than a micron, which is not mechanically challenging.

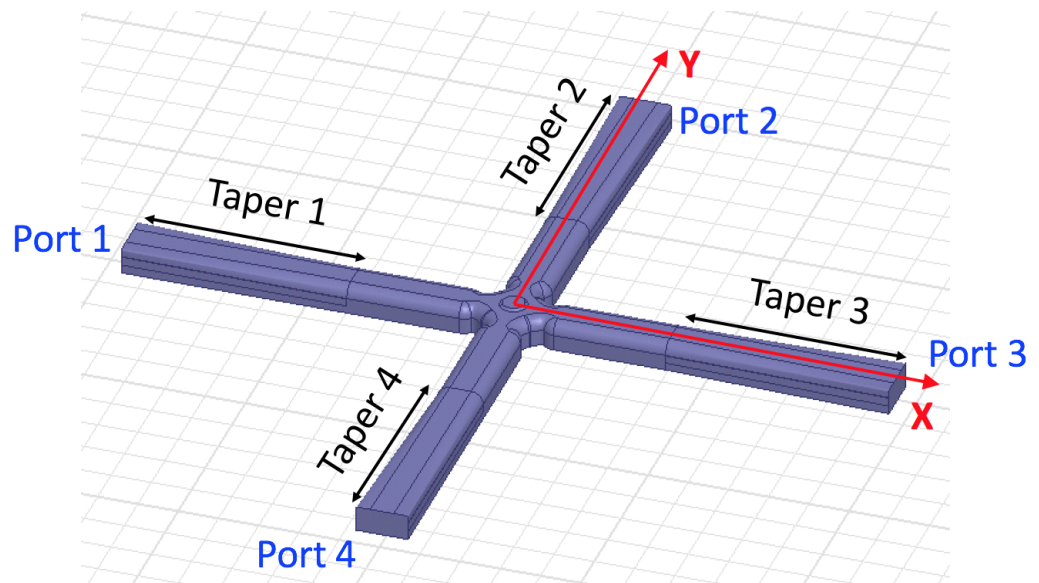

Figure 2.38: Four tapered transitions coupled to the middle cell of the TD24 at the end of the four damping waveguides. 


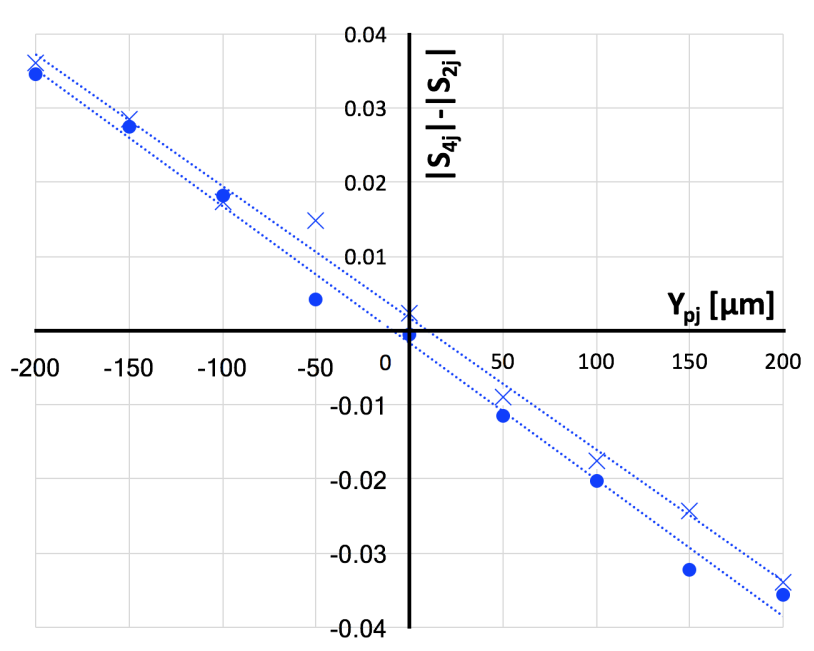

(a)

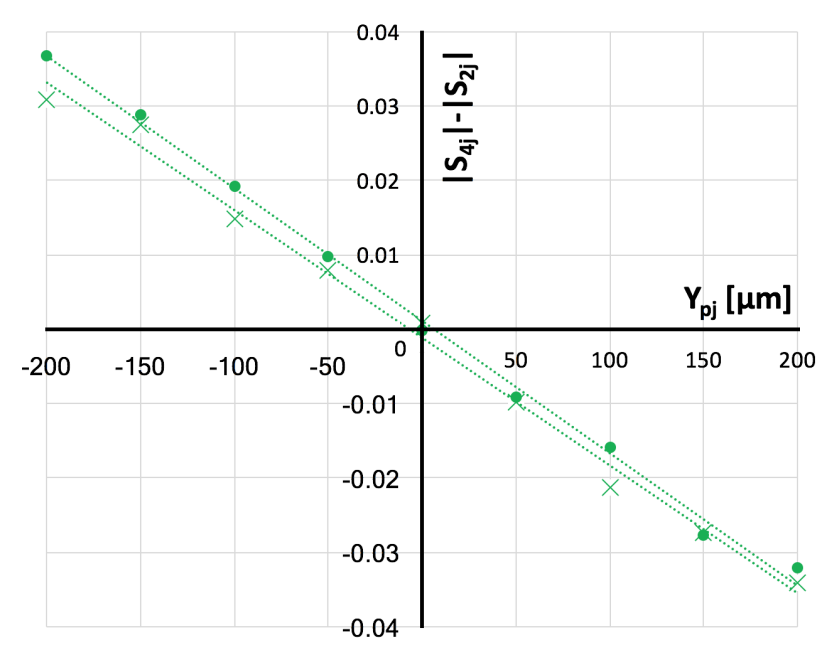

(b)

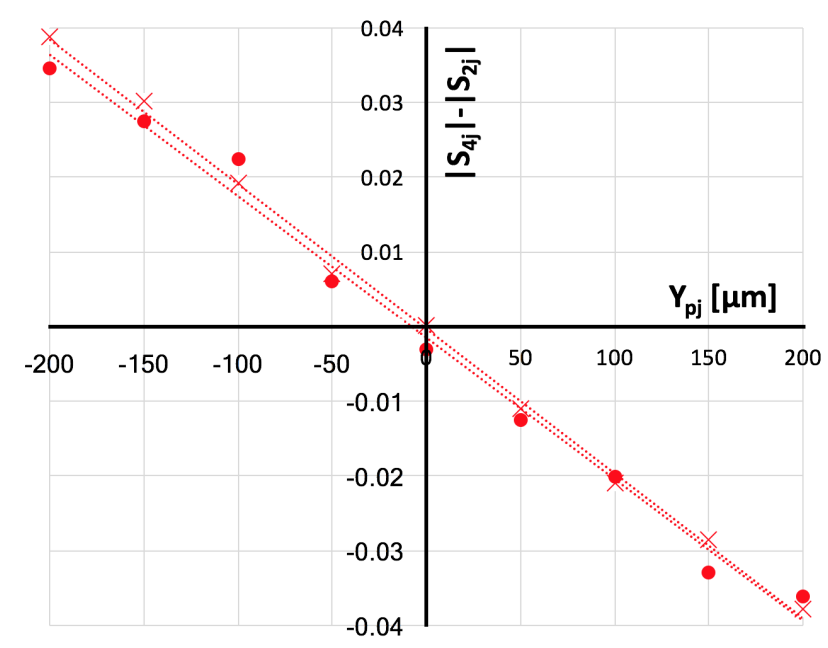

(c)

Figure 2.39: (a) $\left|S_{41}\right|-\left|S_{21}\right|$ (crosses) and $\left|S_{43}\right|-\left|S_{23}\right|$ (circles) when (a) there is a perfect coupling between tapers and damping waveguides. (b) the position of taper 3 is $\Delta \mathrm{Z}=+1 \mathrm{~mm}$, while the other tapers are perfectly coupled to their corresponding damping waveguides. (c) taper 3 is shifted $\Delta \mathrm{Z}=+1 \mathrm{~mm}$, taper 2 is shifted $\Delta \mathrm{X}=+1 \mathrm{~mm}$ in $X$ and the other tapers are well matched with the damping waveguides. 


\subsubsection{Impact study of the material and wire radius}

Up to now, all the simulation studies have been performed using a pure copper wire as a good approximation of the PACMAN wire. Finally, for completeness of this study, we performed simulation measurements of the $S$ parameters using different wire materials and radius.

We consider the smallest dielectric and conductor available wires in the market, given the small aperture of the AS. Polyamide and copper-beryllium wires were found, with radius $250 \mu \mathrm{m}, 125 \mu \mathrm{m}, 50 \mu \mathrm{m}$ and $25 \mu \mathrm{m}$. We aim to maximise sensitivity with respect to the wire position inside the middle cell of the AS in HFSS in order to gain resolution. The simulated model consists in one single cell -the middle one- and a wire moving along $4 \mathrm{~mm}$ in steps of $500 \mu \mathrm{m}$ along $Y$, while $X$ remains fix. The $S$ parameters when a polyamide wire with a radius of $125 \mu \mathrm{m}$ is inserted in the cell, are obtained at port 1 and can be seen in Figure 2.40. As observed, neither of the S-parameters are sensitive to the wire displacements inside the AS. This result is expected from Eq (2.41) given the small radius of the dielectric wire and this is the reason why a very thin nylon wire is used to allocate a small spheric bead in most of the cases where the bead-pull technique is applied. These simulations were reproduced for dielectric wires of different radius, delivering the same results as Figure 2.40.

We repeated the same simulations with a wire made of copper. In this case, the measured $S$ parameters varied with the position and the wire radius as obtained from Figure 2.41. Notice that bigger radius lead to smaller sensitivities and resolutions at around the geometric centre. So, it seems that the selected wire at PACMAN is also a good option to detect the EM axis of the TD24.

In subsection 2.5.1 we found that $Y_{p 1}$ and $Y_{p 3}$ are symmetric from zero. In order to verify that this result comes from the wire radius, we performed a simulation in which a copper wire of $200 \mu \mathrm{m}$ of radius moved along $(X, Y)=(0,-200) \mu \mathrm{m}$ to $(X, Y)=(0,200) \mu \mathrm{m}$ in steps of $10 \mu \mathrm{m}$. The resulting $\left|S_{41}\right|-\left|S_{21}\right|$ and $\left|S_{43}\right|-\left|S_{23}\right|$ are plotted in Figure 2.42, following the regression lines in red and blue, respectively. We could observe, that $m_{y, p 1}=m_{y, p 3}$. However, $a_{y, p 1}$ and $a_{y, p 3}$ do not have a value symmetric from zero. So, when calculating $Y_{p 13}$, the position of the centre in $Y$ is deviated from zero. We could conclude, once more, that the PACMAN wire is a good compromise. 


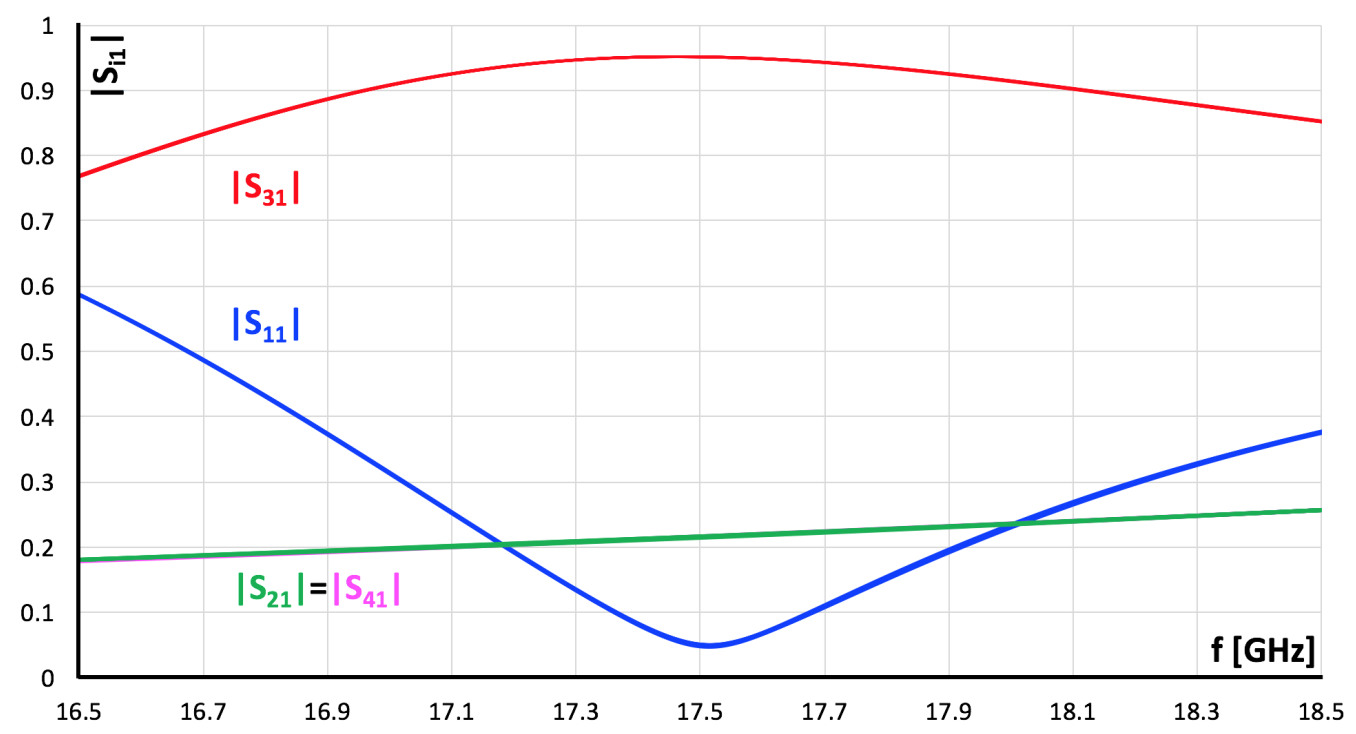

Figure 2.40: $\left|S_{11}\right|$ (f), $\left|S_{21}\right|$ (f), $\left|S_{31}\right|$ (f) and $\left|S_{41}\right|(\mathrm{f})$ of a polyamide wire with a radius of $125 \mu \mathrm{m}$ in the middle cell.

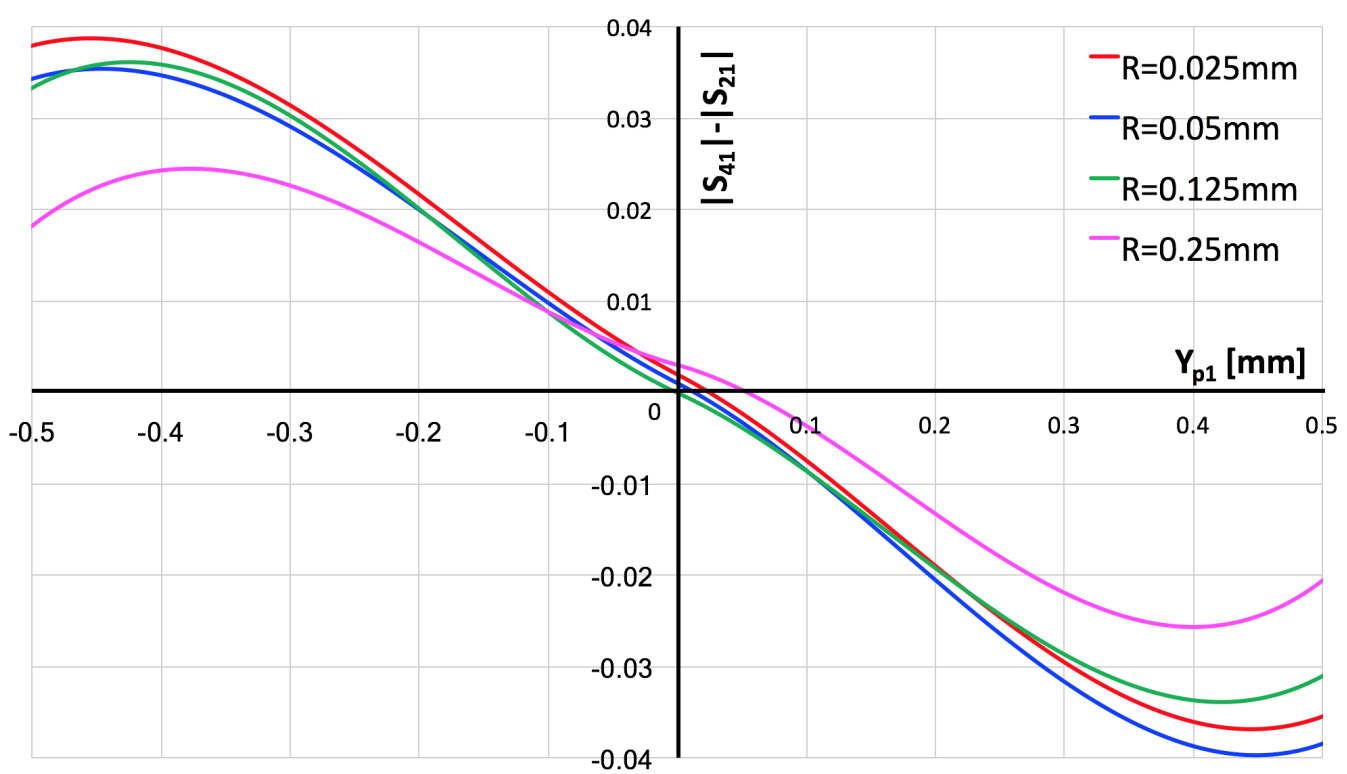

Figure 2.41: $\left|S_{41}\right|-\left|S_{21}\right|$ at $17 \mathrm{GHz}$ in the middle cell when a pure copper wire of different radius, $R$, moves in $Y$. 


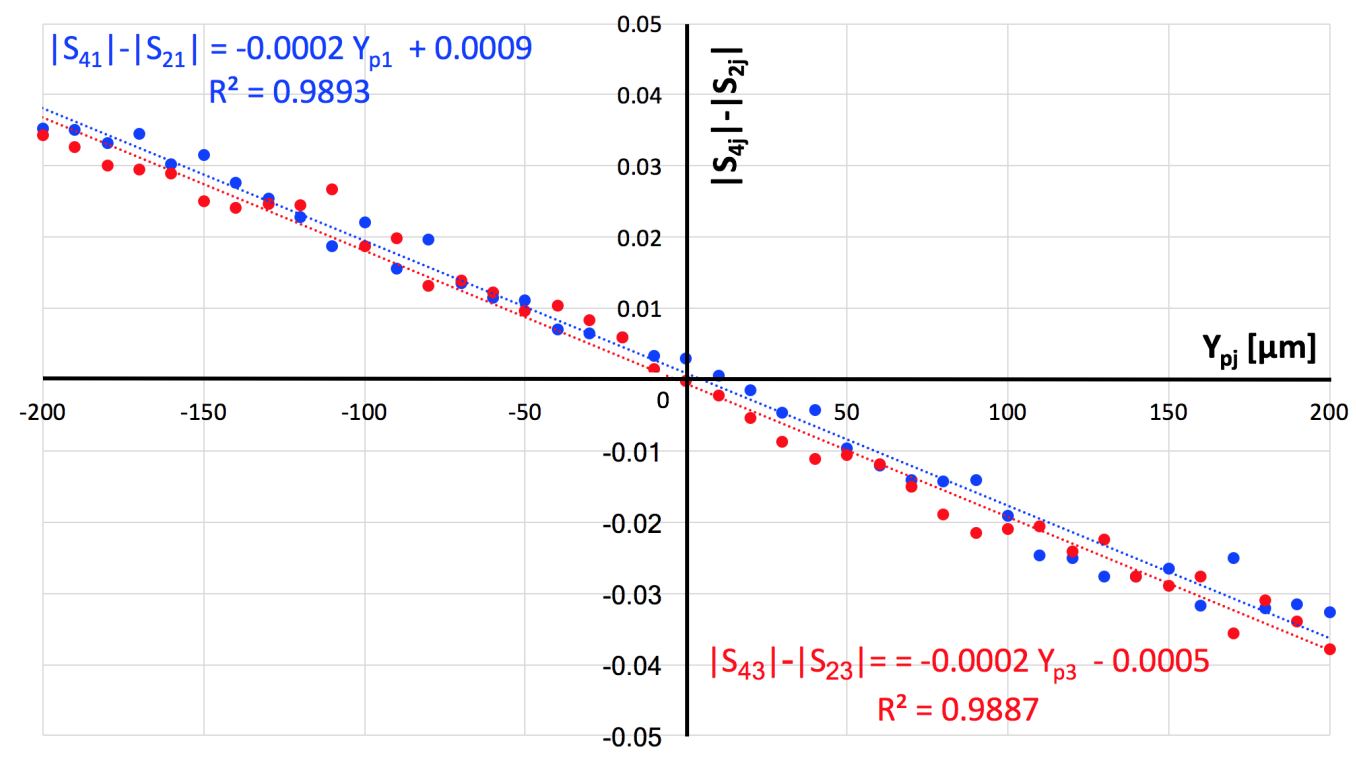

Figure 2.42: Simulated $\left|S_{4 j}\right|-\left|S_{2 j}\right|, j=1,3$, at $17 \mathrm{GHz}$ in the middle cell when a copper wire with a radius of $200 \mu \mathrm{m}$ moves along $Y$ and linear fit of the simulated asymmetry measurements. 


\section{Chapter 3}

\section{Design, fabrication and calibration of an experimental set-up to measure the EM axes of the CLIC TD24 using the perturbative method}

A test-bench has been developed within this Ph.D. thesis to measure the EM axis in the middle disk of the CLIC TD24 AS, see Figure 3.1, with the method proposed in Chapter 2. The design was inspired on a wire test set-up developed for BPM characterisation [79] at the Instituto de Física Corpuscular (IFIC) in Valencia, Spain.

The simplest configuration conceived, given the length of the structure $(341.1 \mathrm{~mm})$ and its mean iris aperture diameter $(5.5 \mathrm{~mm})$, consists on a vertical assembly of the elements as shown in Figure 3.2. The main advantages of such disposition are the elimination of sag in the wire, and the easy introduction of the wire with gravity. Indeed, the installation of the wire through the length of the structure without touching the walls is a delicate operation.

In the following sections of this chapter, a detailed description of the test bench elements, calibrations for the minimisation of errors, and the automatisation of the measurement process through the software implementation of the measurement algorithm will be explained.

\subsection{Design of the test-bench}

The main objective of this work is to measure the EM centre in the middle cell of the CLIC TD24 AS, whose position is defined in two planes: the horizontal ( $X$ axis) and the vertical ( $Y$ axis), as shown in Figure 3.2. Thus, the accelerating structure is moved in two degrees of freedom orthogonal to each other while the wire is fixed. A third degree of freedom $(\varphi)$ is introduced in order to rotate the structure around the wire direction $(Z$ axis). However, this was not used as the movement of the VNA cables would be too large 


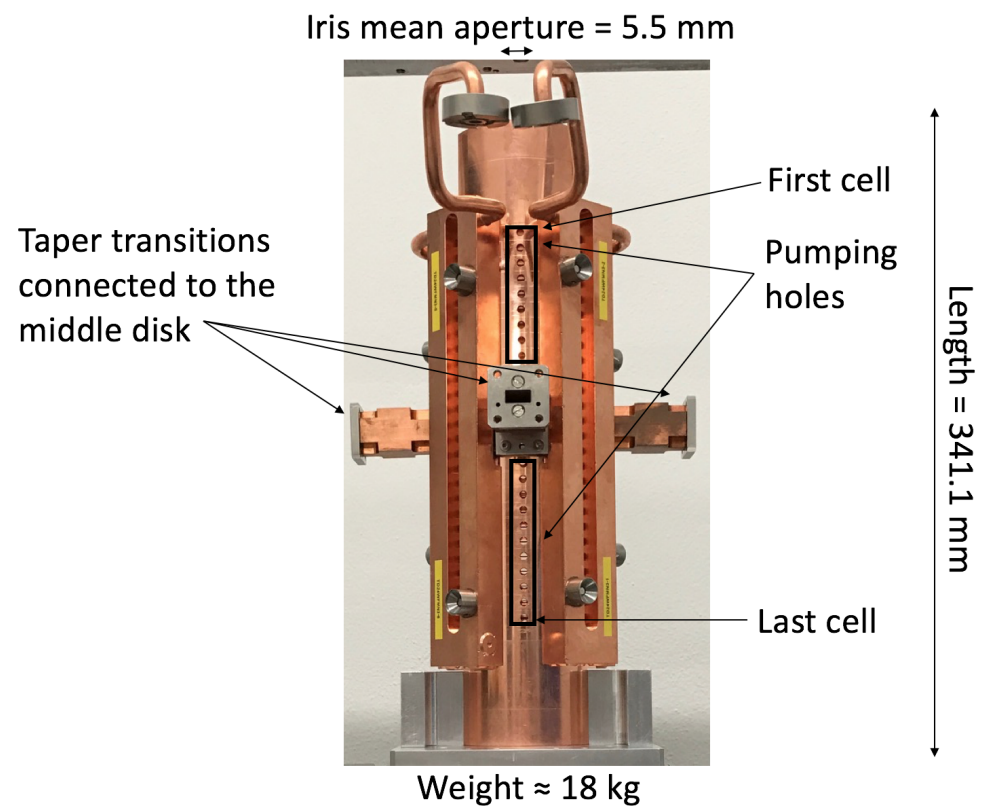

Figure 3.1: The TD24 AS and the accessibility to the cells.

to be compatible with the measurement accuracy requested. The pitch and yaw axes are fixed.

The 3D design of the test bench presented in Figure 3.2 was done using Computer-Aided Three Dimensional Interactive Application (CATIA), and it was registered at the CERN Engineering and Equipment Data Management Service (EDMS) under document number CLIASTND0159 [83]. A picture of the test set-up is shown in Figure 3.4. The test bench stands on a heavy rectified aluminium base where two linear stages are installed orthogonally to allow the vertical and horizontal movement of the AS relative to the wire $(x, y)$. On top of them, an aluminium case hosting a rotation stage is mounted to rotate the AS with respect to the wire $(\varphi)$. The case allows the free motion in both vertical and horizontal directions. The DC motors of the three stages are driven by a motion controller, all from Newport and provided by IFIC for the development of this work. The model of the translation stages is the ILS100CCHA [84], the URS150BCC [85] for the rotation stage and the ESP300 [86] for the controller. The maximum linear travel range of the translation stages is $100 \mathrm{~mm}$ with an accuracy of $4 \mu \mathrm{m}$. The rotation stage has an accuracy of $0.012^{\circ}$ in the bi-directional angular positioning and a resolution of $0.0005^{\circ}$. The encoder in the controller provides a resolution on the position of the translation stages of $0.1 \mu \mathrm{m}$ and $0.1 \mathrm{~m}^{\circ}$. The maximum normal load capacity of the stages is $25.5 \mathrm{~kg}$ and $30.6 \mathrm{~kg}$ for the translation and the rotation stages respectively. On top of the rotation stage, a support holding the AS vertically is placed. Taking into account that the accelerating structure is $17 \mathrm{~kg}$, the linear stage beneath the tower assembly stands a load capacity which is just at the limit of its functioning within the specifications.

The whole tower is surrounded by an aluminium frame in order to straighten the wire at both sides of the AS. On top of the frame, two linear stages -model M-UMR3.5 [88] from Newport- are mounted orthogonally, see Figure 3.5. They are used to set and calibrate the wire perpendicularity with respect to the base of the AS allowing a maximum 


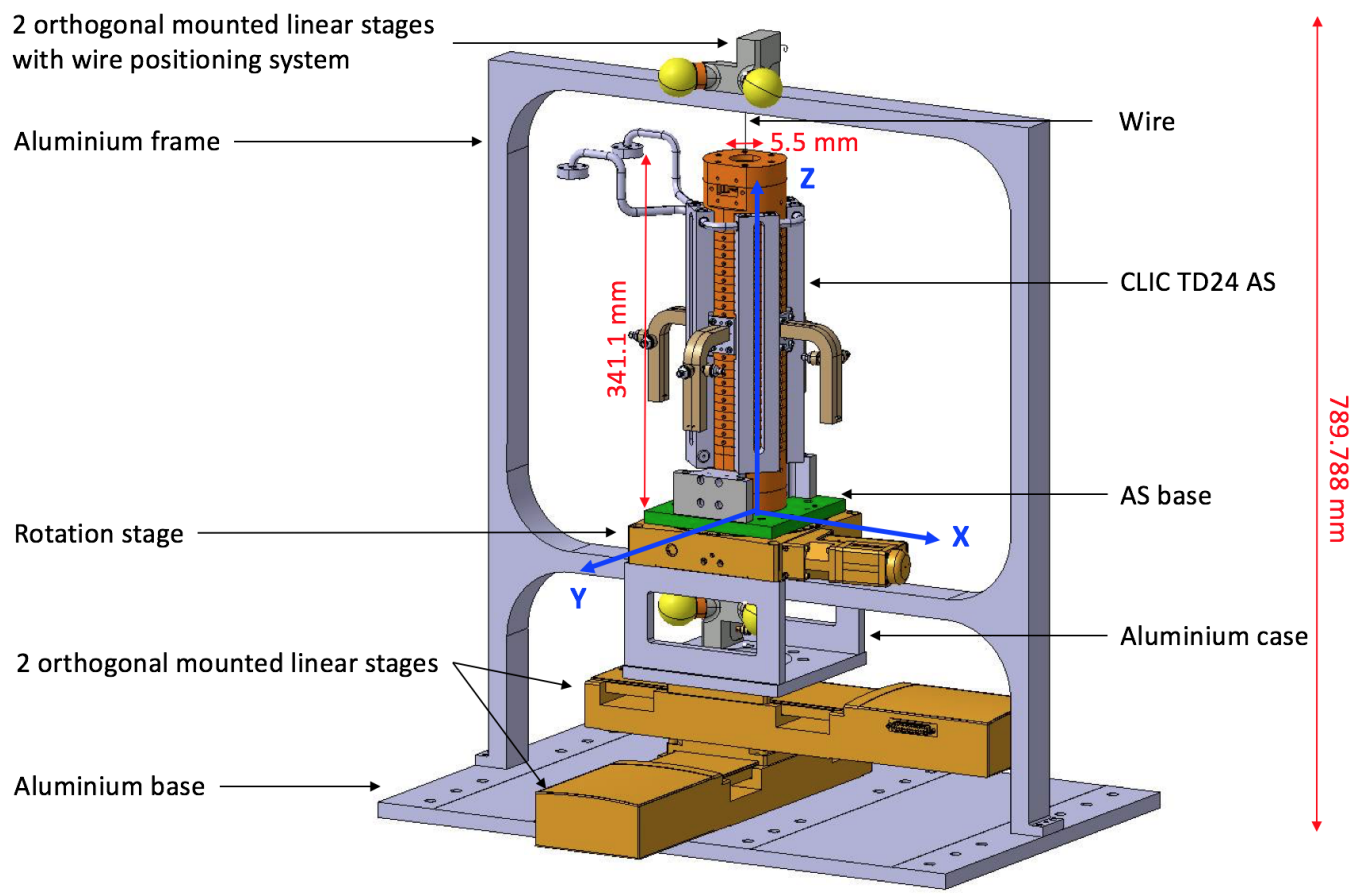

Figure 3.2: Design of the test set-up in CATIA, and coordinate system with the origin defined in the centre of the last cell of the AS.

stage travel of $5 \mathrm{~mm}$ in both $(x, y)$ directions. They serve also as the support of a wire positioning system as shown in Figure 3.5. The wire is fixed at this point until the bottom of the frame, passing just inside the aluminium case. Thanks to this case, the passage of the wire through the AS to the rotation stage is guaranteed, and it is fixed and stretched in another wire positioning system with a specification repeatability of $1.5 \mu \mathrm{m}$. Both systems were registered under EDMS numbers CLIASTND0158 and CLIASTND0161.

Finally, the whole set-up is settled in a granite vibration-damped table in order to minimise the wire vibrations during the measurements. The test-bench is localised in the grey room of building 162 at CERN with a constant temperature of $20 \pm 0.1^{\circ}$. The temperature is measured by a temperature station with a resolution of $0.1^{\circ}$ at a frequency of one minute. The model chosen was the 174T from TESTO [87].

A total of 18 spheric targets or fiducials -blue dots in Figure 3.4- are distributed around the bench for fiducialization purposes. The two wire positioning systems at each extreme of the wire are equipped with three fiducials each used to determine the absolute wire position. This reference is supported by a plane formed by 4 spheres placed on the frame of the test-bench. Thus, these 10 spheres form a reference system. The position of the AS is determined by measuring the position of 8 fiducials glued on its surface, forming a second reference system. This way, the relative position of the structure to the wire can be determined through the measurement of the 18 targets.

The selection of the wire has been explained in detail in Chapter 2, and is based on an investigation of different specifications using simulations in HFSS. A wire made of $98 \%$ copper and $2 \%$ beryllium with a diameter of $0.1 \mathrm{~mm}$ was purchased to THE GOODFEL- 
Chapter 3: Design, fabrication and calibration of an experimental set-up to measure the

LOW company [89].

To perform an $S$-parameter measurement, as explained in Chapter 2, a four-port Vector Network Analyzer (VNA) ZVA24 [90] from Rhode\&Schwarz is used. All ports of the VNA accept high frequency wires ended on SubMiniature version A (SMA) connectors screwed to SMA-WR51 transitions. Their plugging to the middle cell of the TD24 was done through taper transitions, as shown in Figure 3.1, made of copper allowing the symmetric geometry connections of the device under test. Their design was detailed in Chapter 2. Four pieces were manufactured at the CERN workshop, where the cost of the machining process used during fabrication was optimised. One of the resulting products is shown from different views in Figure 3.3.

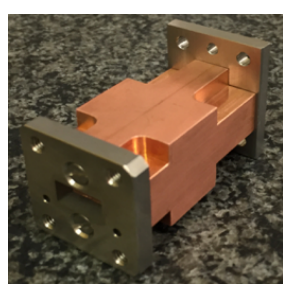

(a)

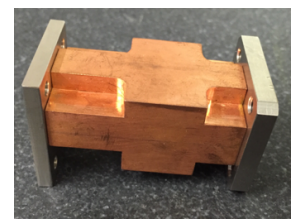

(b)

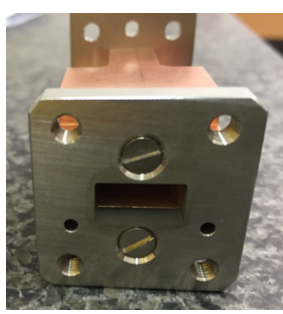

(c)

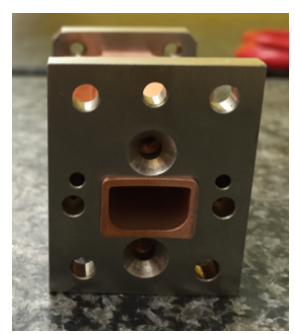

(d)

Figure 3.3: The manufactured taper transition from different views: (a) isometric projection, (b) right side view, (c) front view with WR-51 section and (d) back view with middle cell section.

A development environment has been programmed using the system-design platform called Laboratory Virtual Instrument Engineering Workbench (LabVIEW) [91]. It consists on a visual programming language from National Instruments (NI) that controls the stages and the VNA, and acquires and treats the data for completely automatized measurements. The VNA, the controller and the laptop are linked in series configuration through their GPIB ports. The standard from NI number 488.2 was used as interface cable GPIB to USB to allow the connection with the laptop. The algorithm has been constantly developed and optimised and will be explained in detail later in this chapter.

\subsection{Calibration of the experimental set-up and error estima- tion}

This section describes the process of error minimisation done by the calibration of the elements conforming the test bench. This process has been done in two steps: first, calibrations on the pyramidal integration of the components of the test bench, that is only done once; and second, calibrations on the instrumentation equipment required at every new set of measurements of the EM centre in the TD24 AS. 


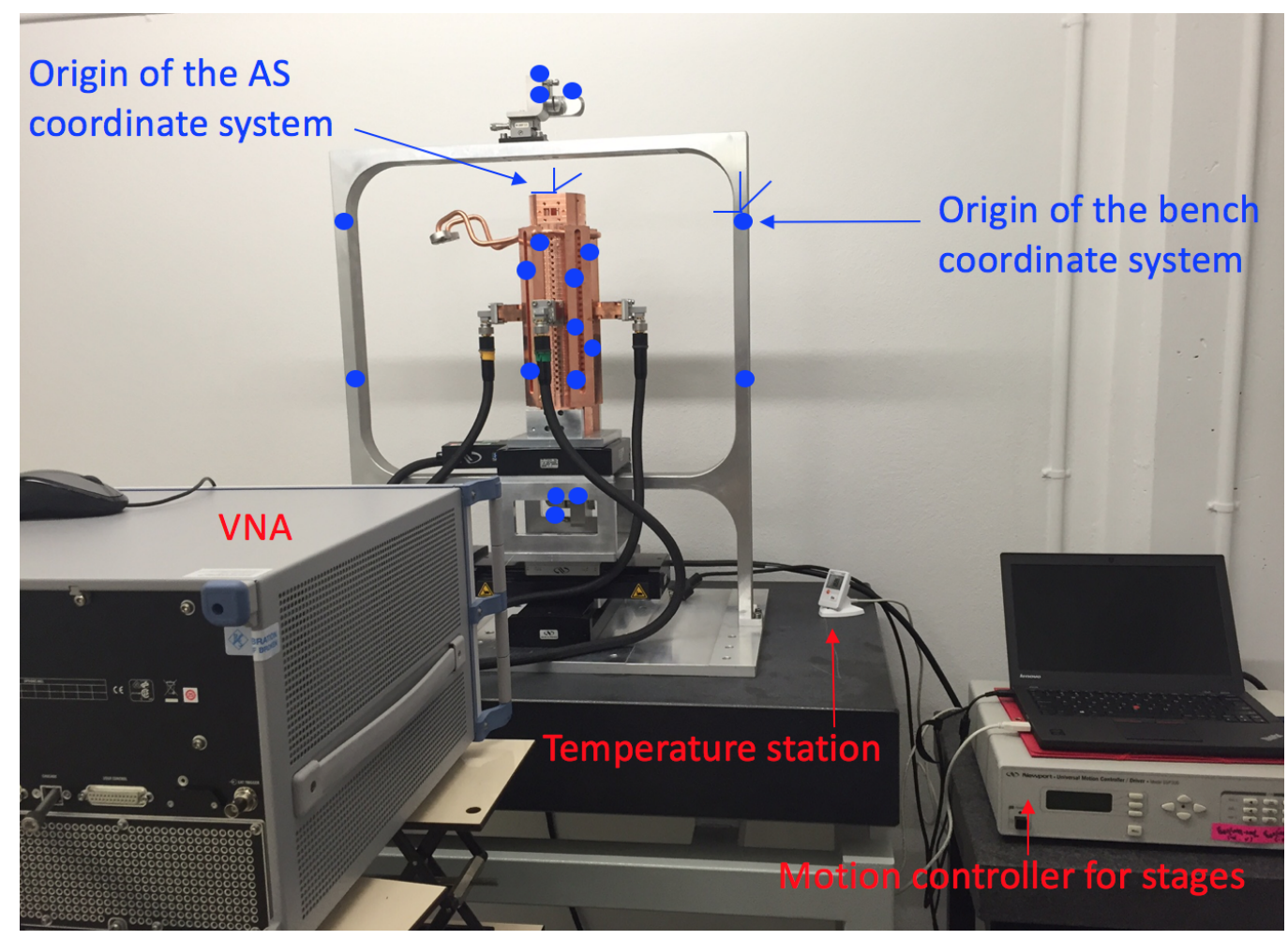

Figure 3.4: Experimental set-up with fiducials (blue dots) installed in the semi-clean room of building 162 at CERN.

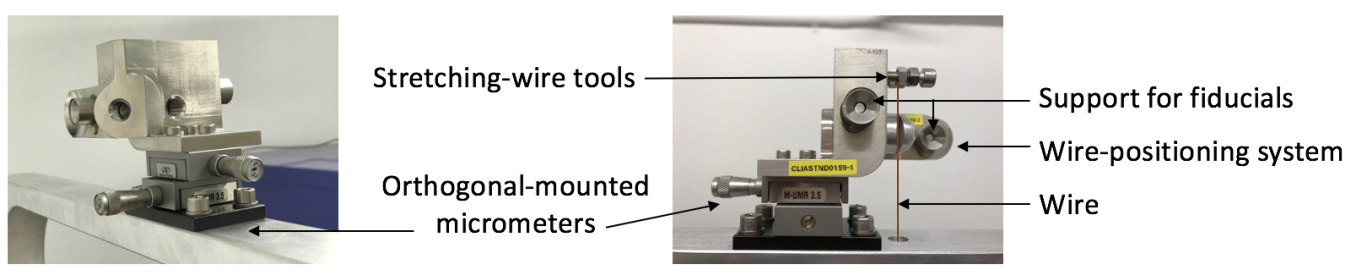

Figure 3.5: Two different views of the two orthogonal-mounted micrometers model MUMR3.5 from Newport and wire positioning system on top of them. 


\subsubsection{Calibration of the linear stages}

\subsubsection{Measurement set-up on the CMM}

The linear stages were mounted in their metallic rectified base, and the orthogonality was measured and corrected using the Leitz Infinity CMM 12.10.6. [93] at the metrology laboratory at CERN at the constant temperature of $20 \pm 0.1^{\circ}$.

The set-up is pictured in Figure 3.6, where both linear stages were mounted on the rectified aluminium base, and a sphere lying in a copper support was disposed in the middle of the platform that is moved by the linear stages. The sphere is a calibrated target measured by the CMM, whose position is calculated by measuring five points (red dots in Figure 3.7): one on top of the sphere and four around it. To perform this calibration, the stages were directly connected to the ESP300 controller, and this one to the laptop via GPIB-USB. A simple LabVIEW program was developed in order to move the stages to the desired position.

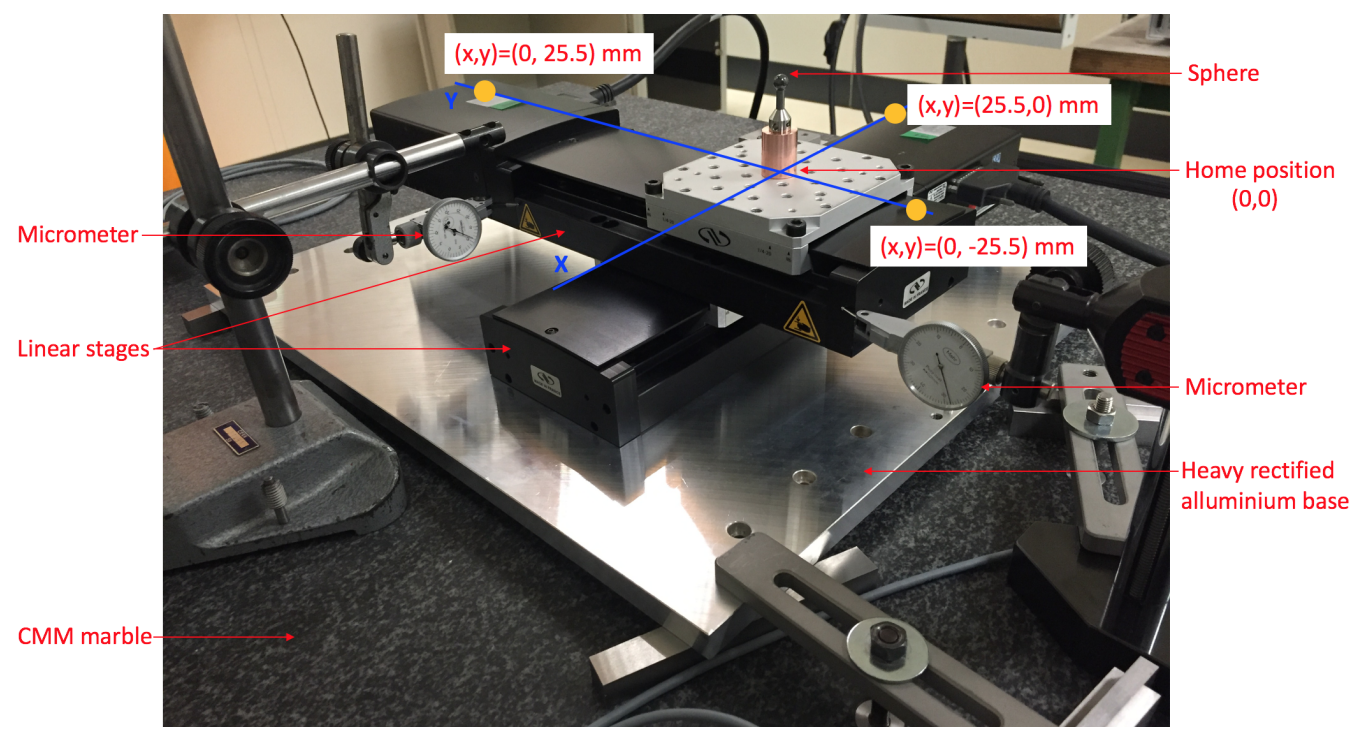

Figure 3.6: Set-up installed in the marble table of the Leitz Infinity CMM to calibrate the two linear stages. The yellow points represent the position of the sphere mounted in the movable platform.

\subsubsection{Correction of the orthogonality between the two linear stages}

This calibration was done bringing the sphere to the positions represented as yellow dots in Figures 3.6 and 3.7, and whose positioning follows the sequence indicated by the blue arrows. First, the platform containing the sphere was moved to the pre-defined home position at both axes $(X, Y)=(0,0)$. Then, the platform was shifted to the furthest extreme of one of the directions at $Y,(\mathrm{X}, \mathrm{Y})=(0,25.5) \mathrm{mm}$, and the position of the sphere was measured. After that, the sphere was driven to the other most extreme position on the opposite direction at $Y,(\mathrm{X}, \mathrm{Y})=(0,-25.5) \mathrm{mm}$. Once the new position was measured, the platform was moved to home position again to be later directed to one of the extremes 


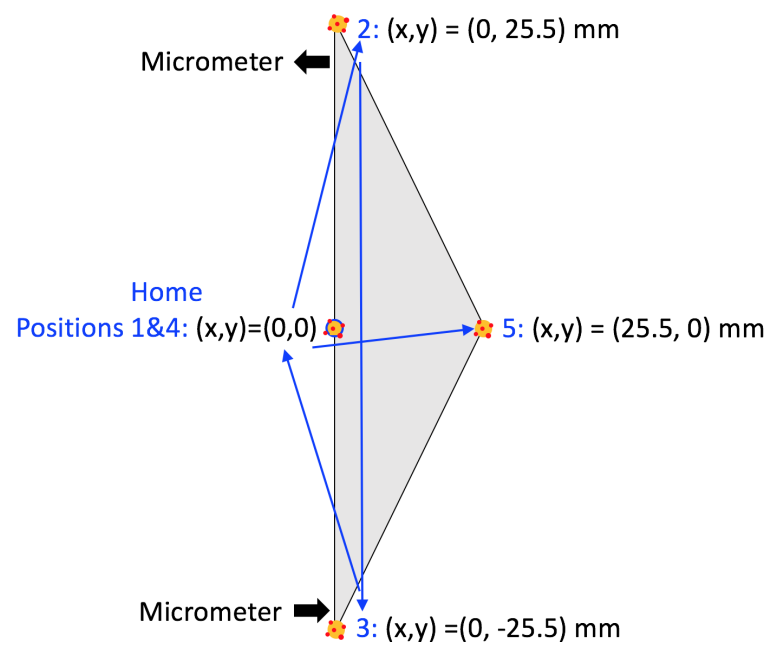

Figure 3.7: Measurement of the sphere at different positions for the correction of the orthogonality between the two linear stages. The arrows indicate the direction of motion of the platform moved by the stages through their travel to the different position numbers.

in $X,(\mathrm{X}, \mathrm{Y})=(25.5,0) \mathrm{mm}$. The link between the points indicated in Figure 3.7 gives a plane between $X$ and $Y$ that serves as a measurement of the orthogonality between both axes. A correction was applied to the $Y$ axis using a little hammer, in order to address it perpendicularly with respect to $X$, and this value was measured with two micrometers installed at both extremes of $Y$. This process was repeated several times until the achieved orthogonality was $13 \mu \mathrm{m}$ over a length of $51 \mathrm{~mm}$. This calibration was considered good enough for the maximum travel range of the accelerating structure without touching the wire, which is around $4 \mathrm{~mm}$. So, the corresponding orthogonality would be around $1 \mu \mathrm{m}$ around the diameter of the iris mean aperture.

\subsubsection{Error characterisation on the positioning of the linear stages}

Several measurements were done within the environment of the CMM in order to determine the uni-directional repeatability of the stages, typically defined as the ability of the instrument to achieve a given position from a single direction. The error, $\xi_{C M M}$, on the positioning of the linear stages was measured in a single direction in both $X$ and $Y$ when they move individually, and when they move at the same time. The difference between the position given to the linear stages through the controller and the position measured by the CMM in the different tests are compared in order to infer the mean error value. The measurements are numbered as follows:

- Test 1: $X$ move while $Y$ remains fixed to home position.

- Test 2: $Y$ move while $X$ remains fixed to home position.

- Test 3: $X Y$ move at the same time starting at $(\mathrm{X}, \mathrm{Y})=(-2,-2) \mathrm{mm}$, going to $(X, Y)=(2,2) \mathrm{mm}$ and back to $(X, Y)=(-2,-2) \mathrm{mm}$.

- Test 4: $X Y$ move at the same time starting at $(X, Y)=(-2,2) \mathrm{mm}$, going to $(X, Y)=(2,-2) \mathrm{mm}$ and back to $(X, Y)=(-2,2) \mathrm{mm}$. 
Chapter 3: Design, fabrication and calibration of an experimental set-up to measure the EM axes of the CLIC TD24 using the perturbative method

These tests are done withot a load in the moving platform, and are repeated later including a load of $25 \mathrm{~kg}$. The objective is to estimate the error on the positioning of the linear stages, $\xi_{C M M}$, as measured by the CMM when the AS is mounted on top of them in the test bench by loading the stages with $25 \mathrm{~kg}$.

The results concerning Test 1 without load are represented in Figure 3.8 (a). The plot shows that for each step, the mean $\xi_{C M M}$ increases as $0.4 \mu \mathrm{m}$ and it increases $0.3 \mu \mathrm{m}$ when going back to the previous position. The error bars in the plot represent the CMM uncertainty from $\mathrm{Eq}$ (2.1). The conclusion deduced from the results is that the accuracy measured is around the same as the uncertainty of the measuring instrument. The accuracy on the positioning on $Y$ when it is fixed to home position is also measured. The results are shown in Figure 3.8 (b). The mean value of all the measured errors is $-0.1971 \mu \mathrm{m}$ and the standard deviation is $0.3276 \mu \mathrm{m}$. This is also an estimation on the accuracy of the positioning on the stage in $Y$.

The error measurements, $\xi_{C M M}$, performed concerning Test 2 without load are plotted in Figure 3.9 (a). The results obtained in this test are better than the previous one: $\xi_{C M M}$ on each step has a mean error value of $0.11 \mu \mathrm{m}$, and it is $0.13 \mu \mathrm{m}$ on the way back to the previous point. These values are better than the uncertainty of the CMM from Eq (2.1), however it must be considered to be the same amount. $\xi_{C M M}$ of the $X$ plane, when it is required to be fixed at the home position, is also better, as demonstrated in Figure 3.9 (b). The mean value of the measured $\xi_{C M M}$ is $0.1794 \mu \mathrm{m}$ with a standard deviation of $0.1079 \mu \mathrm{m}$. Note that $\xi_{C M M}$ is bigger in $X$ than in $Y$, probably due to $Y$ being parallel to the CMM reference axis, and being $X$ slightly deviated from its orthogonal direction as measured before.

The error measurements, $\xi_{C M M}$, from Test 1 and Test 2 with a load of $25 \mathrm{~kg}$ are plotted in Figures 3.10 and 3.11. The results obtained are more promising than without a load, as summarised in Table 3.1. Note that the systematic error is smaller in $Y$ at home position in this case than in the previous test, and the other way around for $X$. However, both of them have lack of importance as they are systematic errors and therefore, can be cancelled from the measurement.

\begin{tabular}{|l|c|c|c|c|c|c|c|c|}
\hline & \multicolumn{2}{|c|}{ Test1 } & \multicolumn{2}{c|}{ Test2 } & \multicolumn{2}{c|}{ Test3 } & \multicolumn{2}{c|}{ Test4 } \\
\hline$\xi_{C M M}$ & no load & load & no load & load & no load & load & no load & load \\
\hline Step go X $[\mu \mathbf{m}]$ & 0.4 & 0.1 & - & - & 0.3 & 0.1 & 0.3 & 0.2 \\
\hline Step back X $[\mu \mathbf{m}]$ & 0.3 & 0.1 & - & - & 0.3 & 0.1 & 0.5 & 0.1 \\
\hline Step go Y $[\mu \mathbf{m}]$ & - & - & 0.1 & 0.1 & 0.4 & 0.2 & 0.4 & 0.2 \\
\hline Step back Y $[\mu \mathbf{m}]$ & - & - & 0.1 & 0.1 & 0.4 & 0.2 & 0.4 & 0.2 \\
\hline $\bar{X} @$ home $[\mu \mathbf{m}]$ & - & - & 0.2 & -0.6 & - & - & - & - \\
\hline$\sigma_{X} @$ @ome $[\mu \mathbf{m}]$ & - & - & 0.1 & 0.1 & - & - & - & - \\
\hline $\bar{Y} @$ home $[\mu \mathbf{m}]$ & -0.2 & -0.1 & - & - & - & - & - & - \\
\hline$\sigma_{Y} @$ home $[\mu \mathbf{m}]$ & 0.3 & 0.2 & - & - & - & - & - & - \\
\hline
\end{tabular}

Table 3.1: Error measured by the CMM, $\xi_{C M M}$, on the positioning of the linear stages with and without a load of $25 \mathrm{~kg}$. 
The performance of Test 3 and 4 without a load delivers the plots in Figures 3.12 and 3.13 , that can be compared with the corresponding ones with a load of $25 \mathbf{~ k g}$ presented in Figures 3.14 and 3.15. The results are also summarised in Table 3.1 and the conclusion that we can infer is that the linear stages have, in general, the same uncertainty in the positioning as the uncertainty of the most accurate measuring instrument available at CERN (Eq (2.1)).

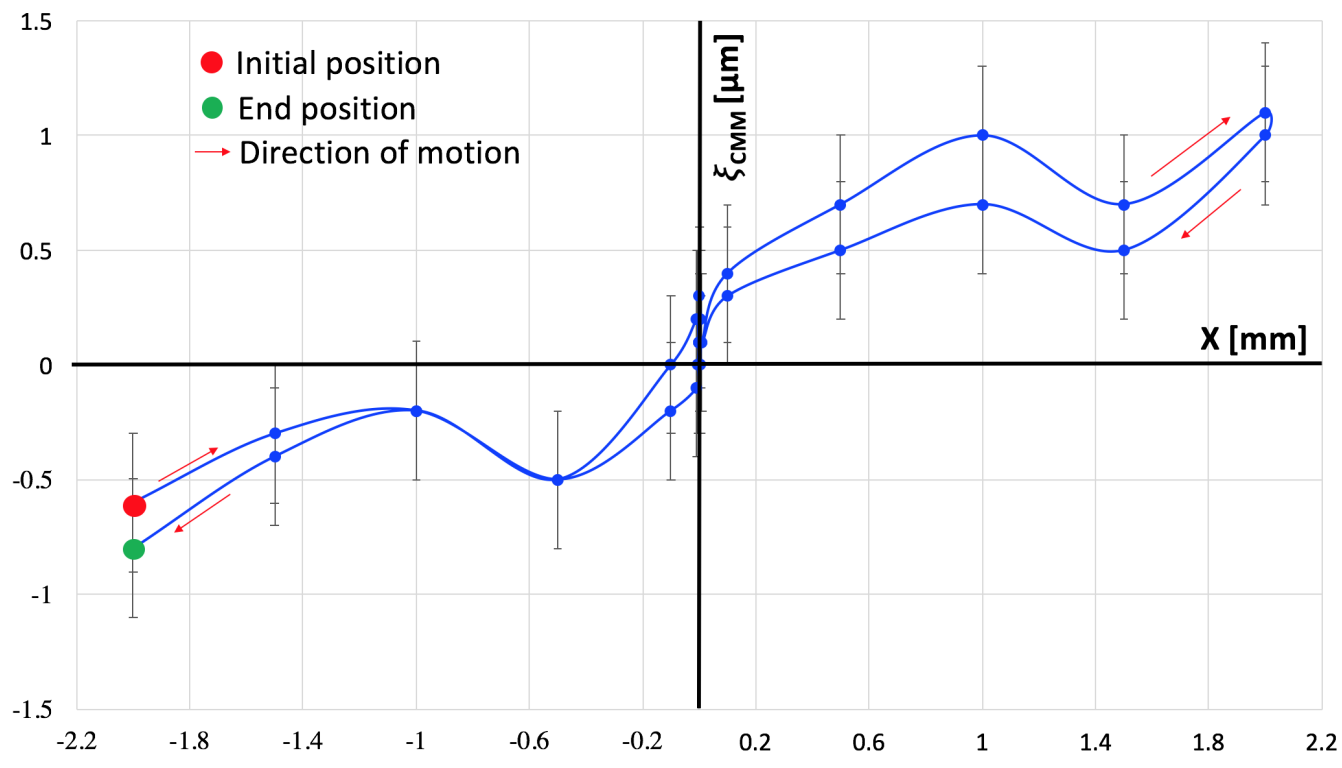

(a)

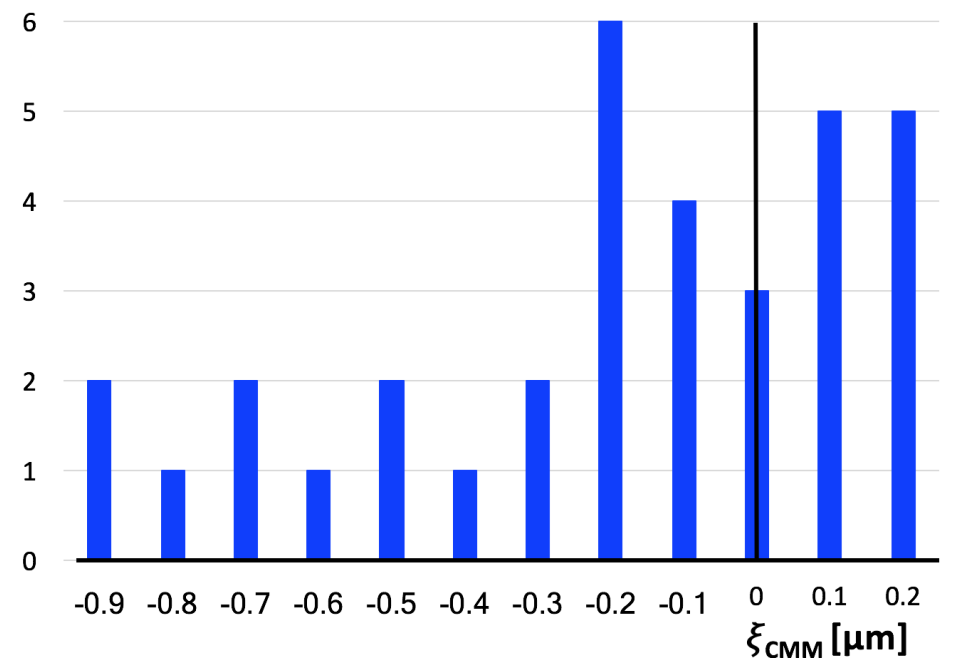

(b)

Figure 3.8: (a) Measurement of the accuracy of the linear stage in $X$. (b) Histogram of the measured error in $Y$ at home position. 
Chapter 3: Design, fabrication and calibration of an experimental set-up to measure the EM axes of the CLIC TD24 using the perturbative method

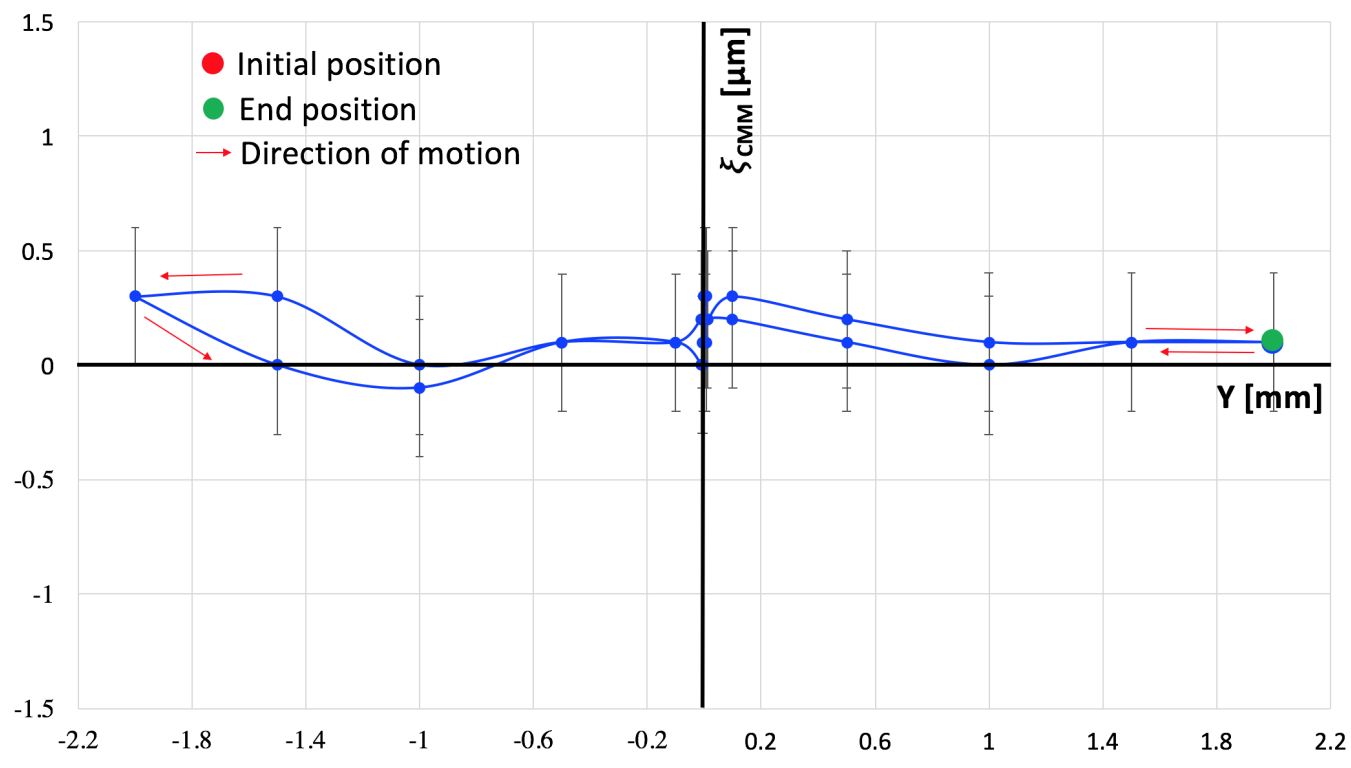

(a)

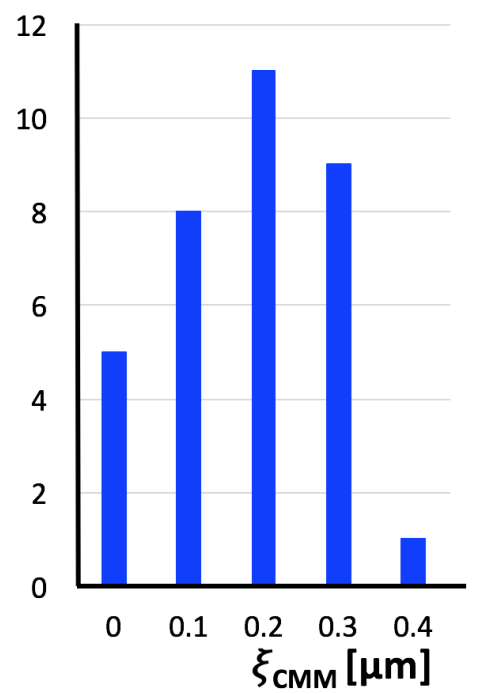

(b)

Figure 3.9: (a) Measurement of the accuracy of the linear stage in $Y$. (b) Histogram of the measured error in $X$ at home position. 


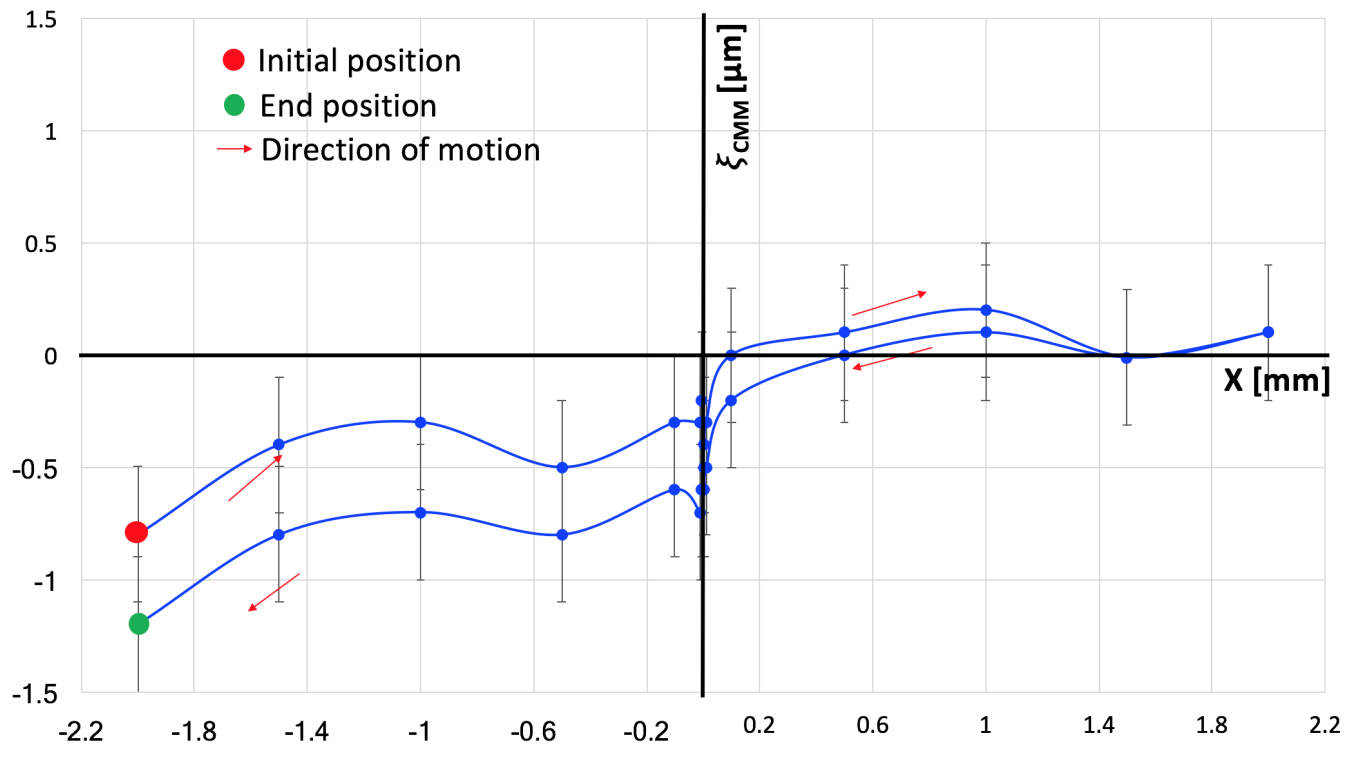

(a)

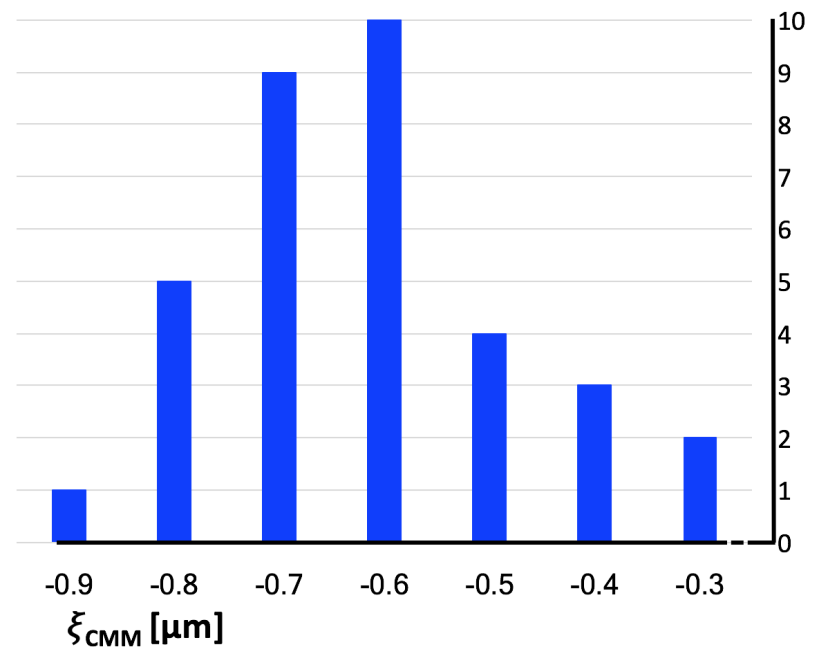

(b)

Figure 3.10: (a) Measurement of the accuracy of the linear stage in $X$ with a load of $25 \mathrm{~kg}$. (b) Histogram of the measured error in $Y$ at home position in (b). 
Chapter 3: Design, fabrication and calibration of an experimental set-up to measure the EM axes of the CLIC TD24 using the perturbative method

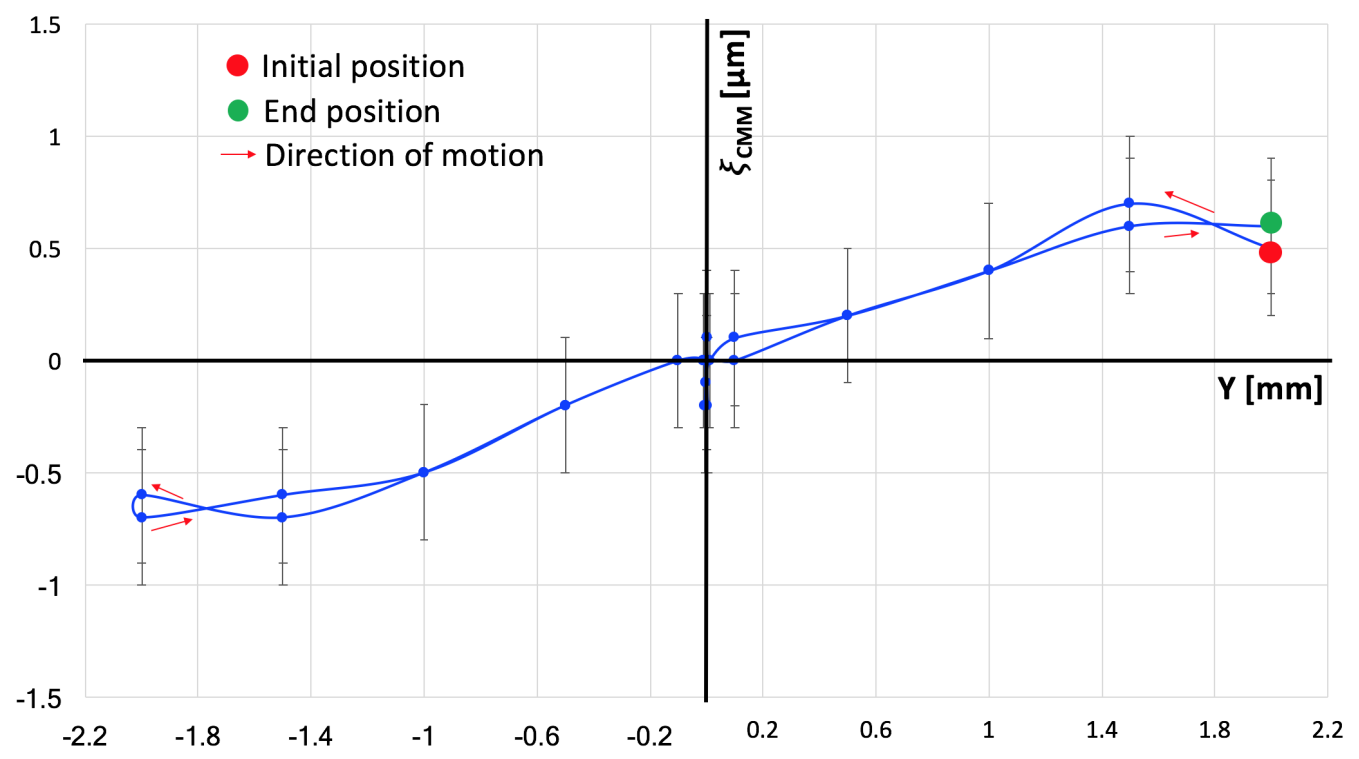

(a)

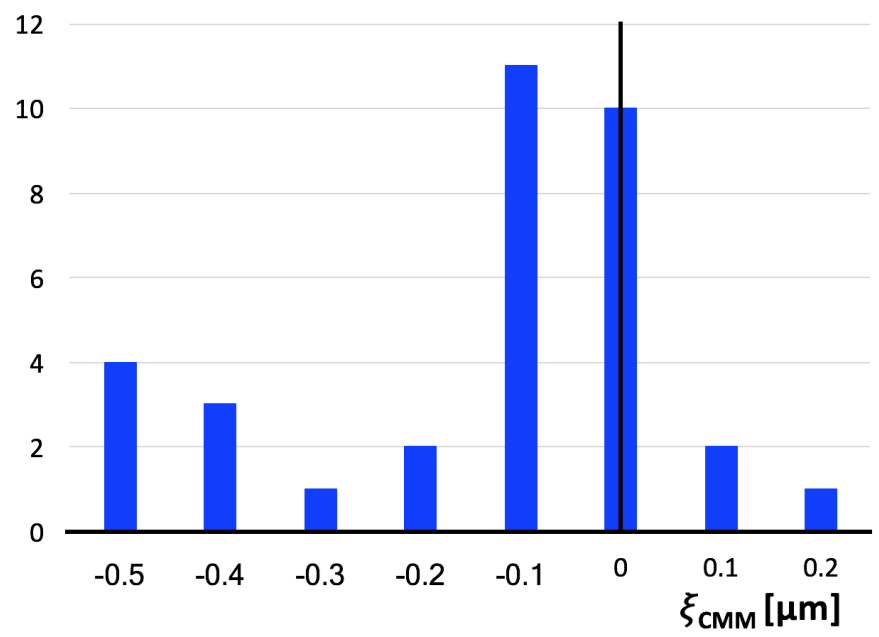

(b)

Figure 3.11: (a) Measurement of the accuracy of the linear stage in $Y$ with a load of $25 \mathrm{~kg}$. (b) Histogram of the measured error in $X$ at home position in (b). 


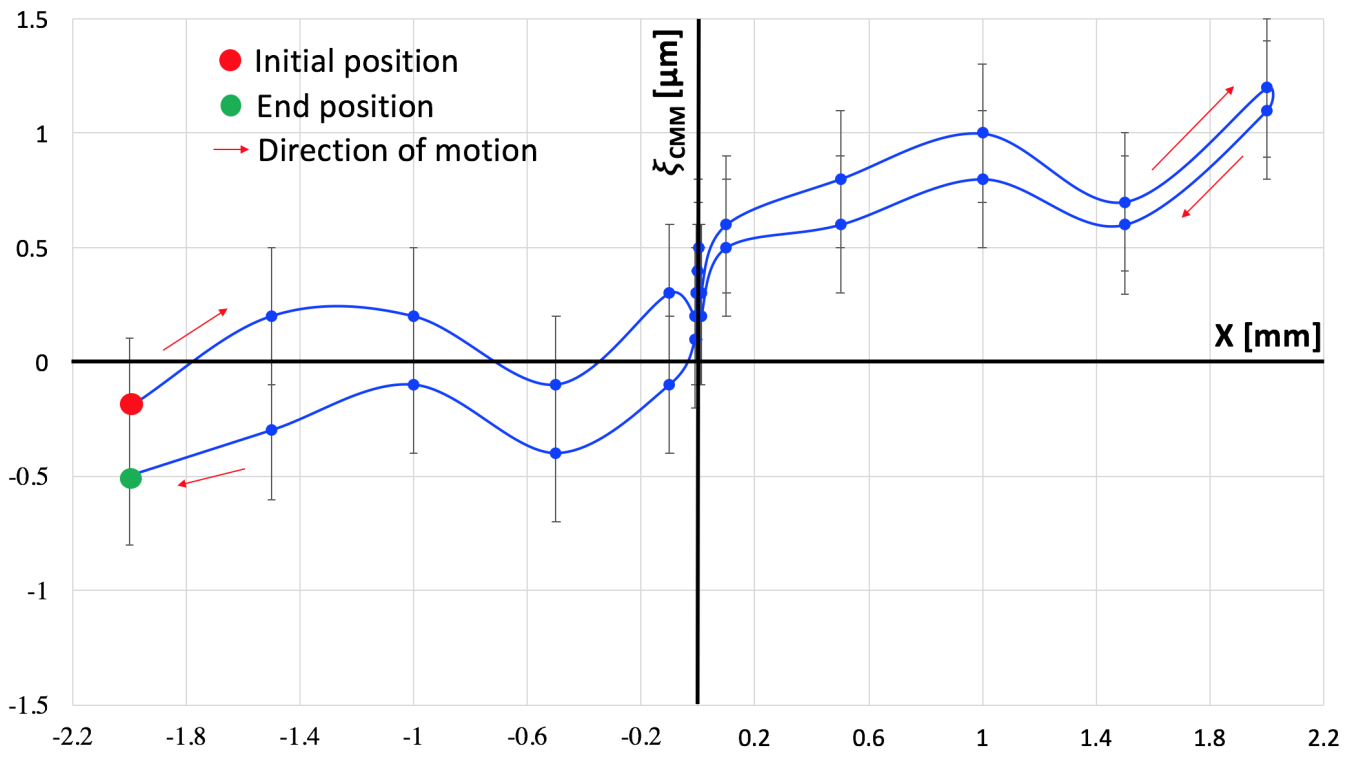

(a)

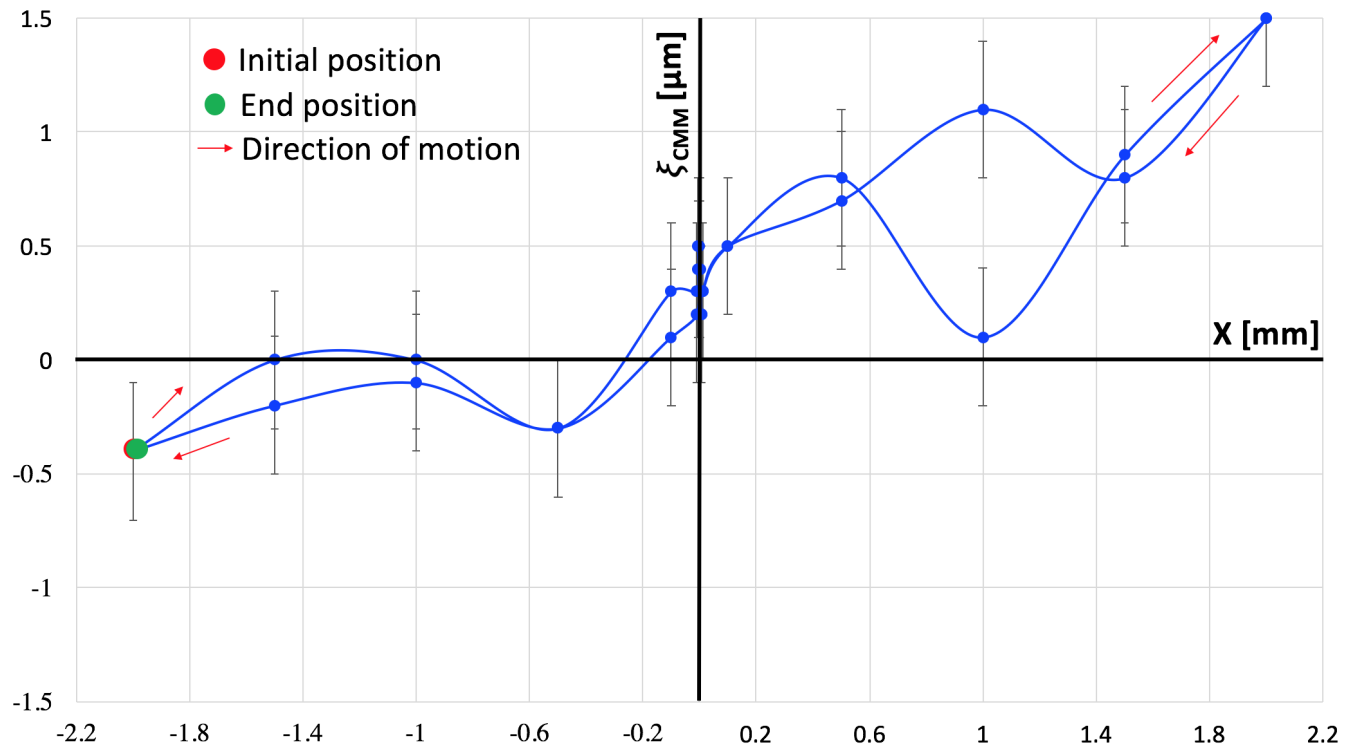

(b)

Figure 3.12: Measurement of the accuracy of $X$ without load when both linear stages are moved at the same time in (a) Test 3 and (b) Test 4. 
Chapter 3: Design, fabrication and calibration of an experimental set-up to measure the EM axes of the CLIC TD24 using the perturbative method

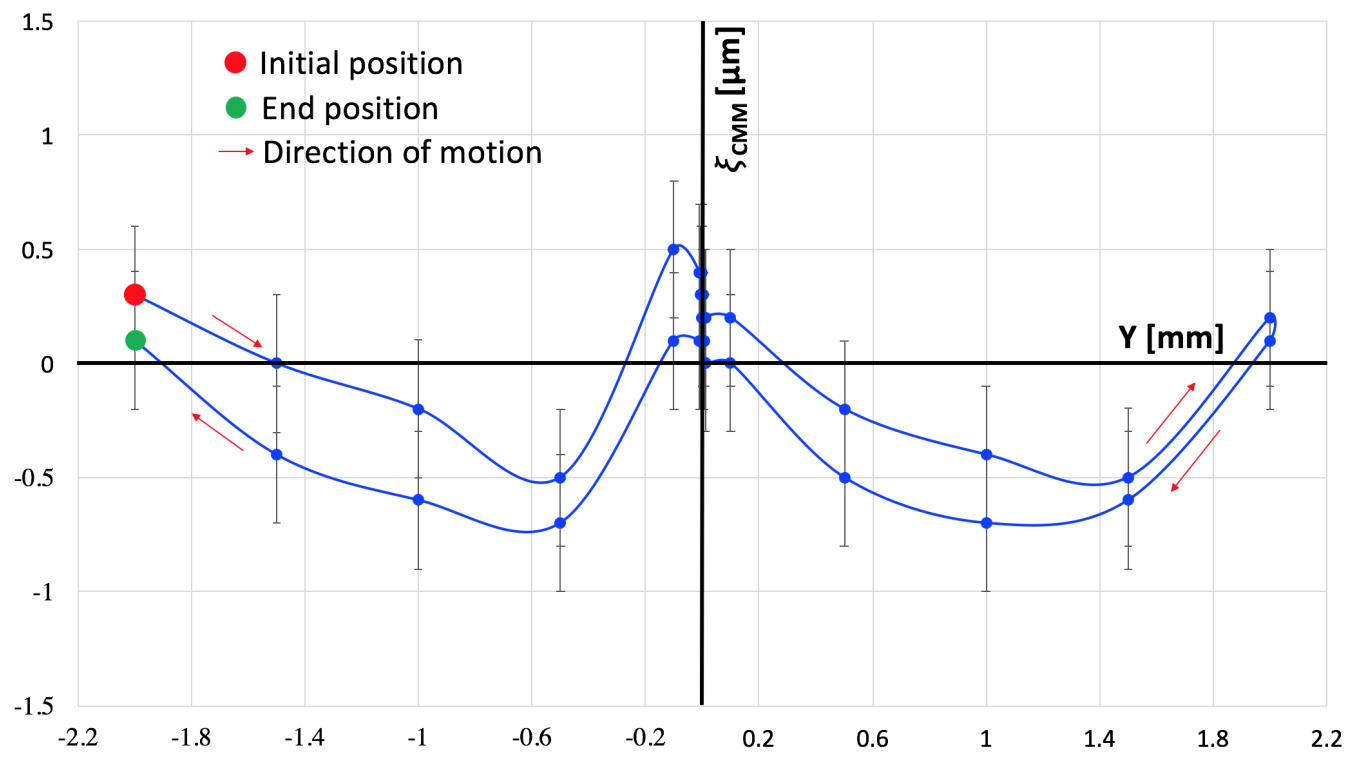

(a)

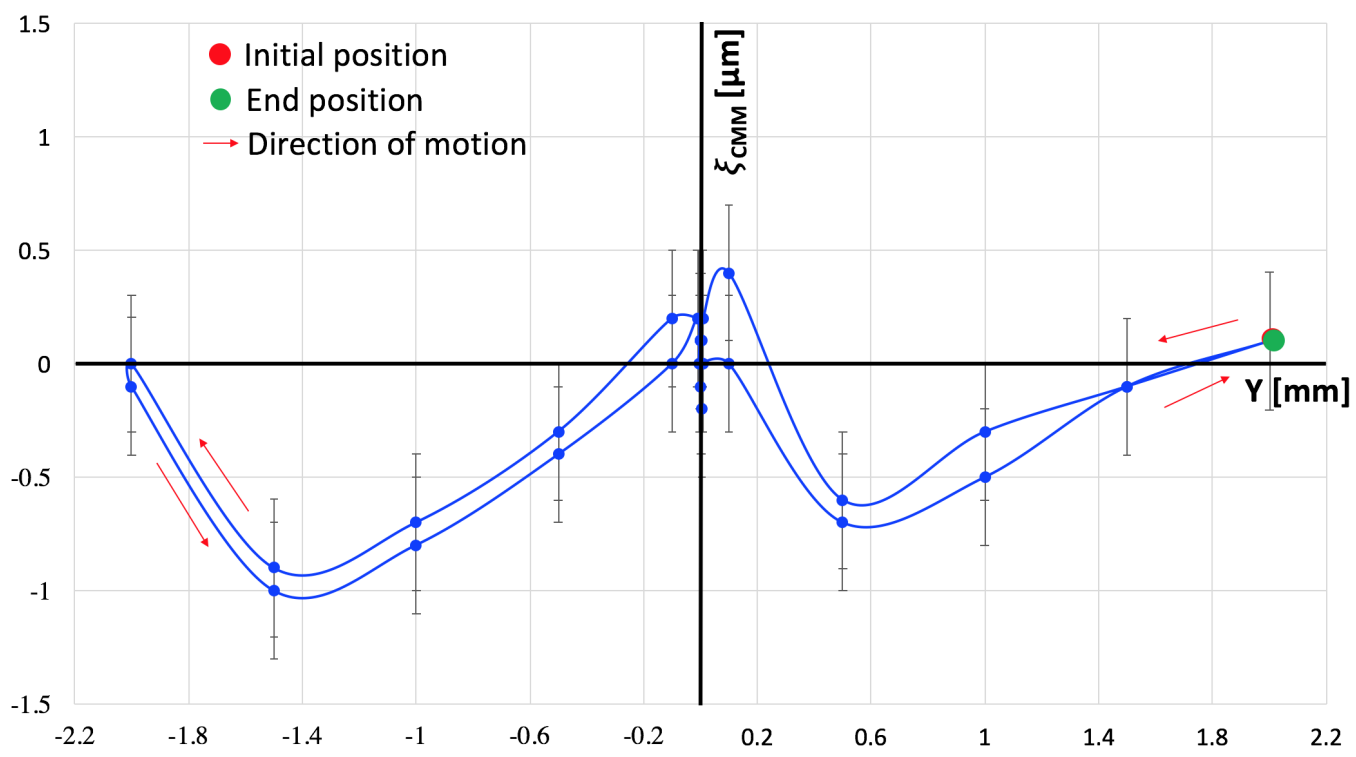

(b)

Figure 3.13: Measurement of the accuracy of $Y$ without load when both linear stages are moved at the same time in (a) Test 3 and (b) Test 4. 


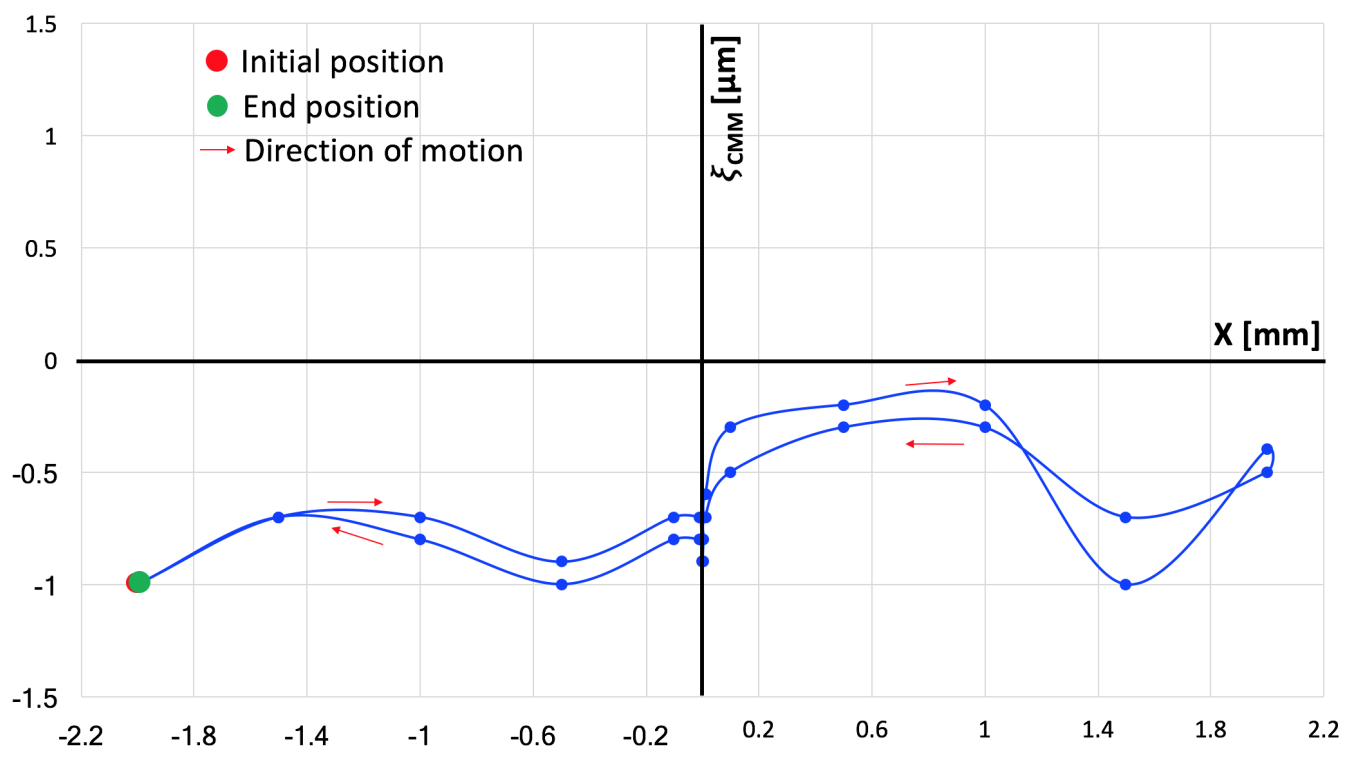

(a)

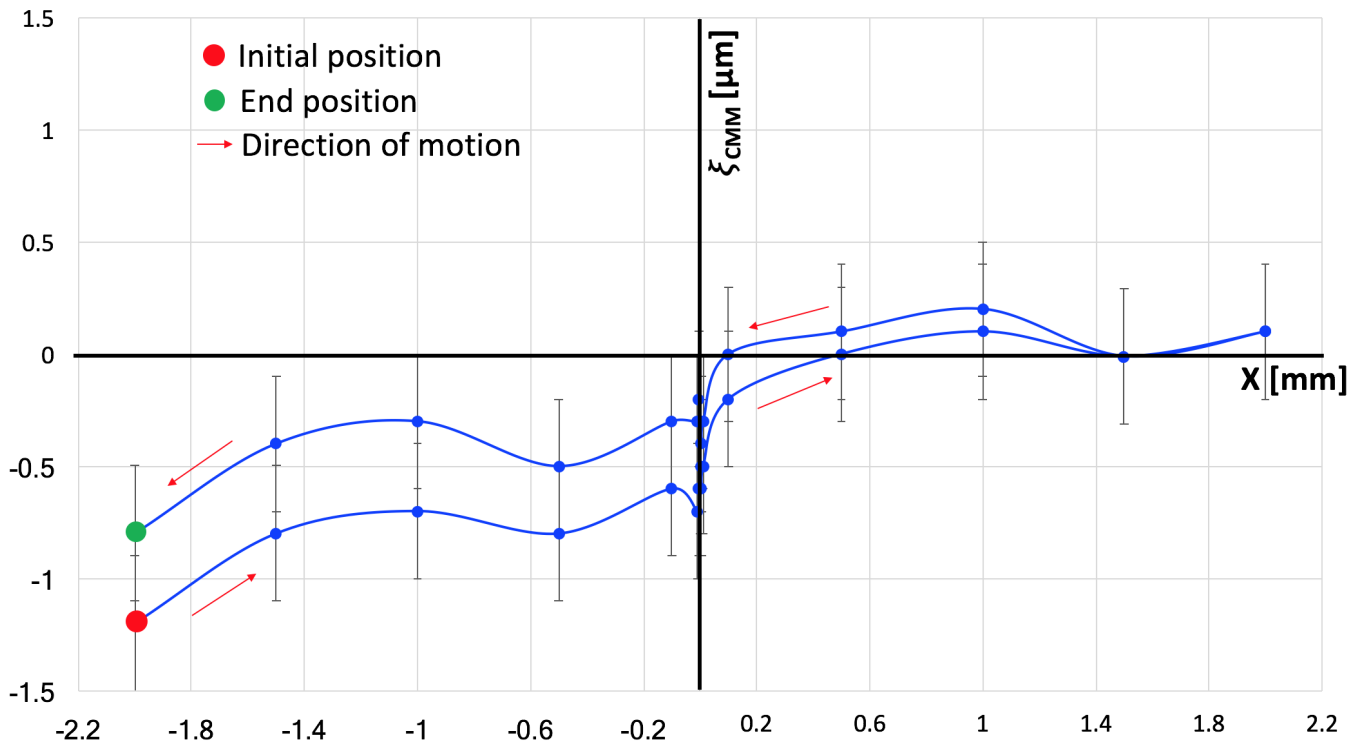

(b)

Figure 3.14: Measurement of the accuracy of $X$ with a load of $25 \mathrm{~kg}$ when both linear stages are moved at the same time in (a) Test 3 and (b) Test 4. 
Chapter 3: Design, fabrication and calibration of an experimental set-up to measure the EM axes of the CLIC TD24 using the perturbative method

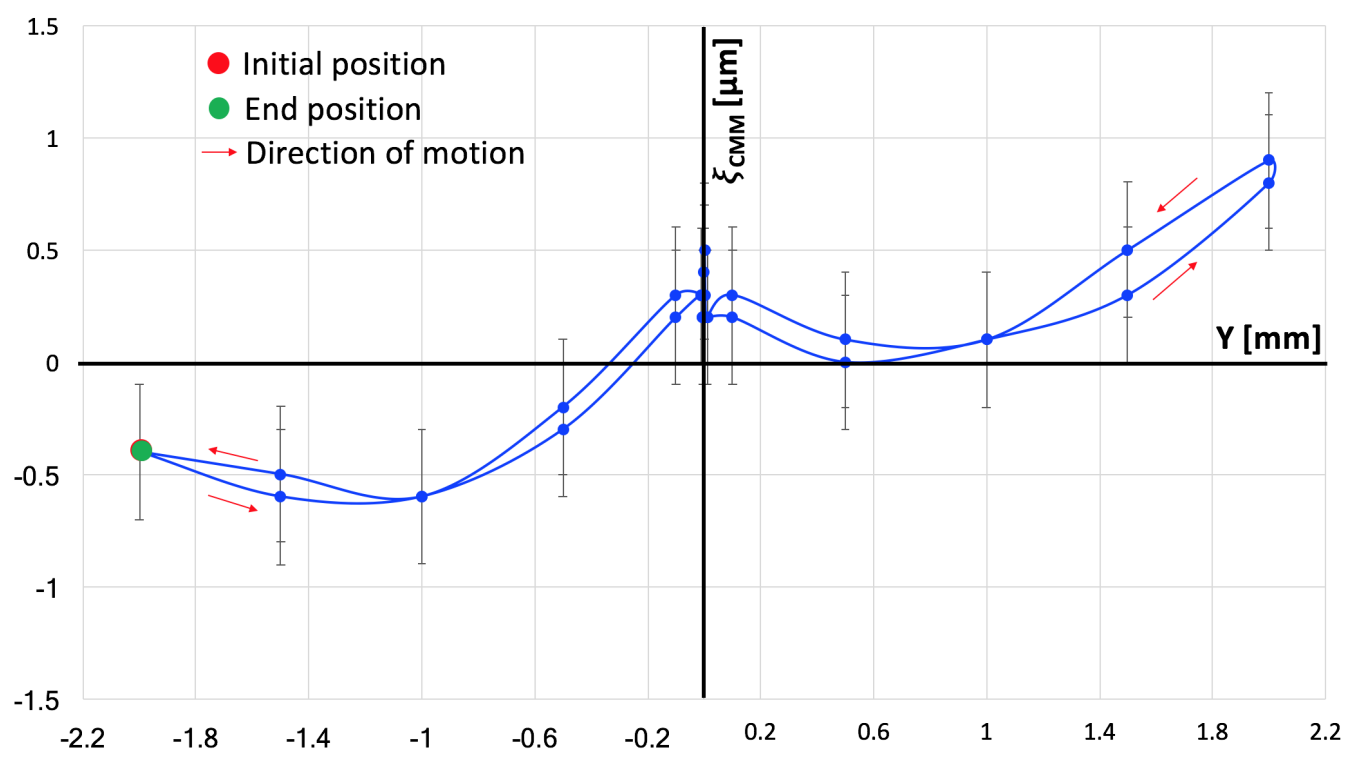

(a)

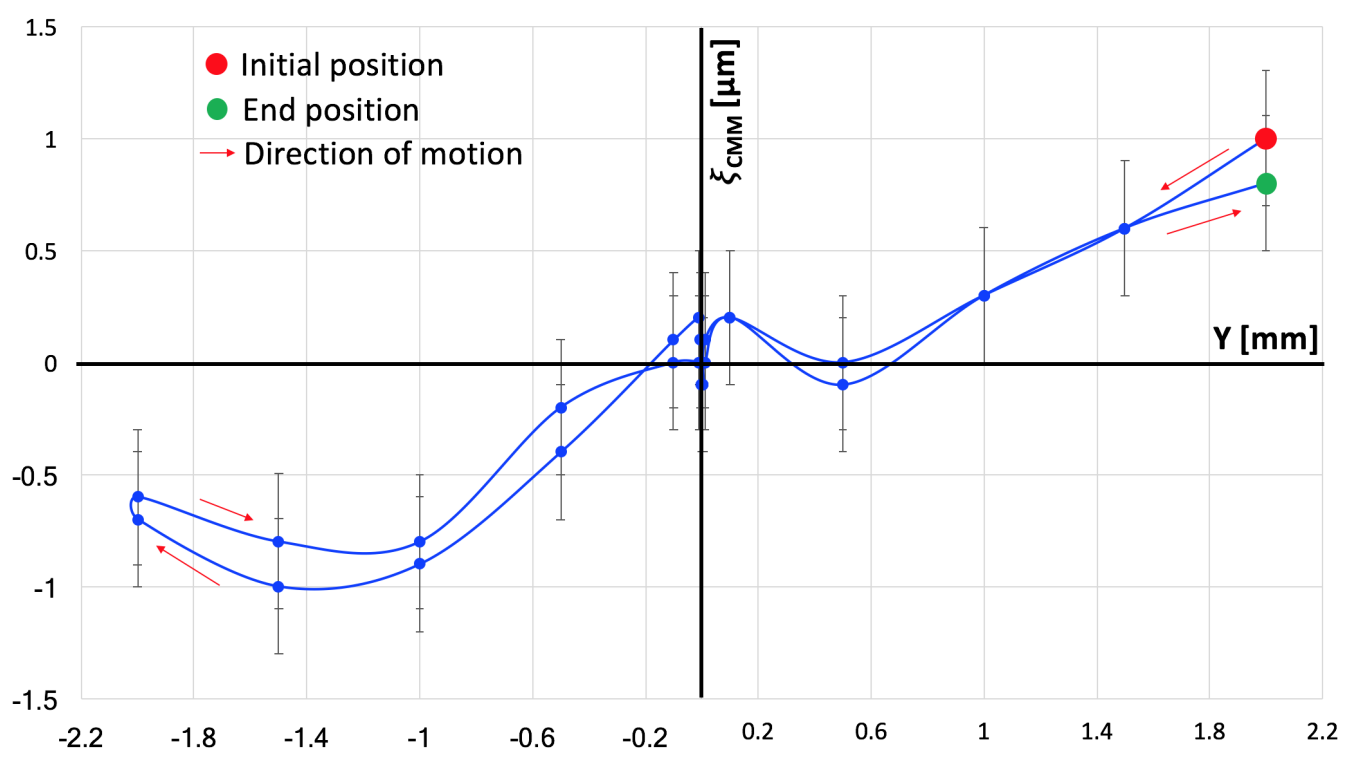

(b)

Figure 3.15: Measurement of the accuracy of $Y$ with a load of $25 \mathrm{~kg}$ when both linear stages are moved at the same time in (a) Test 3 and (b) Test 4.

\subsubsection{Calibration of the wire tilt}

One of the most important calibrations performed in the metrology laboratory at CERN was the perpendicular wire positioning with respect to the base where the AS stands vertically, with a controlled room temperature of $20 \pm 1^{\circ}$. This adjustment allows the minimisation of the wire tilt inside the AS. For this measurement, the accelerating structure was not required and the wire was installed and stretched at both extremes of the frame, as shown in Figure 3.16. The measuring instrument used for this purpose was the CMM 


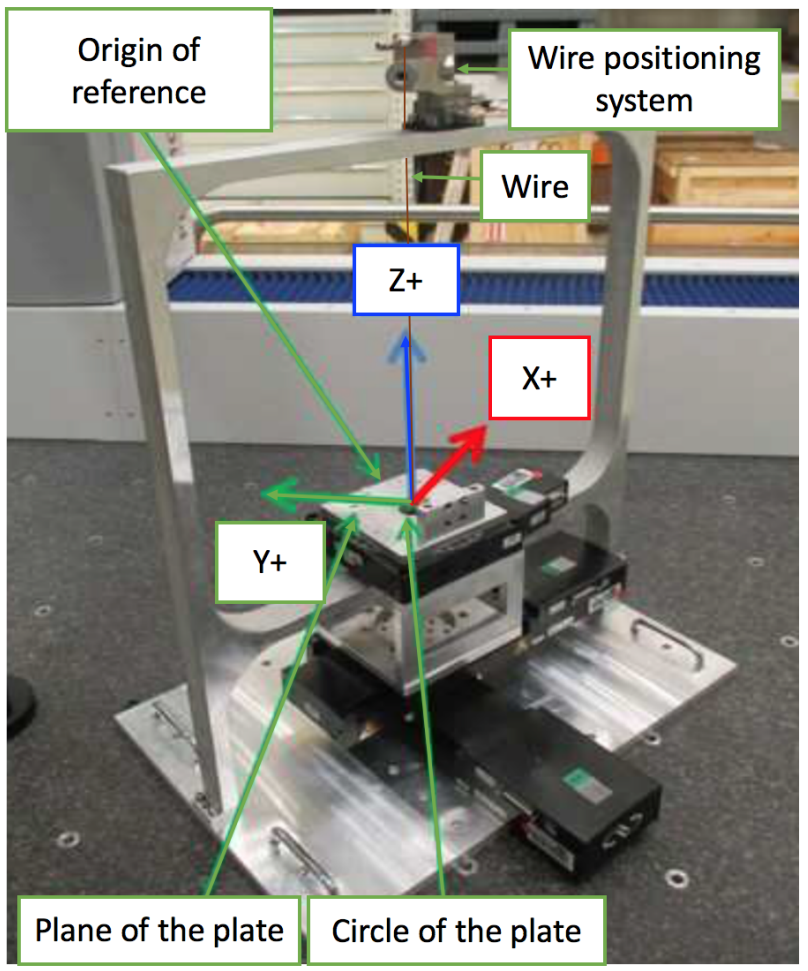

Figure 3.16: Wire calibration in the ZEISS PRISMO CMM.

model ZEISS PRISMO with an uncertainty dependence with the length of the device under test, $L$, following:

$$
E_{0}[\mu m]= \pm 1.2[\mu m]+L[m m] / 500
$$

A reference system was defined at the aluminium base. To form any reference system, a reference point and a plane are needed. The reference point defined as the origin of reference, see Figure 3.16, was found by measuring some points around the circle in the middle of the plate with the CMM, whose centre was calculated by the CMM software. This point, together with the plane of the plate, formed the reference system.

Later, the wire was installed and stretched. The three fiducials equipped in the wire positioning system on top of the frame, detailed in Figure 3.5, are disposed in an equilateral triangle whose centre gives the wire position. The spheres were measured with the same CMM and the software delivered the wire position. This way, the angle of the straight line perpendicular to the aluminium plate with respect to the wire position was known.

After these measurements, the correction on the positioning was done using the two orthogonal micrometers where the top wire positioning system stands, Figure 3.16. This process was iterated several times until the achievable wire tilt was satisfactory enough: the resulting wire was included in a cylinder with a length of $341.1 \mathrm{~mm}$ and a radius of $11 \mu \mathrm{m}$, corresponding to a tilt in the middle cell of $0.0025^{\circ}$. 
Chapter 3: Design, fabrication and calibration of an experimental set-up to measure the EM axes of the CLIC TD24 using the perturbative method

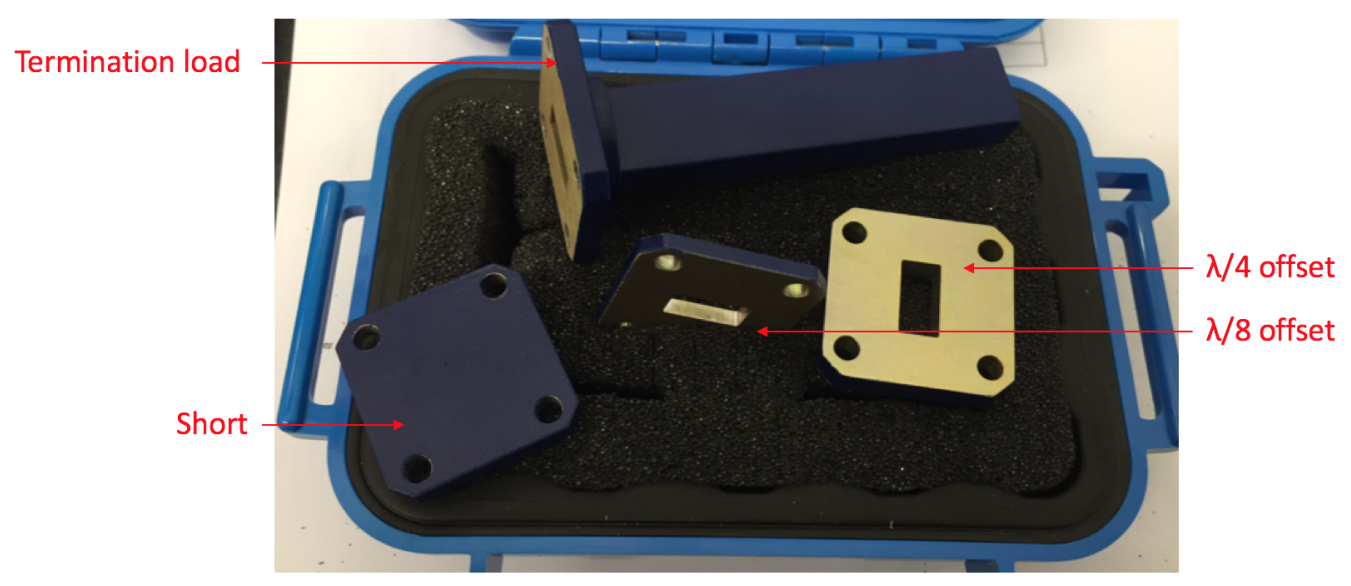

Figure 3.17: Calibration kit of standard waveguide WR51.

\subsubsection{Calibration of the VNA}

A manual four-port VNA calibration is required before the EM measurement process to avoid drift errors caused by variations at VNA performance arising from variations in ambient temperature. The systematic errors due to VNA, cables and transitions imperfections are minimised with a calibration data properly loaded in the VNA and a measurement calibration completed.

The calibration is done at the plane of the transition SMA-WR51 using a calibration kit for standard waveguide WR51, see Figure 3.17. The calibration data should be properly loaded in the VNA in order to complete the measurement calibration. For this purpose, the calibration definitions for the devices in the kit must be installed prior to performing a calibration, following carefully the instructions displayed in the VNA when selecting the option Add new calibration kit. The kit contains a termination load, an offsets of $1 / 4$ wavelenght $(\lambda / 4)$, an offset of $1 / 8$ wavelength $(\lambda / 8)$ and a short. From the two available offset standards, $\lambda / 4$ was chosen. The offset delay is given by:

$$
\text { Offset delay }[n s]=\frac{l[\mathrm{~mm}]}{299.6953 \frac{[\mathrm{mm}]}{[n s]}}
$$

Where $l$ is the section of the $\lambda / 4$ element, and the denominator is the value of the wave propagation velocity in air. The resulting offset delay is $18.35 \mathrm{ps}$, which corresponds to a phase shift of $76.4^{\circ}$.

The routine VNA calibration involves the setting of the following parameters: start and stop frequencies, the number of points required for a frequency sweep and the measurement bandwidth. After that, the calibration kit that has just been defined in the instrument can be selected, and the manual four-port calibration can be performed. Once finished, each port can be connected to the middle cell of the AS, and the measurement process of the EM centre can start. 


\subsection{Automatisation of the measurements}

\subsubsection{Conceptual design of the algorithm}

The algorithm development and its optimisation is one of the most critical parts of this work. The development process is directly linked to the theoretical concepts explained in detail in Chapter 2 and the results obtained via computer simulation using HFSS. The optimisation factor comes exclusively with the experience and the knowledge acquired during the measurements in the laboratory, that will be presented in Chapter 4 . The final algorithm is shown schematically in Figure 3.18 and consists of:

\section{Calibration of the VNA:}

The starting point of a measurement is the calibration of the instrument equipment that measures the $S$ parameters: the VNA.

\section{Connect the VNA ports to the AS:}

Once the calibration process has been completed, we connect the four ports of the VNA to the taper transitions that are assembled in the middle cell of the AS, Figure 3.1. Note that the connections should follow the port definitions as shown in Figure 4.11. Therefore, the port numbers are localised following a clockwise direction: $\mathrm{p} 1$ is defined in the negative direction of the linear stage in the $X$ plane, $\mathrm{p} 2$ is fixed to the positive direction in $Y$, and so on.

\section{Installation of the wire:}

The wire is passed through the interior of the AS, it is stretched and both extremes are grabbed using the wire-positioning systems (Figure 3.5).

4. Set parameters in the user interface:

The parameters are set in the main user interface in LabVIEW, and the automatic measurement process can start without the presence of the user.

5. Move $X, Y$ and $\varphi$ to home position:

Both linear and rotation stages are driven to home position following:

$$
\begin{array}{r}
X=0.0000 \pm 0.0001[\mathrm{~mm}] \\
Y=0.0000 \pm 0.0001[\mathrm{~mm}] \\
\varphi=0.0000 \pm 0.0001 \text { [degrees] }
\end{array}
$$

\section{Fix $Y, \varphi$ to home position. Move the AS in $X$ :}

The linear stage in the $Y$ axis and the rotation stage remains fixed to the home position, while the linear stage in the $X$ axis moves along the selected travel range and step size in the LabVIEW settings. As already mentioned, ports are physically defined and localised as shown in Figure 4.11 (a). 


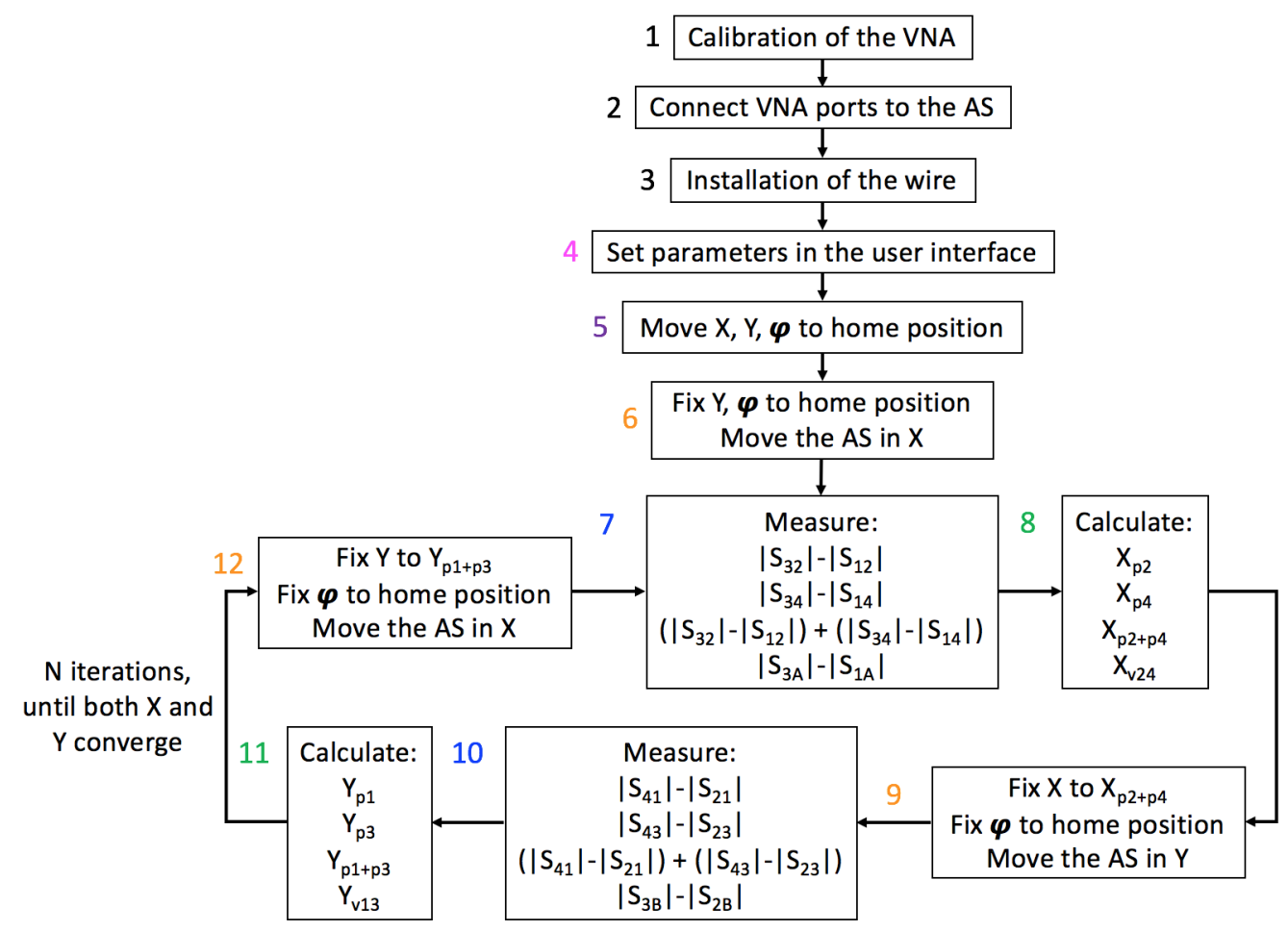

Figure 3.18: Algorithm for the experimental measurements.

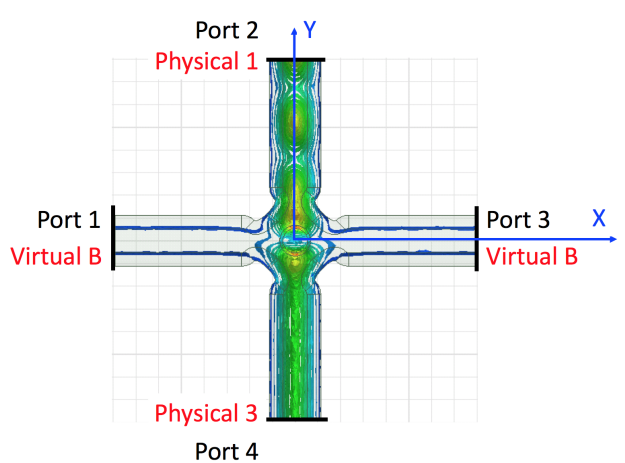

(a)

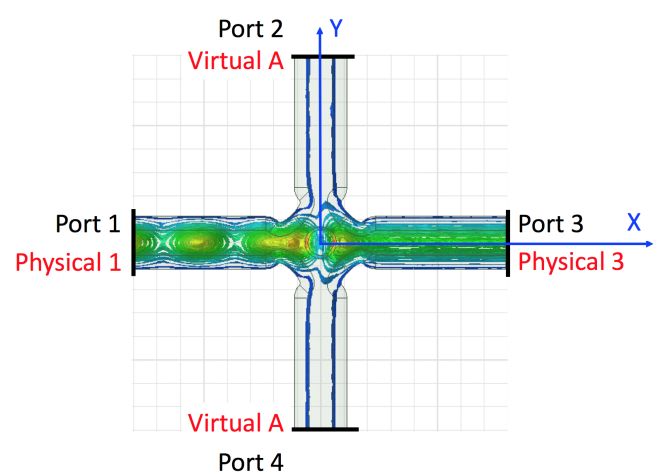

(b)

Figure 3.19: (a) Port connections in the middle disk of the CLIC TD24 accelerating structure when the linear stage in $Y$ remains fixed to a position and the linear stage in the $X$ moves (step 7 in the algorithm). (b) Port connections in the middle disk of the CLIC TD24 accelerating structure when the linear stage in the $X$ remains fixed to a position and the linear stage in $Y$ moves (step10 in the algorithm). 


\section{Measure:}

A full 4-port $S$-parameter matrix is measured at each position. As justified with EM field simulations and with experimental measurements in Chapters 2 and 4, respectively, the following relationships are calculated:

$$
\begin{gathered}
\left|S_{32}\right|-\left|S_{12}\right| \\
\left|S_{34}\right|-\left|S_{14}\right| \\
\left(\left|S_{32}\right|-\left|S_{12}\right|\right)+\left(\left|S_{34}\right|-\left|S_{14}\right|\right)
\end{gathered}
$$

Later, the physical ports 2 and 4 are defined as a common virtual p2 -renamed A-, see Figure 4.11 (a). At each position, the relationship between the ports is measured as:

$$
\left|S_{3 A}\right|-\left|S_{1 A}\right|
$$

\section{Calculate:}

Following the explanations in Chapter 2, the four measurements are linearly fitted, and their crossing with zero deliver four points whose values are the positions where the perturbation is minimised as:

$$
\begin{gathered}
\left|S_{32}\right|-\left|S_{12}\right|=0, \text { at } X=X_{p 2} \\
\left|S_{34}\right|-\left|S_{14}\right|=0, \text { at } X=X_{p 4} \\
\left(\left|S_{32}\right|-\left|S_{12}\right|\right)+\left(\left|S_{34}\right|-\left|S_{14}\right|\right)=0, \text { at } X=X_{p 2+p 4} \\
\left|S_{3 A}\right|-\left|S_{1 A}\right|=0, \text { at } X=X_{v 24}
\end{gathered}
$$

\section{Fix $X$ to $X_{p 2+p 4}$. Fix $\varphi$ to home position. Move the AS in $Y$ :}

Later, the linear stage in the $X$ is brought to the position $X_{p 2+p 4}$, while the rotation stage remains always to home position. The $Y$ axis moves with the travel range and step size indicated in the main user interface in LabVIEW. In this part of the algorithm, the ports are designated as in Figure 4.11 (b).

10. Measure:

At each position, the $S$ parameters are measured and the following relationships are calculated as:

$$
\begin{gathered}
\left|S_{41}\right|-\left|S_{21}\right| \\
\left|S_{43}\right|-\left|S_{23}\right| \\
\left(\left|S_{41}\right|-\left|S_{21}\right|\right)+\left(\left|S_{43}\right|-\left|S_{23}\right|\right)
\end{gathered}
$$

After these three measurements, ports 1 and 3 are defined as common virtual p1 -renamed B-, physical p2 remains the same and physical p4 is named logical p3, see Figure 4.11 (b). This way, the following measurement is performed as:

$$
\left|S_{3 B}\right|-\left|S_{2 B}\right|
$$


Chapter 3: Design, fabrication and calibration of an experimental set-up to measure the

\section{Calculate:}

The points found from each measurements are linearly fitted. Their crossing at zero give the positions where the linear stage in $Y$ passes through the position where the wire causes a minimum perturbation of the EM field and the following points are obtained:

$$
\begin{gathered}
\left|S_{41}\right|-\left|S_{21}\right|=0, \text { at } X=X_{p 1}->\text { Renamed } Y_{p 1} \\
\left|S_{43}\right|-\left|S_{23}\right|, \text { at } X=X_{p 3}->\text { Renamed } Y_{p 3} \\
\left(\left|S_{41}\right|-\left|S_{21}\right|\right)+\left(\left|S_{43}\right|-\left|S_{23}\right|\right)=0, \text { at } X=X_{p 1+p 3}->\text { Renamed } Y_{p 1+p 3} \\
\left|S_{32}\right|-\left|S_{12}\right|=0, \text { at } X=X_{v 13}->\text { Renamed } Y_{v 13}
\end{gathered}
$$

12. Fix $Y$ to $Y_{p 1+p 3}$. Fix $\varphi$ to home position. Move the AS in $X$ :

$Y_{p 1+p 3}$ is chosen for the next iteration as a fixed position in $Y$. The rotation stage $\varphi$ remains at home and $X$ moves again.

Steps from 7 to 12 are repeated $\mathbf{N}$ times until the results of the EM centre converge in both axes. In the first iteration of the algorithm, the measurements are done along the diameter of the structure which is around $4 \mathrm{~mm}$. In the next iterations, the step size is reduced to the minimum resolution value with a travel range on the stages big enough to perform the linear fit with enough number of points. These results are explained in Chapter 4. The code colour in Figure 3.18 corresponds with the LabVIEW Figures from 3.21 to 3.23 .

\subsubsection{Software development for the automated algorithm performance}

The algorithm is monitored from steps 5 to 12, with the implementation of a user platorm programmed in LabVIEW that has been developed within this project. The loop of the algorithm allows the programming of functions that are recalled, repetitively, along the execution of the program. The functions are programmed as LabVIEW Virtual Instruments (VI) and will be described in this subsection. Despite that, this code can still be optimised, this version is fast - it takes around 12 seconds per measured point -, simple and good enough to perform the measurement of the EM centre in the AS.

Two iterations, $\mathrm{N}=2$, are implemented in the main user platform with the possibility of setting different travel ranges of the linear stages and step sizes, depending on the requirements of the user. The user interface is shown in Figure 3.20 (a) and the following input parameters are observed: GPIB address of the VNA, GPIB address of the controller ESP300, starting positions of the stages $(X, Y, \varphi)$, travel range and step size. The frequency where the EM centre is measured can be configured directly in the block diagram code. The user must set the listed parameters in the front panel when performing step 4 of the algorithm.

The block diagram of the main program is presented in Figure 3.22. On the left side of the code, all the input variables are laid out. After the initialization of the variables, a sequence structure with five frames is implemented in order to assure the correct sequencing of the algorithm. The sequence is also guaranteed with the input-output error 
variable. The error variable allows the execution of a function once the previous code has been executed successfully. On the first frame in Figure 3.22, the stages are programmed to execute steps from 5 to 8 . The programming is done independently in a subVI named Xhome.VI. In the next frame, another subVI where $\mathrm{Y}$ is programmed to be moved while $\mathrm{X}$ stays fix is called under the name Y.VI, executing from steps 9 to 11. Later, the algorithm loop is repeated again by executing steps 12, 7 and 8 in X.VI and executing again steps from 9 to 11 in Y.VI, corresponding to the third and fouth frames, respectively in Figure 3.22. Finally, in the last frame, the output parameters of each subVI are stored in a file. Since the next iterations do not require the initialisation of the stages at home, a variant of the main.VI has been programmed. This code simply replaces the Xhome.VI by the X.VI and the rest remains the same.

The subVI called in the first frame of the main code is now described, named as Steps: 5, 6, 7, 8 in Figure 3.22. The same explanations can be applied to the subsequent subVI executed in the following frames. The input parameters required for its execution are shown in the Front Panel in Figure 3.20 (b), and the output variables of the function are shown in Figure 3.20 (c). The execution of this function returns the plot of the four measured relationships at (3.4), (3.5), (3.6) and (3.7) with respect to the position of the linear stage that is moving. The solutions given by (3.8), (3.9), (3.10) and (3.11) are also returned. The code that allows all this is shown in the block diagram displayed in Figure 3.23.

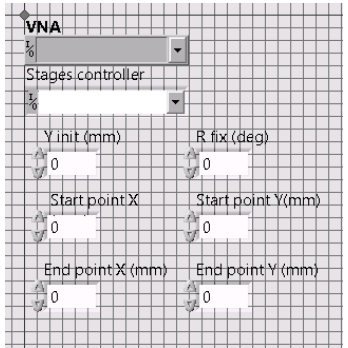

(a)

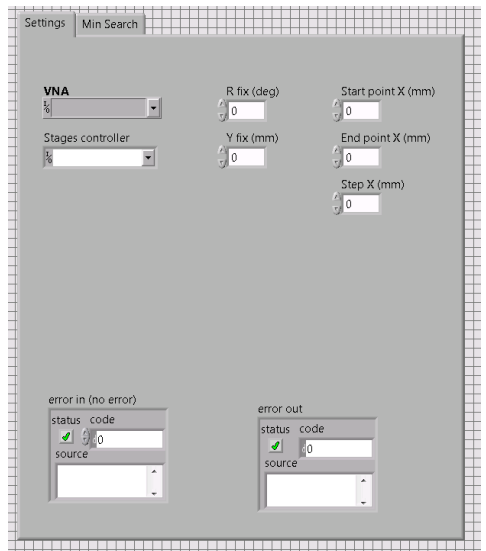

(b)

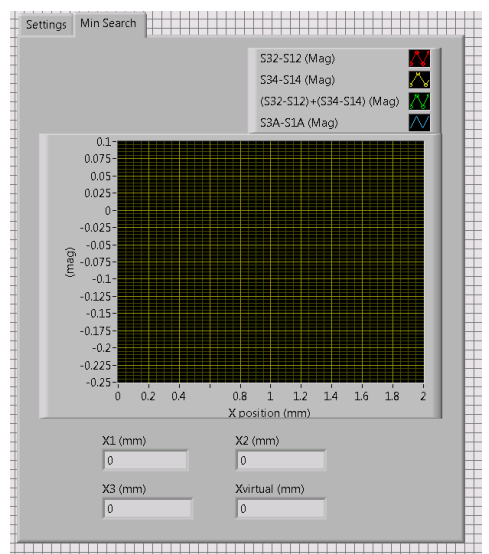

(c)

Figure 3.20: (a) Main user interface in LabVIEW with setting parameters to measure the EM centre of AS, performing steps from 5 to 12. (b) Settings of the VI that performs steps from 5 to 9. (c) Parameters returned at the end of step 9.

The control of the ESP300 has been done by slightly modifying an available code at the National Instruments' website. They correspond in Figure 3.23 to the boxes named ESP. As signalised in the pink rectangle, they are called to switch on the stages. Later, in the violet part, the stages are set to their home positions, step 5 of the algorithm. This block is only done once and is the only part that is not repeated in X.VI and Y.VI in the next iterations. After that, $\mathrm{Y}$ is fixed to a position and a for loop starts -orange rectangle or step 6 of the algorithm-. The starting and stop points of the loop are the first and last position of the linear stage in $X$. The loop iterates proportional to the number of steps. The 
Chapter 3: Design, fabrication and calibration of an experimental set-up to measure the

position of the linear stage in $X$ is settled at every iteration of the loop (orange rectangle inside the loop in Figure 3.23), and the $S$ parameters are measured (blue rectangle inside the loop in Figure 3.23).

The control of the VNA is done using the LabVIEW attribute-based drivers provided by Rhode\&Schwarz to simplify the development process of instrument remote control applications. These drivers utilise the Express VI technology, which internally takes advantage of VI scripting about programmatically creating and modifying VIs. Two modifications were applied with input parameters: frequency start, frequency stop, number of points, input port and output port. The function returns a file with the measured $S$ parameters that have been indicated as input and output ports. The data is stored as real and imaginary values of the $S$ parameter at the indicated frequency range. The details about these two functions are explained later.

Once all $S$ parameters have been measured, LabVIEW performs the required operations in order to combine the $S$ parameters of interest. The green block highlighted in green in Figure 3.23, represents calculation of the EM centre at the chosen frequency in $X$. At this point, the main VI uses the calculated $X_{p 2+p 4}$ as input parameter in the next subVI. This time, the same process is repeated, and the EM centre in $Y$ is returned and given to the next sub.VI as input parameter.

As mentioned, two variants of VNA coding were required, see Figure 3.21, where the original code provided by Rhode\&Schwarz was modified. The subVI in Figure 3.21 (a) allow the measurement of the $S$ parameters through four physical ports, while the other subVI in Figure 3.21 (b) combine two physical ports in one logical port. This way, the instrument has one common stimuli input port and one single-ended output port, to operate virtual-port measurements. This code accepts: the input and output ports of the VNA, the GPIB address of the VNA, the name of the file to record the data and the start and stop frequencies for the sweep. Note that the number of points and other configurable parameters of the VNA like power, can also be configurable by entering them directly in the VNA.VI code. 


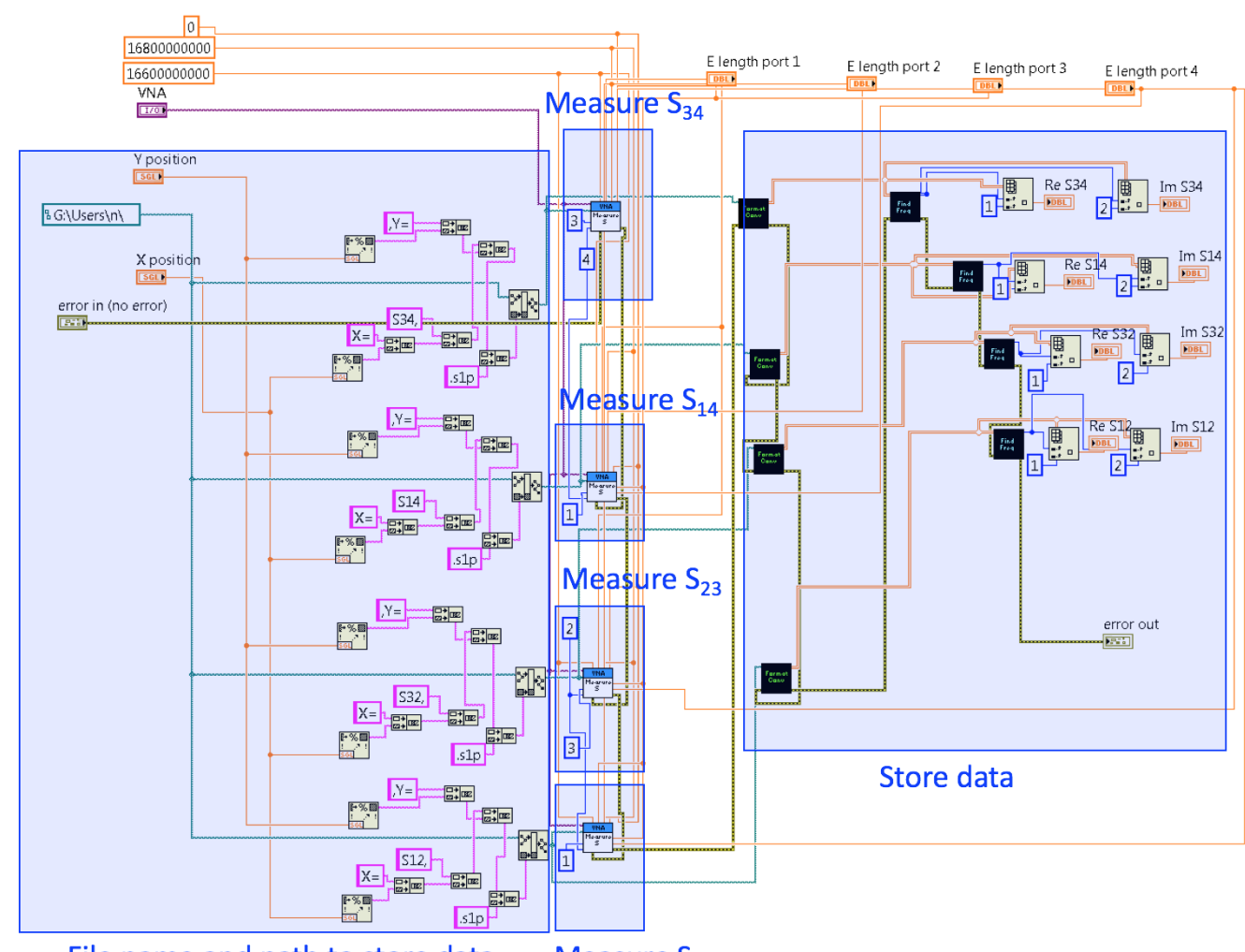

File name and path to store data $\quad$ Measure $\mathrm{S}_{13}$

(a)

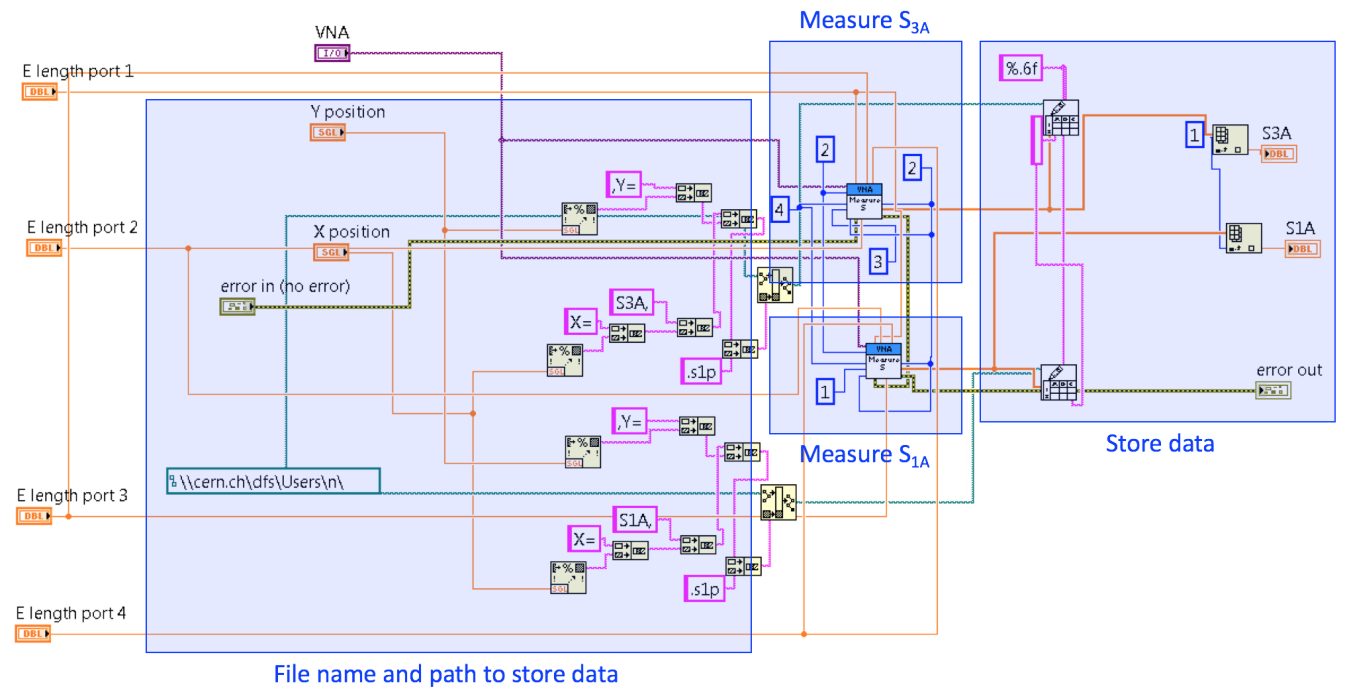

(b)

Figure 3.21: Block diagram to perform the measurement of the $S$ parameters (a) using the physical ports of the VNA corresponding to the first part of step 7 in the algorithm diagram (Figure 3.18) and (b) using the virtual ports of the VNA corresponding to the second part of step 7 . 


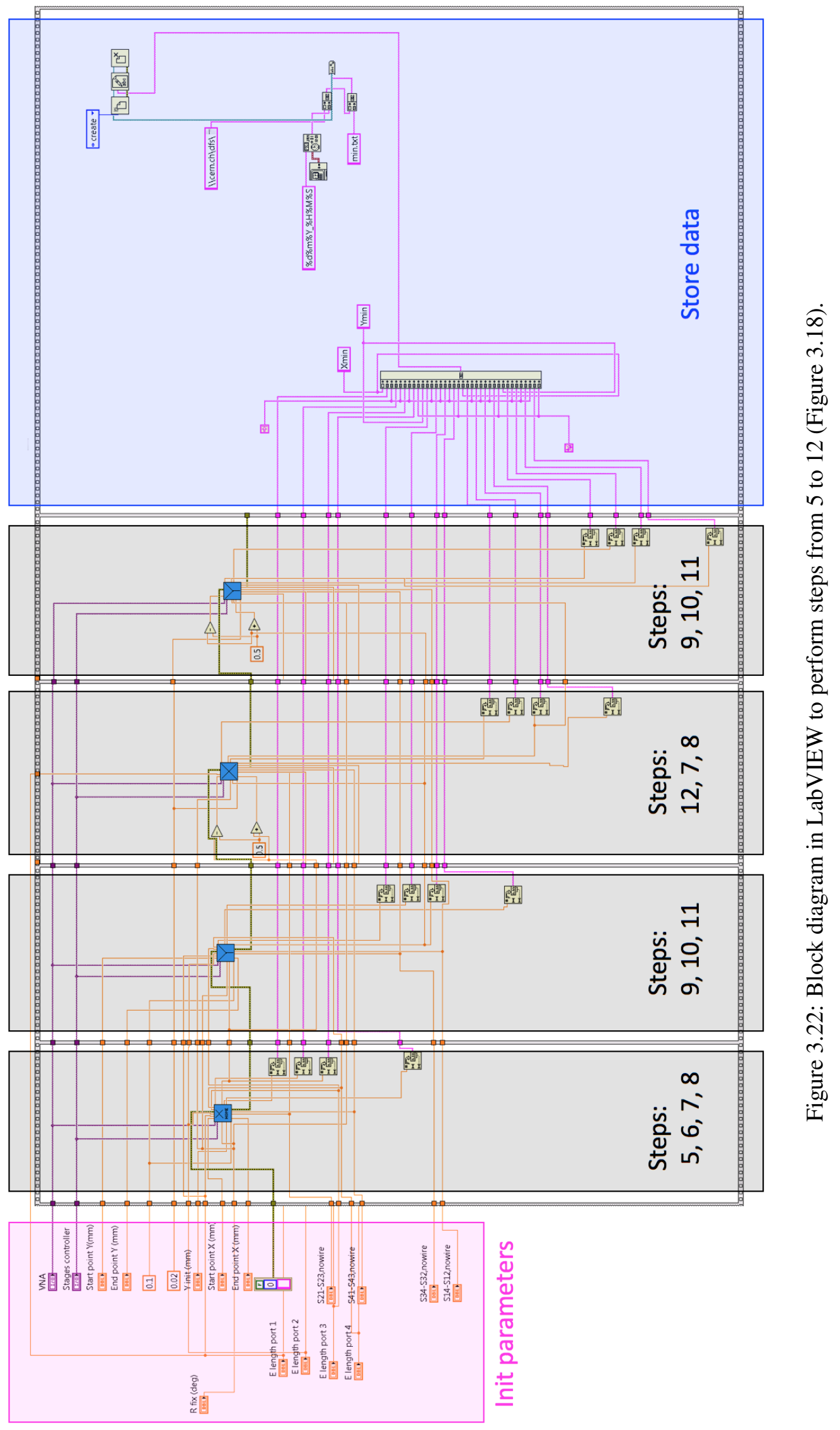




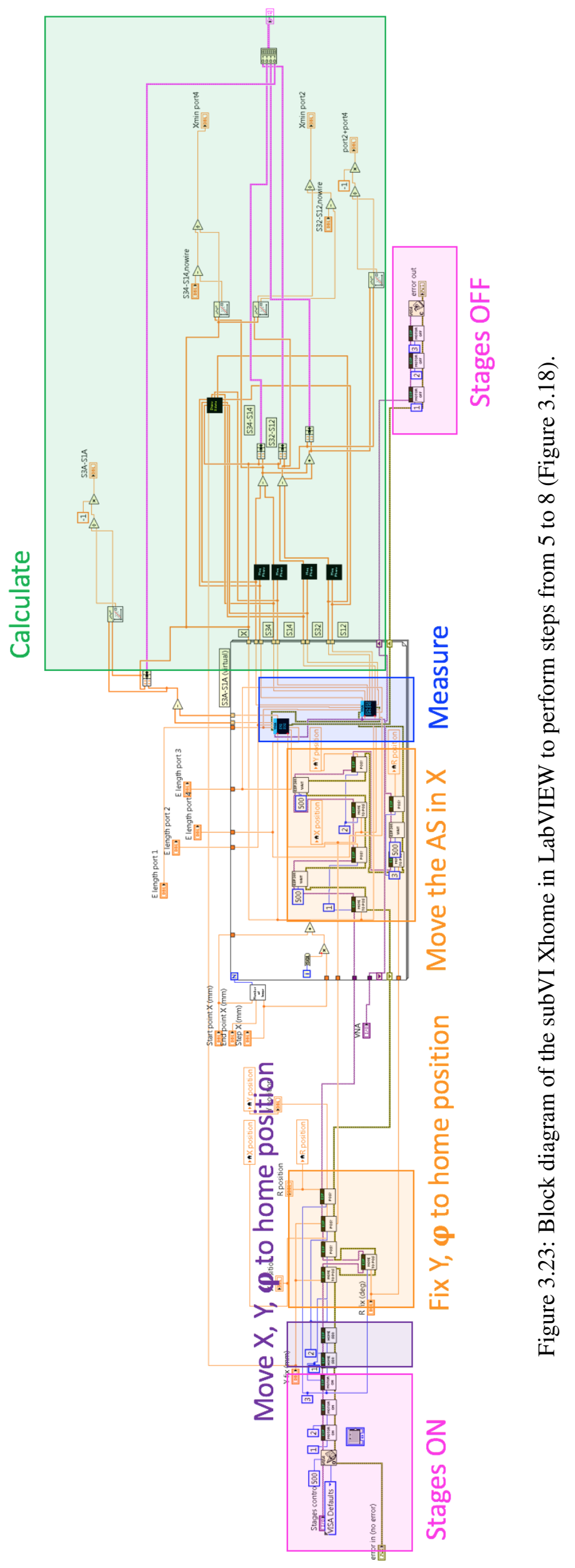




\subsection{Further developments of the measurement of the EM cen- tre of AS}

All the measurements described can be applied to all cells using the same method. Two lines of investigation were performed for three months at NI's Open Laboratory in Debrecen, Hungary, as part of the PACMAN training. We established a close collaboration with the NI RF engineers to explore the feasibility of innovative applications of the method using the latest NI technology. In the next subsections both ideas are explained: one to apply the method to measure the EM centre in all cells of the AS, while in the other one the idea is to locate the EM centre in the first, middle and last cells of the AS, labeled in Figure 3.1.

In the last subsection, the design of a second test bench allowing the measurement of the EM axes in the TD24 in the direction of the beam is described.

\subsubsection{The cell-to-cell misalignment measurement}

The interest of measuring the EM centre in all cells is to compare the EM results with external metrology measurements on the disks of the structure, and evaluate the possible correlation between them. If the RF measurements turn out to be more accurate than metrology, the cell-to-cell measurement may infer in the sequence of the fabrication process. If it is eventually performed before bonding, the misalignment tolerances between disks can be assured. The process would be the following one.

All disks, at exception of the middle one, are equipped with pumping holes at the end of the waveguides with a diameter of $4.2 \mathrm{~mm}$, see Figure 3.1, that could be used as input and output ports. Special feedthroughs have been purchased from two different providers. They are SMA connectors that can be directly connected to the SMA cables of the VNA. The first connector considered is the model 60-02-9E2-093B [95] from the company CMPTER. The connector can be seen in Figure 3.24 (a) and it consists on a stainless steel body and a copper beryllium pin contact. The cylindrical contact has $3.8 \mathrm{~mm}$ of diameter, which makes possible the connection through the pumping holes in the accelerating structure. The second connector purchased was the model R125414000W from Radiall -see Figure 3.24 (b)- with a working frequency up to $24.5 \mathrm{GHz}$. The contact material is copper beryllium and the impedance is $50 \Omega$. This connector allows the connection through the pumping holes of the structure thanks to its $4.1 \mathrm{~mm}$ of diameter cylindrical contact and the $12.7 \mathrm{~mm}$ square flange jack receptacle, perfect for a coaxial transmission line [96]. Two conceptual designs to be performed are: cable clamps to grab the heavy VNA cables, and perfect electrical connections between the pumping holes and the feedthroughs. 


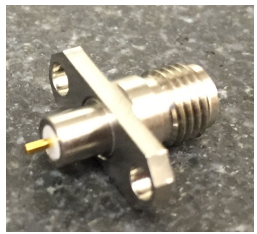

(a)

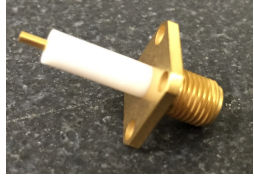

(b)

Figure 3.24: Feedthrough connectors considered for a cell-to-cell misalignment measurement. On the left, SMA from CMPTER and on the right, SMA from Radiall.

\subsubsection{The measurement of the EM axes simultaneously in various cells}

Following the same idea about measuring the EM centre in all disks of the TD24 accelerating structures, a solution was investigated in order to measure the EM centre in three cells simultaneously. This performance would allow the measurement of the actual wire tilt inside the AS, and its correction using the micrometers on top of the frame at the same time as a measurement of the average EM centre in the whole AS similar to the EM centre that the beam experiences when it is steered through the AS. The aim is to connect the first, the middle and the last disk directly to a VNA. The connection between the VNA and the cells would be done using the feedthroughs presented previously. This development requires the motorisation of both micrometers in order to push down the time consuming of the measurements, and provide a reliable positioning.

The solution proposed in order to connect the AS cells to the VNA, is based on the PCI eXtensions for Instrumentation (PXI) technology developed by NI. It consists of a switching network with an insertion loss of $0.3 \mathrm{~dB}$ and $26.5 \mathrm{GHz}$ performance. The schematic of the set-up would be as follows: the accelerating structure would be mounted on the movers, whose controller is wired in series configuration through the GPIB port to the NI controller, the VNA and the NI chassis. The four ports of the middle cell, together with the four ports of the first and last cell, would be connected to the switching network. Finally, a LabVIEW user interface would be programmed in the controller of the chassis. The feasibility of the solution depends on the resolution of the readable value of the whole system. This was tested with characterisation of a $1.4 \mathrm{GHz}$ to $2.8 \mathrm{GHz}$ hybrid directional coupler in the laboratory, and with the extension of a two-port VNA to four ports.

The equipment wiring is shown in Figure 3.25. The NI chassis NI PXIe-1085 pictured in Figure 3.26 was chosen to host the NI-PXI boxes forming the network. An embedded Controller - model NI PXIe-8135- was installed in the first slot in order to drive the movers, the VNA and the switching network by the LabVIEW interface. A two-port VNA with a frequency range from $300 \mathrm{kHz}$ to $8.5 \mathrm{GHz}$ built with PXI technology was installed in the chassis. The model is the NI PXIe-5632. And finally, the switching network was formed by three dual SPDT relays and two dual 6x1 multiplexer ST6T. The dual SPDT relays chosen are the model NI PXI-2599, with $0.3 \mathrm{~dB}$ insertion losses at a working frequency of $26.5 \mathrm{GHz}$. And the model of the $6 \times 1$ multiplexer was the NI PXI-2596 with $0.3 \mathrm{~dB}$ insertion losses at $26.5 \mathrm{GHz}$. 
One of the major complexities of this measurement concerns the calibration process. For this reason, an existing LabVIEW user interface to calibrate the VNA NI PXIe-5632 with an electronic calibration kit, was modified for our purposes. The resulting code allowed the calibration at the end of the cables in the switching network, with the selection of the different routes when connecting the ports two by two. The front panel is shown in Figure 3.27 (a). The $S$ parameter measurements were done using an existing interface developed by NI, that was modified in order to fulfil our purposes, resulting the front panel in Figure 3.27 (b).

Three measurement tests were performed in order to estimate the resolution of the system. The first test consisted on an attenuation measurement of the switching network. The transmission between the two ports of the VNA were measured with and without switches, and calibration was not required here. On the second test, the switching equipment was not used and a calibration process was performed. The test consisted on an output signal from one of the ports of the VNA that excited the different ports at the directional coupler, one at a time, while the rest remained loaded at 50-ohm. The third test consisted in performing the same measurement as the second test using the switching network. The measurements results obtained coincided with those specified in the technical datasheet: bandwidth, isolation and $S$ parameters. In addition, the measured $S$ parameters at tests 2 and 3 were the same ones, meaning that the switching network can be calibrated and that a 4-port extension of the VNA ports is possible without losing precision on the measurements. The measurements were done in a bandwidth from $500 \mathrm{MHz}$ to $4 \mathrm{GHz}$, so the calculations about insertion loss introduced by each switch should be done for the range of frequencies where the measurements through the accelerating structure is done. The insertion loss introduced by each switch in the tested bandwidth was $0.1 \mathrm{~dB}$. At high frequencies, the value specified in the datasheet was around $0.5 \mathrm{~dB}$ maximum. Additional attention should be payed to the little reflections caused by the coupling between the switching ports even when terminated at $50 \Omega$, which is expected to be higher at higher frequencies. Thus, the proposed equipment for the 12-port VNA solution looks like in Figure 3.28. It involves the following elements:

- Chassis model NI PXIe-1085 with 18 slots.

- The embedded controller PXI-8840 for PXI systems.

- Two dual 6x1 multiplexers with a working frequency up to $26 \mathrm{GHz}$, model NI PXI2596.

- Six dual SPDT switches up to $26 \mathrm{GHz}$, model NI PXI-2599.

- Eight SMA male to SMA male flexible cables with a lenght of $38.1 \mathrm{~cm}$.

The procedure to calibrate the twelve ports is the same one as explained before. In the picture, the input power from the VNA is represented with brown wires and the output power with blue wires. All the paths would be defined and calibrated in LabVIEW by adapting the previous calibration software to the ZVA24 VNA. The calibration files would be used in the main user platform developed in LabVIEW for this application with the corresponding defined paths. 


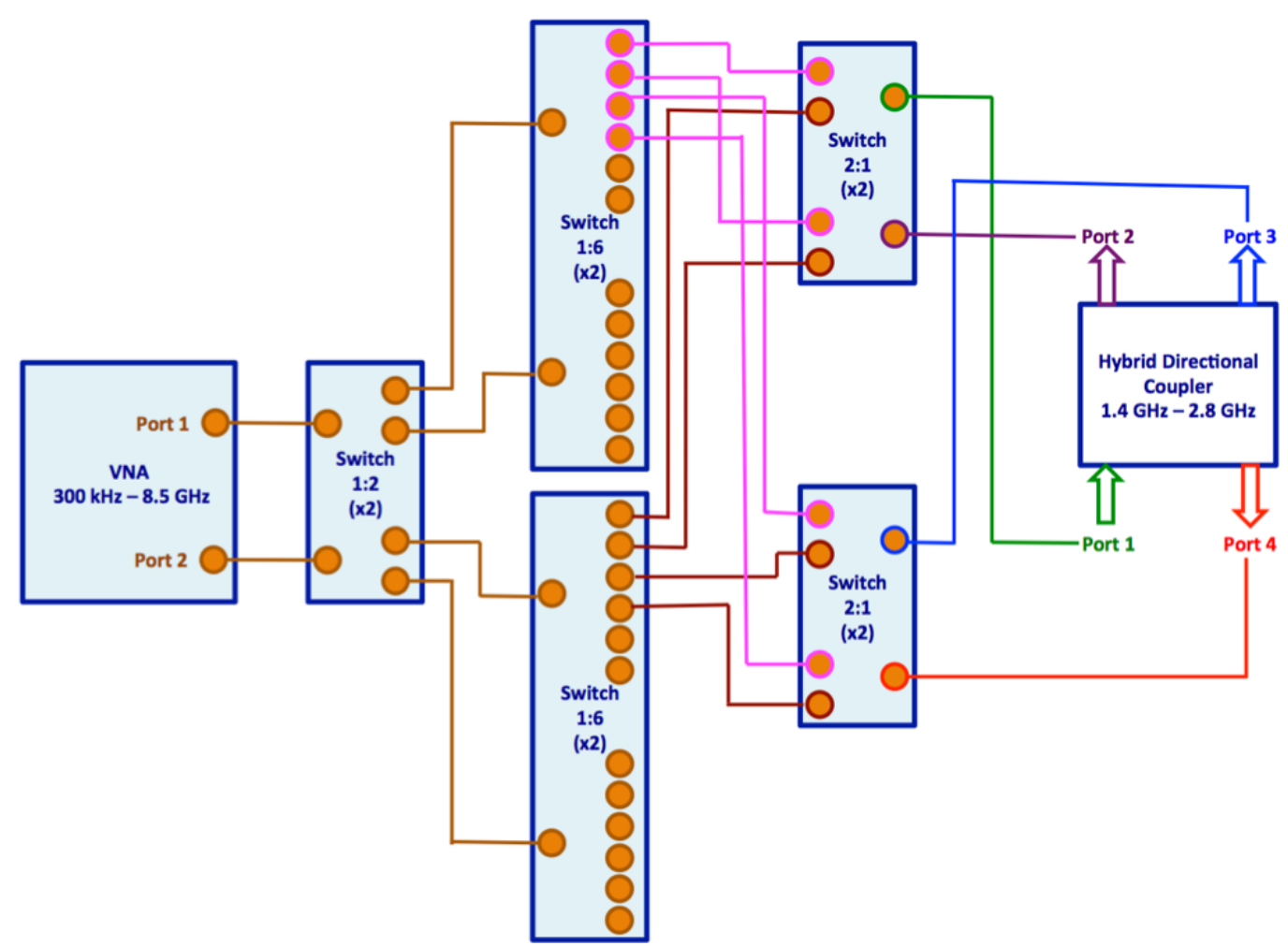

Figure 3.25: Set-up for the characterisation of a hybrid directional coupler with a switching network with NI technology to extend a two-port VNA to four ports.

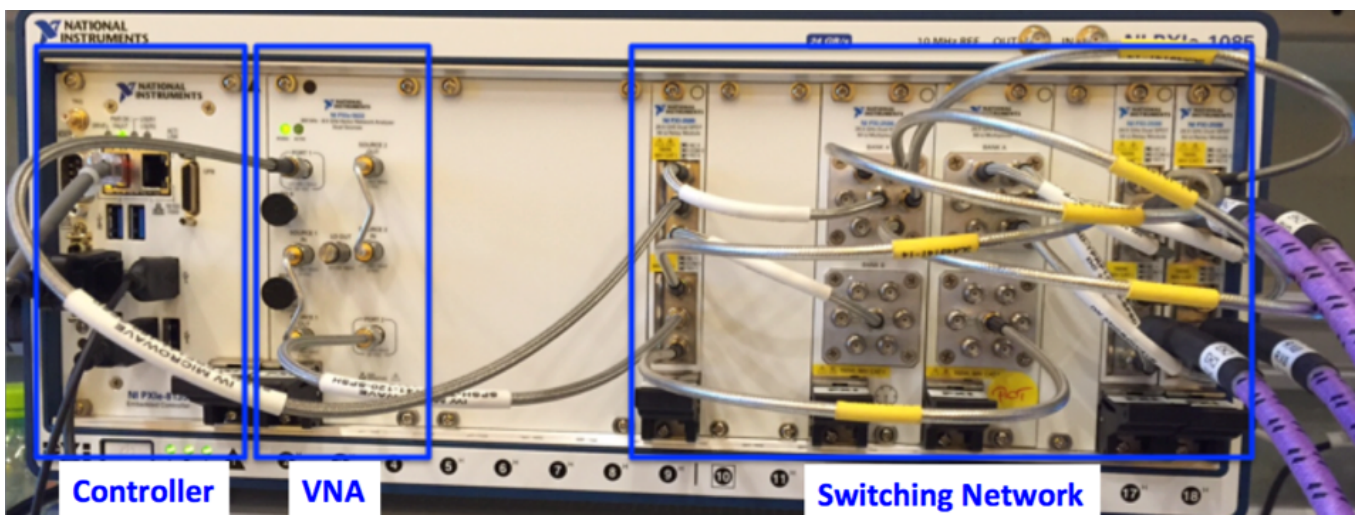

Figure 3.26: NI chassis with the instrument equipment made of PXI technology. 
Chapter 3: Design, fabrication and calibration of an experimental set-up to measure the EM axes of the CLIC TD24 using the perturbative method

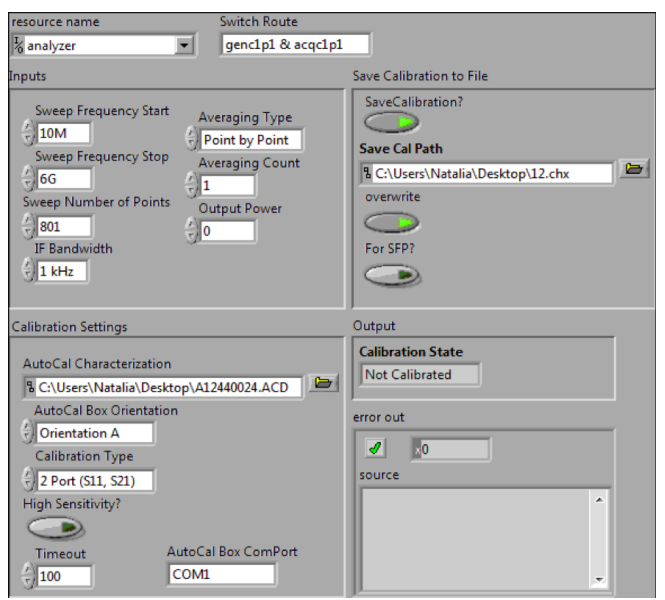

(a)

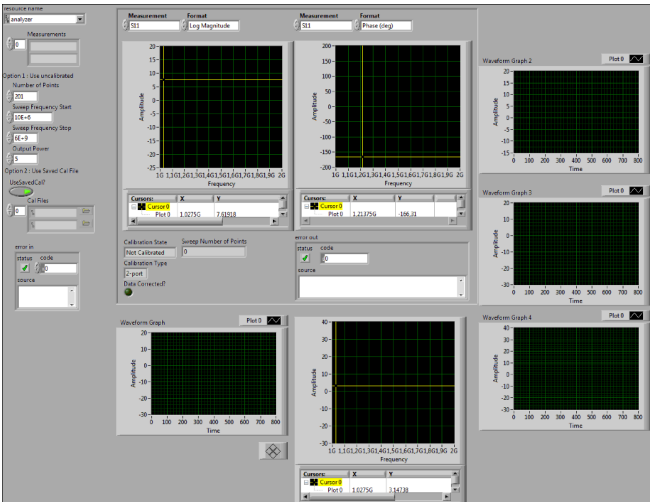

(b)

Figure 3.27: (a) Front panel for the calibration at the end of the cables of the switching network. (b) Front panel for the $S$ parameter measurement using the VNA NI PXIe-5632.

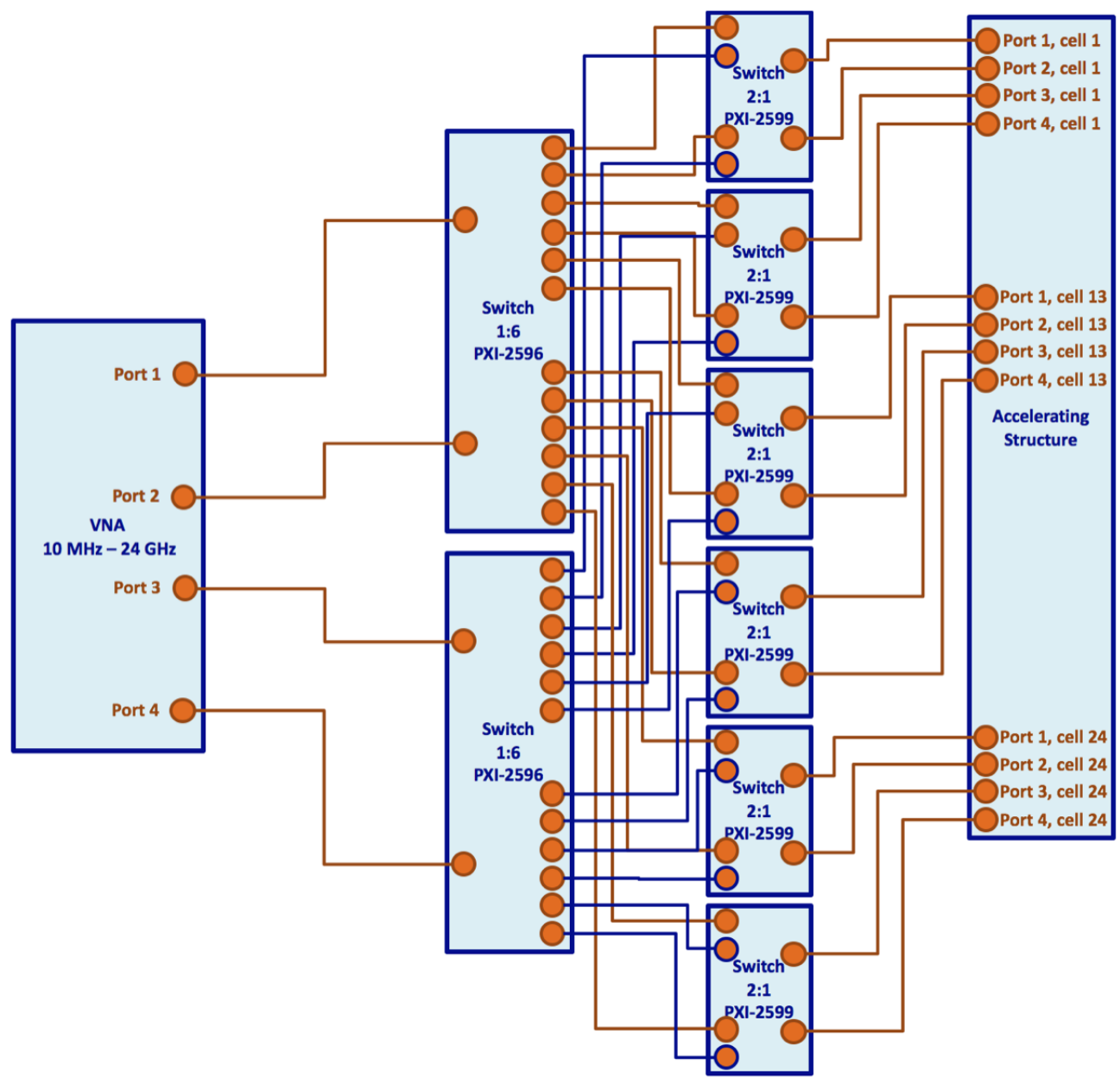

Figure 3.28: Switching network solution with equipment from National Instruments to extend a four-port VNA to twelve ports. 


\subsubsection{Development of a test bench to measure the EM axis of the TD24 par- allel to the beam direction}

A conceptual design of a second test bench compatible with the FPAB to measure the EM axes in the TD24 with the AS positioned horizontally, has been developed within Workpackage 4 of the PACMAN project in collaboration between ESR4.1 and ESR4.2 [50] [51] [97]. The aim is to localise the EM axes in three degrees of freedom using the same measurement method, and reproduce the positioning of the AS in the girder to be transported to the CLIC tunnel. This way, the AS would be placed in the direction of the beam and wakefields would be minimised inside the AS. The design of the test bench using CATIA has been registered under CERN EDMS number CLIBPMMB0031, and can be seen in Figure 3.29. The test bench consists of:

- Active optical table: An active-stabilised optical table model M-VIS3048-PG2$325 \mathrm{~A}$ [98], from Newport with a capacity of $590 \mathrm{~kg}$, was purchased in order to damp external vertical and horizontal vibrations by $85 \%$ or more above $5 \mathrm{~Hz}$ and by greater than $95 \%$ above $10 \mathrm{~Hz}$.

- Aluminium base: A rectified aluminium base allows the assembly of the bench elements to the optical table.

- Hexapod: The controlled-remote hexapod HXP100-MECA [99] from Newport is a six-axis translation stage that allows the movement of the AS in 6 degrees of freedom with sub-micron resolution steps. The resolution of the uni-directional repeatability is $(\mathrm{x}, \mathrm{y}, \mathrm{z})=(0.5,0.5,0.25) \mu \mathrm{m}$ and it is in the bi-directional repeatability $(\mathrm{x}, \mathrm{y}, \mathrm{z})=(4,4,2) \mu \mathrm{m}$, for a maximum load capacity of $20 \mathrm{~kg}$. Only 4 degrees of freedom are useful to localise the EM centre: the two axes perpendicular to the beam directions and the respective angles. The validation process of the specifications was done in the Leitz Infinity CMM in a set-up consisting of five spherical targets, mounted on the hexapod plate shown in Figure 3.30. The position of the hexapod was determined by interpolating a plane between the outer spheres of the plate and measuring the position of the sphere in the centre. The movement of the hexapod on the vertical axis with a step size of $5 \mu \mathrm{m}$ confirmed the uni-directional repeatability specification corresponding to the CMM uncertainty, Figure 3.31, while the bi-directional was failed. This last result indicates that the measurement algorithm should exploit the uni-directional movement of the hexapod or, in other words, the AS should be moved from a given starting point and step-size in the two scanning dimensions.

- Aluminium case: An aluminium case hosts the AS horizontally and is provided by screws, fixing the AS to the case.

- AS base: An aluminium base allows the fixation of the aluminium case to the hexapod.

- Wire supports with stretched-wire tools: Two wire supports with stretched-wire tools are fixed on the optical aluminium base in order to grab and stretch the $\mathrm{Be}-\mathrm{Cu}$ wire of $0.1 \mathrm{~mm}$ of diameter at both extremes of the AS. 
Chapter 3: Design, fabrication and calibration of an experimental set-up to measure the

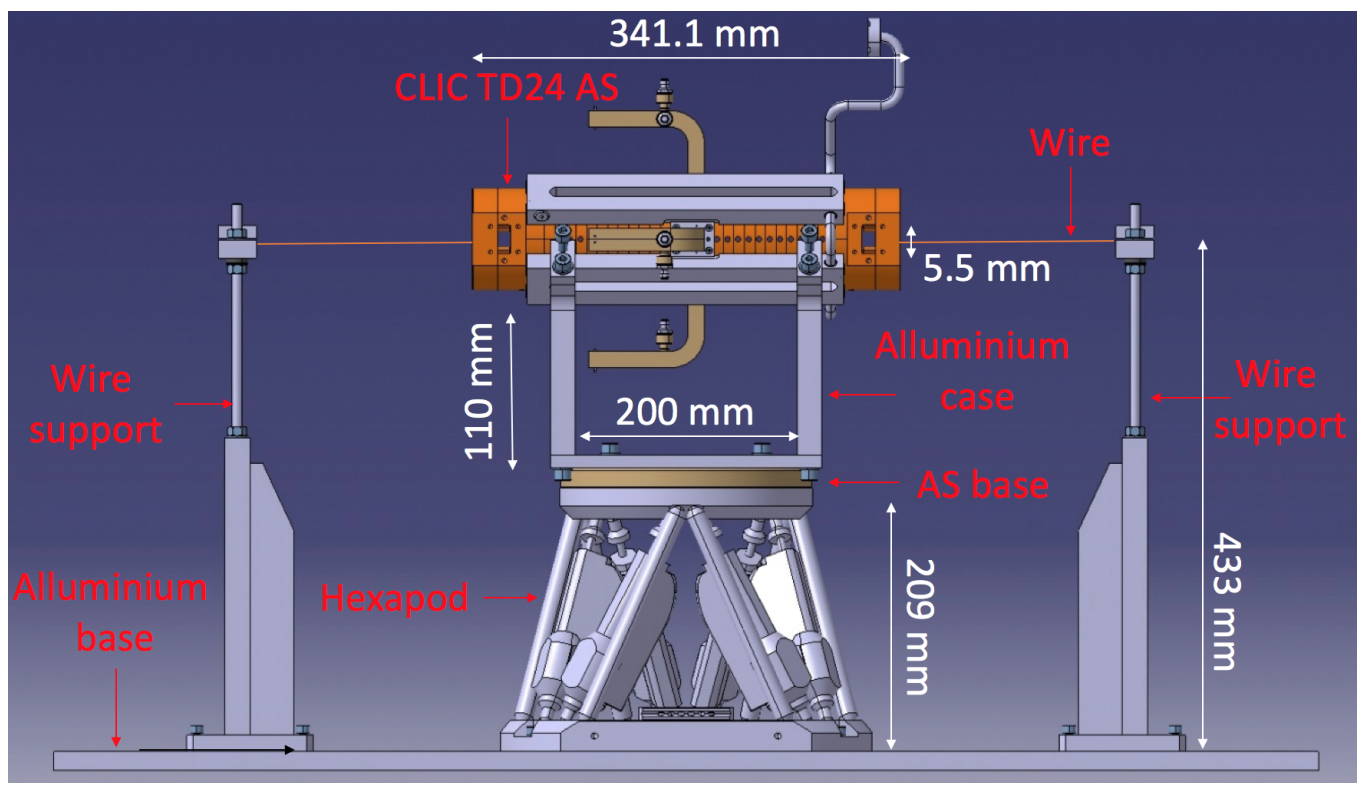

Figure 3.29: Test bench designed for the measurement of the EM axis in the middle disk of the TD24 when the AS is placed horizontally.

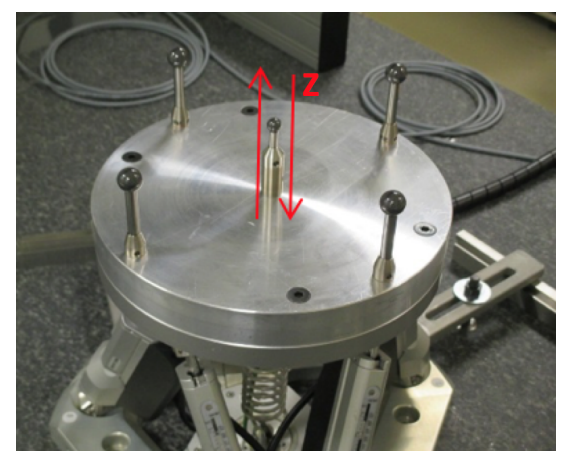

Figure 3.30: Validation of the hexapod specifications in the Leitz Infinity CMM.

- 4-port VNA: The same VNA, as already explained, can be used in order to perform the $S$ parameter measurements in order to localise the EM axis in the middle cell of the TD24.

- User platform: A user platform can be developed using LabVIEW to control the motion of the hexapod, perform automatic measurements with the VNA as well as data processing and analysis.

Further developments to ease the installation of the wire through the AS need to be performed. The current process consists of a rotation of the AS in order to position it vertically, install the wire with gravity and rotate the AS again for its horizontal positioning in the AS case. This technique is very complicated given the weight of the AS, that is difficult to handle. Thus, a mechanical solution with the AS fixed in the horizontal position should be investigated to pass the wire through the AS without touching the small-aperture irises with a mean diameter of $5.5 \mathrm{~mm}$. In addition to that, a mechanical solution needs to be investigated for the minimisation of the wire sag. 


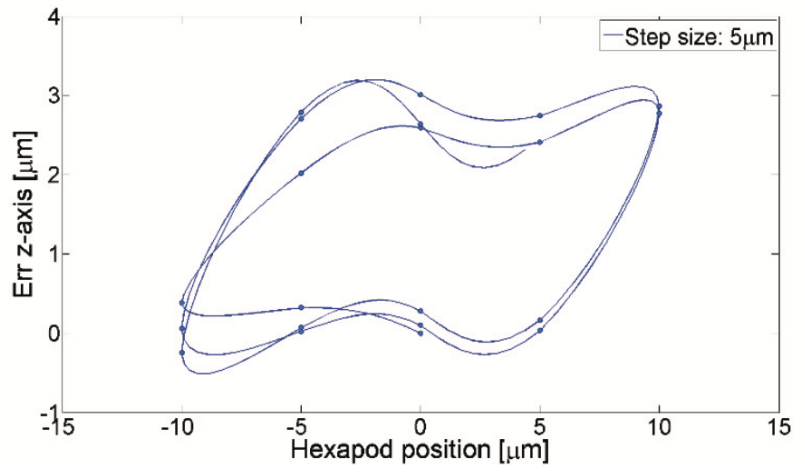

Figure 3.31: Measurement of the error on the positioning of the hexapod in the $Z$ direction. 
Chapter 3: Design, fabrication and calibration of an experimental set-up to measure the EM axes of the CLIC TD24 using the perturbative method 


\section{Chapter 4}

\section{Experimental measurements of the EM axes of the TD24 and analysis using the perturbative method}

A new methodology to measure the EM axes of ASs, based on the Slater perturbation theorem, has been presented in Chapter 2 and has been referenced in this thesis as the perturbative method. The implementation of this method has been explained in detail in Chapter 3, giving as a result a test bench that we will use in this chapter to measure the EM axes of the TD24.

In this chapter, we will describe first, the experimental proof-of-principle of the perturbative method using the WFM already assembled to the middle cell of the TD24, following Figure 4.1 (a). Secondly, taking into account the results previously obtained with the WFM, we decide to improve the measurement by replacing the WFM with tapered transitions, following Figure 4.1 (b). In a third step, virtual ports have been added to the measurement to improve the performance. Finally, the experimental results have been analysed and compared with simulations. Furthermore, absolute positioning measurements of the AS with respect to the wire have been performed to crosscheck the repeatability of the measurements, and other experimental tests have been made striving to identify and characterise possible sources of errors.

\subsection{Experimental proof-of-principle with WFM signals}

At the initial stage of the bench development phase, we performed an experimental proofof-principle of the perturbative method using the WFM available at that time and assembled to the middle cell of the TD24 [97] [101] [55]. We first wanted to demonstrate the linear dependance of the wire displacement with the power signals in the middle cell, following equations from (2.59) to (2.62). Secondly, we wanted to confirm the choice made for the reference frequency via simulations. And finally, we will optimise time and resources in the computation of the EM centre. 


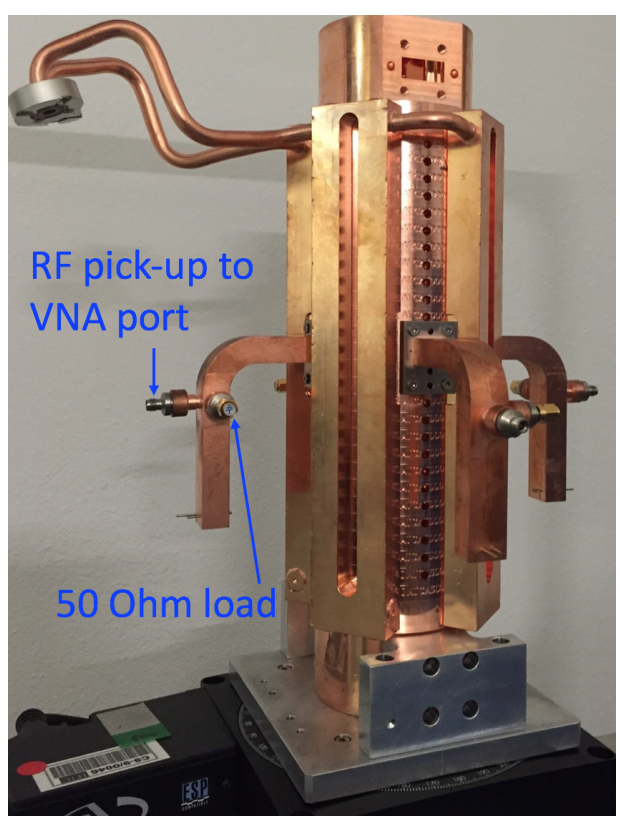

(a)

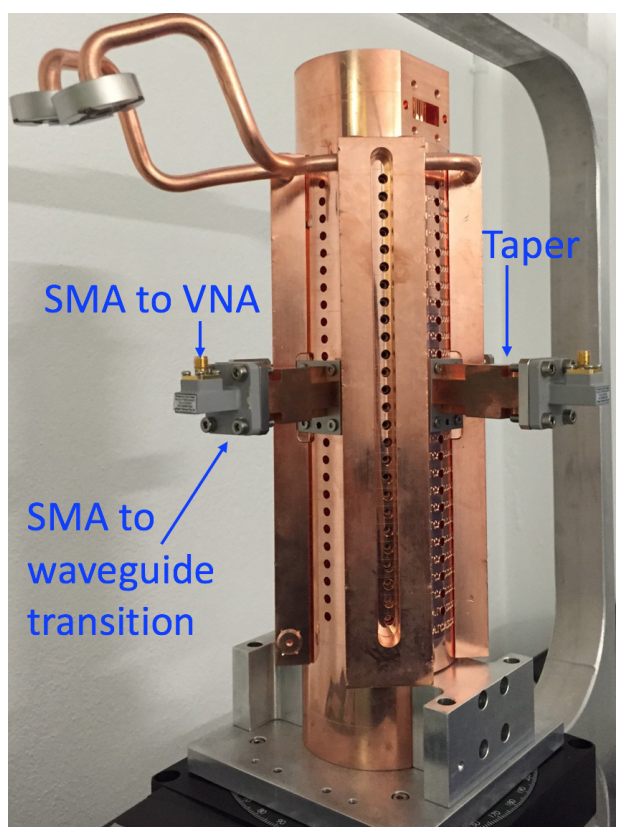

(b)

Figure 4.1: (a) WFM assembled to the middle cell of the TD24. (b) Tapered transitions assembled in the middle cell in the subsequent measurements of the EM centre.

We connected the four RF pick-ups designed to couple the high order dipole mode at $18 \mathrm{GHz}$ to the VNA ports. The other four RF pick-ups, coupling to the high order monopole mode at $24 \mathrm{GHz}$, were terminated with a $50 \mathrm{Ohm}$ load to avoid reflections. No calibration on the wire perpendicularity was performed as the wire positioning systems were missing at that time. Furthermore, no calibration of the instrument equipment was done, due to the non availability of an appropriate SMA calibration kit in the laboratory. Once we proceeded with the installation of the PACMAN wire, we performed the following experiments:

\section{Identification of the experimental reference frequency.}

We moved the linear stage in $X$ in a long range of $6 \mathrm{~mm}$ with a coarse step size of $100 \mu \mathrm{m}$. All the $S$ parameters were measured at each position in a frequency range from 16 to $23 \mathrm{GHz}$. In Figure 4.2 the asymmetry measurement from p2 defined in Chapter $2 A M_{2}$, is plotted as a function of the frequency for different wire positions in $X$. The different colour traces represent a different wire position. The plot only shows the results when exciting the cell from $\mathrm{p} 2$, but the results for $A M_{4}$ are equivalent. The reference frequency was obtained as the most sensitive to the wire displacement through the observation of the plot. The most sensitive frequencies to the wire displacement and with minimum noise are flagged with blue circles, and are around $17 \mathrm{GHz}, 18 \mathrm{GHz}$ and $18.5 \mathrm{GHz}$. In all three cases, $S$ parameters change monotonically with the wire position. However, the first one, around $17 \mathrm{GHz}$, gives a larger response. This confirms the EM field simulation results whose reference frequency was also found at $17 \mathrm{GHz}$. From now on, we will measure the EM centre at this frequency. 


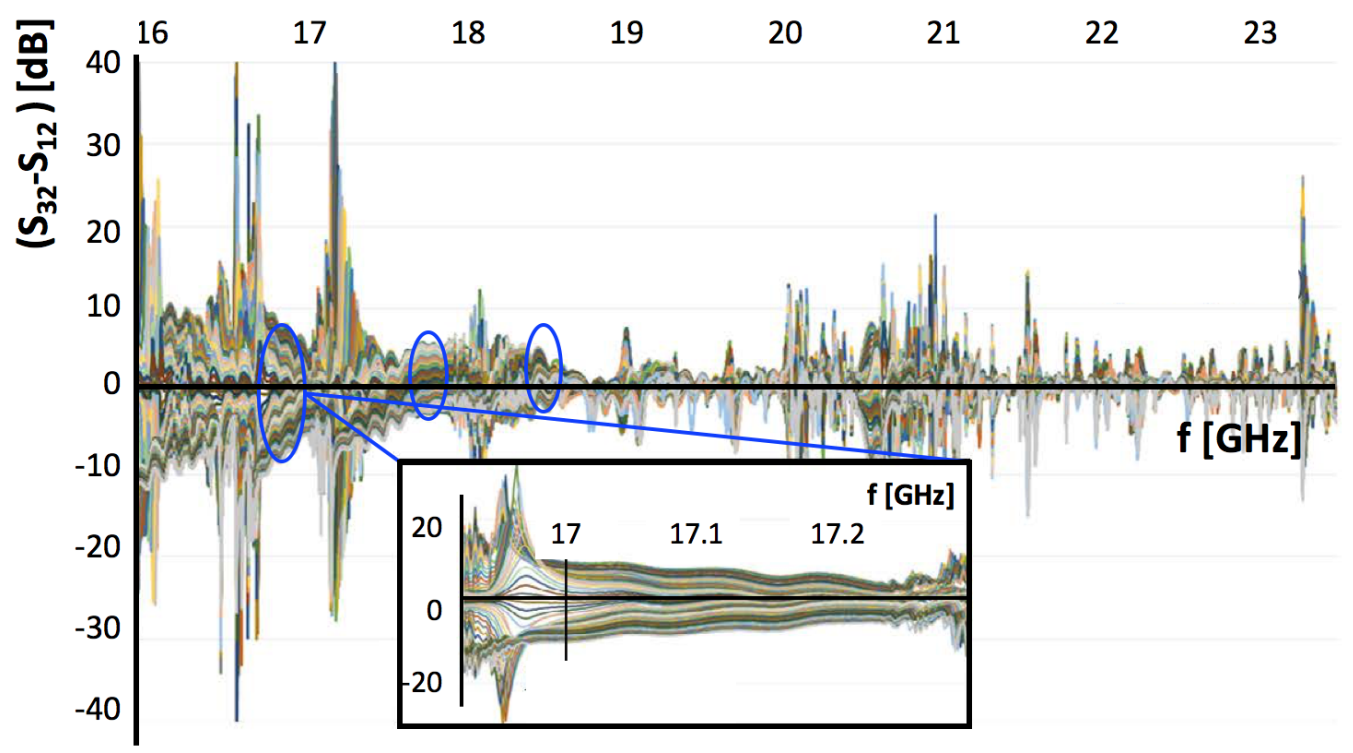

Figure 4.2: Asymmetry measurements with respect to the frequency when the AS moves along $X$ while $Y$ is fixed at the home position. The different colours represent the different wire positions. The blue circles indicate the most sensitive frequencies to the wire displacement and with minimum noise. The figure in the bottom represents a zoom of the first pointed frequency range.

\section{First experimental determination of the EM axes.}

Once the selection of the reference frequency has been done $(17 \mathrm{GHz}), A M_{2}$ (green dots) and $A M_{4}$ (red dots) are represented as a function of the position of the linear stage in $X$, as shown in Figure 4.3. Similarly, we represent $A M_{1}$ (red dots) and $A M_{3}$ (green dots) when moving in the $Y$ axis in Figure 4.4. We expected a linear relationship around the position of the EM centre. We can distinguish in Figure 4.3 (a) a linear area in zone II, while in zones I and III the experimental measurements seem to saturate. Since the AS moves in a range bigger than the iris aperture, the wire touches the iris walls and can no longer be moved. This will explain that the values of $\left\{A M_{1}, \ldots, A M_{4}\right\}$ are constant beyond this point. As we saw in Chapter 2, the asymmetry measurements are zero when the wire is positioned at the EM centre of the AS. To improve precision, we linearly fit the measured points and we calculate the value at which the fitted line crosses zero. This value could be taken as the $X$ coordinate of the EM centre of the AS. The goodness of the linear fit, or the coefficient of determination, is given by $R^{2}$ which is defined as:

$$
R^{2}=1-\frac{\sum_{i}\left(y_{i}-g_{i}\right)^{2}}{\sum_{i}\left(y_{i}-\bar{y}\right)^{2}}
$$

where $\bar{y}$ is the mean of the observed data with $n$ values $\left\{y_{1}, \ldots, y_{n}\right\}$ with an associated predicted value $\left\{g_{1}, \ldots, g_{n}\right\}$. Note that, as in simulations, we obtain two different centres, $X_{p i}, i=2,4$, with different values and different coefficients of determination: $X_{p 2}=-$ 
$0.5370 \mathrm{~mm}$ with $R^{2}=0.9988$ (Figure 4.3 (b)) and $X_{p 4}=-0.4934 \mathrm{~mm}$ with $R^{2}=0.9995$ (Figure 4.3 (c)). The same process is repeated in the $Y$ axis and the approximate position of the EM axes $(X, Y)$ is obtained. This corresponds, as explained in Chapter 3, with a full iteration of the algorithm. As fixed position for one axis, we used the arithmetic average of $X_{p i}$ as justified with simulations in Chapter 2. The asymmetry measurements from p1 and $\mathrm{p} 3$ can be seen in Figure 4.4. Two EM centres are also found in the $Y$ axis, $Y_{p j}$, $j=1,3$, with different coefficient of determination: $Y_{p 1}=-2.0809 \mathrm{~mm}$ with $R^{2}=0.9973$ (Figure 4.4 (b)) and $Y_{p 3}=-2.0617 \mathrm{~mm}$ with $R^{2}=0.9992$ (Figure 4.4 (c)).

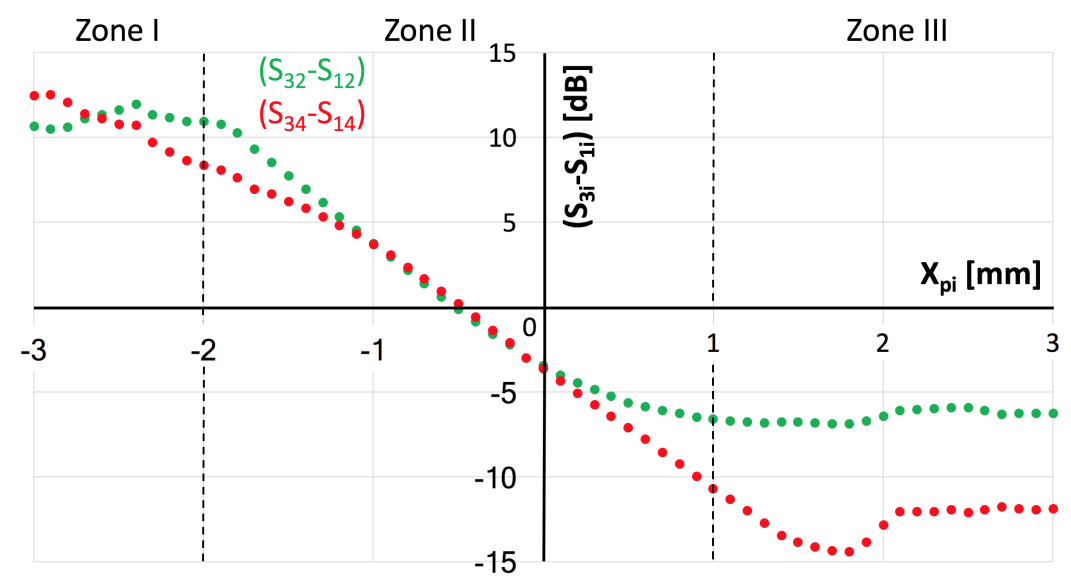

(a)

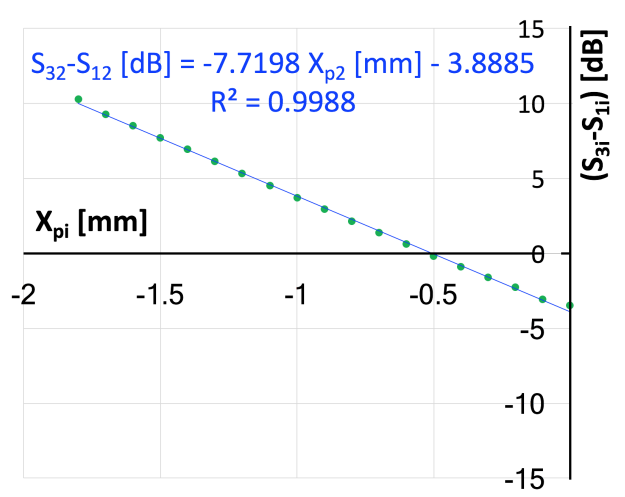

(b)

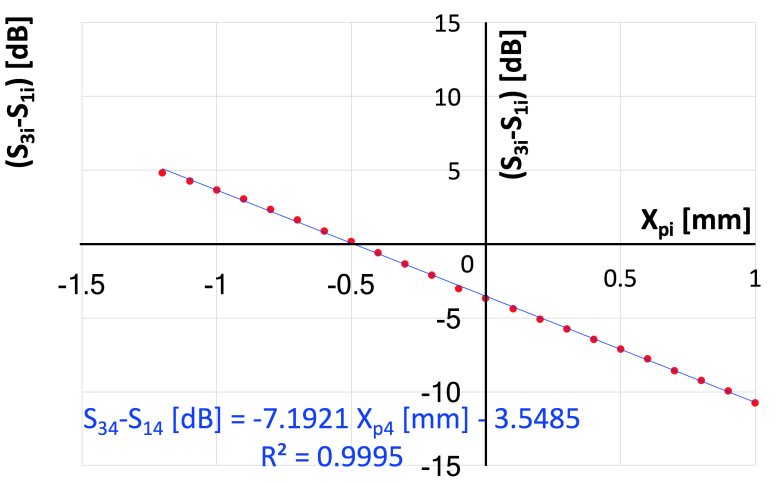

(c)

Figure 4.3: Asymmetry measurements (dots) with respect to the linear stage position in $X$. The AS was moved in a travel range of $6 \mathrm{~mm}$ with steps of $100 \mu \mathrm{m}$. The dash lines delimit the different zones distinguished in the plots. The zones I and III represent the non linear behaviour. The linear parts or zone II are represented in (b) $\left(S_{32}-S_{12}\right)$ and (c) $\left(S_{34}-S_{14}\right)$. The solid line represents the linear of $\left(S_{3 i}-S_{1 i}\right)$.

We iterated the same measurement in a shorter range, close to the previous computed centre and with smaller steps to further improve precision. Results of these measurements are shown in Figure 4.5, with an interval of $1 \mathrm{~mm}$ and $50 \mu \mathrm{m}$ step size; and in Figure 4.6 with an interval of $0.4 \mathrm{~mm}$ and $1 \mu \mathrm{m}$ step size. As we can observe in Figure 4.6, as we approach the required precision of around 1 or $2 \mu \mathrm{m}$, the signal to noise ratio increases and we need a large number of points to get a good fit, contrary to the simulation where 


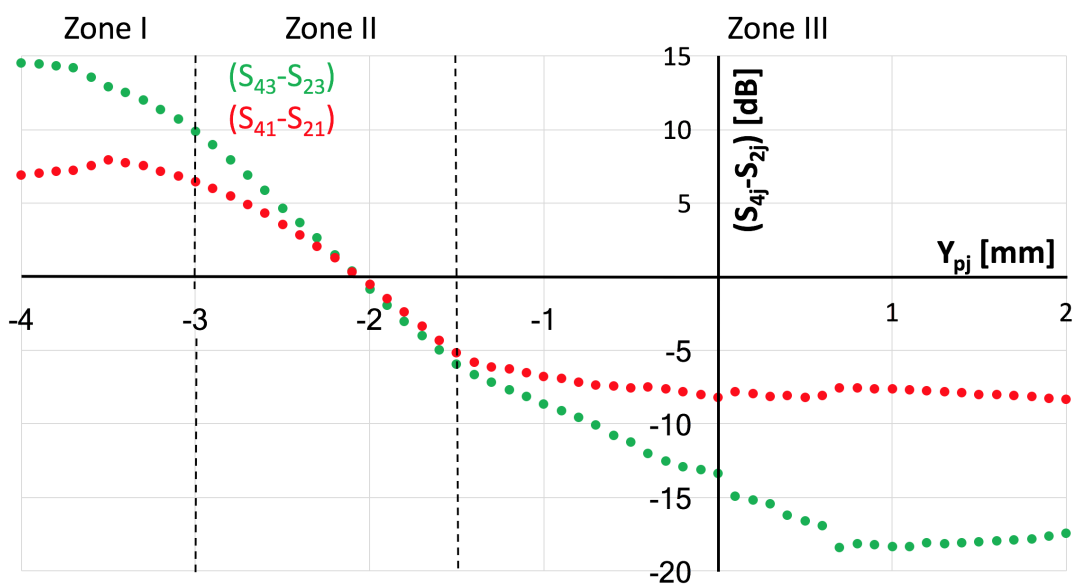

(a)

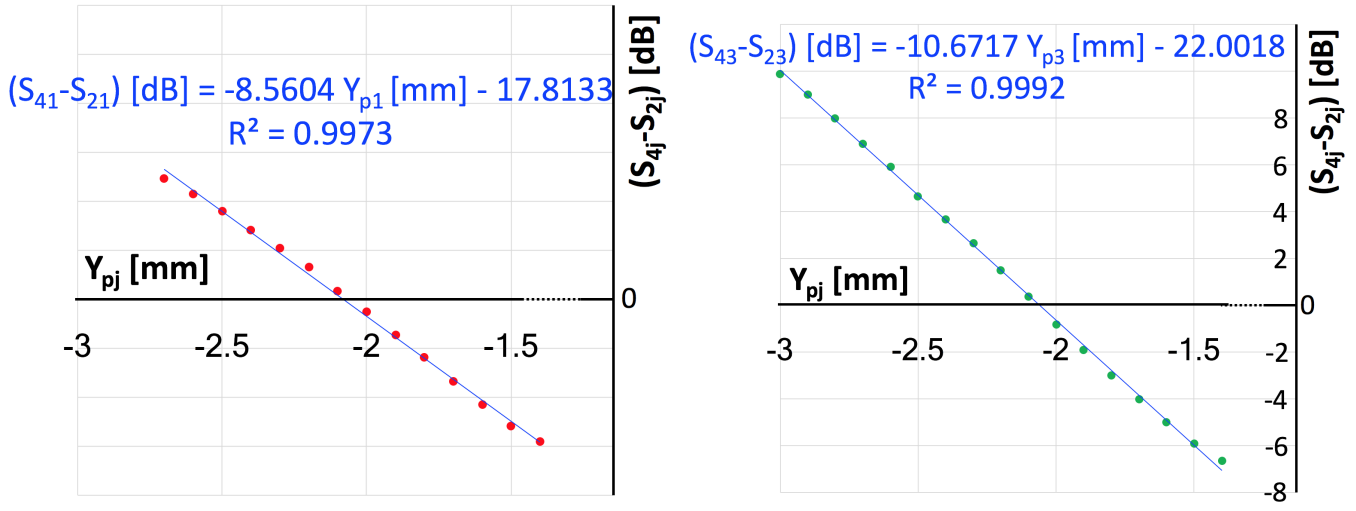

(b)

(c)

Figure 4.4: Asymmetry measurements (dots) with respect to the linear stage position in $Y$ and linear fit (solid line). The AS was moved in a travel range of $6 \mathrm{~mm}$ with steps of $100 \mu \mathrm{m}$. The dash lines delimit the different zones distinguished in the plots. The zones I and III represent the non linear behaviour. The linear parts or zone II are represented in (b) $\left(S_{41}-S_{21}\right)$ and (c) $\left(S_{43}-S_{23}\right)$.

only 9 points were needed.

In Figure 4.7, we represent the position of the EM centre in both axes at every iteration $N$. We observe that, after the first iterations, the centre as measured by each port converges very rapidly into a unique value. However, we found a different value of the centre of around $18 \mu \mathrm{m}$ in the $X$ axis and around $5 \mu \mathrm{m}$ in the $Y$ axis. This difference is much larger than what was anticipated by the simulations $( \pm 7.5 \mu \mathrm{m})$ and may be due to the lack of matching between the WFM and the middle cell of the TD24. Indeed, the WFM ports were designed to extract HOM power signals and not to input RF signals, so we decided to implement the manufactured tapered transitions with low reflection coefficient to recover the symmetry in the cell. We expect also to potentially improve the signal to noise ratio due to the power absorbed by the RF loads of the WFM next to the input RF pick-up. Different sensitivities were also found depending on the port and plane even around the established EM centre: around $9 \mathrm{~dB} / \mathrm{mm}$ at $\mathrm{p} 1$ and $\mathrm{p} 4$, around $11.2 \mathrm{~dB} / \mathrm{mm}$ at $\mathrm{p} 3$, and 


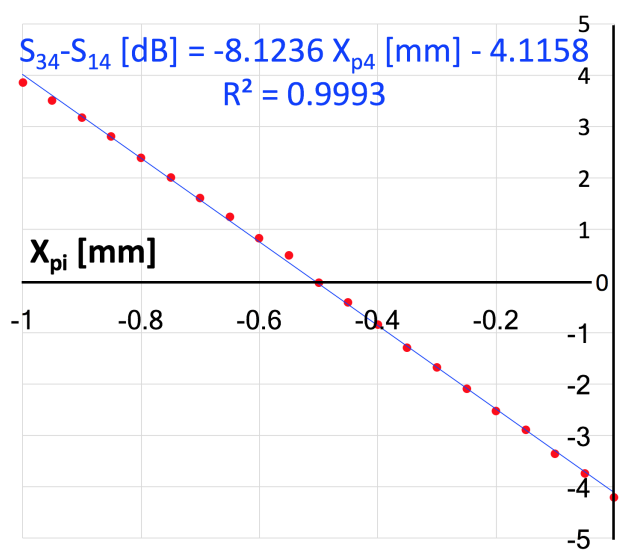

(a)

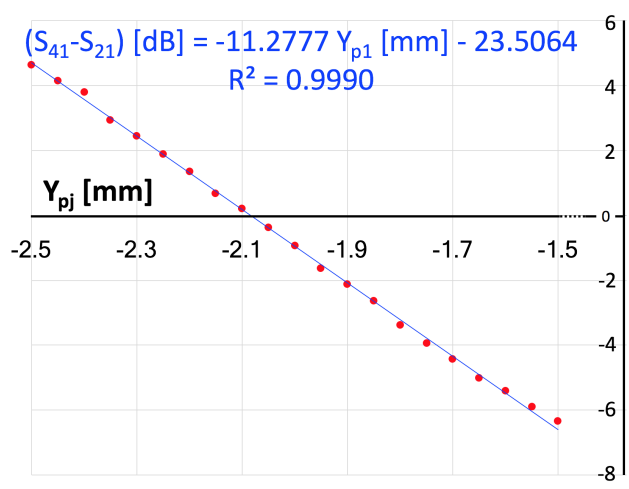

(c)

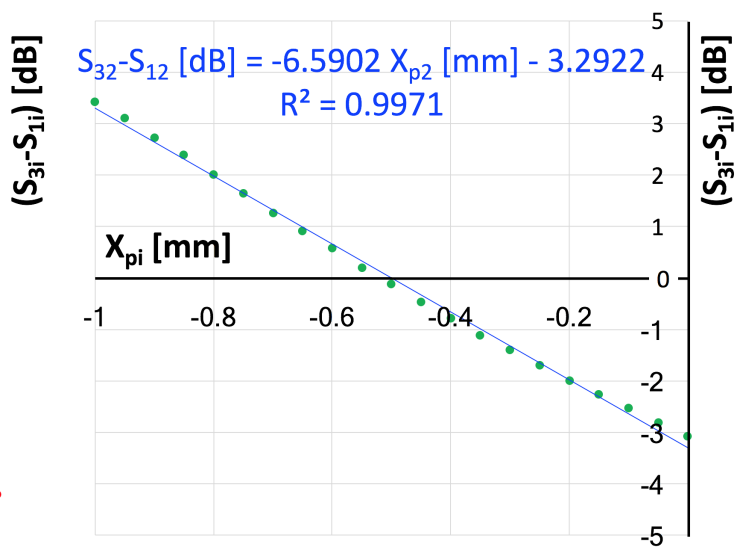

(b)

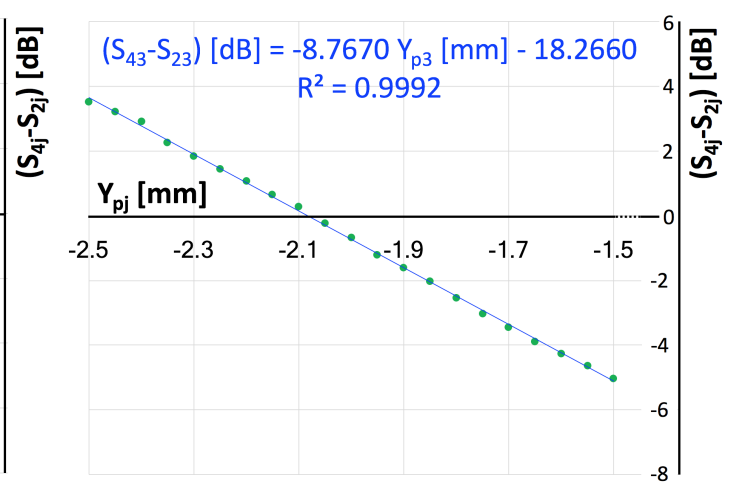

(d)

Figure 4.5: Asymmetry measurements (dots) with respect to the linear stage position in $X$ and $Y$ and linear fit (solid line). The AS moved in a travel range of $1 \mathrm{~mm}$ with steps of $50 \mu$ m. (a) $\left(S_{32}-S_{12}\right)$ (b) $\left(S_{34}-S_{14}\right)$ (c) $\left(S_{41}-S_{21}\right)$ (d) $\left(S_{43}-S_{23}\right)$.

around $6.2 \mathrm{~dB} / \mathrm{mm}$ at $\mathrm{p} 2$. Comparing with simulations in Chapter 2 (section 2.4.3.2), we could say that this is most probably due to the wire tilt that was not calibrated at that time.

\section{Resolution study.}

In order to optimise time and resources, we analysed the minimum travel range and maximum step size of the AS that lead to the same result for the EM centre position without significant loss of precision. For this purpose, we used the data set of a measurement from one port in which the AS was moved along $0.4 \mathrm{~mm}$ with steps of $1 \mu \mathrm{m}$. The position of the EM centre computed with the full set of data, $X_{r e f}$, was chosen as the reference. Data sub-sampling corresponding to travel ranges of 0.2 and $0.1 \mathrm{~mm}$ were also considered with step sizes ranging from 1 to $20 \mu \mathrm{m}$. The results of the EM centre error in each case with respect to the reference $\Delta X_{\text {ref }}$ are plotted in Figure 4.8. The blue, green and red bars correspond to a travel range of $0.1,0.2$ and $0.4 \mathrm{~mm}$, respectively. As we want to keep the final result below 1 or $2 \mu \mathrm{m}$, we decided that, for the next experiments, the AS will move in steps of $2 \mu \mathrm{m}$ in a travel range of $0.1 \mathrm{~mm}$. 


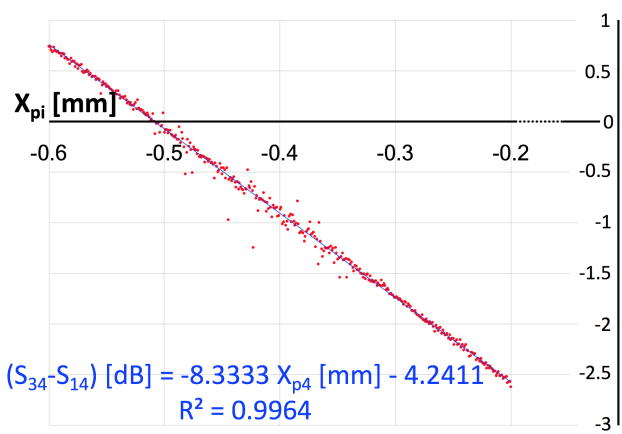

(a)

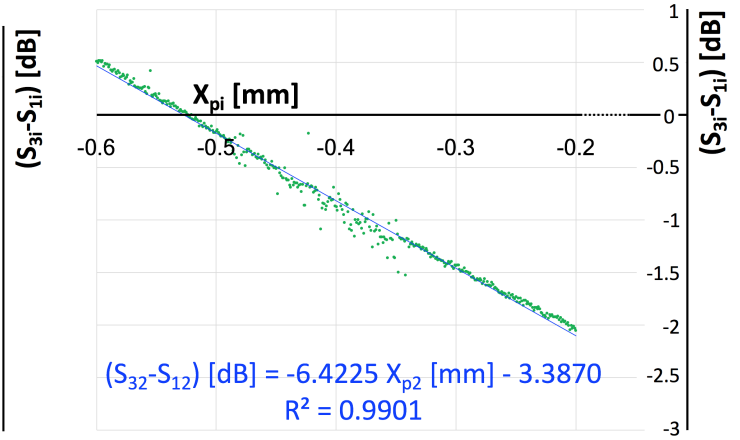

(b)

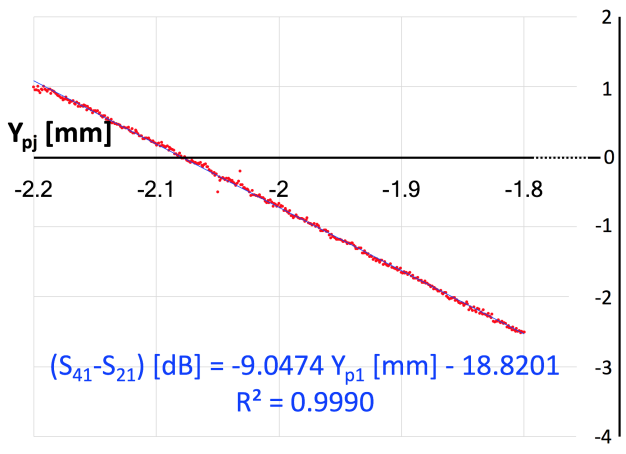

(c)

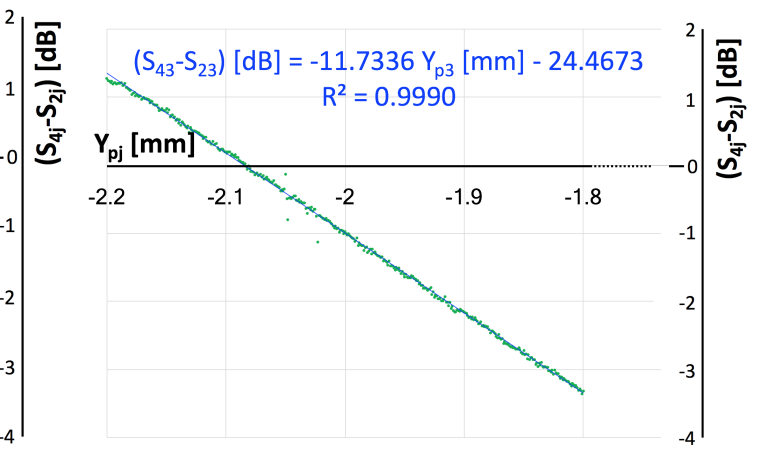

(d)

Figure 4.6: Asymmetry measurements (dots) with respect to the linear stage position in $X$ and $Y$ and linear fit (solid line). The AS moved in a travel range of $0.4 \mathrm{~mm}$ with steps of $1 \mu$ m. (a) $\left(S_{32}-S_{12}\right)$ (b) $\left(S_{34}-S_{14}\right)$ (c) $\left(S_{41}-S_{21}\right)(\mathrm{d})\left(S_{43}-S_{23}\right)$.

To summarise, the conclusions obtained from the first experiments using WFM signals are the following ones. First, we have identified the experimental reference frequency at $17 \mathrm{GHz}$, that coincides with the obtained via EM field simulation studies. After that, we have verified the good linear relationship between the wire position and the asymmetry measurements $\left\{A M_{1}, \ldots A M_{4}\right\}$ around the EM centre, given by the coefficient of determination approaching one, even with the use of WFM. We have also demonstrated that it is possible to locate the EM axes in the middle cell with a stretched wire along the TD24, and with four unbalance measurements using a VNA with very similar results. Their difference within the micron range, is accounted as the measurement error, $\xi_{u}$, and could potentially be improved with the implementation of tapered transitions with low reflections in the middle cell instead of using WFM. Finally, we found that time and resources could be optimised moving the AS in a minimum travel range of $0.1 \mathrm{~mm}$ with a maximum step size of $2 \mu \mathrm{m}$. 
Chapter 4: Experimental measurements of the EM axes of the TD24 and analysis using the perturbative method

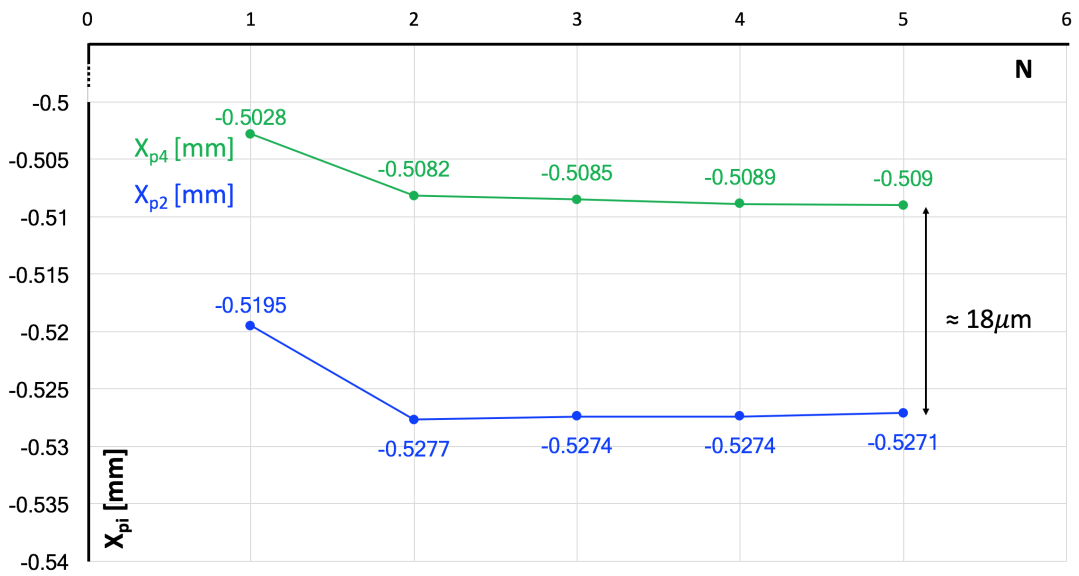

(a)

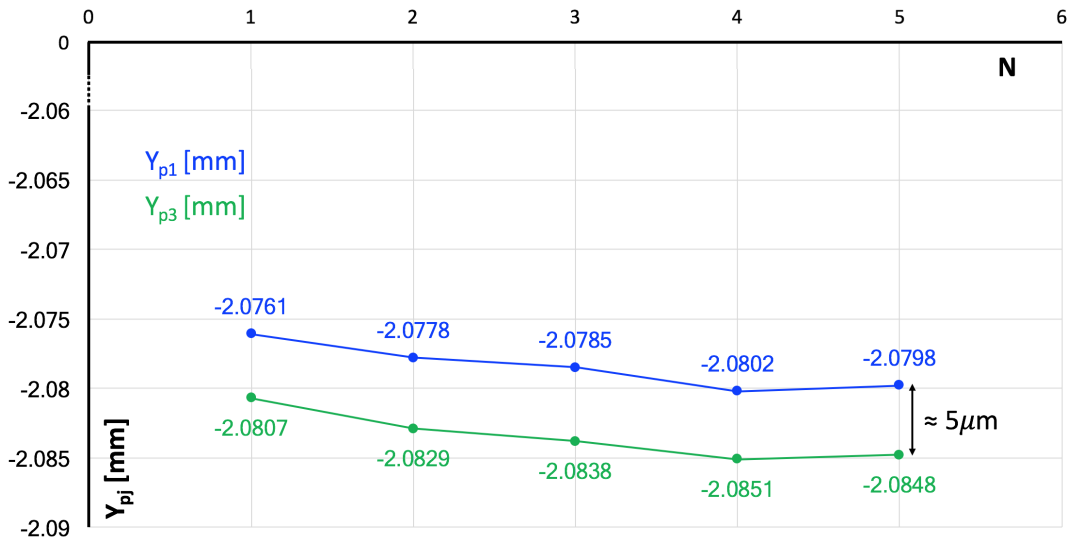

(b)

Figure 4.7: Position of the EM centre found in the $X$ (a) and $Y$ (b) axes with respect to the iteration number $N$. The AS moved in a range of $0.4 \mathrm{~mm}$ along both axes with a step size of $1 \mu \mathrm{m}$.

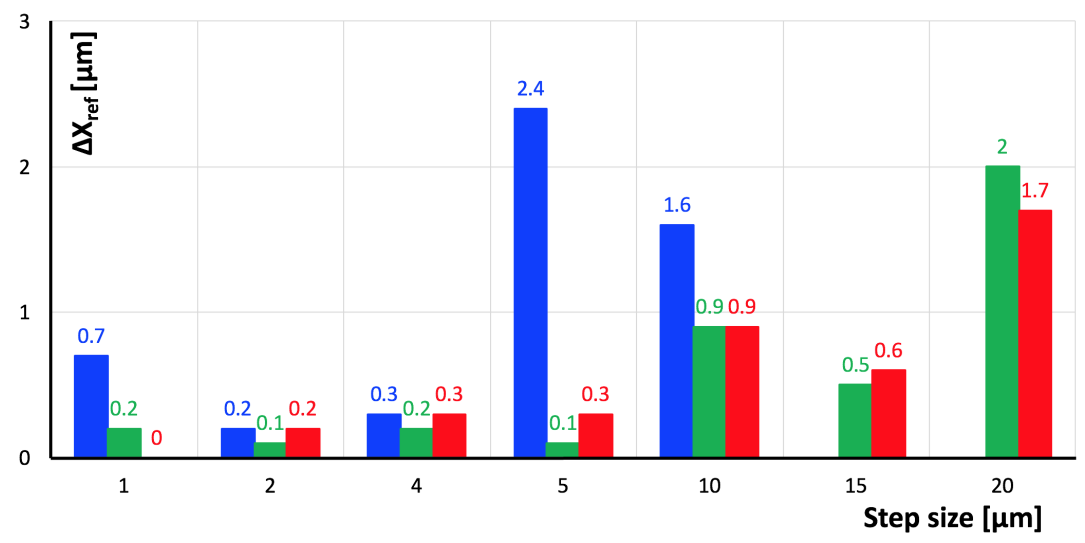

Figure 4.8: EM centre deviation with respect to the step size. The blue, green and red bars correspond to a travel range of $0.1,0.2$ and $0.4 \mathrm{~mm}$, respectively. 


\subsection{Experimental measurements of the EM axes with tapered transitions}

In this section, we aim to improve the EM centre measurements using VNA calibrations and manufactured tapered transitions replacing the WFM, as pictured in Figure 4.1 (b). The design of the tapers could be seen in Chapter 2 (2.4.3.3), and the manufactured parts in Chapter 3 (section 3.1). We will first verify the hypothesis that the wire perturbs minimally the AS when it is located in the centre of the cell. And later, we will determine the improvement of the EM centre measurement. The experimental procedure was done as follows:

Verification of minimum EM fields perturbation created by the wire positioned in the EM axis.

In this part, we want to verify that the perturbation created by the wire at the EM axes is minimum and similar to the unperturbed situation. Before installing the wire, we measured $\left(A M_{2, u n}\right)$ in a frequency range from 16 to $18 \mathrm{GHz}$. We installed the wire afterwards, and measured the EM centre following the same procedure as explained in the previous section with WFM. The AS moved in a travel range of $1 \mathrm{~mm}$ with steps of $5 \mu \mathrm{m}$. For each wire position in $X$, we obtained $A M_{2, X}$. In Figure 4.9, we present $A M_{2, X}$ (lines with different blue colour tones) with respect to the frequency for different wire positions in the $X$ axis and the unperturbed TD24, $A M_{2, u n}$ (green solid line). The red solid line represent $\left(A M_{2, X}\right)$ at the position of the EM coordinates in the $X$ axis, so $X=0$. We could observe that $A M_{2, X=0}$ is around zero from around 16.6 to $18 \mathrm{GHz}$ and very similar to $A M_{2, \text { un }}$, verifying the hypothesis of the perturbative method. The calibration of the VNA and the $S$ parameter measurement in linear form, are the responsible of a cleaner sensitivity of $A M_{2, X}$ compared to the measurement in Figure 4.2. The same sensitivity is obtained in both planes with a value in the linear form of $\frac{0.34}{1 \mathrm{~mm}}$.

\section{Measurement of the EM centre using tapered transitions and VNA calibration.}

We performed several measurements of the EM centre in order to evaluate its precision, defined as the standard deviation of converging measurements. The number of algorithm iterations was changed in each measurement. The results are shown in Figure 4.10 where precisions between 1 to $5 \mu \mathrm{m}$ for the vertical and horizontal planes are obtained, respectively. Note that an asymmetric difference between the measured centres at each plane still exists. The different precisions plotted at each $M-n$, also seems to be proportional to the number of algorithm iterations. In addition to that, the position of the EM centre as measured by each port differs around $20 \mu \mathrm{m}$, but precision has improved with respect to the WFM signals.

To conclude, we have verified that we are able to localise the position where the perturbation created by the wire inside the TD24 is minimised, and coincides with the unperturbed case. Furthermore, the VNA calibration and tapered transitions have improved the EM centre precision. The centre is measured with precisions between 1 to $5 \mu \mathrm{m}$ in the vertical and horizontal planes, respectively. The tapered transitions that we designed and manufactured with low reflection coefficient should have allowed us to recover the 
symmetry. However, asymmetry was still present between ports and between planes, and further investigations of its origin needs to be performed. At that time, the systematic errors were still unknown and an error of around $\pm \mathbf{1 0} \mu \mathbf{m}$ was obtained. Its reduction was expected with the further wire tilt calibration of the test bench.

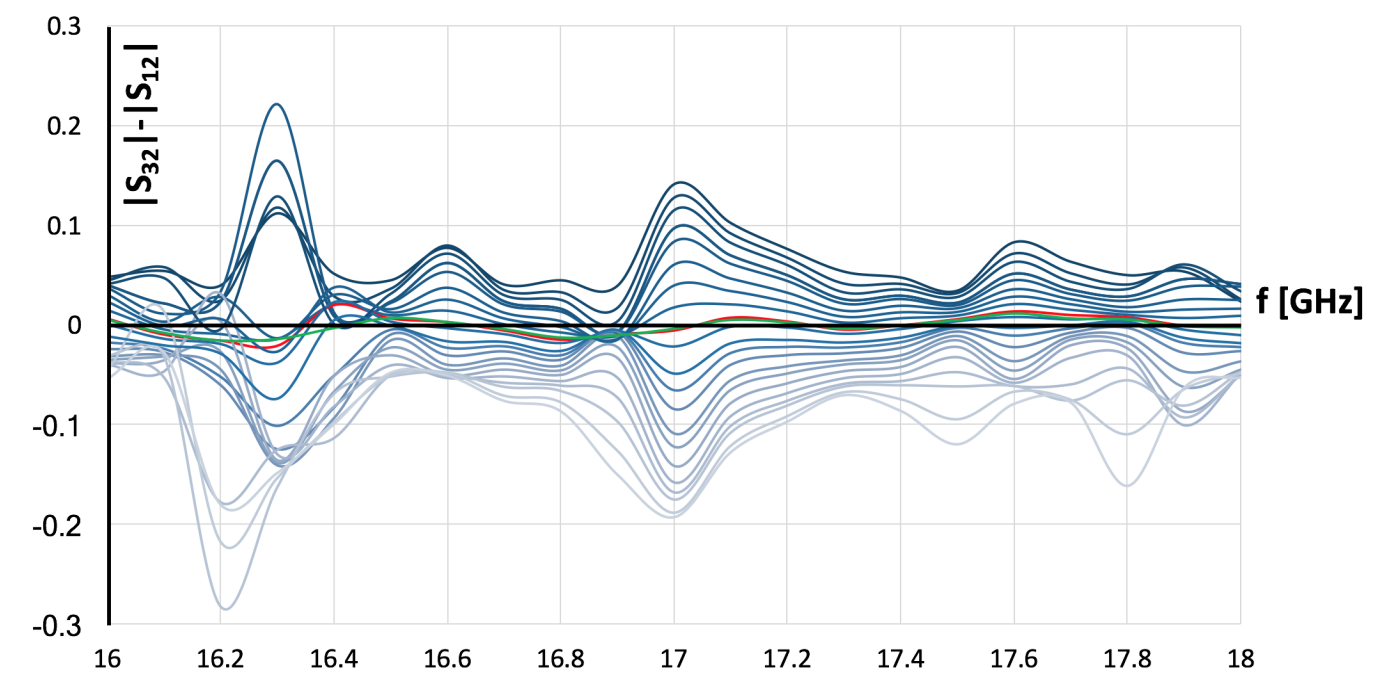

Figure 4.9: Asymmetry measurements in linear form (Eq (2.53) in Chapter 2) with respect to the frequency when the AS moves along $X$ while $Y$ is fixed at the home position. The different blue colours represent the different wire positions, while the red line represents the case when the wire is positioned at the $X$ coordinates and the green line represents the result of the unperturbed AS.

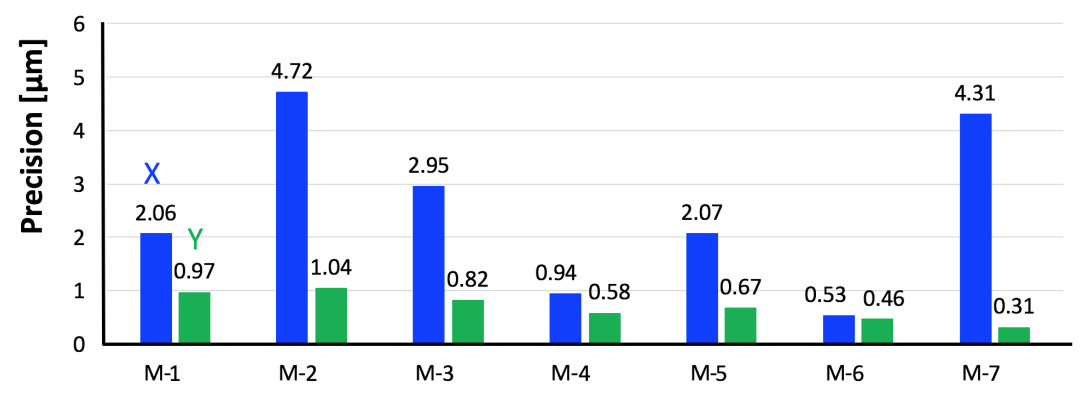

Figure 4.10: Precision of the EM centre with respect to the measurement number (M-n), measured with tapered transitions and a calibrated VNA. The blue bars represent the $X$ axis and the green bars, the $Y$ axis. 


\subsection{Algorithm optimisation and final results}

In this section, we aim to reduce the difference between the two centres found with asymmetry measurements, $\left\{A M_{1}, \ldots A M_{4}\right\}$, performed at each port. For this purpose, we investigated the possibility to measure the EM centre using one single balanced $S$ parameter measurement. Fortunately, at that time of the project, the wire-positioning system was available, and the fully assembly and calibrations of the elements and instrument equipment of the test bench was performed, following the descriptions in Chapter 3 [102] [17]. Therefore, we were in conditions to determine the final precision and repeatability of the EM centre measurement. The results will be shown in the last part of this section.

\section{Investigation of balanced $S$ parameter measurements with virtual port configura- tion of the VNA}

We aim to determine if the asymmetry found when measuring the EM centre using the asymmetry measurements $\left\{A M_{1}, \ldots A M_{4}\right\}$ is given by the asymmetric EM field pattern created in the middle cell when exciting from one single port, as seen in Figure 4.11. For this purpose, we studied the possibility of exciting simultaneously two opposite ports and create a symmetric EM field pattern [103]. We thought that this could be done using the virtual port configuration option of the VNA, but no further detail was given in the VNA specifications to verify this.

We examined the virtual port excitation of the VNA with an oscilloscope. Its four ports were connected to a four-channel oscilloscope with a bandwidth of $2 \mathrm{GHz}$ (model $\mathrm{R} \& \mathrm{~S}$ RTO1024 [100]) as indicated in Figure 4.11. The physical ports $\mathrm{p} 1$ and $\mathrm{p} 3$ were combined together as one virtual port (virtual B), and the same was reproduced for $\mathrm{p} 2$ and $\mathrm{p} 4$ (virtual A). We performed a test in which we excited virtual B with a single frequency signal at $100 \mathrm{MHz}$, with a span of $1 \mathrm{~Hz}$ and a power of $0 \mathrm{dBm}$ and obtained $\left|S_{3 B}\right|-\left|S_{2 B}\right|(\mathrm{Eq}(3.16))$. Later, we excited the same signal from virtual port A and obtained $\left|S_{3 A}\right|-\left|S_{1 A}\right|(\mathrm{Eq}$ (3.8)). The VNA output signals displayed in the oscilloscope are shown in Figure 4.12. A separate signal is observed from each port periodically with a duration of $1 \mathrm{~ms}$. In orange, the VNA excites $\mathrm{p} 1$ for $1 \mathrm{~ms}$; in pink, the excitation is done from $\mathrm{p} 3$; in blue, from $\mathrm{p} 2$ and in yellow, from $\mathrm{p} 4$. The plot shows that there is no simultaneous excitation of two ports when configuring the physical ports of the VNA as virtual ports. Indeed, this port configuration generates unbalance stimulus signals, and uses a mathematical transformation to convert unbalance wave quantities into balance $S$ parameters. So, the creation of a symmetric EM field pattern is not possible. However, we could still be able to recombine mathematically the two asymmetry measurements in each axis in one single measurement, very similar to the virtual port excitation of the VNA, as follows.

The $S$ parameters measured with a virtual port configuration following Figure 4.11, are related with the $S$ parameter measurements in each physical ports as:

$$
\begin{aligned}
& \left|S_{3 A}\right|=\left|S_{34}\right|+\left|S_{32}\right| \\
& \left|S_{1 A}\right|=\left|S_{14}\right|+\left|S_{12}\right| \\
& \left|S_{3 B}\right|=\left|S_{41}\right|+\left|S_{43}\right|
\end{aligned}
$$




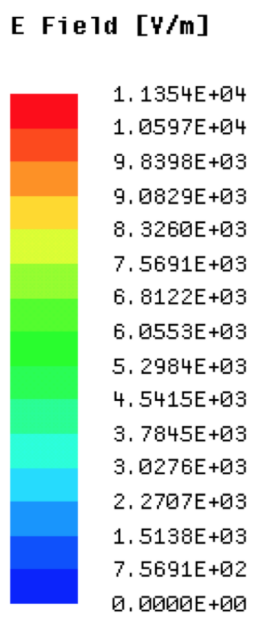

E Field [Y/m]

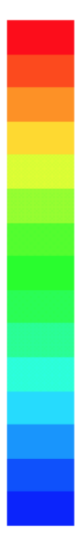

1. $1354 \mathrm{E}+04$

1. $0597 E+04$

9. $8398 \mathrm{E}+03$

9. $0829 \mathrm{E}+03$

8. $3260 \mathrm{E}+03$

7. $5691 \mathrm{E}+03$

6. $8122 \mathrm{E}+03$

$6.0553 \mathrm{E}+03$

5. $2984 \mathrm{E}+03$

4. $5415 \mathrm{E}+03$

3. $7845 \mathrm{E}+03$

3. $0276 \mathrm{E}+03$

2. $2707 \mathrm{E}+\square 3$

1. $5138 \mathrm{E}+03$

7. $5691 \mathrm{E}+\square 2$

ด. $\square 000 \mathrm{E}+\square 0$

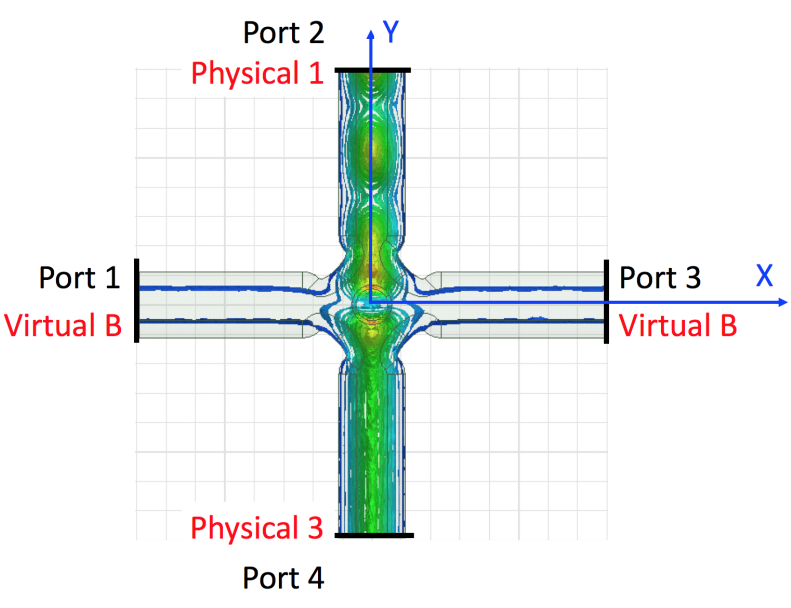

(a)

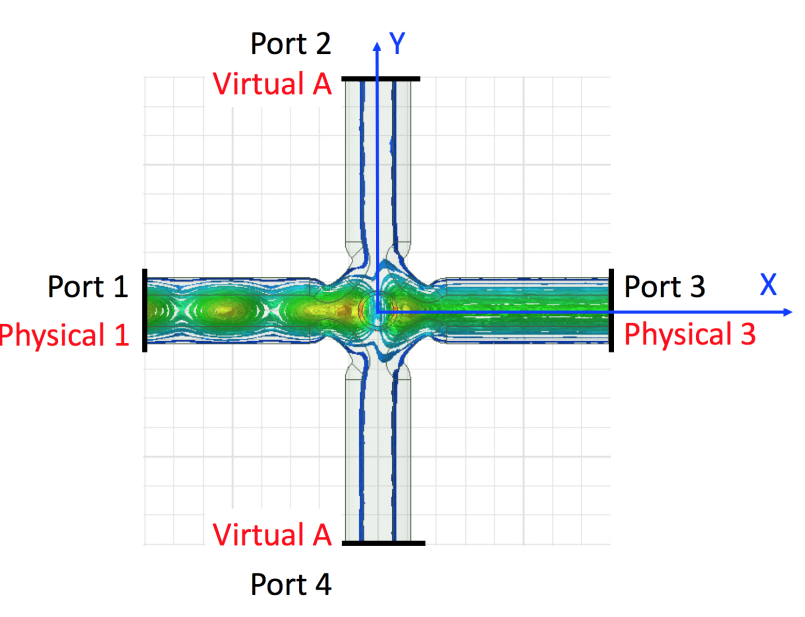

(b)

Figure 4.11: (a) Port connections in the middle disk of the CLIC TD24 accelerating structure when the linear stage in $Y$ is fix and the linear stage in $X$ moves. (b) Port connections in the middle disk of the CLIC TD24 accelerating structure when the linear stage in the $X$ is fix and the linear stage in $Y$ moves. 


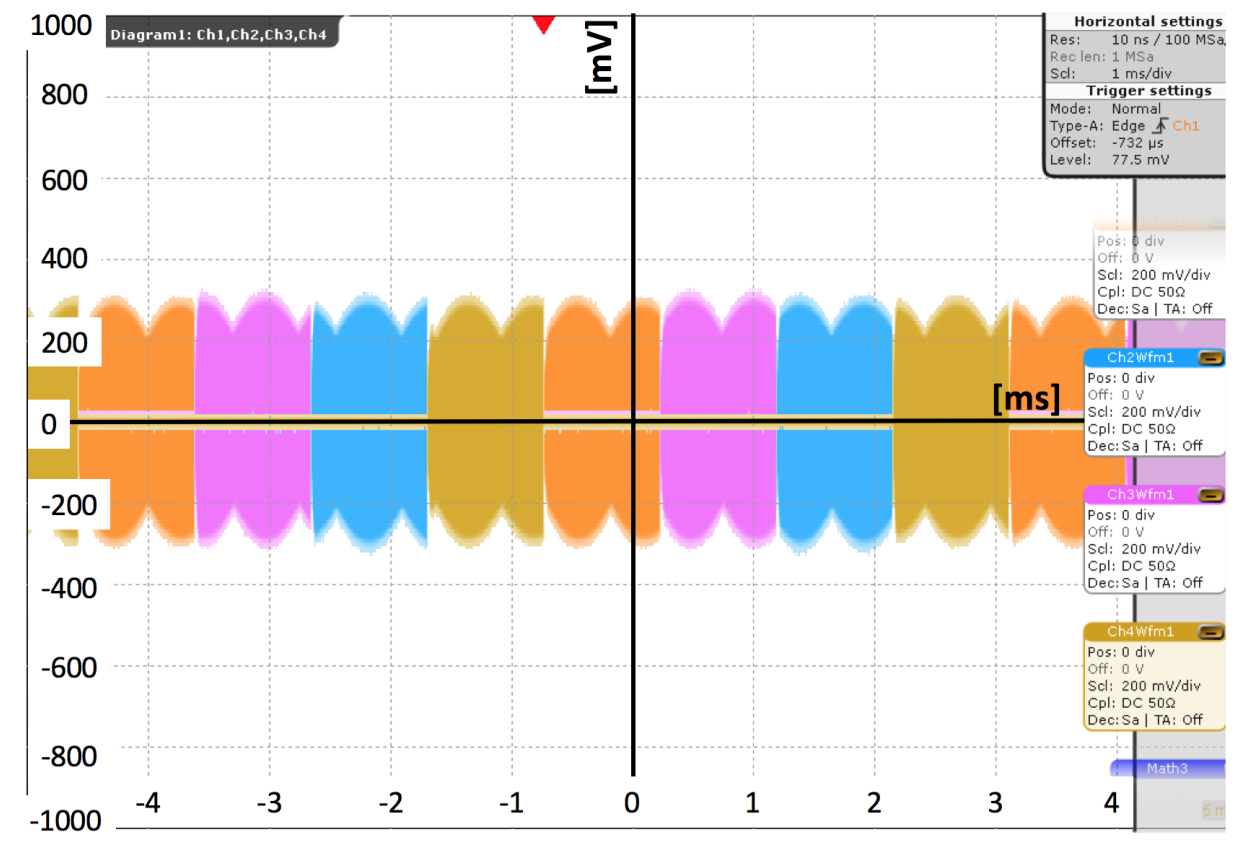

Figure 4.12: Voltage signals as a function of time displayed in the oscilloscope from the VNA. In orange, the VNA excitation from $\mathrm{p} 1$ is observed; in pink, the excitation is done from $\mathrm{p} 3$; in blue, from $\mathrm{p} 2$ and in yellow, from $\mathrm{p} 4$.

$$
\left|S_{2 B}\right|=\left|S_{21}\right|+\left|S_{23}\right|
$$

Thus, it is possible to compare the virtual port measurement with the arithmetic recombination of $\left\{A M_{1}, \ldots A M_{4}\right\}$ as:

$$
\left|S_{3 A}\right|-\left|S_{1 A}\right|(\mathrm{Eq}(3.7))=\left(\left|S_{32}\right|-\left|S_{12}\right|\right)+\left(\left|S_{34}\right|-\left|S_{14}\right|\right)(\mathrm{Eq}(3.6))
$$

when moving the wire along $X$ and:

$$
\left|S_{3 B}\right|-\left|S_{2 B}\right|(\operatorname{Eq}(3.15))=\left(\left|S_{41}\right|-\left|S_{21}\right|\right)+\left(\left|S_{43}\right|-\left|S_{23}\right|\right)(\operatorname{Eq}(3.14))
$$

when moving the wire along $Y$. Therefore, the position of the EM centre in $X$ could be obtained resolving independently:

$$
\left|S_{3 A}\right|-\left|S_{1 A}\right|=0(\mathrm{Eq}(3.11))
$$

and

$$
\left(\left|S_{32}\right|-\left|S_{12}\right|\right)+\left(\left|S_{34}\right|-\left|S_{14}\right|\right)=0(\mathrm{Eq}(3.10))
$$

and two centres are obtained $X_{p 2+p 4}$ and $X_{v 24}$. Applying the same when moving the AS along $Y$, we resolve:

$$
\left|S_{3 B}\right|-\left|S_{2 B}\right|=0(\mathrm{Eq}(3.19))
$$

and

$$
\left(\left|S_{41}\right|-\left|S_{21}\right|\right)+\left(\left|S_{43}\right|-\left|S_{23}\right|\right)=0(\mathrm{Eq}(3.14))
$$

and, two centres are obtained at $Y_{p 1+p 3}$ and $Y_{v 13}$. 
In Figure 4.13 (a) we present the convergence of the EM centre in the vertical axis computed as $\left(X_{p 2+p 4}, Y_{p 1+p 3}\right)$ with respect to the measurement number $N$. Similarly in Figure 4.13 (b), when the EM centre is calculated as $\left(X_{v 24}, Y_{v 13}\right)$. From the two measurements, we will select the less noisy as the position of the EM centre to iterate the algorithm. The virtual ports seem to introduce a bigger numerical noise, so we decided to end up with the algorithm shown in Figure 3.18 in Chapter 3. From now on, the EM centre is given by the average of $\left(X_{p 2+p 4}, Y_{p 1+p 3}\right)$ with a precision as the standard deviation. The error estimation, $\xi_{u}$, could be calculated as the difference between the two centres found using asymmetry measurements $\left\{A M_{1}, \ldots, A M_{4}\right\}$, similar to the theoretical accuracy estimated via simulations.

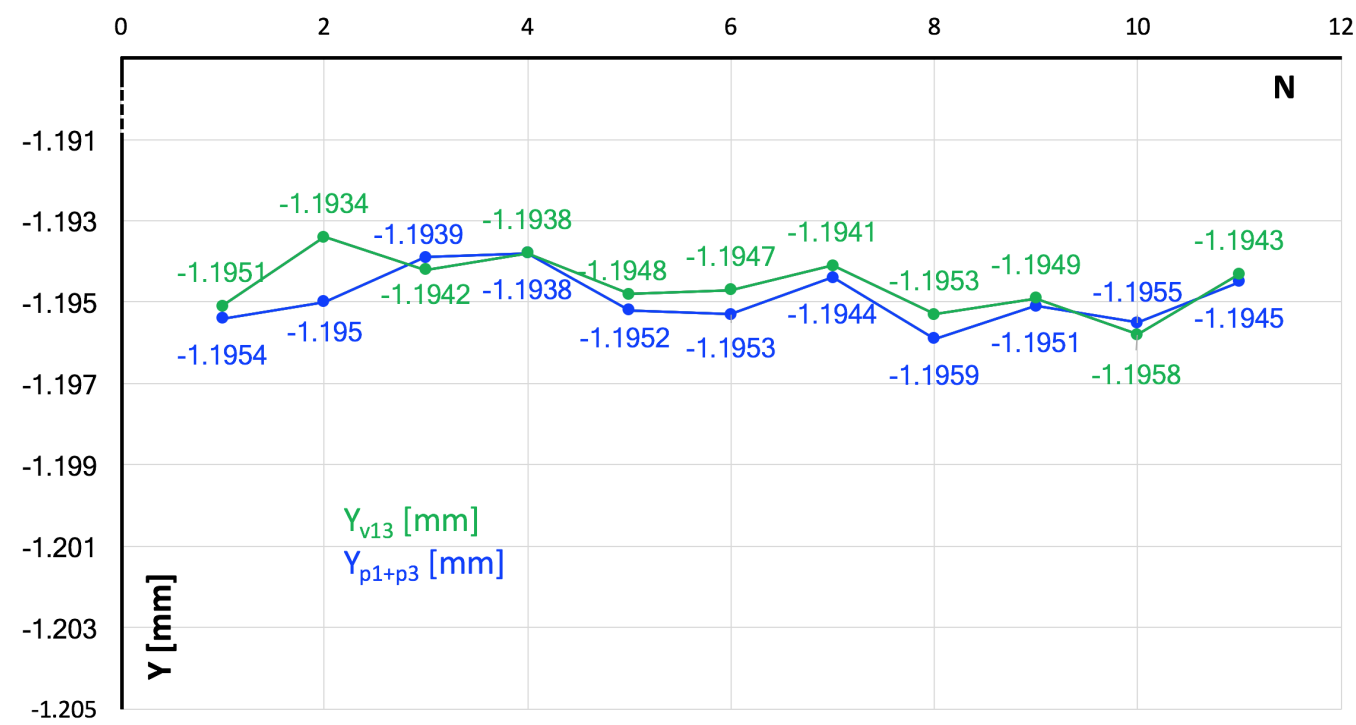

(a)

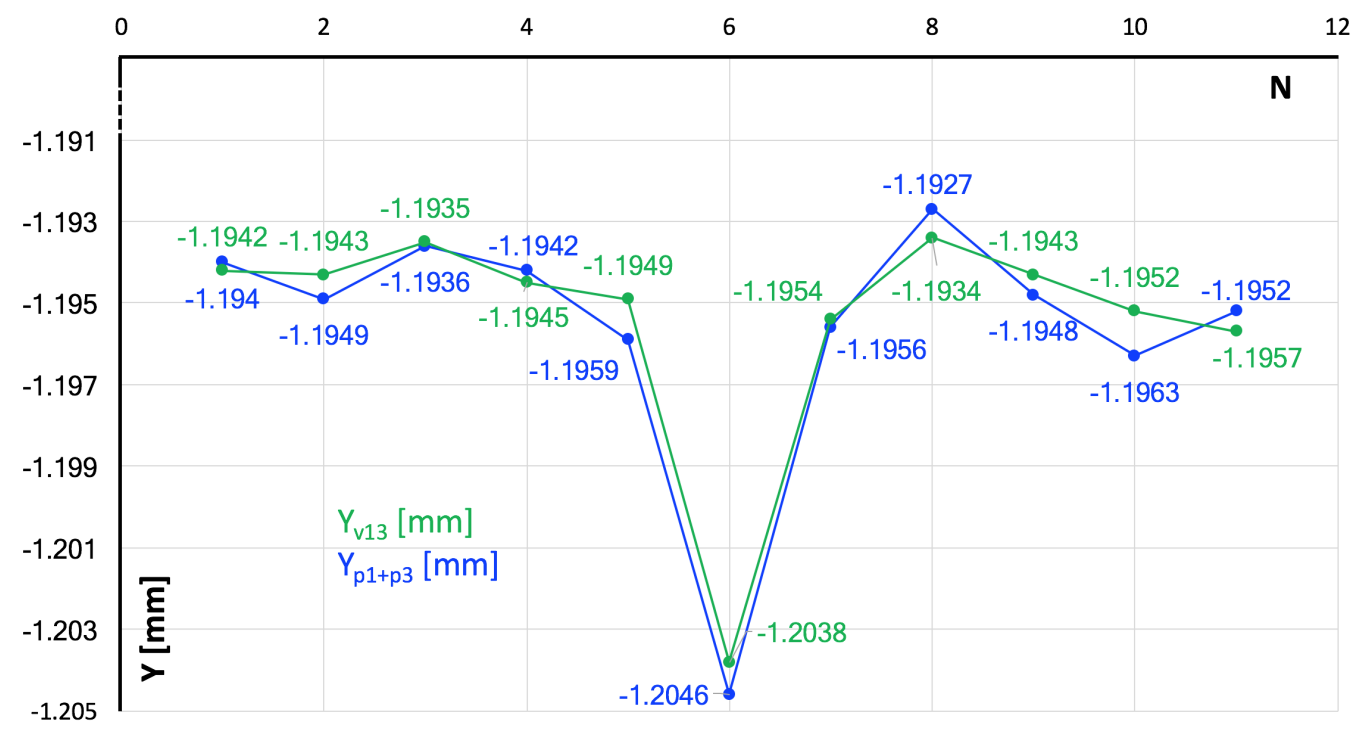

(b)

Figure 4.13: Convergence of the vertical coordinate of the EM centre when using arithmetic combination of $\left\{A M_{1}, \ldots, A M_{4}\right\}$ (a) against virtual port measurements (b). 


\section{Analysis of the precision and repeatability of the EM centre measurement.}

We performed several experimental tests in order to determine the precision and repeatability of the EM centre measurement with a fully calibrated test bench and optimised algorithm.

As a first experiment, we performed 64 consecutive measurements of the EM centre to determine the measurement precision. In Figure 4.14 (a) and (b), we present the standard deviation of the measurements in $X$ and $Y$, respectively. A precision of $0.63 \mu \mathrm{m}$ is obtained in the horizontal plane and $0.74 \mu \mathrm{m}$ in the vertical plane, both calculated at one sigma. These are very satisfactory results taking into account the cumulative positioning error of the stages. We found an error $\xi_{u}$ less than $\pm 8.5 \mu \mathrm{m}$, being very close to the accuracy limit of $\pm 7.5 \mu \mathrm{m}$ obtained with simulations. So, an evident improvement is obtained with respect to the previous test bench configurations.

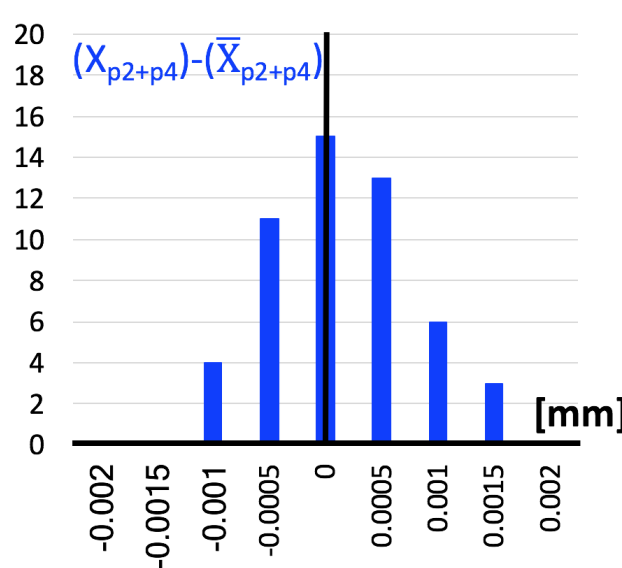

(a)

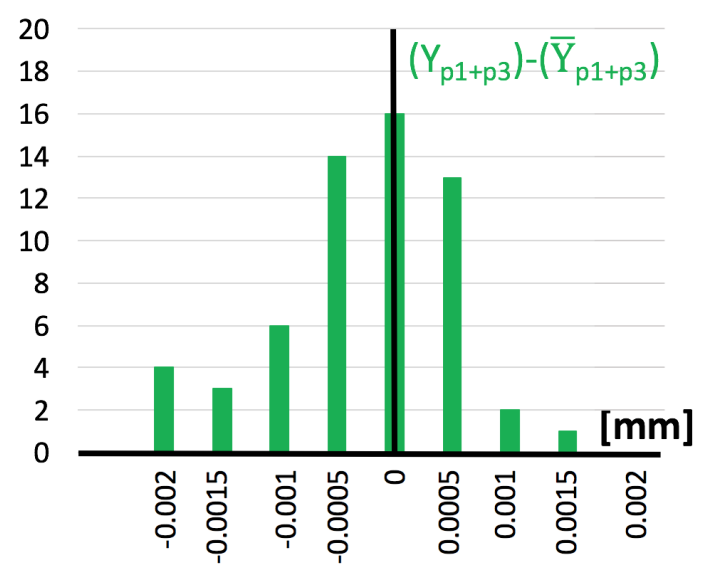

(b)

Figure 4.14: Precision of the EM centre in $X$ (a) and $Y$ (b) with tapered transitions, a calibrated test bench and optimised algorithm.

As a second experiment, the EM centre was measured 12 times in 8 different days following the usual procedure. The conditions of the measurements were changed in order to characterise the repeatability of the EM centre measurement. The results can be seen in Figure 4.15. We proceeded with the installation of the new wire in 4 different days, as indicated with the red circles. A fluctuation is observed between the measurements in days 3, 4 and 5, probably due to temperature rise. Repeatability is, in this case, better than $5 \mu \mathrm{m}$. Under the same conditions of temperature, wire tension and VNA calibration, repeatability was better than $0.5 \mu \mathrm{m}$. Conditions were lost when we changed the test configuration, for instance, installing a new wire.

The correlation between the two centres found with $\left\{A M_{1}, \ldots, U 4\right\}$ were also analysed. In Figure 4.16 (a), we plot $X_{p 2}$ with respect to $X_{p 4}$, and in (b), we plot $Y_{p 1}$ with respect to $Y_{p 3}$. A correlation is observed between the two centres in both planes, and is achieved thanks to the inclusion of the wire-positioning system and corresponding calibration. This result is an indication that noise was not the origin of the error $\xi_{u}$. 
Chapter 4: Experimental measurements of the EM axes of the TD24 and analysis using the perturbative method

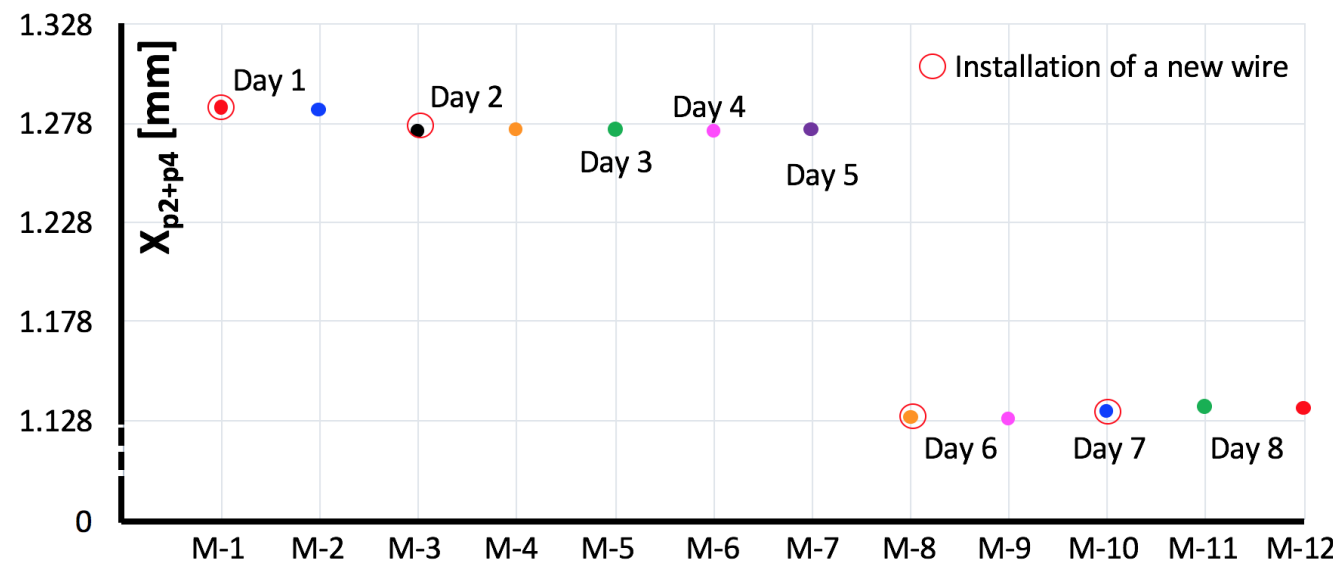

(a)

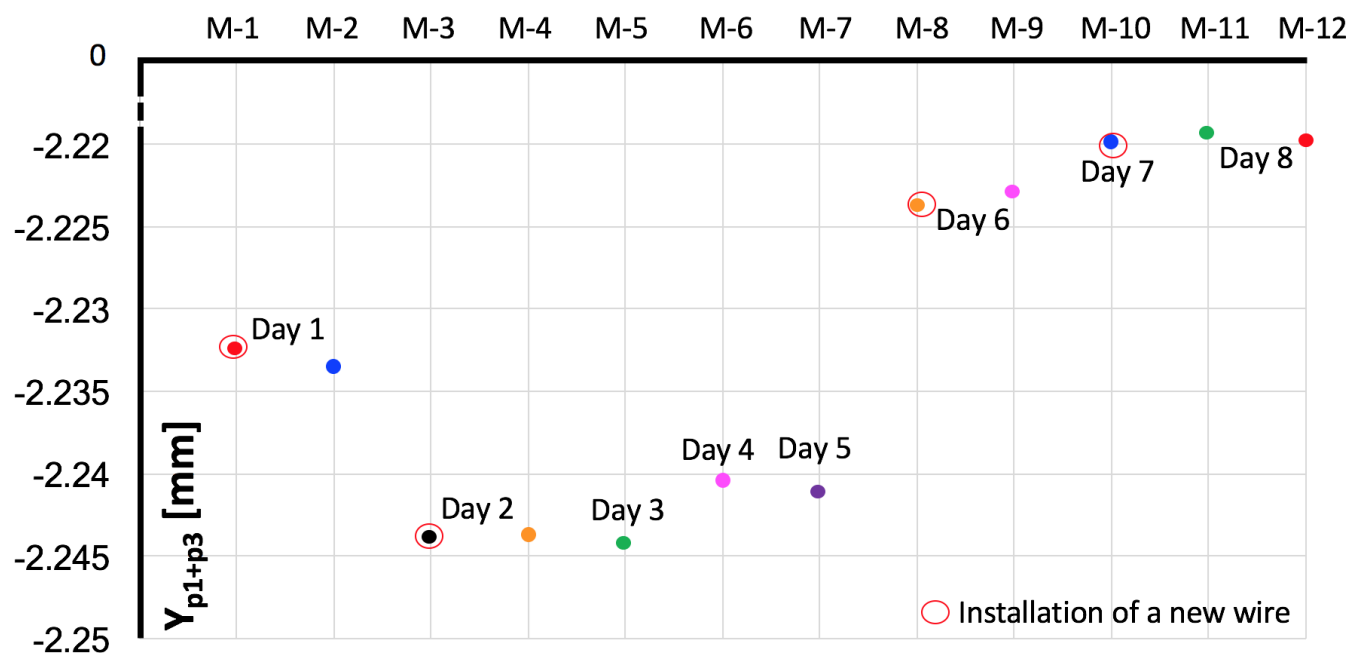

(b)

Figure 4.15: Repeatability measurements of the EM centre from 8 different days. The red circles indicates the installation of a new wire. (a) EM centre measured in $X$ with respect to the measurement number $M$. (b) EM centre measured in $Y$ with respect to $M$.

In this section, we conclude that the inclusion of the wire-positioning system, the fully calibration performance of the test bench and the optimised algorithm, improved precision and error of the EM centre measurement at the reference frequency. The EM centre was measured with a precision of $0.63 \mu \mathrm{m}$ in the horizontal plane and $0.74 \mu \mathrm{m}$ in the vertical plane. The estimation of the error in the position of the EM centre was $\xi_{u} \leq \pm 8.5 \mu \mathrm{m}$, only $1 \mu \mathrm{m}$ far from the theoretical accuracy limit. A repeatability of $0.5 \mu \mathrm{m}$ was obtained when performing the measurement of the EM centre under the same conditions of temperature, wire tension and calibrations; otherwise, it is $5 \mu \mathrm{m}$. These results are still well inside the required accuracy. 


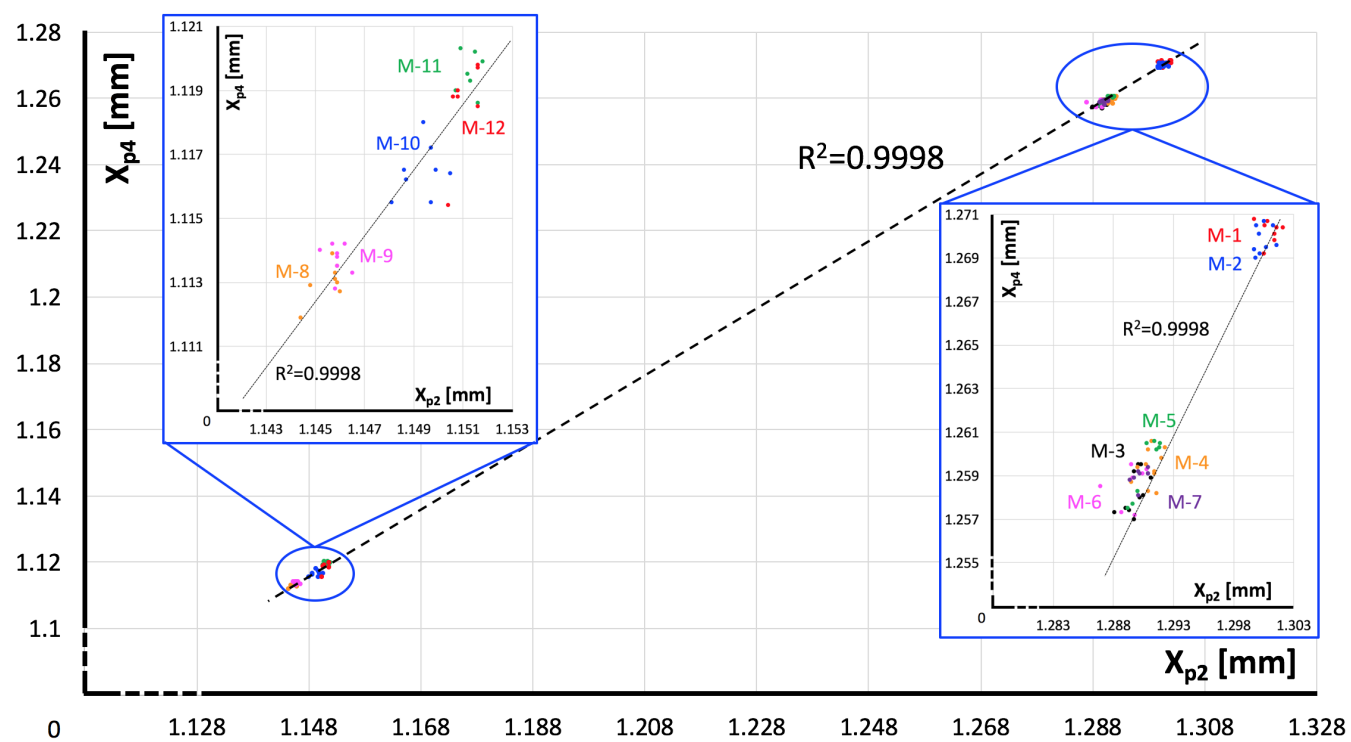

(a)

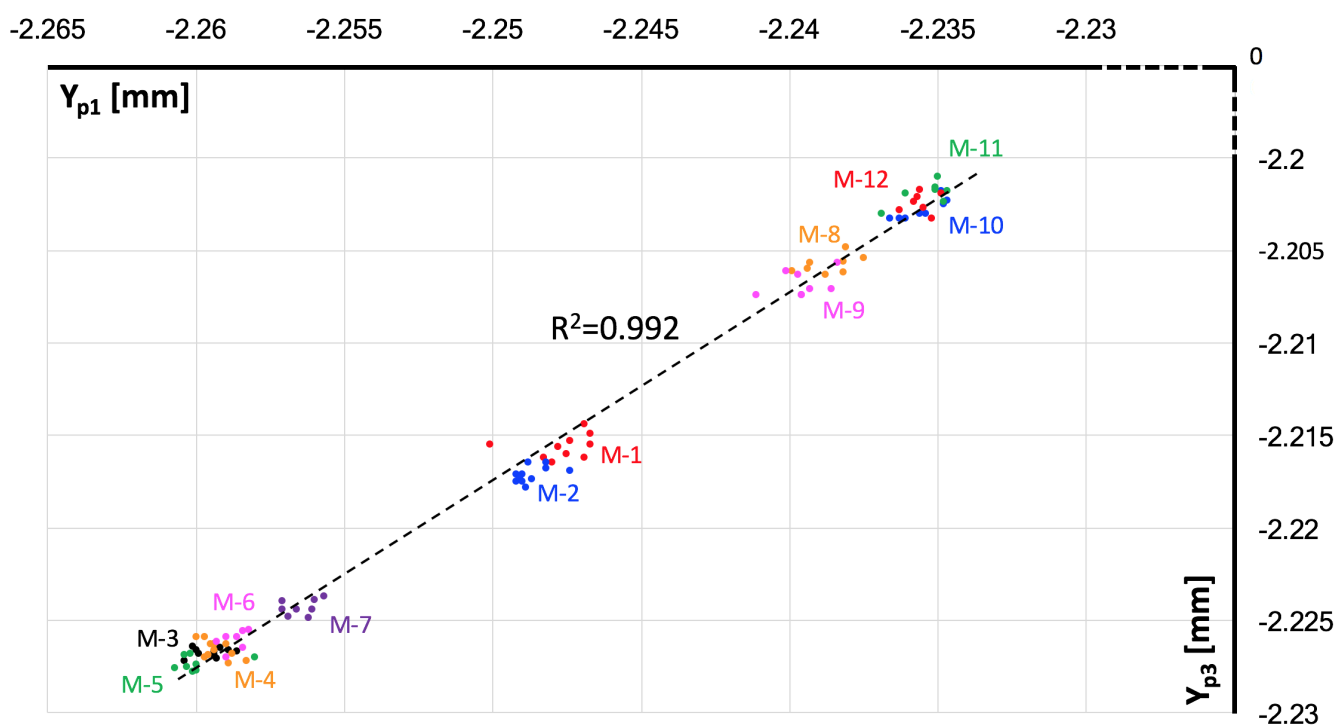

(b)

Figure 4.16: Correlation between the EM centre found for 8 different days. (a) Correlation between $X_{p 2}$ and $X_{p 4}$. (b) Correlation between $Y_{p 1}$ and $Y_{p 3}$. 


\subsubsection{Repeatability analysis of the perturbative method with metrology mea- surements}

Position measurements were performed in the context of the CMM at CERN, to crosscheck the repeatability of the EM centre measurement previously found with the perturbative method. For this purpose, the test bench was moved to the CMM, as shown in Figure 4.17 (a). 18 fixed spheres or targets were distributed around the test bench, as shown in Figure 4.17 (b), whose positions were measured by the sensor of the CMM. The absolute wire position was determined by 3 fiducials located in each wire positioning system. This reference was supported by a plane formed by 4 spheres placed on the frame of the test-bench. These 10 spheres formed the reference system of the bench. The perturbative method was applied to measure the EM centre of the TD24. The AS was moved and fixed to the computed EM centre, and the CMM sensor measured up to 8 fiducials glued on the structure. The 8 targets on the structure formed a second reference system that could be related to the reference system formed by the wire and the frame. Thus, the relative position of the AS with respect to the absolute wire position was obtained.

This process was repeated five times. We proceeded with the installation of a new wire at measurements 1, 3 and 4, changing the wire tension. The EM centre measured by the VNA (blue dots) and by the CMM (green dots) are shown in Figure 4.18. We had the problem that the fiducials of the wire-positioning system on top of the frame, fell during measurement 3 with the CMM. Therefore, the measurement was lost and is missing in the plot. Observing the figure, we could say that the relative movement of the CMM agree with the RF measurements. We also faced the problem that when the CMM performs a measurement, it moves the marble to position the target in such a way that the sensor accesses easily. When it happened, the heavy and hard cables that connected the stages to the controller, moved the stages. This problem was automatically solved by the controller, which positioned the stages every time it sensed a shift with respect to the given fixed position. We think that the difference between the metrology and the RF measurements is justified by this fact. However, the difference is so small (from $0.5 \mu \mathrm{m}$ to $2 \mu \mathrm{m}$ ) that we could rely in the positioning of the stages and in the perturbative method to measure the EM axes of AS. 


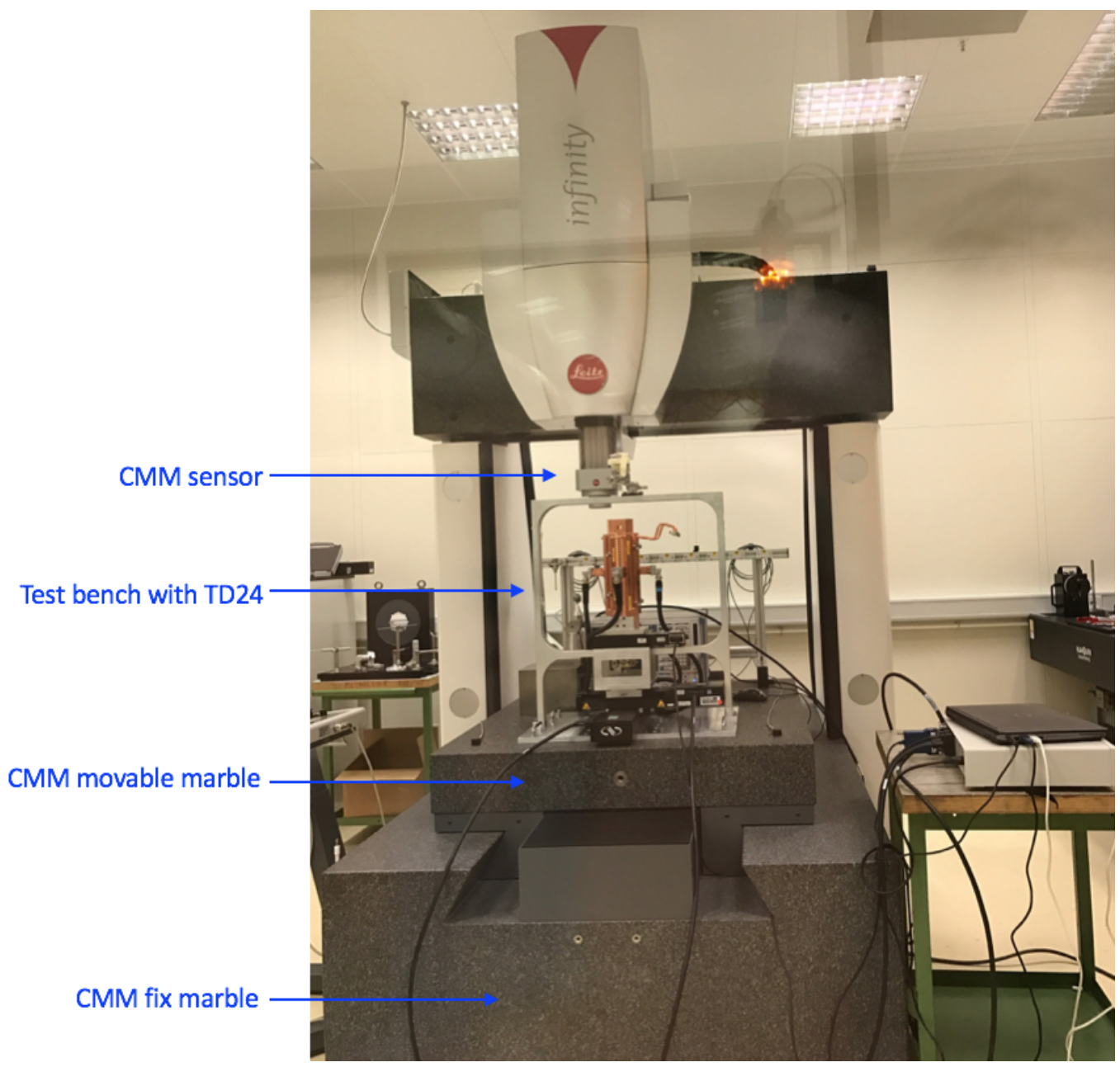

(a)

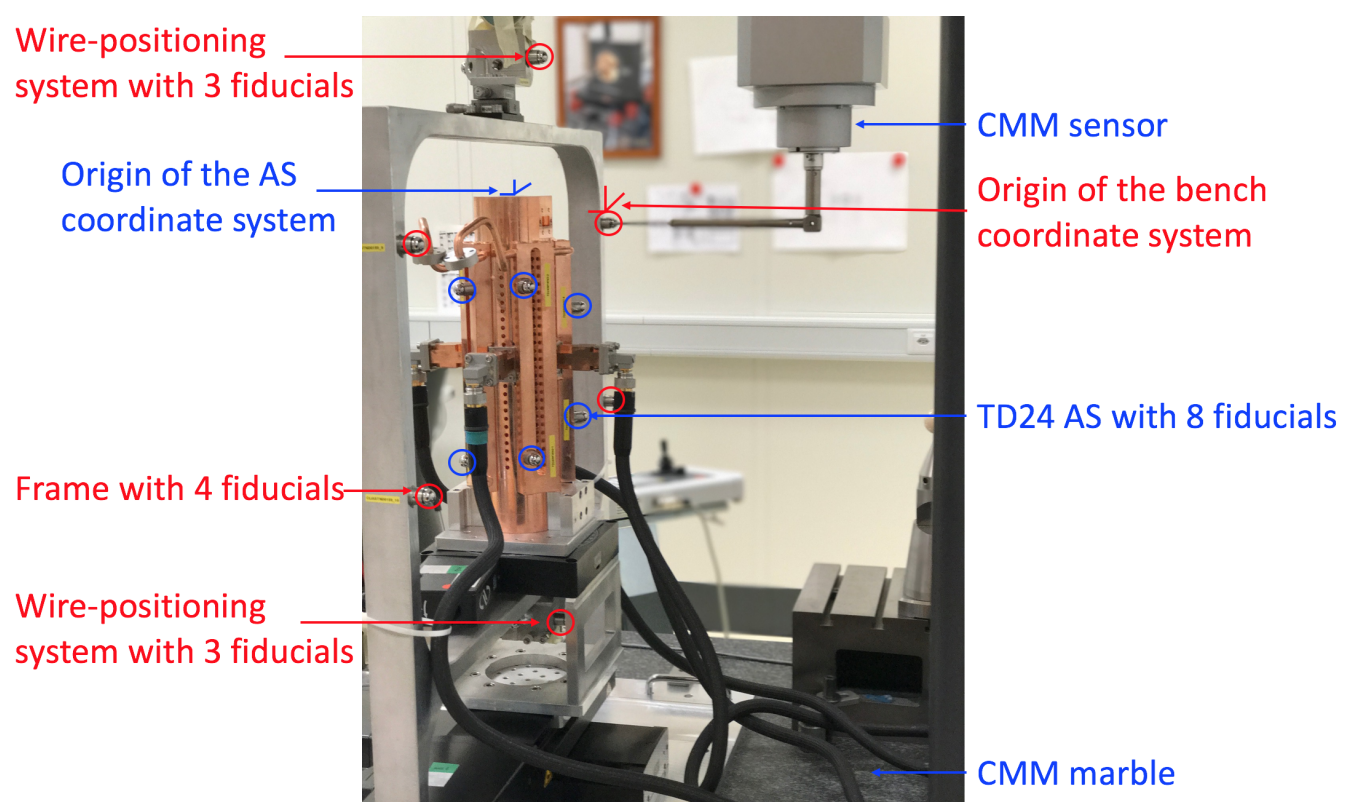

(b)

Figure 4.17: (a) General view of the test bench with TD24 AS mounted on the marble table of the Leitz Infinity. (b) Detailed view of the fiducials distribution glued in the test bench and in the TD24. Red circles indicate fiducials belonging to the reference system of the bench and blue circles, to the coordinate system of the AS. 
Chapter 4: Experimental measurements of the EM axes of the TD24 and analysis using the perturbative method

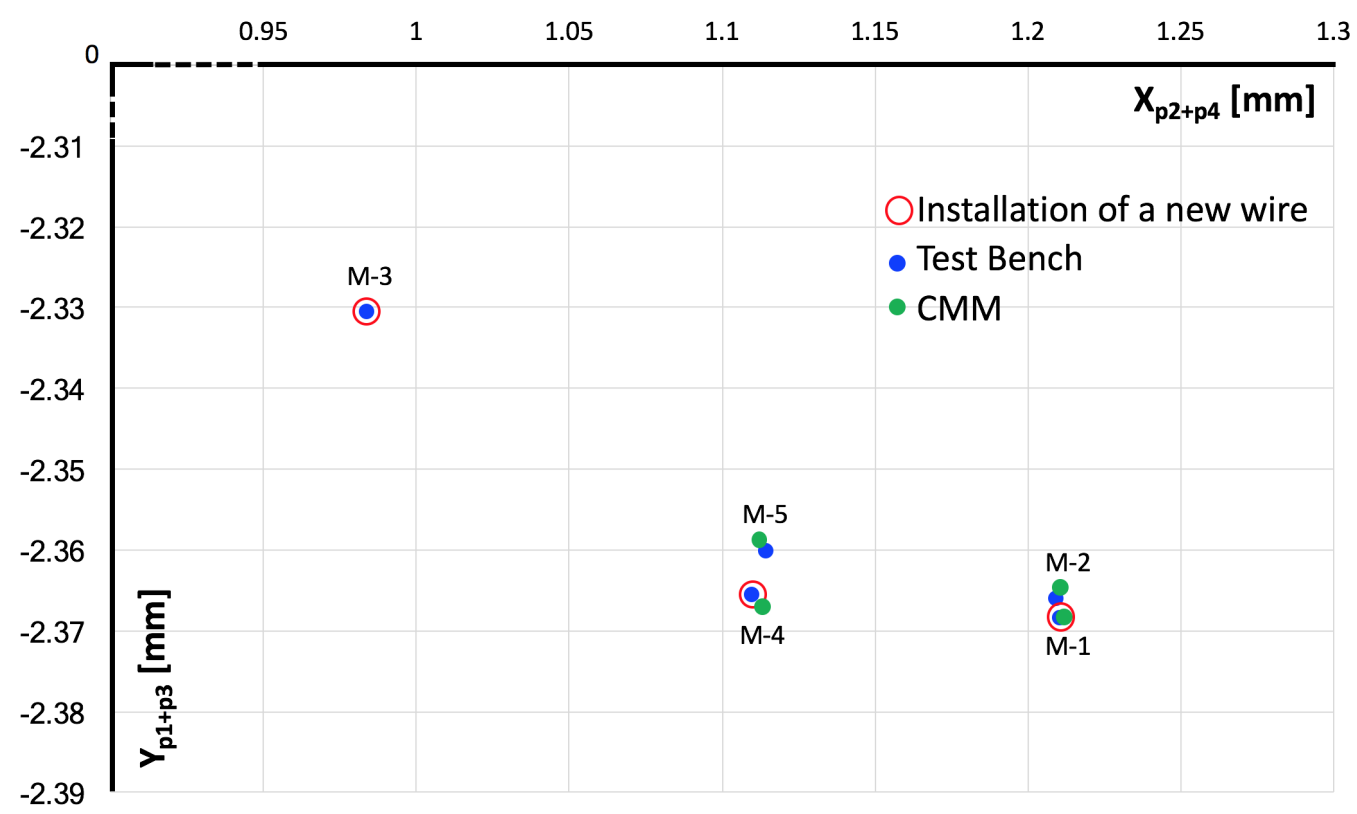

Figure 4.18: Measured position of the EM centre by the test bench (blue dots) and by the CMM (green dots) in $X$ and $Y$.

\subsection{Experimental identification of possible sources of errors}

In this section, we aim to analyse possible sources of errors present in the EM centre measurement. In particular, we will perform several experiments to determine how it is influenced by the longitudinal axis rotation, the coupling between the tapered transitions and the middle cell and temperature.

\subsubsection{Impact of rotating the TD24 around the longitudinal axis in the mea- surement of the EM centre}

We rotate the AS around its longitudinal $Z$ axis (or $\varphi$ ) to study the origin of the precision and error of the EM centre measurement. Furthermore, we analysed the possibility of an internal asymmetry in the middle cell and taper transitions. The experimental strategy was the following one: we first obtained the EM centre when $\varphi=0^{\circ}$ (or home position); second, we disconnected the VNA ports of the VNA, rotated the AS to $\varphi=90^{\circ}$ and connected back; third, we measured the EM centre with the new configuration and the results were compared.

The EM centre found with $\varphi=0^{\circ}$ was $(X, Y)=(1.6183,-2.4304) \mathrm{mm}$, and with $\varphi=90^{\circ}$ it was found at $(X, Y)=(1.8846,-2.4160) \mathrm{mm}$. So, the fact of touching the structure provoked a difference in the positioning of $266.3 \mu \mathrm{m}$ in the $X$ axis and $14.4 \mu \mathrm{m}$ in $Y$. The precisions are plotted in Figure 4.19 with respect to the mean value obtained for the centre. The green colours represent the non-rotated case, while the blue ones correspond to the rotated situation. From the plots, we could infer that both cases present the same standard deviation. The error obtained when $\varphi=90^{\circ}$ was $\xi_{u} \approx \pm 8 \mu \mathrm{m}$. Comparing with the previous 
situation, it seems that the stage is more stable at the home position, so we should avoid using the rotation stage at another position different from home.

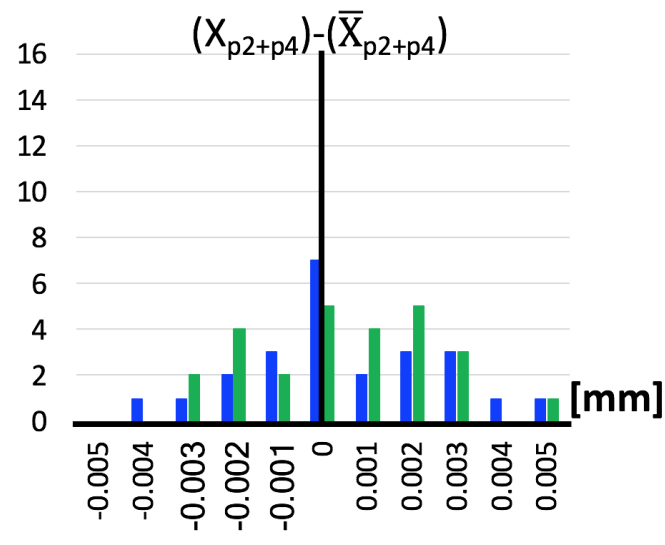

(a)

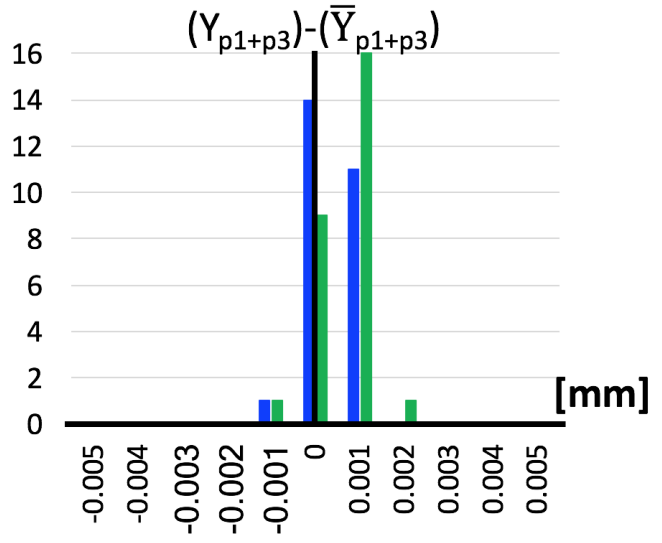

(b)

Figure 4.19: Precision of the EM centre measured in $X$ (a) and $Y$ (b), where the green bars represent the AS at $\varphi=0^{\circ}$ and the blue bars at $\varphi=90^{\circ}$.

The parameters of the last regression lines obtained in both measurements of the EM centre are compared to determine a possible asymmetry coming from the middle cell and the tapers. The corresponding asymmetry measurements, $A M$, in $Y$ when $\varphi=0^{\circ}$, can be seen in Figure 4.20 (a). The same is represented in both axes $X$ (b) and $Y$ (c) when $\varphi=90^{\circ}$. A few observations are done. First, the EM centre found in both cases were very similar. Second, there is an inversion of slope in the measurement given that both axis are rotated and the algorithm has not been adapted to this new situation. And third, the results in the $Y$ axis obtained when $\varphi=0^{\circ}$ are similar to the results in $X$ when $\varphi=90^{\circ}$. We expect that the same situation occurs in the other axis, but we can not verify this fact without the result for the $X$ axis when $\varphi=0^{\circ}$. Indeed, in (b) we observe that $\left|S_{32}\right|-\left|S_{12}\right|$ and $\left|S_{34}\right|-\left|S_{14}\right|$ with respect to the position of the linear stage in the horizontal plane can be approximated as two parallel lines. However, this trend is not followed when measuring $\left|S_{41}\right|-\left|S_{21}\right|$ and $\left|S_{43}\right|-\left|S_{23}\right|$ with respect to the linear stage in (a) and (c). These results are an indication that the eventual random errors do not come from the acquisition chain or the wire tilt, but from the cell geometry of the TD24 and potential asymmetry. Comparing the slopes of the regression lines in Figure 4.20 with respect to the simulation results, they coincide that there is a wire tilt below $0.5^{\circ}$. 
Chapter 4: Experimental measurements of the EM axes of the TD24 and analysis using the perturbative method

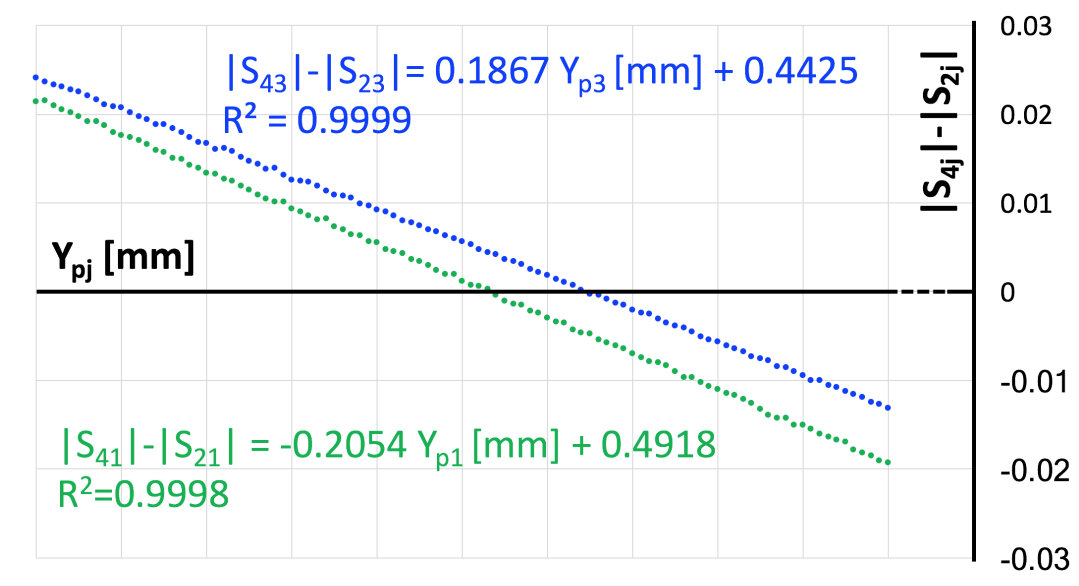

$\begin{array}{lllllllllll}-2.5 & -2.48 & -2.46 & -2.44 & -2.42 & -2.4 & -2.38 & -2.36 & -2.34 & -2.32 & -2.3\end{array}$

(a)

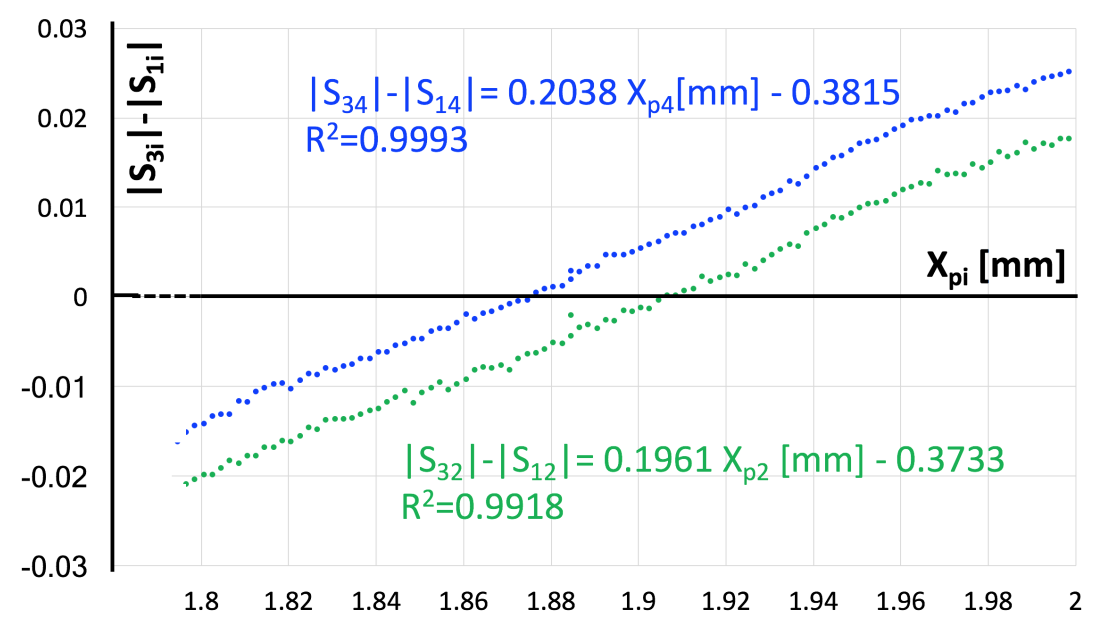

(b)

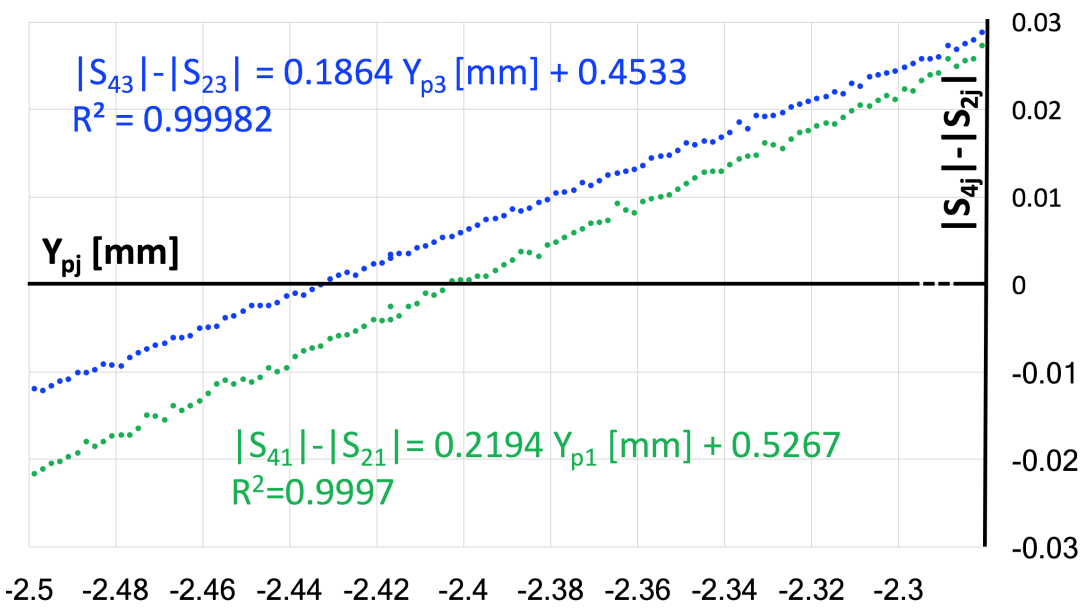

(c)

Figure 4.20: Asymmetry measurements in (a) $Y$ when $\varphi=0^{\circ}$. (b) $X$ when $\varphi=90^{\circ}$. (c) $Y$ when $\varphi=90^{\circ}$. 


\subsubsection{Impact of the coupling between the tapered transitions and the middle cell in the measurement of the EM centre}

We analysed the effect of the coupling between the tapered transitions and the middle cell in the measurement of the EM centre. This study was performed using different VNA port connections and configuration of tapers.

The experimental procedure consisted of four steps. First, the EM centre was found with the usual configuration of ports. Second, the process was repeated with tapers 2 and 4 switched with each other. Third, the EM centre was found switching tapers 1 and 3 , while $\mathrm{p} 2$ and $\mathrm{p} 4$ remained as the previous configuration. And finally, we disconnected the tapered transitions at ports 1,2 and 3 keeping the configuration in step 3 . They were connected again and the impact in the measurement of the centre was analysed.

The results concerning position and precision of the EM centre $(\mathrm{X}, \mathrm{Y})$ measured in each experiment are shown in Table 4.1. From experiment 1 to 2, we observe that the centre has moved $10.8 \mu \mathrm{m}$ in $X$ and $215.1 \mu \mathrm{m}$ in $Y$ and has a precision very similar in both cases. This result shows that the position measured as the EM centre is affected in the coordinate where the symmetry of the cell has been modified, being in this case $Y$. From the results of experiments 2 and 3, we infer the same result as before. In this case, the position of the centre was more affected in the $X$ axis by $54 \mu \mathrm{m}$, while it was only $1.5 \mu \mathrm{m}$ in $Y$. Note also that the symmetry concerning precision of the measured centre is recovered in both axes. Disconnecting and connecting again the tapers in experiment 4 , we measured a shift in the EM centre of $1.3 \mu \mathrm{m}$ in $X$ and $4.7 \mu \mathrm{m}$ in $Y$ with the same precision as in experiment 3 .

In view of these results we could conclude that the coupling between the tapered transitions and the middle cell could modify the geometry of the cell and, therefore could affect the measurement of the position of the EM centre by a few micrometers and also its precision by less than half a micron.

\begin{tabular}{|c|c|c|}
\hline Experiment number & EM centre $\left(X_{p 2+p 4}, Y_{p 1+p 3}\right)[\mathbf{m m}]$ & Precision $(X, Y)[\mu \mathrm{m}]$ \\
\hline 1 & $(0.7139,-2.3280)$ & $(0.30,0.65)$ \\
\hline 2 & $(0.7031,-2.5431)$ & $(0.28,0.67)$ \\
\hline 3 & $(0.7571,-2.5446)$ & $(0.26,0.28)$ \\
\hline 4 & $(0.7584,-2.5399)$ & $(0.26,0.28)$ \\
\hline
\end{tabular}

Table 4.1: Position of the EM centre in $X$ and $Y$ and precision.

\subsubsection{Influence of temperature in the measurement of the EM axes}

In view of improving the errors in the measurement of the EM centre and given the fact that we could control temperature, we characterised its impact in the position of the EM centre.

At each $S$ parameter measurement, the temperature data was stored, therefore, in Figure 4.21 we represent the progression of the EM centre in the vertical axis with respect 
to temperature. It can be observed that each hour, temperature raised up to $0.1^{\circ}$ and that it exists a correlation between the EM centre with the temperature change. In particular, we identified a drift on the EM centre detection of around $0.5 \mu \mathrm{m}$ caused by each temperature rise of around $0.1^{\circ}$. As already mentioned, the objective is to obtain the position of the EM centre with a precision from around 1 to $2 \mu \mathrm{m}$, so this value fixes the maximum temperature drift that we could afford.

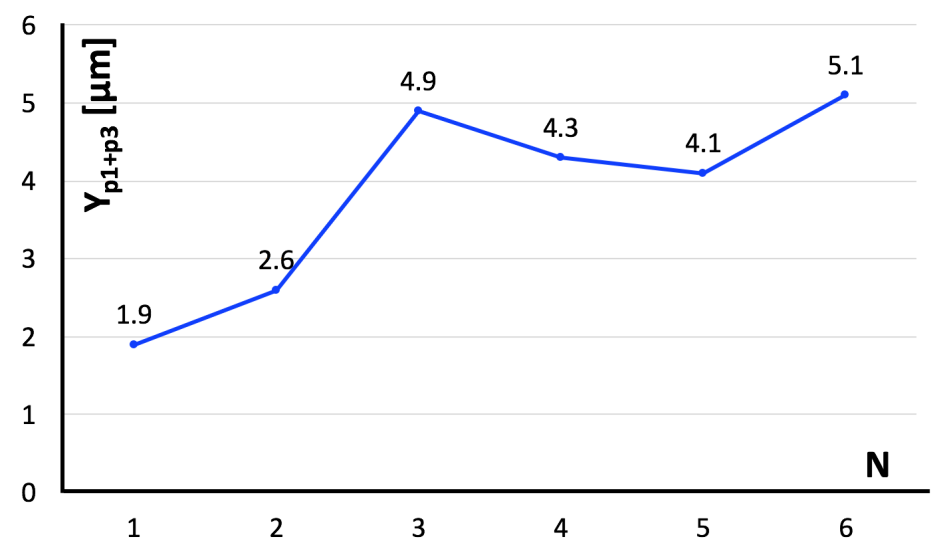

(a)

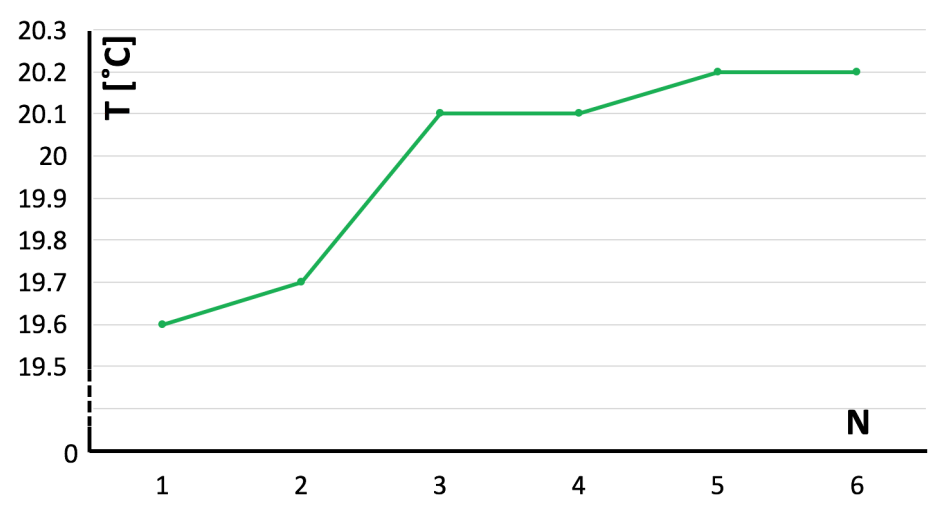

(b)

Figure 4.21: (a) Position of the measured EM centre in $Y$ with respect to the iteration number, $N$, and drift caused by temperature, $T$, rise. (b) Temperature, $T$, evolution during the EM centre measurement in $Y$.

From all these results, we could conclude that we have been able to measure the EM axis of the TD24 with the perturbative method using the test bench that we have developed for this purpose. Precisions of $0.63 \mu \mathrm{m}$ and $0.74 \mu \mathrm{m}$ are obtained in the horizontal and vertical planes, respectively, at a controlled temperature. The error estimation calculated as the difference between asymmetry measurements was $\xi_{u} \leq \pm \mathbf{8 . 5} \mu \mathbf{m}$. Under the same conditions of temperature, wire tension and calibration of the VNA, repeatability was better than $0.5 \mu \mathrm{m}$. These results are under the precision requirements from 1 to $2 \mu \mathbf{m}$. 


\section{Chapter 5}

\section{Summary and conclusion}

The innovative doctoral programme where this $\mathrm{PhD}$ thesis has been held, the PACMAN project, has boosted the precision and accuracy of the alignment of the next generation of accelerator components, namely quadrupole magnets, beam position monitors (BPM) and accelerating structures (AS). An alternative solution has been proposed to integrate all the alignment steps and a large number of technologies at the same time and location. In this way, the alignment strategy involves the determination of the EM axes of components with respect to alignment targets, or fiducialisation, and the initial alignment of components on a common support assembly. The methods and tools developed within this multidisciplinary project have been validated in individual test benches using the CLIC accelerator components. The EM axes have been determined and referenced in the coordinate frame of the common support assembly within the target accuracy below $10 \mu \mathrm{m}$. This is a first attempt to combine fiducialisation and transverse alignment of different types of accelerator components in the metre range, simultaneously on a common support within micrometric accuracy. The scientific accomplishments of PACMAN, cover the smallest high-precision rotating PCB coil for small magnet aperture ever manufactured, with a calibration error of $22 \mu \mathbf{m}$ in the radial coordinates and $\mathbf{6 2} \mu \mathbf{m}$ in the vertical coordinates; the most accurate portable system for large scale absolute measurements, with an uncertainty of $\mathbf{4} \mu \mathbf{m}$ and an accuracy on the wire positioning and the wire orientation of $\mathbf{2 0} \mu \mathrm{m}$ and $\mathbf{4 0} \mu \mathbf{m} / \mathbf{m}$, respectively; the first seismic sensor integrating three different transducers compatible with an electromagnetic environment, with a bandwidth from 0.1 to $200 \mathrm{~Hz}$ and a resolution of $\mathbf{0 . 1} \mathbf{~ n m ~ r m s ~ a t ~} 1 \mathbf{~ H z}$; the smallest achieved BPM resolution using a bench wire instead of a beam, with a value of $\mathbf{1 2} \mathbf{~ n m}$; the first four-degrees of freedom nano-positioning system with motion control of $\mathbf{5 0} \mathbf{~ n m}$, integrating high loads, magnetic field and vibration stabilisation of $1.5 \mathbf{~ n m ~ r m s ~ a t ~} 1 \mathbf{~ H z}$; and the most accurate absolute fiducialisation process of quadrupole, BPM and AS ever achieved with a repeatability of $\mathbf{1} \mu \mathbf{m}$.

In this thesis, we have developed a new non-destructive intra-cavity technique to determine the EM axes of ASs by means of a stretched wire in a laboratory environment, based in the Slater perturbation theorem and named here as the perturbative method. This method exploits the hypothesis that the originally excited EM fields in one cell of the AS could be perturbed by the presence of a stretched conductor wire along the AS, by changing transmitted power signals between ports. The change in the EM fields could be 
monitored in the cell at any wire position in the Scattering parameter form, using a simple vector network analyzer (VNA). The perturbation preserves the symmetry in the cell and gives the position of the EM centre at the minimum. The perturbative method was applied in the heavily damped CLIC TD24 AS, whose complex geometry and small iris mean aperture of $\mathbf{5 . 5} \mathbf{~ m m}$ makes impossible the applicability of the existing techniques.

The feasibility of the perturbative method was assessed by extensive EM field simulations in HFSS, considering the special characteristics of the TD24 that is only internally accessible through wakefield monitors (WFM) in the middle cell. As a result, we could conclude that the EM centre could be measured with $\mathbf{1} \mu \mathbf{m}$ resolution, and a theoretical accuracy limit of $\pm 7.5 \mu \mathrm{m}$. However, in order to optimise time and resources, a maximum resolution of $\mathbf{2 0} \mu \mathbf{m}$ gives the same result for the centre with a deviation below $\mathbf{1} \mu \mathbf{m}$, when moving the wire in a minimum travel range of $\mathbf{1 0 0} \mu \mathrm{m}$ around the position of the EM centre. Hardware imperfections were simulated to identify and characterise possible sources of errors, for instance, wire offset with respect to the transverse plane, wire tilt in the longitudinal axis, and coupling between the transitions connecting the middle cell of the TD24 to the VNA. We applied these results in the conceptual design of the measurement algorithm, and in the establishment of technical specifications of an experimental laboratory set-up.

A dedicated test bench was designed, manufactured and calibrated. Given the length of the TD24, $\mathbf{3 4 1 . 1} \mathbf{~ m m}$, the set-up configuration was such that the AS was installed vertically on top of two orthogonal linear stages, allowing the movement of the AS in two degrees of freedom, the easy-up introduction of the wire with gravity and the elimination of its sag. The bench was assembled in the accurate environment of a coordinate measuring machine $(\mathrm{CMM})$ striving to error minimisation, with a position uncertainty of around $\mathbf{0 . 3} \mu \mathbf{m}$. Furthermore, we integrated the algorithm in a LabVIEW user platform, allowing the automatisation of the process that gives the position of the stages where the perturbation measured by the VNA is minimised.

We performed a full experimental test to verify the hypothesis of the perturbative method, and we found a good agreement with the EM field simulation results. The proof-ofprinciple was performed using the WFM signals, but the Scattering parameters showed different sensitivities in each plane. After the first iterations of the algorithm, the EM centre, as measured by each port, converged very rapidly into a unique value. However, we found a different value for the centre of around $\mathbf{1 8} \mu \mathrm{m}$ in the $X$ axis, and $5 \mu \mathbf{m}$ in the $Y$ axis. In order to recover the symmetry in the middle cell, the WFM, originally designed to extract HOM power signals and not input RF signals, was replaced by tapered transition waveguides. The EM centre was measured with symmetric sensitivity, but an asymmetric difference between the measured centres at each plane still existed. Precisions between 1 to $\mathbf{5} \mu \mathbf{m}$ were found in $X$ and $Y$, respectively, with an error of around $\pm \mathbf{1 0} \mu \mathbf{m}$. To solve this, we arithmetically recombined the measurements from both ports, performed the fully calibration of the test bench, and characterised the main sources of errors. The EM centre was measured again, and precisions of $0.63 \mu \mathrm{m}$ in the horizontal plane and $0.74 \mu \mathrm{m}$ in the vertical plane were obtained, with an error below $\pm \mathbf{8 . 5} \mu \mathbf{m}$ very close to the theoretical accuracy limit obtained via simulations. Under the same conditions of temperature, tension of the wire and calibration of the VNA, repeatability was better than $\mathbf{0 . 5} \mu \mathbf{m}$. When the experimental conditions were changed, for instance, with the installation of a new wire, 
repeatability was around $\mathbf{5} \mu \mathrm{m}$. These results were crosschecked in the CMM, whose agreement ranged from $0.5 \mu \mathrm{m}$ to $2 \mu \mathrm{m}$.

We have also analysed and laid down the future lines of research. In particular, we proposed the application of the perturbative method in all cells to measure the cell-to-cell misalignment. We suggest including this step before bonding to potentially improve the current assembly tolerances between cells. Furthermore, we studied the feasibility of increasing the number of VNA ports to measure simultaneously the EM centre in various cells along the AS. This could allow positioning the wire with respect to the average EM centre of the structure, leading to a possible wakefield minimisation. In the framework of PACMAN, we also tested the possibility to locate the EM axes in the TD24 parallel to the beam direction. However, given the length of the AS, a mechanical solution for the easy-up installation of the wire and for the minimisation of the wire sag needs to be carefully investigated.

From this work, we conclude that we can locate the EM axes of AS with the perturbative method, a new simple technique consisting on straightforward high-frequency VNA measurements in a relatively inexpensive laboratory test bench with a thin conductor stretched wire instead of a beam. Despite the simplicity of the method, it pushes position measurements to precisions below $\mathbf{1} \mu \mathbf{m}$ within heavily damped AS with difficult accessibility and small aperture irises of a few millimetres. Compared to other existing methods, it does not need complicated hardware and challenging mechanics contributing to measurement errors. The perturbative method can be implemented in all AS types and in all waveguide applications in general. In other words, the PACMAN developments provide very interesting perspectives for the initial alignment and fiducialisation of the CLIC components, that can also be extrapolated to other international projects as ILC and other domains such as aerospace and automotive industry. 


\section{Bibliography}

[1] The European Strategy for Particle Physics Update 2013, Cern-Council-S/106, May 2013.

[2] Alexander Wu Chao, Handbook of Accelerator Physics and Engineering, 2013.

[3] H. Mainaud Durand, Alignment, Linear Collider School Lecture Series "Collider Technology", Mt. Fuji, Japan, 2016.

[4] CLIC Conceptual Design Report (CDR), http://project-clic-cdr.web. cern.ch/project-clic-cdr/CDR_Volume1.pdf, 2012

[5] P.N. Burrows, L. Linssen, D. Shulte, E. Sicking, S. Stapnes, M.A. Thomson, Updated Baseline for a Staged Compact Linear Collider, Geneva, 2016.

[6] International Linear Collider Technical Design Report, https://www . linearcollider.org/ILC/Publications/Technical-Design-Report, 2013.

[7] D. Shulte, Beam Based Alignment in the new CLIC Main Linac, Particle Accelerator Conference, Vancouver, Canada, 2009.

[8] W. Wuensch, Normal conducting RF, Linear Collider School Lecture Series: "RF technology", Turkey, 2013.

[9] K. Artoos, M. Modena, H. Mainaud-Durand, CLIC Quadrupole Module Final Report, 2013.

[10] H. Mainaud Durand, CDR: feasibility demonstration of active pre-alignment, Geneva, Switzerland.

[11] H. Mainaud Durand, T. Touze, The active pre-alignment of the CLIC components, International Workshop on Accelerator Alignment, Stanford, USA, 2006.

[12] H. Mainaud Durand, P. Bestmann, A. Herty, A. Marin, V. Rude, oWPS versus cWPS, IWAA2012, Batavia, US, 2012.

[13] FOGALE nanotech website, http://www . fogale.fr/

[14] PACMAN website, https://pacman.web.cern.ch

[15] H. Mainaud-Durand, K. Artoos, M. Buzio, D. Caiazza, N. Catalan Lasheras, A. Cherif, I. Doytchinov, J-F Fuchs, A. Gaddi, N. Galindo Munoz, J-C. Gayde, S.W. Kamugasa, M. Modena, P. Novotny, S. Russenschuck, C. Sanz, G. Severino, D. 
Tshilumba, V. Vlachakis, M. Wendt, S. Zorzetti, Fiducialisation and initial alignment of CLIC component with micrometric accuracy, IWAA2016, Grenoble, France, 2016.

[16] Leitz Infinity Coordinate Measuring Machine, http://www.hexagonmi . com/products/coordinate-measuring-machines/bridge-cmms/ leitz-infinity

[17] H. Mainaud Durand, K. Artoos, M. Buzio, D. Caiazza, N. Catalan Lasheras, A. Cherif, I. Doytchinov, A. Faus-Golfe, J-F Fuchs, A. Gaddi, N. Galindo Munoz, J-C Gayde, S. W. Kamugasa, M. Modena, P. Novotny, S. Russenschuck, C. Sanz, G. Severino, D. Tshilumba, V. Vlachakis, S. Zorzetti, Main Achievements of the PACMAN Project for the Alignment at Micrometric Scale of Accelerator Components, IPAC2017, Copenhagen, Denmark, 2017.

[18] N. Chritin, H. Schmickler, L. Soby, A. Lunin, N. Solyak, M. Wendt, V. Yakovlev, A high resolution cavity BPM for the CLIC Test Facility, BIW10, Santa Fe, New Mexico, 2010.

[19] A. Grudiev, W. Wuensch, Design of the CLIC Main Linac accelerating structure for CLIC Conceptual Design Report, LINAC2010, Tsukuba, Japan, 2010.

[20] N. Catalan Lasheras, H. Mainaud-Durand, M. Modena, Measuring and aligning accelerator components to the nanometre scale, IPAC2014, Dresden, Germany, 2014.

[21] C. Sanz, A. Cherif, H. Mainaud Durand, P. Morantz, P. Shore, Characterisation and measurement to the sub-micron scale of a reference wire position, CIM2015, Paris, France, 2015.

[22] C. Sanz, Rotating sensor for new possibilities on Leitz Infinity coordinate measuring machine, EUSPEN2016, Nottingham, UK, 2016.

[23] C.Sanz, A. Cherif, H. Mainaud-Durand, J. Schneider, N. Steffens, P. Morantz, P. Shore, New potential for Leitz Infinity Coordinate Measuring Machine, EUSPEN2015, Leuven, Belgium, 2015.

[24] S. Kamugasa, J.C. Gayde, H. Mainaud Durand, Frequency Scanning Interferometry for CLIC component fiducialisation, IWAA 2016, Grenoble, France, 2016.

[25] S. Kamugasa, Spherical adapter for FSI fibre end to realise true absolute distance measurement with the Absolute Multiline, Technical Report, CERN EDMS 1611901.

[26] S. Kamugasa, High index retroreflector for the Absolute Multiline: reflectivity, repeatability and calibration, Technical Report, CERN EDMS 1611902.

[27] S. Kamugasa, Study of thermally induced drift whilst measuring with he absolute multiline, Technical Report, CERN EDMS 1611905.

[28] S. Kamugasa, Compatibility tests of absolute multiline network to targets on the Leitz Infinity CMM, Technical Report, CERN EDMS 1611906.

[29] B. Burki, S. Guillaume, P. Sorber, H. Oesch, DAEDALUS: A versatile usable digital clip-on measuring system for Total Stations, IPIN2010, Zurich, Switzerland, 2010. 
[30] S. Guillaume, B. Burki, S. Griffet, H. Mainaud Durand, QDaedalus: Augmentation of Total Stations by CCD Sensor for Automated Contactless High-Precision Metrology, FIG2012, Rome, Italy, 2012.

[31] V. Vlachakis, Wire detection algorithms of a CuBe wire, Technical Report, CERN EDMS 1611908.

[32] V. Vlachakis, Wire reconstruction algorithms of a CuBe wire, Technical Report, CERN EDMS 1611909.

[33] V. Vlachakis, Final configuration of micro-triangulation for the FAP, Technical Report, CERN EDMS 1611907.

[34] V. Vlachakis, Recent development of micro-triangulation for magnet fiducialization, IWAA 2016, Grenoble, France, 2016.

[35] A.B. Temnykh, Vibrating wire field-measuring technique, Nuclear Instruments and Methods in Physics Research, Section A: Accelerators, 390 Spectrometers, Detectors and Associated Equipment, 1997.

[36] M. Modena, A. Bartalesi, J. Garcia Perez, R. Leuxe, G. Perrin-Bonnet, C. Petrone, M. Struik, A. Vorozhtsov, Performance of the Main Beam quadrupole type 1 prototypes for CLIC, IEEE transactions on applied superconductivity, vol. 24, no. 3, 2014.

[37] D. Caiazza, Compatibility tests for the operation of the PACMAN stretched-wire system in the environment of a Coordinate Measuring Machine, Technical Report, CERN EDMS 1611911.

[38] D. Caiazza, P. Arpaia, C. Petrone, S. Russenchuck, Performance of the stretch and vibrating-wire techniques and correction of background fields in locating quadrupole magnetic axes, IMEKO2015, Prague, Czech Republic, 2015.

[39] D. Caiazza, P. Arpaia, C. Petrone, S. Russenchuck, Uncertainty analysis of a vibrating-wire system for magnetic axes localization, ICST2015, Auckland, New Zealand, 2015.

[40] D. Caiazza, Preliminary tests for the magnetic alignment of a CLIC main beam quadrupole in the frame of the PACMAN project, Technical Report, CERN EDMS 1611910.

[41] O. Dunkel, A rotating coild array in "mono-bloc" Printed Circuit technology for small scale harmonic measurements, IMMW17, La Mola, Spain, 2011.

[42] J. DiMarco, Application of PCB and FDB technologies to magnetic measurement probe development, IMMW18, Upton, USA, 2013.

[43] I. Doytchinov, Uncertainty budgeting for large scale components production and micro precision accelerators pre-alignment as required by CLIC project, IWAA2016, Grenoble, France, 2016.

[44] K. Summerhays, J.M. Blandwin, D. Campbell, R. Henke, Application to simulation software to coordinate measurement uncertainty evaluation, Measure 2, 40-52, 2007. 
[45] P. Novotny, What is the Best Displacement Transducer for a Seismic Sensor?, INERTIAL17.

[46] S. Janssens, Stabilisation and precision pointing of a quadrupole magnet in the Compact Linear Collider (CLIC), PhD thesis, CERN/NIKHEF, 2015.

[47] D. Tshilumba, Technical Review Report I, Technical Report, CERN EDMS 1609106.

[48] D. Tshilumba, PACMAN mid-term review presentation, Technical Report, CERN EDMS 1609105.

[49] D. Ziemianski, M. Guinchard, Experimental modal analysis of the CLIC type 1 module, Technical Report, CERN EDMS 1427645.

[50] S. Zorzetti, L. Fanucci, N. Galindo Munoz, M. Wendt, Design of the $15 \mathrm{GHz} B P M$ test bench for the CLIC test facility to perform precise stretched-wire RF measurements, Measurements Science and Technology, DOI: 10.1088/09570233/26/9/094005.

[51] S. Zorzetti, L. Fanucci, N. Galindo Munoz, M. Wendt, Stretched wire techniques and measurements for the alignment of a $15 \mathrm{GHz} R F$ BPM for CLIC, IBIC2015, Melbourne, Australia, 2015.

[52] S. Zorzetti, M. Wendt, L. Fanucci, Study of the electrical center of a resonant cavity Beam Position Monitor (RF-BPM) and its integration with the main quadrupole for alignment purposes, NAPAC16, Chicago, USA, 2016.

[53] S. Zorzetti, Radiofrequency characterization and alignment to the nanometre scale of a Beam Position Monitor for particle accelerators, $\mathrm{PhD}$ thesis, University of Pisa, Italy, 2016.

[54] S. Zorzetti, K. Artoos, F.N. Morel, P. Novotny, D. Tshilumba, M. Wendt, L. Fanucci, A wire-based methodology to analyse the nanometric resolution of an RF cavity BPM, IBIC2016, Barcelona, Spain, 2016.

[55] H. Mainaud Durand, K. Artoos, M. Buzio, D. Caiazza, N. Catalan Lasheras, A. Cherif, I. Doytchinov, J-F. Fuchs, A. Gaddi, N. Galindo Munoz, J-C Gayde, S. W. Kamugasa, M. Modena, P. Novotny, S. Russenschuck, C. Sanz, G. Severino, D. Tshilumba, V. Vlachakis, M. Wendt, S. Zorzetti, PACMAN project: a new solution for the high-accuracy alignment of accelerator components. IPAC2016, Busan, Korea, 2016.

[56] S. Kamugasa, V. Vlachakis, PACMAN study of FSI and micro-triangulation for the pre-alignment of CLIC, IWAA2014, Beijing, China, 2014.

[57] A. Grudiev, W. Wuensch, Design of an X-Band Accelerating Structure for the CLIC Main Linac, LINAC08, Victoria, Canada, 2008.

[58] A. Grudiev, RF design and parameters of 12 GHz TD24-vg1.8-disk, Technical Report, CERN EDMS 1070498.

[59] A. Grudiev, W. Wuensch, Design of the CLIC Main Linac Accelerating Structure for CLIC Conceptual Design Report, LINAC2010, Tsukuba, Japan, 2010. 
[60] Thomas P. Wangler, RF Linear Accelerators, 2008.

[61] R.M. Jones, Wakefield suppression in high gradient linacs for lepton linear colliders, Physical Review Special Topics - Accelerators and Beams, Vol 12, 104801, 2009.

[62] F. Peauger, W. Farabolini, P. Girardot, A. Andersson, G. Riddone, A. Samoshkin, A. Solodko, R. Zennaro, Wakefield Monitor Development for CLIC Accelerating Structure, LINAC2010, Tsukuba, Japan, 2010.

[63] M. Dehler, S. Atieh, D. Gudkov, S. Lebet, G. Riddone, J. Shi, A. Citterio, R. Zennaro, G. D Auria, Fabrication of the CERN/PSI/ST X-Band Accelerating Structures, IPAC2011, San Sebastian, Spain, 2012.

[64] F. Peauger, Progress on TD24 accelerating structures fabrication and wakefield monitor experiment preparation, CEA, Saclay, 2012.

[65] R. Montonen, I. Kassamakov, E. Haeggstrom, K. Osterberg, Calibration of Fourier domain short coherence interferometer for absolute distance measurements, Applied Optics, Vol. 54, No. 15, 2015.

[66] R. Montonen, I. Kassamakov, E. Haeggstrom, K. Osterberg, Quantifying height of ultraprecisely machined steps on oxygen-free electronic copper disc using Fourierdomain short coherence interferometry, Optical Engineering, Vol 55, 014103, 2016.

[67] M. Sands, J. Rees, A bench measurement of the energy loss of a stored beam to a cavity, SLAC-TN-05-051, 1974.

[68] M.J. Barnes, F. Caspers, T. Kroyer, E. Metral, F. Roncarolo, B. Salvant, Measurement of longitudinal and transverse impedance of kicker magnets using the coaxial wire method, PAC09, Vancouver, Canada, 2009.

[69] N. Baboi, R.M. Jones, G.B. Bowden, V. Dolgashev, S.G. Tantawi, J.W. Wang, Coaxial Wire Measurements in NLC Accelerating Structures, EPAC2002, Paris, France, 2002.

[70] C. E. Deibele, Analysis of ultra-relativistic charged particle beam and stretched wire measurement interactions with cylindrically symmetric structures, $\mathrm{PhD}$ thesis, University of Wisconsin, Madison, USA, 1996.

[71] R. M. Jones, Fundamentals of wakefields and collective effects: from physicalmathematical analysis to practical applications, Series Lecture, University of Manchester/ Cockcroft Institute, 2015.

[72] R. M. Jones. Linear Accelerators: Theory and Practical Applications, Series Lecture, University of Manchester/ Cockcroft Institute, 2017.

[73] N. Baboi, G. B. Bowden, R. M. Jones, S. G. Tantawi, J. R. Lewandowski. Transverse Impedance Bench Measurements in NLC/JLC Accelerating Structures, PAC03, Oregon, USA, 2003.

[74] P. B. Wilson. A single wire has also been used for estimating transverse impedances of PEP components. Private communication.

[75] R. Wegner, W. Wuensch, G. Burt, B. Woolley, Bead-pull measurement method and tuning of a prototype CLIC CRAB cavity, LINAC2014, Geneva, Switzerland, 2014. 
[76] J. Shi, A. Grudiev, W. Wuensch. Tuning of X-band traveling-wave accelerating structures, Nuclear Instruments and Methods in Physics Research A 704 (2013) 14-18.

[77] H. Wang, J. Guo, Bead-pulling measurement principle and technique used for the SRF cavities at JLab, US Particle Accelerator School Lecture Series: "SRF practices and hands-on measurements", 2015.

[78] G. Jaume, RF Bead pull measurements of the DQW cavity, Summer Student Report, CERN, 2015.

[79] J. J. Garcia Garrigos, Development of the Beam Position Monitors for the Diagnostics of the Test Beam Line in the CTF3 at CERN, PhD thesis, 2013.

[80] ANSYS HFSS EM Field Simulator, http://www.ansys.com/Products/ Simulation+Technology/Electromagnetics/Signal+Integrity/ANSYS+ HFSS

[81] How many data points are enough?, http://www2 . phy.ilstu.edu/ wenning/ slh/How_Many_Data_Points.pdf

[82] Waveguide taper engineering using coordinate transformation technology, https: //arxiv.org/pdf/1001.1254.pdf, 2010.

[83] Measuring Setup Assembly, https://edms.cern.ch/ui/\#!master/search/ view? object type=document\&query=CLIASTNDQ159\&latestversion= false\&hideobsolete=true\&dadvanced=false\&global=true, 2016.

[84] Linear Stages manual, https://www.newport.com/medias/sys_master/ images/images/h2c/h45/8797115613214/ILS-User-Manual .pdf, 2015.

[85] Rotation Stage manual, https://www.newport.com/medias/sys_master/ images/images/h06/h0a/8797298229278/URS-B-User-Manual .pdf, 2013.

[86] ESP300 controller manual, http://ridl.cfd.rit.edu/products/manuals/ Newport \\%20ESP300/ESP300.pdf, 2002.

[87] Testo 174T - Mini temperature data logger, https://www.testo.com/en/ search?text $=174 \mathrm{~T}$

[88] UMR Precision, Double Row Ball Bearing Linear Stages M-UMR3.5, https:// www . newport. com/p/M-UMR3. 5

[89] Copper-Beryllium wire, http://www.goodfellow.com/catalogue/ GFCat4I.php?ewd_token=JQXbvaPLyARCsqQs1utccIpsFIoeKp\&n= JoKlqcifQ3] qwD2GOnLn7ZGdgHpdL8

[90] VNA ZVA24 manual, http://www.av.it.pt/Medidas/Data/Manuais $\backslash$ $\% 20 \& \backslash \% 20$ Tutoriais/40b $\ 20-\backslash \% 20 \mathrm{VNA} \backslash \% 20-\backslash \% 20 \mathrm{ZVB} 20 / \mathrm{CD} /$ documents / Product_Information/ZVA_Datasheet.pdf, 2010.

[91] LabVIEW, http://www.ni.com/en-gb/shop/labview.html

[92] WFM QC setup taper assy, https://edms.cern.ch/ui/\#! master/search/ view?object type=document\&query=CLIASTNDQ136\&latestversion= false\&hideobsolete=true\&dadvanced=false\&global=true, 2015 . 
[93] CMM Leitz Infinity, http://www.hexagonmi.com/products/ coordinate-measuring-machines/bridge-cmms/leitz-infinity

[94] ZEISS PRISMO CMM, http://www.msi-viking.com/assets/images/ downloads/productspecsheets/ZEISS $\backslash \% 2$ OSpec $\backslash \% 20$ Sheets/ZEISS_ PRISMO_CMM.pdf

[95] Technical Datasheet of the SMA Straight Female, Metal Through the Wall, 2 Hole Flange http://www.cmpter.com/UpLoadFile/Image/2013/0514/ 60-02-9E2-093.pdf

[96] Technical Datasheet of the R125.414.000 SMA, http://docs-europe. electrocomponents. com/webdocs/1422/0900766b81422b1f.pdf

[97] N. Galindo Munoz, N. Catalan Lasheras, S. Zorzetti, M. Wendt, A. Faus Golfe, V. Boria Esbert, Electromagnetic field pre-alignment of the Compact Linear Collider (CLIC) accelerating structure with help of wakefield monitor signals, IBIC2015, Melbourne, Australia, 2015.

[98] Active Optical Table, https://wwW.newport.com/p/M-VIS3048-PG2-325A, 2009.

[99] Hexapod HXP 100-MECA from Newport, https://www.newport.com/p/ HXP100-MECA, 2015.

[100] R\&S Oscilloscope RTO1024, https://www.rohde-schwarz.com/es/ producto/rto1000-pagina-de-inicio-producto_63493-191808.html

[101] N. Galindo Munoz, N. Catalan Lasheras, A. Grudiev, A. Faus Golfe, V. Boria Esbert, Pre-alignment of accelerating structures for compact acceleration and highgradient using in-situ radiofrequency methods. IPAC2016, Busan, Korea.

[102] N. Galindo Munoz, N. Catalan Lasheras, A. Faus Golfe, V. Boria Esbert. Prealignment techniques developments and measurement results of the electromagnetic center of warm high-gradient accelerating structures, IPAC2017, Copenhagen, Denmark, 2017.

[103] Micha Dehler, private communication. 